\title{
Social Aspects of Discussions AfFect Capitulation AND SUSCEPTIBILITY TO FALSE MEMORIES
}

\author{
by \\ Lauren Yseult French
}

\begin{abstract}
A thesis submitted to the
Victoria University of Wellington

in fulfillment of the requirements for the degree of

Doctor of Philosophy

in Psychology
\end{abstract}

Victoria University of Wellington

2008 


\begin{abstract}
People talk. People talk to entertain each other, to divulge news, and to gain support. Additionally, people talk about shared experiences to figure out what "really" happened. But does talking about the past change what we remember? That is the overarching question of the research presented in this thesis.

People remember the same events in different ways; consequently, when people discuss the past, they might come across new information. To examine how discussion affects people's memories, we must know what happened during a target event and must create conflicts in the discussion to see how those conflicts affect people's memories. To overcome these challenges, I used the MORI technique to present different viewers with different movies on the same screen at the same time (Mori, 2003; 2007). The MORI technique allows people to feel that they have shared an experience - they sit side-by-side and ostensibly watch the same movie-yet systematic differences are introduced into their memories, and the effect of those differences can be tracked through discussion.

I report a series of experiments that examine the efficacy of the MORI technique and investigate how different social factors contribute to false memories. Each experiment used a variation of the same basic three-stage procedure. First, pairs of people each unwittingly watched slightly different versions of an event. Next, pairs answered questions about the event together; some questions guided them to discuss details for which they had seen contradictory information. Finally, subjects completed a memory test individually to determine what each person really remembered about the event.

In short, when people watched a movie via the MORI technique, they could see and remember the details of the movie (Experiments $1 \mathrm{~A}$ and $1 \mathrm{~B}$ ), and they did not notice or implicitly remember details from the alternate (blocked) movie version-the version their partner saw (Experiments $3 \mathrm{~A}$ and ${ }_{3} \mathrm{~B}$ ). Additionally,
\end{abstract}


discussion corrupted people's memories (Experiments $2 \mathrm{~A},{ }_{2} \mathrm{~B}, 4,5$ and 6 ). People were influenced by their partner's suggestions: they falsely remembered details from their partner's version of the event, even though those details contradicted what they personally saw. Consistent with the Source Monitoring Framework, the corrupting influence of the discussion depended on social factors in the interaction (Johnson, Hashtroudi, \& Lindsay, 1993; Lindsay, 2008). For instance, people were more likely to remember false details that their romantic partner suggested than false details that a stranger suggested (Experiment 4). Additionally, leading people to believe that their counterpart's vision was better or worse than their own led them to be more or less influenced by their counterpart's false suggestions (Experiment 6).

In sum, when people share an experience and discuss it they can come to remember seeing things that they were only told about after the event. In other words, corroboration does not equal accuracy. I discuss the possiblebeneficial—mechanisms underlying these memory errors; draw parallels between my research and research on social influence, group remembering and transactive memory systems; discuss theoretical, methodological and practical implications, and suggest potential applications of my findings and avenues for future research. 


\section{Acknowledgments}

I'm not sure that I ever really believed I would get to this part. But here I am, and one thing is for certain-I couldn't have made it on my own. I have received endless help, encouragement and support over the past four years, and I'm not sure I can adequately express my gratitude in words. To everyone that has been there for me, I hope I can do you justice.

First, to Maryanne. Thank you for everything-your guidance, support, and encouragement, your open door. Thank you for inspiring me, challenging me and teaching me to think.

To Kaz, thank you for your generous support and for your encouragement of this research.

Thank you to the hundreds of people that have taken part in these studies; none of this research would have been possible without you.

Thank you to Victoria University for the scholarships that have supported me over the past four years.

Garry Labsters-past and present. Thank you for inspiring me, encouraging me and showing me it could be done. And especially to those of you that have done time with me-Deryn, Matt, Mel, Soph (O.F.F.), Eryn, and Jeff-you have been a fantastic group of people to work (and play) with. Thank you for your contributions to my research - whether ideas about design, figuring out formulas, making movies, posing for photos, challenging my explanations-you have all been amazing. Thanks also to Mike, Vicki, Rachel, Ken, and especially to Toni, for your contributions to this research.

To Chad, and everyone that helped make the Chad movie-the stars and those of you behind the scenes-thanks heaps!

To the staff in the School of Psychology. Thank you especially to the administrative and technical staff who have put up with my requests and questions for the past four years, and usually with a smile-you have made life much easier. Thanks also to all the academics that have taken time out their days to help me-every time I asked a question, people were only too happy to help figure out the answer, or at least help figure out the way to find the answer. Thank you to everyone in the School for making it such a nice place to be.

To the third floor gang of past and present: it has been a fun four yearsthank you for all the good times and the laughs.

My family and friends-especially Gran and Grandad, Mum and Warwick, Dad and Alma. Where would I be without you? I cannot possibly convey how much love and support you have given me over the past four years. Thank you for your support, encouragement and advice; thank you for having a listeningear every time I needed one, and thank you for believing in me.

And finally, to Dolan-my rock. Thank you for everything you have done to help me get here. Thank you for being the house-husband in the last few months and for taking good care of me; thank you for being interested-or at least "interested"-when I talked about my research, and thank you for your dutiful proofreading (BTW its $\$ 1$ per mistake for any that you missed). But seriously, thank you for your understanding and your patience; thank you for encouraging me, and most of all thank you for believing in me. You make a good rock. 


\section{Table of Contents}

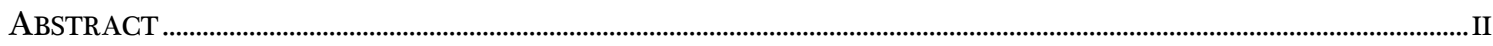

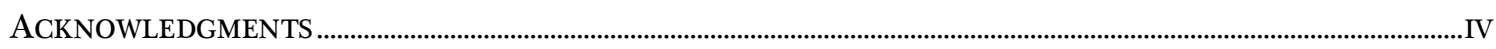

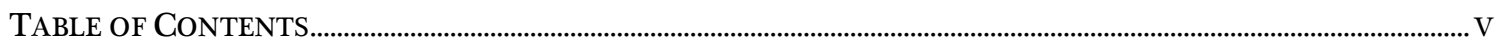

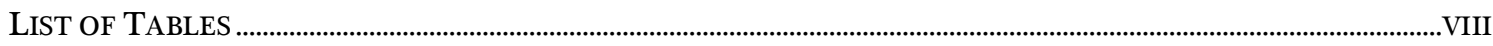

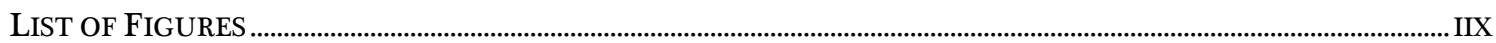

CHAPTER 1

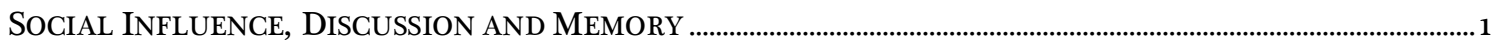

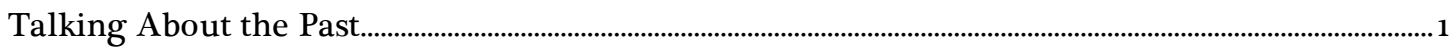

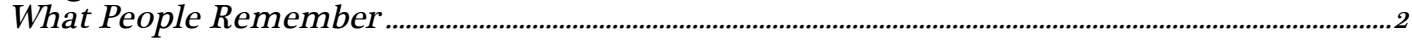

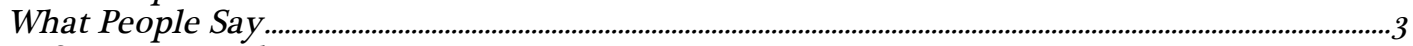

Misinformation and Memory .................................................................................................................................

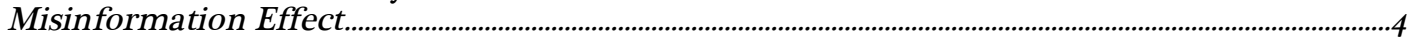

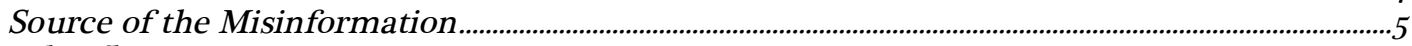

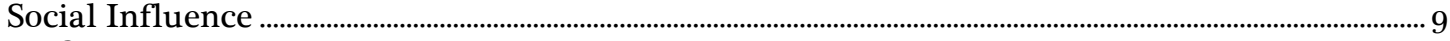

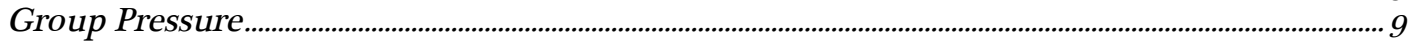

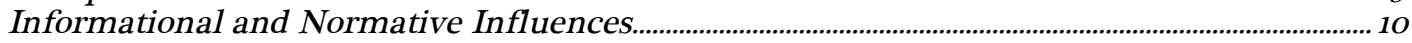

Transactive Memory Systems ................................................................................................................................. 10

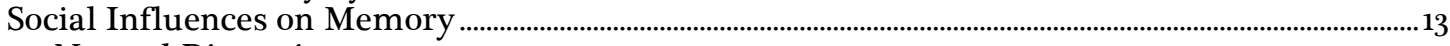

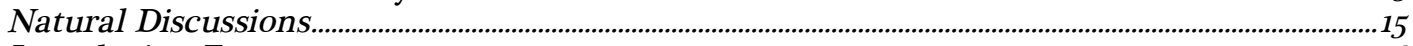

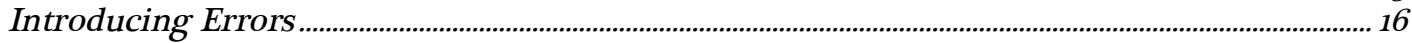

The MORI Technique ........................................................................................................................................... 19

Background to the MORI Technique ......................................................................................................... 19

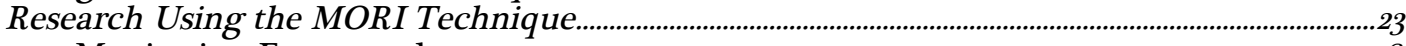

Source Monitoring Framework.....................................................................................................................26

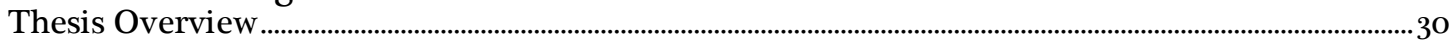

\section{CHAPTER 2}

EXPERIMENT 1: MISINFORMATION MATERIALS AND THE MORI TECHNIQUE..................................................... 34

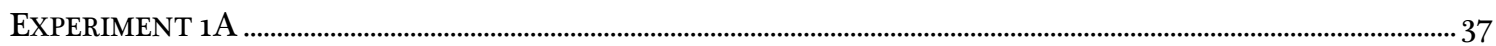

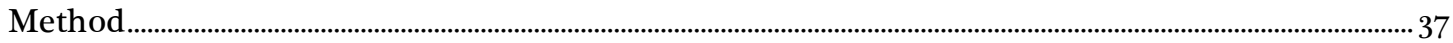

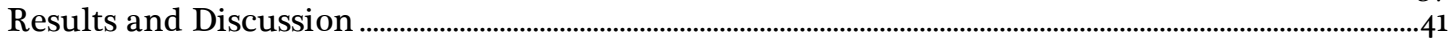

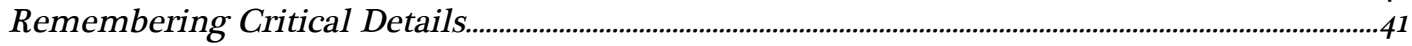

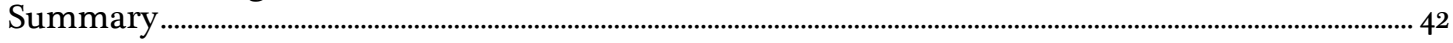

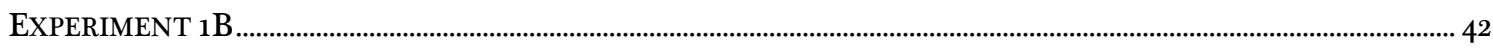

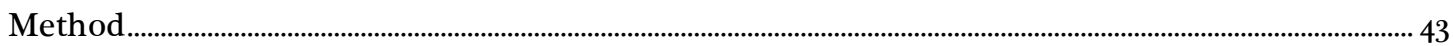

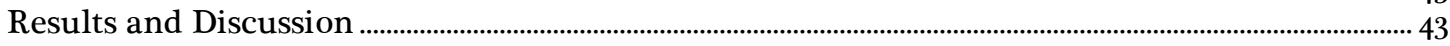

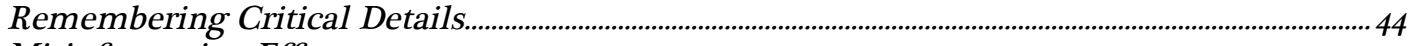

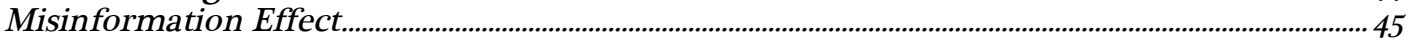

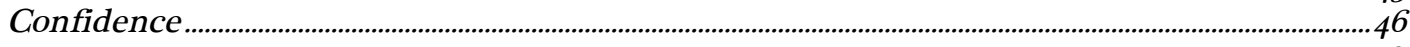

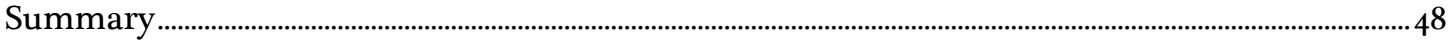

CHAPTER 3

EXPERIMENT 2: MISINFORMATION DELIVERED VIA DISCUSSION …….....................................................................49

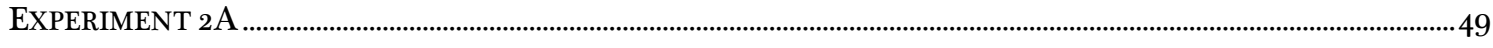

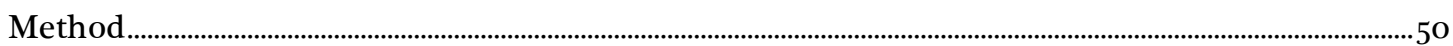

Results and Discussion .....................................................................................................................................52

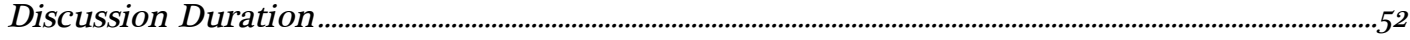

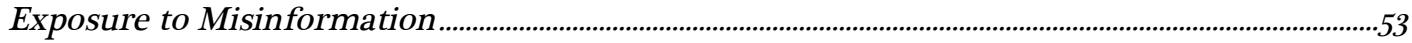

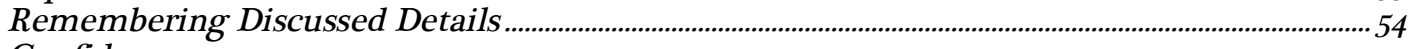

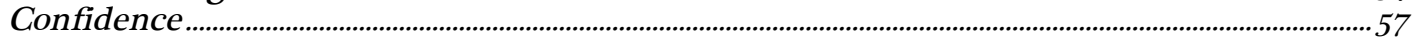

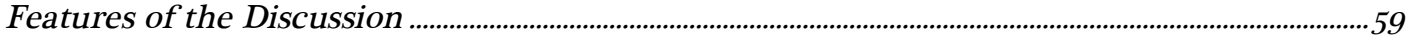

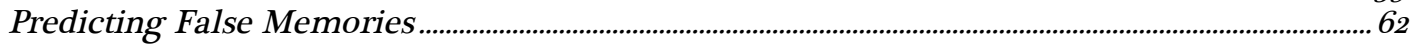

Summary. 


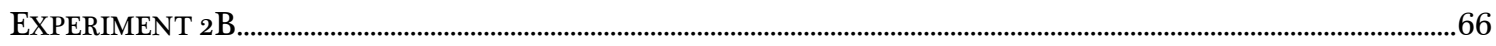

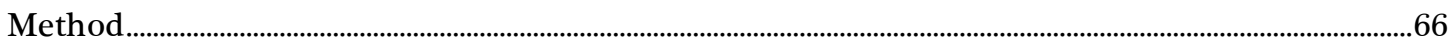

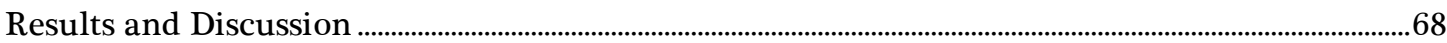

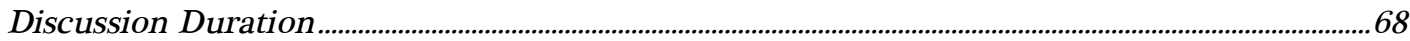

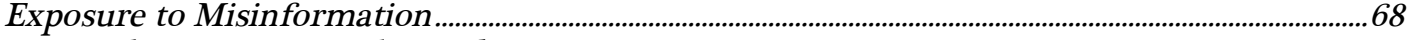

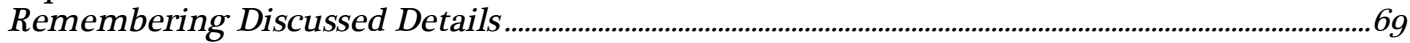

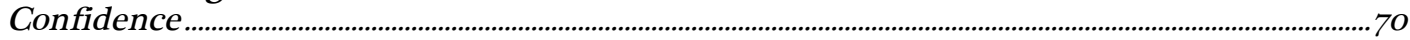

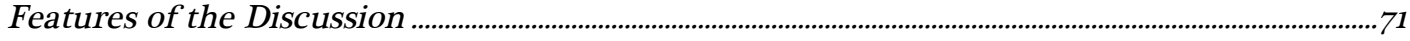

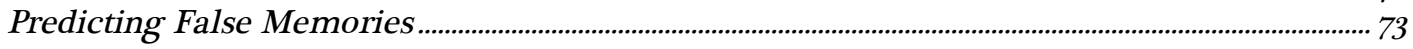

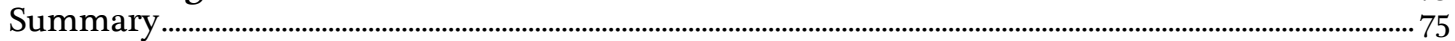

CHAPTER 4

EXPERIMENT 3: NotICING AND REMEMBERING THE ALTERNATE MOVIE ..............................................................77

EXPERIMENT 3A: EXPLICIT NOTICING ……….........................................................................................................79

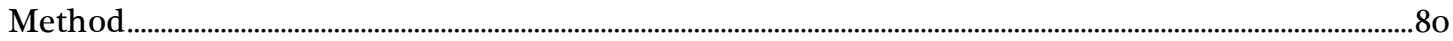

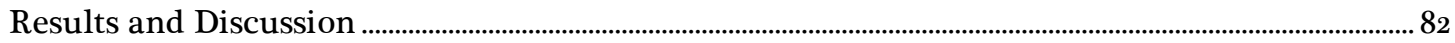

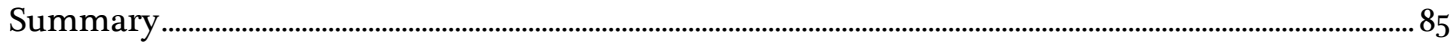

EXPERIMENT 3B: IMPLICIT MEMORY ………............................................................................................................86

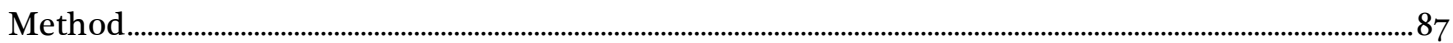

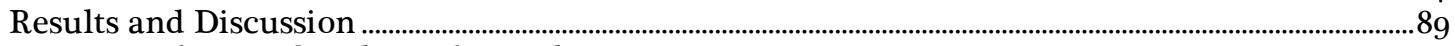

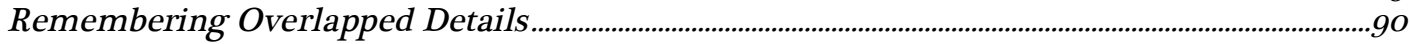

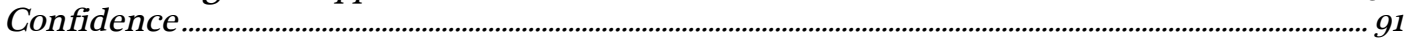

Remembering Details from the Alternate Movie Version ....................................................................92

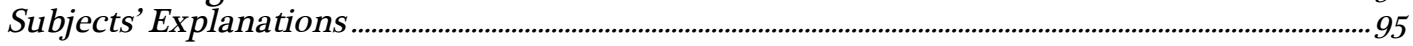

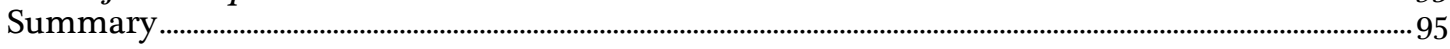

CHAPTER 5

EXPERIMENT 4: ROMANCING THE MISINFORMATION MESSENGER .......................................................................98

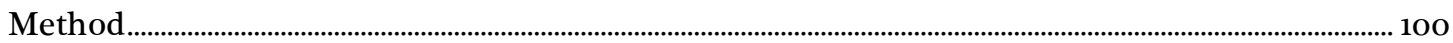

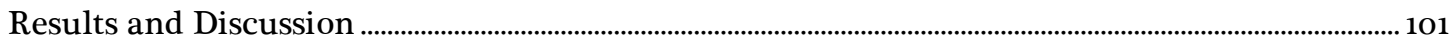

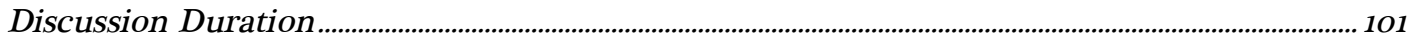

Exposure to Misinformation .......................................................................................................................... 102

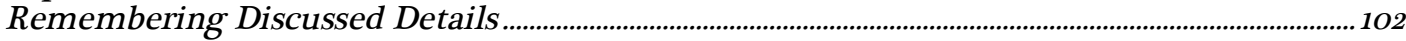

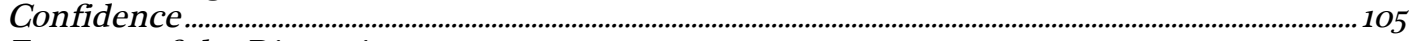

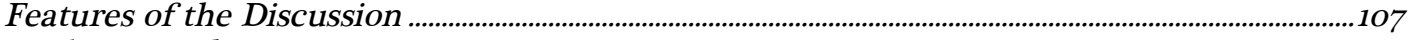

Predicting False Memories ...................................................................................................................

Summary ....................................................................................................................................................................112

CHAPTER 6

EXPERIMENT 5: GREAT AND NOT-SO-GREAT EXPECTATIONS........................................................................................116

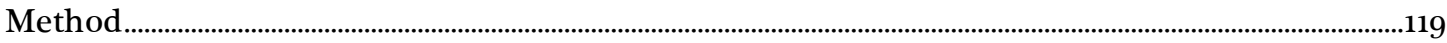

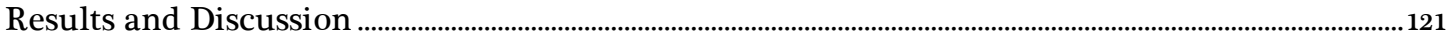

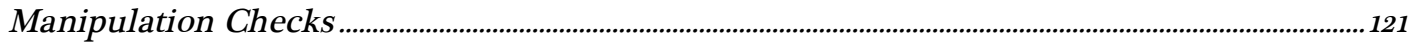

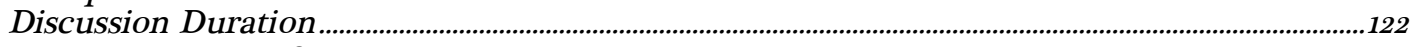

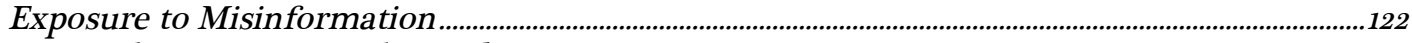

Remembering Discussed Details ..............................................................................................................122

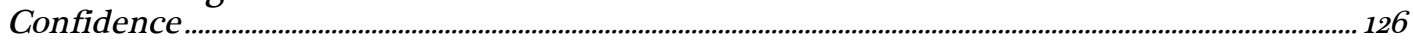

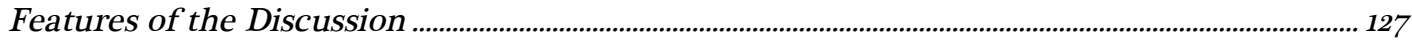

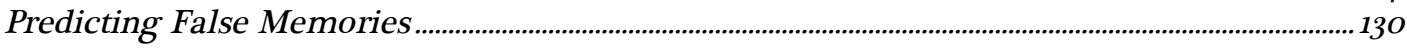

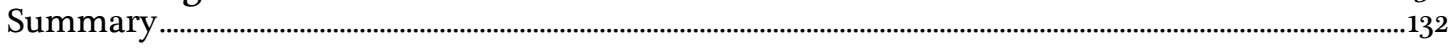

CHAPTER 7

EXPERIMENT 6: EXPecting the Best Protects People from FAlse MeMORIES that ARISE From

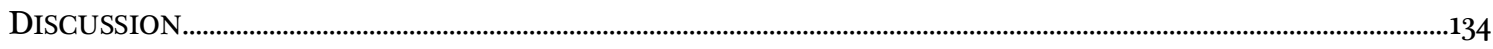

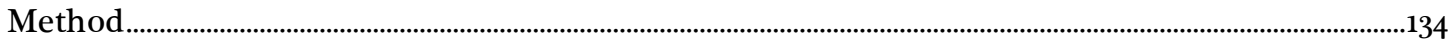

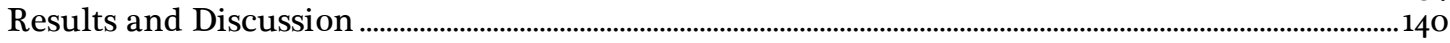

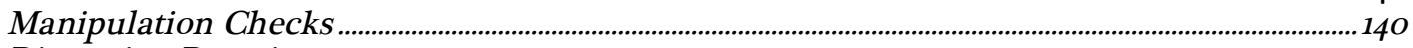

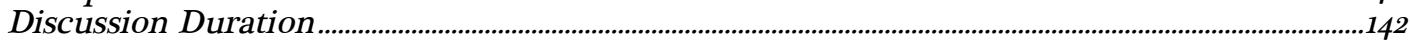

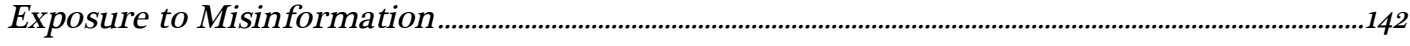


Remembering Discussed Details

Confidence.

152

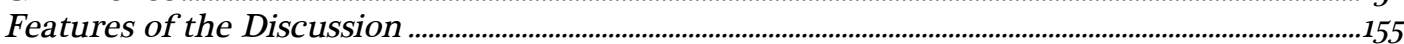

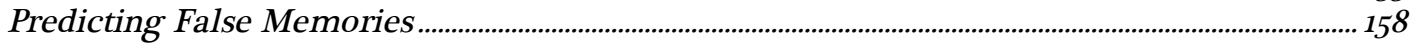

Summary

.160

\section{CHAPTER 8}

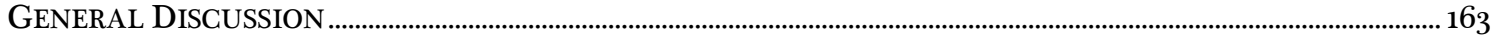

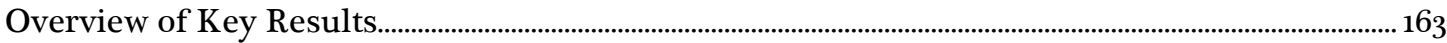

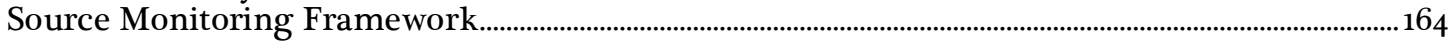

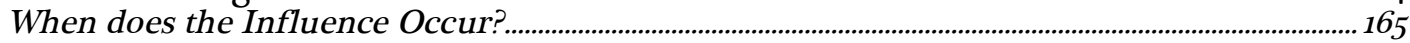

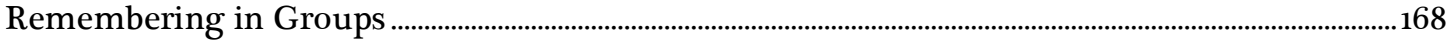

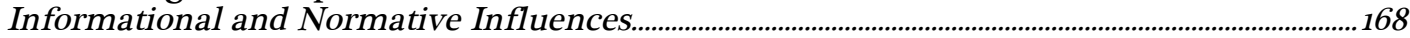

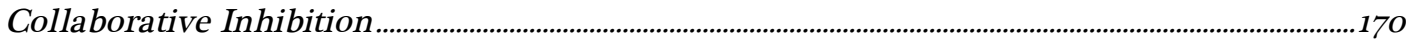

Expertise and Group Remembering ............................................................................................................171

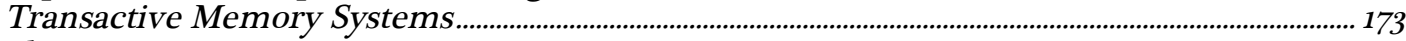

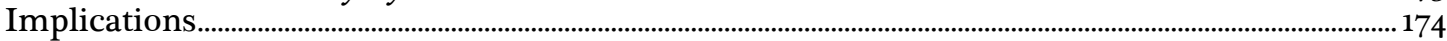

Source Monitoring Framework …………....................................................................................... 174

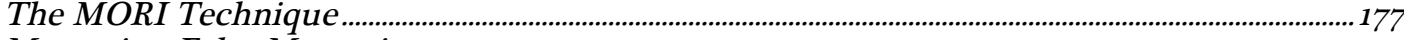

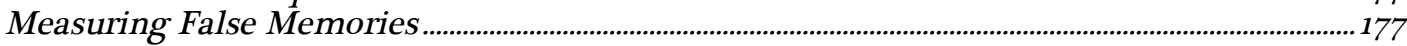

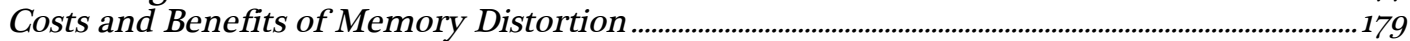

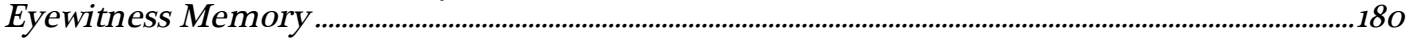

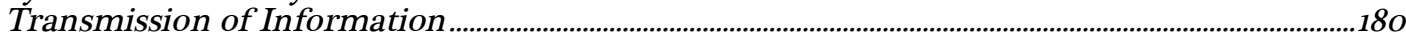

Remembering More Accurate Information. .........................................................................................181

Mind Over Memory Distortion ..........................................................................................................................181

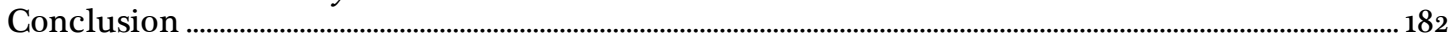

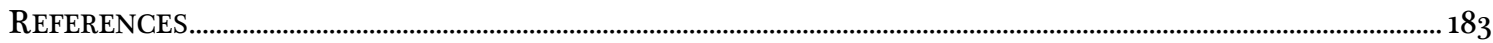

APPENDIX A: SAMPLE MISINFORMATION NARRATIVE............................................................................................... 200

APPENDIX B: EXAMPLE DISCUSSION QUESTIONS FOR ERIC MATERIALS.................................................................202

APPENDIX C: RECOGNITION TEST FOR ERIC MATERIALS .......................................................................................203

APPENDIX D: EXAMPLE DiSCUSSION QUESTIONS FOR CHAD MATERIALS ...........................................................208

APPENDIX E: RECOGNITION TEST FOR CHAD MATERIALS.................................................................................. 209

APPENDIX F: QUESTIONS IN RED HERRING TEST BOOKLET ......................................................................................214

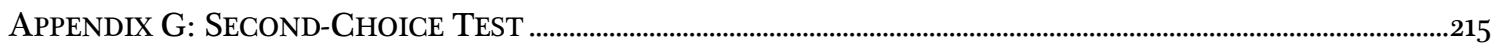

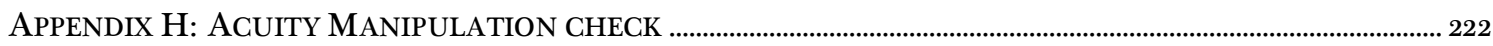

APPENDIX I: CREDIBILITY MANIPULATION CHECK ………................................................................................. 224

APPENDIX J: INDEPENDENT VS. DYADIC ANALYSES............................................................................................ 225

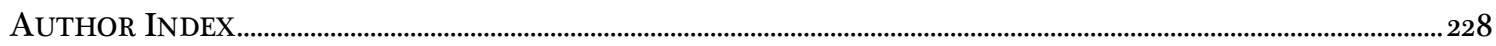

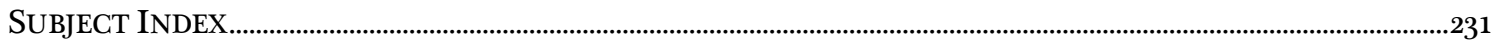




\section{List of Tables}

Table 2.1. Critical differences between Takarangi et al.'s (2006) movie versions ........................................ 36

Table 2.2. Updated critical details in revised versions of Takarangi et al.'s (2006) movie ..................... 43

Table 3.1. Proportion discussion feature occurrence and subsequent proportion subjects misled for 81 instances subjects exposed to misinformation....................................................................................................6o

Table 3.2. Critical differences between Chad movie versions............................................................................67

Table 3.3. Proportion discussion feature occurrence and subsequent proportion of subjects misled for 106 instances that subjects were exposed to misinformation ...................................................................... 73

Table 4.1. Critical details in each of the four half-length Chad movie versions.........................................8 88

Table 5.1. Proportion of strangers and couples who-when exposed to at least two misleading details-were never, sometimes, and always misled ..............................................................................................104

Table 5.2. Proportion discussion feature occurrence and subsequent proportion of subjects misled for 154 instances that subjects were exposed to misinformation......................................................................108

Table 6.1. Expectancy combinations based on subject-pairs' assigned glasses............................................120

Table 6.2. Proportion of subjects who-when exposed to at least two misleading details-were never, sometimes, and always misled, by expectancy condition........................................................................125

Table 6.3. Proportion discussion feature occurrence and subsequent proportion of subjects misled for 436 instances that subjects were exposed to misinformation..

Table 7.1. Proportion of subjects who-when exposed to at least two misleading details- were never, sometimes, and always misled, by expectancy condition.

Table 7.2. Proportion discussion feature occurrence and subsequent proportion of subjects misled for 253 instances that subjects were exposed to misinformation. 


\section{List of Figures}

Figure 1.1. Illustration of polarisation filters. 21

Figure 1.2. Illustration of polarising glasses worn to watch $3 \mathrm{D}$ movies. 21

Figure 1.3. Illustration of polarising glasses worn for MORI technique. . .22

Figure 1.4. Illustration of equipment set up for MORI technique 24

Figure 2.1. Photographs of boxes ostensibly containing different strengths of "Acuity Glasses" and people wearing polarisation glasses. ...38

Figure 2.2. Photographs of equipment used for MORI technique. 39

Figure 2.3. Proportion of correct control responses for each critical detail 42

Figure 2.4. Proportion of correct control responses for each critical detail 44

Figure 2.5. Proportion of correct responses for control and misled details. 45

Figure 2.6. Proportion of correct control and misled responses for each critical detail.. .46

Figure 2.7. Mean confidence for control and misled details .47

Figure 2.8. Mean confidence for correct responses on control details (true memories) and incorrect responses on misled details (false memories). .48

Figure 3.1. Example of slides used in discussion for critical questions and filler questions...................51

Figure 3.2. Proportion of correct responses for nondiscussed and discussed details. 55

Figure 3.3. Proportion of correct nondiscussed and discussed responses for each critical detail.......56

Figure 3.4. Mean confidence for nondiscussed and discussed details. .58

Figure 3.5. Mean confidence for correct responses on nondiscussed details (true memories) and incorrect responses on discussed details (false memories)

Figure 3.6. Proportion of correct responses for nondiscussed and discussed details

Figure 3.7. Proportion of correct nondiscussed and discussed responses for each critical detail......70

Figure 3.8. Mean confidence for nondiscussed and discussed details .$\cdot 71$

Figure 3.9. Mean confidence for correct responses on nondiscussed details (true memories) and incorrect responses on discussed details (false memories)

Figure 4.1. Overlapped laundry/bedroom critical scene photographed through no polarising filter, horizontal polarising filter, and vertical polarising filter.

Figure 4.2. Proportion of correct responses for nonoverlapped and overlapped details. . .90

Figure 4.3. Proportion of correct nonoverlapped and overlapped responses for each critical detail

Figure 4.4. Mean confidence for nonoverlapped and overlapped details. 92

Figure 4.5. Proportion of first and second choices correct for alternate movie version for nonoverlapped and overlapped details 
Figure 4.6. Proportion of first choices correct for alternate movie version for nonoverlapped and overlapped details

Figure 4.7. Proportion of second choices correct for alternate movie version for nonoverlapped and overlapped details

Figure 5.1. Proportion of correct responses for nondiscussed and discussed details by relationship

Figure 5.2. Proportion of correct nondiscussed and discussed responses for each critical detail....103

Figure 5.3. Mean confidence for nondiscussed and discussed details by relationship. . .106

Figure 5.4. Mean confidence for correct responses on nondiscussed details (true memories) and incorrect responses on discussed details (false memories) by relationship.....................................107

Figure 6.1. Proportion of correct responses for nondiscussed and discussed details by expectancy condition

Figure 6.2. Proportion of correct nondiscussed and discussed responses for each critical detail...124

Figure 6.3. Mean confidence for nondiscussed and discussed details by expectancy condition....... 126

Figure 6.4. Mean confidence for correct responses on nondiscussed details (true memories) and incorrect responses on discussed details (false memories) by expectancy condition................. 127

Figure 7.1. Updated box labels for different strengths of "Acuity Glasses.”...................................................135

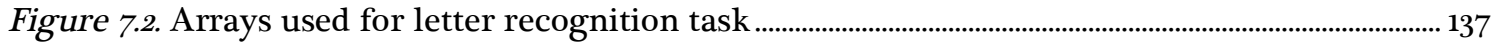

Figure 7.3. Celebrity photographs used for face recognition task ...................................................................... 138

Figure 7.4. Mean self-partner comparison ratings by expectancy condition .................................................141

Figure 7.5. Proportion of correct responses for nondiscussed and discussed details by expectancy condition

Figure 7.6. Proportion of correct nondiscussed and discussed responses for each critical detail...150

Figure 7.7. Mean confidence for nondiscussed and discussed details by expectancy condition.......153

Figure 7.8. Mean confidence for correct responses on nondiscussed details (true memories) and incorrect responses on discussed details (false memories) by expectancy condition................. 154 


\section{Chapter 1}

\section{Social Influence, Discussion and Memory}

Talking About the Past

We do not let go of one moment as soon as the next moment arrives; instead, we spend our lives constantly looking back and talking about the past. We listen as our colleagues, friends and families tell us about what they did or saw or heard and we respond by telling them what we did or saw or heard. Sharing stories is so important to us that when we cannot share them in person, we share them by post, on the phone, via text, email, web-cam, or even by blog.

People tell each other about their experiences for a wide variety of reasons: to entertain and humor each other; to share knowledge and opinions; to gain sympathy, attention, approval and trust; and to persuade each other of their views (Bartlett, 1932; Edwards \& Middleton, 1987; Marsh \& Tversky, 2004; Ross \& Conway, 1986; Weldon \& Bellinger, 1997). People also share stories of their past as a way to explain who they are, to define their identity, their background, relationships, motivations and goals (Barclay \& DeCooke, 1988; Edwards \& Middleton, 1986; Fivush, 1991; Fivush, Haden, \& Reese, 1996; MacDonald, Uesiliana, \& Hayne, 2000; Nelson, 2003). By explaining what they have done, people tell each other what they are like, and whether they would like each other (Reese \& Brown, 2000). As such, when people discuss the past, it provides a means to create relationships with others.

As well as telling others about the individual experiences they have had, people also talk about experiences they have shared. Reminiscing with others provides a means to investigate what "really" happened; discussion reminds people of details they had forgotten and allows them to negotiate and create a shared and verified account of the past (Edwards \& Middleton, 1986; Edwards \& Middleton, 1988; Hardin \& Higgins, 1996; Meade \& Roediger, 2002; Ross, 1997). 
Additionally, by talking about their past, people emphasise their shared history and the things they have in common, and as a result, they strengthen relationships with each other (Barclay, 1996; Fivush, et al., 1996; Halbwachs, 1980; Weldon \& Bellinger, 1997).

For the most part, accuracy is not a primary concern of autobiographical remembering (Hyman \& Faries, 1992, see also Hyman \& Loftus, 1998; Neisser, 1988; Neisser \& Harsch, 1992). However, for other types of memories—such as eyewitness memories-accuracy is paramount. The justice system relies on people to accurately remember what happened at a crime or accident; the course of the investigation, any charges laid, and the eventual outcome-whether conviction, incarceration, financial liability, or discharge-all depend in part, on the accuracy of the remembered information. Given how much people talk about the past, it is important to investigate at how discussion might influence what they remember.

\section{What People Remember}

One reason that discussion might affect people's memories is that different people remember different things about the same events. As a result, when people talk about the past together, they might come into contact with new information. What people notice and remember depends on personal factors such as their knowledge or beliefs, and situational factors such their perspective, involvement or attention; because these factors are necessarily different for different people, the end result is that different people remember different details (Bartlett, 1932; Cuc, Ozuru, Manier, \& Hirst, 2006; Edwards \& Middleton, 1986; French, Sutherland, \& Garry, 2006; Ikier, Tekcan, Gulgoz, \& Kuntay, 2003; Loftus, Ledivow, \& Duensing, 1992; Ross, 1997; Sheen, Kemp, \& Rubin, 2001).

In fact, research shows that when people recall the same event their memory reports are often vastly different. For example only a small portion (25\%) of the 
details sibling-pairs recalled about shared childhood events matched each other's recall (French et al., 2006). Similarly, families who independently recalled a story recalled vastly different details, with an overlap of only $4 \%$ between all four family members (Cuc et al., 2006).

Sometimes people remember events so differently that their memory reports contradict each other. For instance, the majority of the twins in a study by Sheen et al. (2001) reported at least one disputed memory; these twins each remembered the same event from their childhood, agreed that the event happened to only one of them, but did not agree on who it actually did happen to (see also Ikier et al., 2003). In short, given the different ways that people remember the same events, when people discuss the past it is very likely that they will be exposed to new information.

\section{What People Say}

Another reason that people might be exposed to new information about the past during discussion is that what people say doesn't necessarily match what they remember. The way people talk about their past depends on their goals, the social context, the audience, and their ability to describe their memory (Bartlett, 1932; Echterhoff, Higgins, \& Groll, 2005; Edwards \& Middleton, 1986; Marsh, 2007; Marsh \& Tversky, 2004; Ross, 1997). For example, people might talk about the past to entertain their friends, to provide information, or to gain sympathy.

Marsh and Tversky (2004) found that when people talked about past experiences, sometimes they minimised and excluded details, and other times they exaggerated and added details. Additionally, when the same people told the same stories repeatedly, they retold them in different ways for different audiences. Interestingly, these people were aware of the distortions in their accounts, but they did not always consider their stories to be inaccurate just because they were distorted. 
In sum, remembering the past, describing experiences to each other and reminiscing together are all common activities. However, because people undoubtedly experience things differently, remember different things about the same events, and talk about the past in a way that does not always reflect what they remember, when people discuss the past, they might be exposed to new information. How does this new information affect people's memories?

$$
\text { Misinformation and Memory }
$$

\section{Misinformation Effect}

In fact, a whole body of research on the misinformation effect shows that when people are exposed to new information about an event, it can change what they remember about it (for example see Belli, 1989; Lindsay, 1990; Loftus, 1991; Loftus, Miller \& Burns, 1978; Loftus \& Palmer, 1974; Loftus \& Zanni, 1975; McCloskey \& Zaragoza, 1985). In misinformation effect studies, people typically watch a slide sequence or video of an event, such as a crime or accident. After watching the event, subjects are provided with some misleading information about it. This misinformation is usually embedded in leading questions that subjects answer, or in a written description of the event that subjects read or listen to. For instance, subjects might watch someone trying on a blue hat but later read that he tried on a black hat (Takarangi, Parker, \& Garry, 2006).

Later, subjects complete a surprise memory test to examine their memory of the original event. The typical result is that people incorrectly report seeing the misleading details that were only presented to them after the event. In these studies, people have come to remember completely false details, such as nonexistent buildings; they have misremembered the colour of different objects, for example remembering a blue hat as black, or a yellow cup as white; they have misremembered brands of certain products, for example remembering a can of Coke as a can of Pepsi, or a Time magazine as a Newsweek magazine; and they 
have misremembered what an object actually was, for example remembering a hammer as a screwdriver, or a yield sign as a stop sign (see for example Loftus, 1975; Loftus et al., 1978; McCloskey \& Zaragoza, 1985; Takarangi et al., 2006).

In sum, misinformation effect research shows that accessing new information after an event can change what people report about the event. Perhaps the effect is part of a broader mechanism that provides a means for people to absorb information from different sources and boost their knowledge and understanding of what happened—at a later date they know and remember much more about an experience than immediately afterward (Meade \& Roediger, 2002). Misinformation research shows that this process also creates the potential to contaminate people's memories when they are exposed to inaccurate information. However, some features of misinformation experiments might work to exacerbate the influence of misleading information. Specifically, the way that misinformation is conveyed in misinformation effect studies is quite different to how it might be conveyed in real life. Perhaps people would not be as influenced by misinformation encountered in the real world.

\section{Source of the Misinformation}

In most misinformation studies, the misleading suggestions come from the experimenter. One problem with this approach is that the experimenter knows the details of the target event very well. Thus, subjects might perceive the person providing the misinformation-the "misinformation messenger"- to be expert about the event, perhaps inflating the impact of their misleading suggestions (Smith \& Ellsworth, 1987; Vornik, Sharman \& Garry, 2003). Put another way, the same suggestions might have less impact if conveyed by someone less expert.

In fact, there is some evidence that people's perceptions of the misinformation messenger are crucial to the persuasiveness of the misinformation. One way that researchers have attempted to gauge the role of the misinformation messenger 
has been to tell subjects where the event description came from. For example, Dodd and Bradshaw (1980) showed subjects a slide sequence of a car accident, and then asked them to read a description of the event. For two groups, this description contained misinformation; these subjects were told that the description was provided to the police either by an innocent bystander or by the faulty driver. A third control group read a description with no misinformation. Subjects who were told the misleading description came from an innocent bystander showed the typical misinformation effect, reporting information that they read but never actually saw. Subjects who were told that the misleading description came from the faulty driver were no more misled than subjects who saw the control description. In other words, these subjects were able to resist the influence of the misinformation, and most likely they did so because the messenger had a good motivation to be dishonest.

A number of studies since Dodd and Bradshaw (1980) have produced similar results (for example see Ceci, Ross, \& Toglia, 1987; Echterhoff, Hirst, \& Hussy, 2005; Smith \& Ellsworth, 1987; Vornik et al., 2003). For instance, Echterhoff et al. found that subjects could resist the influence of misinformation when they were told that the messenger was untrustworthy or incompetent.

Another way that researchers have focused on the social aspects of misinformation is by showing subjects fake responses made by other hypothetical research participants (for example see Basden, Reysen, \& Basden, 2002; Betz, Skowronski, \& Ostrom, 1996; Highhouse \& Bottrill, 1995; Hoffman, Granhag, Kwong See, \& Loftus, 20o1; Itsukushima, Nishi, Maruyama, \& Takahashi, 2006; Luus \& Wells, 1994; Reysen, 2005; Walther, Bless, Strack, Rackstraw, Wagner, \& Werth, 2002). Like Dodd and Bradshaw (1980), Hoffman et al. (2001) compared the influence of different misinformation messengers; before making their own memory judgments, subjects saw a response that they were 
told was made by a graduate student participating in the study (high credibility) or randomly generated by a computer (low credibility). The results fit with those of Dodd and Bradshaw: when subjects were told that the answer came from the computer, it had no impact on their responses. Yet, when subjects were told that the answer came from a graduate student, their responses changed in line with the fake answer, increasing or decreasing subjects' accuracy depending on the accuracy of the fake response. In other words, subjects made judgments about who provided the information and those judgments determined the outcome for their memory reports.

In short, these studies show that people's judgments about the misinformation messenger are important. Yet social influences on memory may be much more complicated than simply deciding if the misinformation messenger is good or bad; in real life people are exposed to a whole host of factors beyond simply knowing who they are talking to. For example, when people are exposed to information during a discussion they might evaluate the speaker's goals and intentions (Wyer \& Gruenfeld, 1995). Furthermore, people may make social judgments based on factors such as the speaker's appearance, age, body language, gender, tone, confidence, as well as based on their own personal knowledge, beliefs and expectations about the speaker.

In fact, some research shows that the misinformation messenger does not need to be explicitly labeled as good or bad, but even very subtle factors conveyed via sociolinguistic cues can influence subsequent memory performance (Vornik et al., 2003). Vornik et al. presented subjects with misinformation embedded in an audio description read in either a North American or a New Zealand accent. Although accent alone did not determine the influence of the misinformation, subjects' perceptions of the speaker's power and social attractiveness did affect their susceptibility to the misinformation. 
Given the vast number of cues that can be conveyed in a real life conversation (for example sociolinguistic cues, body language), this finding provides support for the idea that misinformation maybe received very differently in real life interactions than in the misinformation studies described so far.

There is some evidence that people might use social cues-that are not available in a written narrative-to make judgments about the misinformation messenger and to assess the value of the misinformation. In a recent study, Itsukushima et al. (2006) provided misinformation by showing subjects either a video or a written transcript of someone who had supposedly already taken part in the study. Subjects were influenced by the misinformation in both conditions; however, this influence was stronger when subjects received the written misinformation. Although the situation of watching a video is still far removed from a real social interaction, one possible explanation for the difference is that subjects were able to make more detailed social judgments about the information presented on the video by judging factors such as the speaker's appearance, age, body language, linguistic skills, confidence; perhaps these judgments led subjects to be less influenced by the same misinformation.

Taken as a whole, these studies demonstrate that people's perceptions of the misinformation messenger play a role in determining the outcome for memory and that even subtle social cues can affect the influence of the misinformation. In short, misinformation received in a social interaction may be experienced very differently to misinformation received in a misinformation study.

Other research illustrates that making decisions and remembering in a social context is very different to doing so alone. Findings from social influence research and transactive memory research provide evidence that the features of real life social interactions may play an important role in people's decisionmaking, their behaviour and even what they remember. 
Social Influence

\section{Group Pressure}

Asch's classic studies $(1952 ; 1953 ; 1966)$ show that people's judgments and decisions can be extremely influenced by the presence and pressure of other people. Asch investigated the factors that led to social conformity; in other words, he examined the factors that would lead people to follow the crowd, or to refuse to follow the crowd.

Asch $(1952 ; 1953 ; 1966)$ asked groups of subjects—made up of one real subject and a number of confederates - to do a simple line judgment task; the goal was to choose the line from a group of alternatives that was the best match to another target line. On some trials the confederates selected the wrong line; as a result, subjects were in a situation where their perceptions were contradicted by the rest of the group. Subjects who completed the line judgment task independently made virtually no errors, yet subjects who completed the line judgment task in the group situation conformed to the majority-or at least toward the line length of the majority-in one third of all judgments. Whether subjects followed the crowd depended on the social dynamics of the situation, and specifically how many confederates there were, whether the confederates' judgments were unanimous, and the number of dissenters there were.

Interestingly, some subjects nearly always conformed to the group judgment, yet others never did; these results suggest that some kind of personality disposition might lead people to be more or less susceptible to social pressure. Asch suggested that a person's underlying character and self-confidence might determine whether that person will succumb to social pressure.

Since Asch's (1952; 1953; 1966) pioneering studies, many researchers have used variations of his method, conducting similar studies in different countries. Personality factors, culture, relationships among group members, and the 
specific characteristics of the situation all play a role in determining the impact of social pressure (for a review of these studies see Bond \& Smith, 1996). Informational and Normative Influences

In these social influence studies subjects might have gone along with the majority judgment for one of two reasons. Either, they believed that the incorrect judgment was actually correct or they copied the majority judgment even though they believed it was wrong (Deutsch \& Gerard, 1955). Deustch and Gerard named these two pressures informational influence (when someone believes that the majority judgment is accurate and uses it to inform their own judgment) and normative influence (when someone capitulates to the social pressure of the situation to be polite or save face). In fact, Deutsch and Gerard showed that both normative and informational influences contribute to the effects found in these types of studies: when subjects gave private judgments (rather than public) and there was no normative pressure to conform, they still went with the majority judgment some of the time. People use each other to verify reality-in this situation when other people contradicted subjects' perceptions, subjects sometimes took that contradiction to mean that the other people's judgments were more informative and credible than their own personal judgments (Deutsch \& Gerard, 1955).

These studies provide evidence that people succumb to pressures in social contexts that they would not encounter when doing the same tasks independently: people may bend their perceptions, decisions and judgments to fit with others. Other research shows that the social context has important implications for memory performance as well as decision making tasks.

\section{Transactive Memory Systems}

Most of the time, people know the people they talk to and know what those people know about. In close relationships, people know so much about each 
other that they are able to rely on each other as a kind of external memory aid, to boost their collective memory performance. For example, I know my partner is good at remembering directions but terrible at remembering phone numbers. On the other hand, he knows that I am good at remembering phone numbers, but terrible at remembering directions. Because we know each other's areas of expertise (and non-expertise), we can use each other in the same way that we use diaries, alarms and other memory aids to remember things that we wouldn't remember on our own (Stephenson, Kniveton, \& Wagner, 1991; Wegner, 1987; Wegner, Giuliano, \& Hertel, 1985). I know that I can easily get directions to a particular place by asking my partner where to go; equally, he knows if he needs a phone number he can ask me.

Wegner and colleagues used the term "transactive memory systems" to describe the way that people in relationships use each other's memories as extensions of their own (Wegner, 1987; Wegner et al., 1985). Transactive memory systems develop when people share responsibilities and learn about each other's expertise; in short, people might share transactive memory systems within intimate relationships, family groups, friendships and even work groups (Hollingshead, 1998a; Hollingshead, 200o; Wegner, 1987; Wegner et al., 1985).

Group memory systems work well to the extent that people in the group know each other and each other's expertise (Wegner, 1987). Expertise can be established in a number of ways. For instance, people in close relationships may gradually learn more about each other and each other's expertise as their relationship progresses. People who do not share close relationships but work on some task or project together might explicitly tell each other what they are good at. Alternatively people might rely on stereotypes of gender roles or occupation, or make inferences based on factors such as appearance or intelligence to decide what other people are good at, and thus whether the information conveyed is 
reliable (Hollingshead \& Fraidin, 2003; Wegner, 1987; Wegner, Erber \& Raymond, 1991; Wegner et al., 1985).

A number of studies have demonstrated that people in intimate relationships benefit from transactive memory systems (Hollingshead 1998a; 1998b; Wegner et al., 1991). For example, Wegner et al. (1991) recruited couples who had been together for at least three months, and found that they used each other as extensions of their own memories, outperforming pairs of strangers at remembering category exemplars in different areas of expertise (for example science, food, spelling). Similarly, Hollingshead (1998a, 1998b) showed that couples who worked together outperformed pairs of strangers who worked together at both general knowledge tests and remembering word lists. One important component in transactive memory systems is non-verbal communication. Hollingshead (1998b) found that intimate couples lost the benefit of their transactive memory when they communicated via computer rather than face to face, providing more evidence that the social dynamics of an interaction may be crucial to the outcome.

In sum, transactive memory systems enable people who know each other to store and access more information than they would alone. Members have specific areas of expertise to concentrate their cognitive effort on, and they know what others' areas of expertise are so they can access information from those areas. Additionally, because people know about each other's expertise (and nonexpertise) they can make judgments about the reliability and value of any information conveyed based on who mentioned it. However, transactive memory systems also create the potential for errors to creep into memory reports: transactive memory may be even more susceptible to distortion than individual memories are. In transactive memory systems, people rely on each other to remember information; because-by definition-these memories are external, it 
may be especially difficult for members to notice if the remembered information is replaced with other new or false information (Wegner et al., 1985; Wegner, 1987). As a result, if people know and trust their discussion partner, it may increase their risk of memory distortion; when people access information from a trusted person, they probably trust that the information is accurate and reliable.

Taken together, these two bodies of research show that factors in a social interaction can affect people's decisions, behaviours and memories. Not only are there a myriad of additional cues available during social interactions, but people's preexisting knowledge and beliefs about their discussion partners may have important consequences for decisions and memory. Although this research illustrates the potential for memory errors to come about through social pressure and transactive memory, it does not provide any evidence that memory is actually compromised through these processes.

\section{Social Influences on Memory}

Some real world examples illustrate how memory might be shaped through discussion. Take the case of Anna Lindh, the Swedish Foreign Minister who was stabbed in a shopping mall in 2003; she died the following day (Castle, 2003; Cowell, 2003a; 2003b; "Swedish Minister," 2003). According to Granhag, Ask and Rebelius (2005) the shoppers who witnessed the attack were taken to a room to wait for the police; in the meantime they talked about what they had seen. When the police did arrive, each witness was questioned in front of the others. Some of the witnesses' reports were extremely consistent, to the extent that some even used the very same adjectives to describe the attacker. Although it is possible that the witnesses might have used the same adjectives by chance alone, another possibility is that the discussion shaped their subsequent memory reports. Another case example illustrates how an error might occur. 
called Elliot's Body Shop. Several days later he set off a bomb to destroy the Murrah Federal Building in Oklahoma, killing 168 people and injuring another 850 (Thomas, 1997a). Three staff members from Elliot's Body Shop remembered that two men picked up the rental truck: McVeigh and an accomplice. One of the staff members provided a detailed description of the accomplice, which was widely publicised during the investigation. However, the description was eventually discredited, and the witness no longer knows whether McVeigh picked up the truck alone or with an accomplice (Thomas, 1997b; 1997c; 1997d). Interestingly, according to Memon and Wright (1999) the other two staff members—who both reported an accomplice-initially reported that only one man picked up the vehicle. Perhaps discussion between the staff members cued them to recall the previously forgotten second man; alternatively perhaps there was no accomplice, but one person made a mistake and through discussion, the other staff members came to remember someone that was not there (Skagerberg \& Wright, 2008; Gabbert, Memon \& Allan, 2003; Wright, Self \& Justice, 2000). Adults are not the only people to discuss their experiences; children might also come to remember things that they talk about together. Take for instance the Christchurch Civic Crèche Case (see Hood, 2001 for a thorough examination of the case). On the basis of the children's testimony, Peter Ellis-a childcare worker at the crèche-was accused and convicted of sexually abusing some of the children in his care. However, when suspicion was first raised by one of the crèche parents, no child had disclosed any abuse. In fact, it was not for several months that the children did start reporting abuse. During that time, many children still attended the crèche and were in regular contact with one another. One possibility is that the children-many of whom were being questioned by their parents and by interviewers from the Department of Social Welfare-were discussing these experiences with each other, and leading one another to 
remember things that had not happened (see Principe \& Ceci, 2002).

In all of these high profile cases, the witnesses' memory reports guided the course of the police investigation. Eyewitness' memory errors have the potential to mislead the investigation and waste valuable police time and resources. Indeed, if McVeigh really did go to Elliot's Body Shop alone, the massive manhunt to find his accomplice was nothing more than a wild goose chase.

Why did the people in these cases discuss their memories? Paterson and Kemp's (2006a) survey of witnesses suggests one important reason might be that discussion works to fill in the blanks. When people find out what other people know, and add that information to what they know, they have a better idea about what happened, and have more information to share with other people later on. It seems that — at least in principle—sharing memories with other people might work to improve memory performance by boosting the amount that people know. But perhaps discussion can also lead people to remember someone or something that was not there.

\section{Natural Discussions}

A number of studies have examined what happens to people's memories when they discuss a crime or serious event (for example see Alper, Buckhout, Chern, Harwood \& Slomovitz, 1976; Hollin \& Clifford, 1983; Underwood \& Milton, 1993; Warnick \& Sanders, 1980; Yarmey 1992; Yarmey \& Morris, 1998). In these studies subjects typically watch a video or listen to a recording of an event and then discuss it together. The results vary widely, with some studies showing that groups are more accurate at remembering, and others showing that groups are less accurate. One difficulty in drawing conclusions from these studies is that they all use different comparison groups. For instance, some studies compared memory reports given during a group discussion with reports given individually prior to discussion; other studies compared individual reports made by people 
who discussed the event with individual reports made by people who did not discuss the event. Finally, some of these studies required people to agree on their answers-perhaps these people reported details they did not remember simply because they were required to agree.

A more recent approach to research discussion and memory has been to compare the details that individuals recall pre and post discussion. For instance, Cuc et al. (2006) asked family members to read stories. Each person recalled the story individually, discussed it with the family, and then recalled it individually a second time. Family members adopted details from each other's reports into their own post discussion recall. Similarly, pairs of siblings who recalled shared childhood experiences individually and then in a discussion incorporated parts of each other's recall into their own reports (French et al., 2006).

Although these studies show that discussion may lead people to incorporate information from other's recall into their own, they do not tell us whether that information is accurate. It might be that group members simply reminded each other of forgotten details, boosting recall. Alternatively, rather than being cued to recall forgotten information, people may simply add new details to their reports, regardless of whether they previously noticed or remembered those details, and regardless of whether those details are accurate. One way to address the issue of accuracy is to introduce controlled errors into the discussion; errors provide a way to measure what happens when people are exposed to new information during a discussion, while ruling out the possibility that they are being cued to remember previously forgotten details.

\section{Introducing Errors}

The two main approaches researchers have used to introduce controlled errors into discussion have been to recruit confederates to plant false information, and to show two people slightly different materials with the idea that they will then 
discuss the differences and expose each other to new information.

Confederates. Confederates introduce controlled errors into a discussion in a systematic way, so that each subject is exposed to the same false information in the same way. In studies using this method, typically a subject and a confederate-who pretends to be another subject—view an event (or some materials) together. Afterwards, they discuss what they can remember; during this conversation the confederate mentions some false details. In some studies subjects give their memory responses during the discussion, and in other studies they complete an individual memory test following the discussion. The general result is that people come to remember information that was only mentioned by the confederate. For example, on the basis of confederate responses, people have come to remember seeing missing household items, non-presented words, non presented photographs, details missing from a movie and they have even come to remember performing actions that they did not do (Gabbert, Memon, Allan, \& Wright, 2004; Kassin \& Kiechel, 1996; Meade \& Roediger, 2002; Paterson \& Kemp, 2006b; Roediger, Meade, \& Bergman, 2001; Schneider \& Watkins, 1996; Shaw, Garven, \& Wood, 1997; Wright, Mathews, \& Skagerberg, 2005).

As well as leading people to remember less accurately, confederates can also lead people to remember more accurately. Specifically, when a confederate mentions some correct information during a discussion, people are more likely to give the correct response for that item too (for example, Paterson \& Kemp, 2006b; Schneider \& Watkins, 1996; Shaw, et al., 1997). Thus it appears that a helpful mechanism turns bad when people are exposed to bad information. These studies show what might happen to memory when people mention false information in a conversation. However confederates may behave fundamentally differently from people in natural discussions. Confederates are told not only to convey the misleading information confidently, they are also 
told not to comment on anything that the subject says during discussion (for example see Paterson \& Kemp, 2006b). This kind of dialogue may break normal conversational rules, constrain any social dynamics or sociolinguistic factors that contribute to memory conformity and may simply feel strange to the subject. Indeed, when subjects in a pilot study were paired with a confederate rather than with another subject they reported their discussions to be "stilted and awkward" (Cuc et al., 2006, p. 754).

Non-matching materials. To overcome the problem of confederates' nonnatural conversational behaviour, other researchers have asked pairs of subjects to have conversations together. To introduce errors into these more natural conversations, the experimenters show subjects slightly different versions of the target event (or materials) but lead them to believe they have seen the same thing. For example, Gabbert et al. (2003) filmed a crime event from two different angles; members of subject-pairs watched different versions of the movie. Importantly, there were two critical actions that were unique to each version of the movie-thus each subject in a pair saw two things that the other did not see. When the pairs discussed the event together they mentioned the unique details and exposed each other to misinformation; subjects later reported details that their partner only told them about, from the other version of the movie.

Using this method people have come to remember people and things that they had not seen, such as a non-existent criminal accomplice; they have misremembered details from stories and from pictorial scenes, for example, confusing a house with a tree, or a man with a woman; and they have incorrectly remembered a target character's actions, for example misremembering someone stealing cash from a wallet instead of stealing a credit card or instead of not stealing anything at all (Cuc et al., 20o6; Gabbert, Memon, \& Wright, 2006; Gabbert, Memon, \& Wright, 2007; Wright et al., 2005; Wright et al., 2000). 
Additionally, using a similar method, children have come to remember participating in events that they only heard about from their classmates (Principe \& Ceci, 2002).

This method overcomes the major shortcoming of research using confederates: two equal parties take part in a natural conversation together, without any predetermined scripting on one side. However, this method is not without its own shortcomings. Specifically, in order for subjects to have different experiences they are separated (either in space or in time) while viewing the critical event or materials. Later, subjects are brought back together to discuss what they have been led to believe was the same thing. For example, Gabbert et al. (2003) asked each subject to complete a filler task while the other watched the critical crime movie. However, the subjective experience of sharing an event with someone is not captured as well by experiencing the same event (or what people believe is the same event) as another person while being separated from that person as it is by genuinely experiencing an event together.

The MORI Technique

In an effort to capture the unique situation of sharing an experience, while still introducing systematic discrepancies into subjects' memory reports, Mori and colleagues developed a new presentation technique by adapting the light polarisation method used to present three-dimensional $(3 \mathrm{D})$ movies (Kanematsu, Mori, \& Mori, 2003; Mori 2003; Mori 2007). The MORI technique allows two viewers to see different movies on the same screen simultaneously-thus the viewers have the shared experience of watching the event together, but have systematic discrepancies in their memories, which can be brought into a discussion.

Background to the MORI Technique

Light Polarisation. 3D movies are created using the basic principles of light 
polarisation. Light waves travel in all directions; polarising filter works to block light waves of a certain direction, while allowing the waves of another direction to pass through (Hewitt, 2001). Put another way, polarised light waves travel in only one direction. For instance, when a polarising filter is aligned horizontally, only horizontal light waves pass through while vertical light waves are blocked; when a polarising filter is aligned vertically, only vertical light waves pass through while horizontal light waves are blocked.

When two polarising filters are placed in succession, only light waves of the same direction can pass through both (Hewitt, 2001; see Figure 1.1). In other words, horizontal light waves will pass through multiple horizontal filters (see Figure 1.1A), and vertical light waves will pass through multiple vertical filters (see Figure 1.1B). However, when two polarising filters are placed at right angles to each other, all light waves are blocked and no light can pass through (see Figure 1.1C). For instance, if the first filter was vertical, it would allow the vertical waves through while blocking the horizontal waves. Thus only vertical waves would reach the second filter; if the second filter was aligned horizontally it would block these vertical waves. The first filter already blocked the horizontal waves, thus no remaining light waves would pass through the second filter. $3 \mathrm{D}$ movies are created by polarising the images of two different movies in opposite directions, so that a different image can be presented to each eye.

${ }_{3} D$ movie presentation. In real life, depth perception occurs because the image each eye sees is slightly different-this difference occurs because of the distance between people's eyes. To create the impression of depth, slightly different images can be presented to each eye-the resulting disparity creates the illusion of depth. To create $3 \mathrm{D}$ movies, two movies are filmed from slightly different angles to simulate the different perspective of each eye. The two movie images are polarised—one horizontally and one vertically—by placing a polarising filter 
in front of each projector lens. The viewer wears polarisation glasses; one lens is aligned vertically to allow the vertically polarised image through while blocking the horizontally polarised image, and the other lens is aligned horizontally to allow the horizontally polarised image through while blocking the vertically polarised image (see Figure 1.2). The end result is that the viewers' eyes each see slightly different movies, and the differences create an illusion of depth so that certain parts of the movie appear to pop out from the screen.

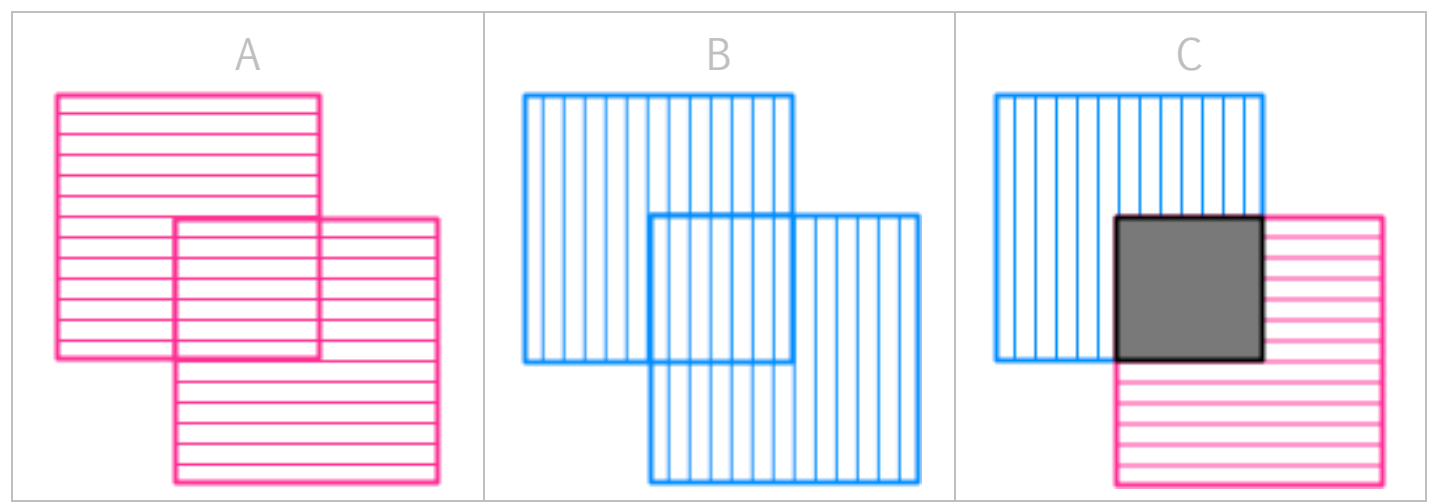

Figure 1.1. Illustration of polarisation filters. Horizontally aligned polarisation filters allow only horizontal light waves through (A); Vertically aligned polarisation filters allow only vertical light waves through (B); Polarisation filters placed at right angles to each other block all light waves (C).

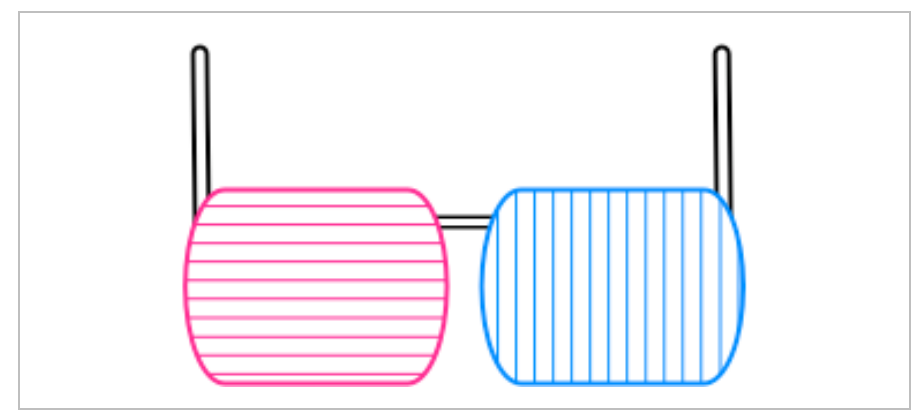

Figure 1.2. Illustration of polarising glasses worn to watch ${ }_{3} \mathrm{D}$ movies; one lens is made from horizontal polarising filter, and the other is made from vertical polarising filter. Two movies are polarised in different directions, thus each eye sees one version of the movie while the polarising filter blocks the other version. 
Mori and colleagues adapted the technique used to present $3 \mathrm{D}$ movies; instead of showing different movies to different eyes, they showed different movies to different viewers. Rather than using glasses with two lenses aligned at right angles to each other, Mori created new glasses with either two horizontally aligned polarising filters or two vertically aligned polarising filters (see Figure 1.3). Thus one pair of glasses would allow the viewer to see the vertically polarised movie, while blocking a horizontally polarised movie, and the other pair of glasses would do the opposite. Mori and colleagues named their new technique the Manipulation of Overlapping Rivalrous Images (MORI) technique (Kanematsu et al., 2003; Mori, 2003; Mori 2007).

If effective, the MORI technique would overcome the limitations of other work on memory conformity. Specifically, two viewers would be able to share the experience of watching an event together, but would each see a slightly different event. As a result, controlled systematic discrepancies should arise during a normal discussion between people who feel that they have experienced an event together-both in proximity and real time.

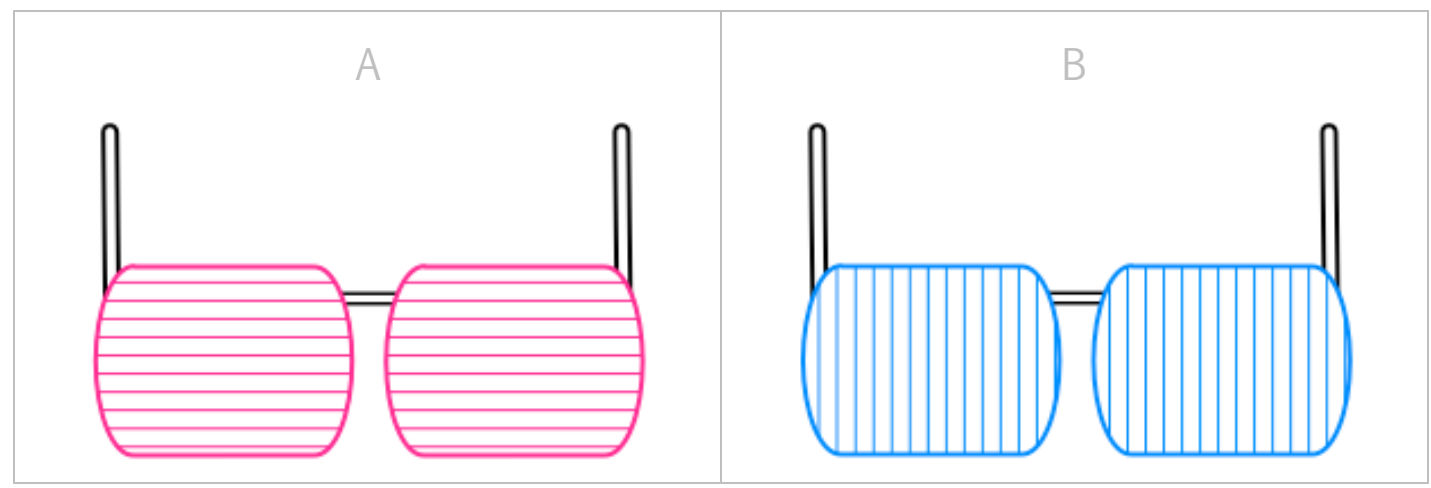

Figure 1.3. Illustration of polarising glasses worn for MORI technique; lenses are either both made from horizontal polarising filter (A); or both made from vertical polarising filter (B). Two movies are polarised in different directions, thus each viewer sees one version of the movie while the polarising filter blocks the other version. 
Research Using the MORI Technique

Mori and colleagues have conducted a number of studies on eyewitness memory using the MORI technique. These studies follow the same basic procedure. For example, in the original study Kanematsu et al. (2003) filmed two versions of a movie. In the movie a pedestrian is standing on the side of a road when a car pulls up. The driver gets out and asks for directions, while the passenger sneaks out of the car and steals the pedestrian's bag. Finally the car drives off, and the pedestrian walks away. There are three critical differences between the two movie versions: the colour of the car, the driver's clothes, and the direction the pedestrian walks in.

Kanematsu et al. (2003) filmed the two movies separately, and made adjustments to the timing of the movies so they overlapped as much as possible. The two movies were displayed on a rear projection screen, with the images from the two projectors set up to overlap. One movie was polarised horizontally and the other vertically. Subjects took part in the study in pairs-each wearing Mori's new polarisation glasses, under the guise that the glasses would simulate nighttime vision. The glasses allowed the wearer to see the image from one projector while blocking the image from the other projector (see Figure 1.4).

After watching the movie, subjects remembered the event individually before discussing it with each other. Control subjects then filled out another independent report, whereas the experimental subjects were asked to make a unified report with their discussion partner; subjects were required to agree on the answers for this unified report. Finally, one week later, subjects completed another memory test individually. Thus the researchers could compare subjects' initial responses with their later responses to examine what effect discussion had on their memories. 


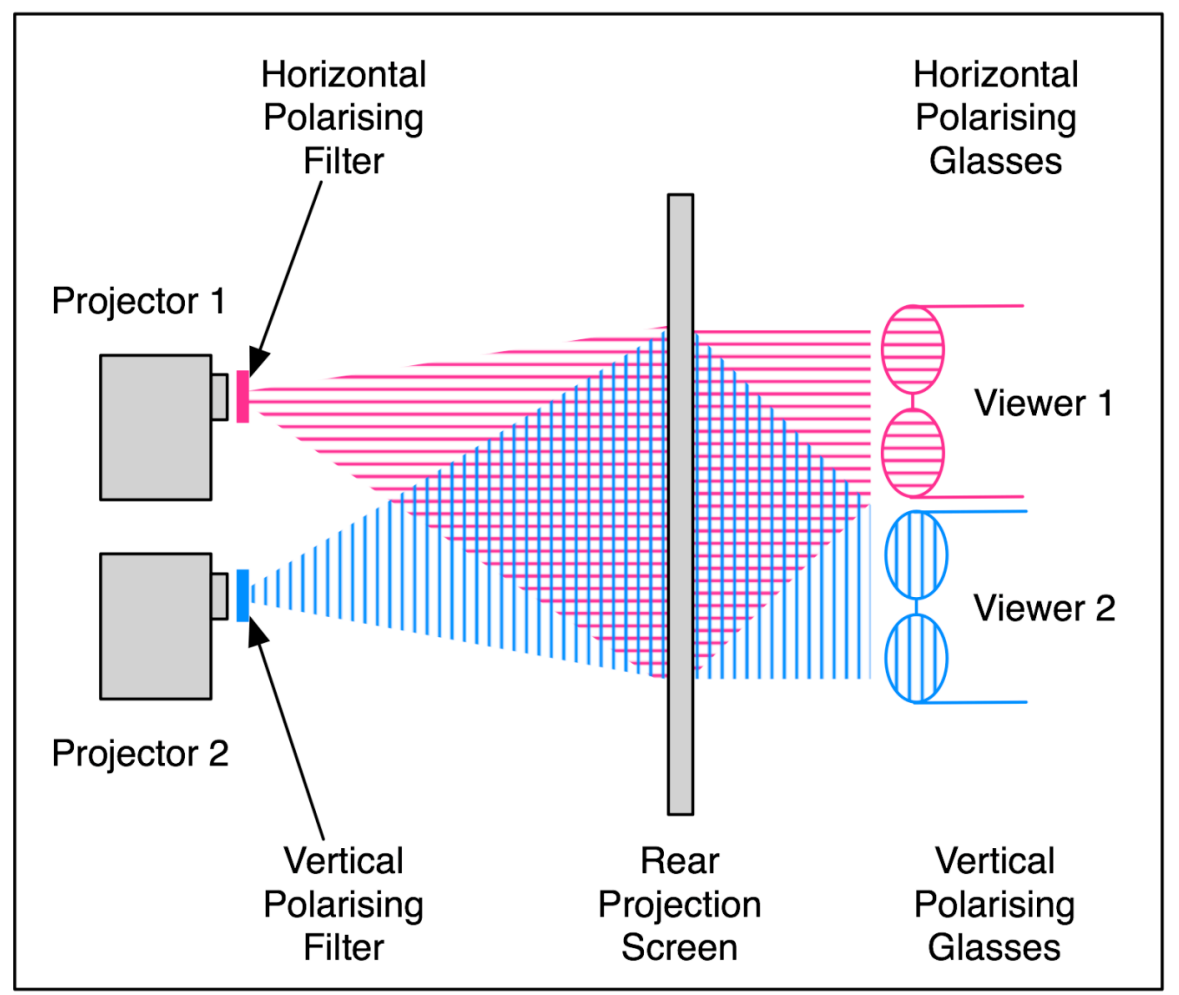

Figure 1.4. Illustration of equipment set up for MORI technique. Two projectors project two movies onto the screen. One movie is polarised horizontally, and the other is polarised vertically. Viewers wear polarisation glasses allowing them to see one movie while blocking the other.

Consistent with other research, subjects incorporated elements of each other's recall into their own memory reports after discussion. However, these studies too have their shortcomings. As described above, subjects in these studies completed a number of repeated memory tests, including —in some cases—a unified report where subjects were required to agree on their answers. This situation may not be a very good parallel to real life eyewitnesses who would—hopefully—never be asked to agree on their answers.

Another issue with these studies is that most use the movies that were developed for the original Kanematsu et al. (2003) study. Unfortunately however, there are some problems with these movies. Some research suggests that people cannot see or remember the critical details from the movies very well. For example, in one study, when subjects completed the individual memory test 
immediately following the movie, only 9 of 60 subjects correctly remembered the driver's clothes from their version of the movie (Mori \& Mori, 2006). If people cannot remember the critical details of the movie to begin with, it dramatically decreases the chance that they will mention those details during discussion with another subject, and increases the chance that they will accept the information that their partner tells them during discussion.

Finally, all the studies that have used the MORI technique to date have been based on data collected with Japanese subjects (for example, Hirokawa, Matsuno, Mori, \& Ukita, 2006; Itoh, 2006; Itoh, Umeda, \& Kawaguchi, 2005; Mori, 2005; Mori \& Kitabayashi, 2006). But these results might not translate well to Western samples: Japan is widely cited as a country that places emphasis on collectivist values (for example Kowner \& Wiseman, 2003; Sun, Horn, \& Merrit, 2004; WadeBenzoni, Okumura, Brett, Moore, Tenbrunsel, \& Bazerman, 2002). In a metaanalysis of Asch-type social conformity studies, subjects in Western individualistic cultures demonstrated much less conformity than those in collectivist cultures (Bond \& Smith, 1996). Similarly, a recent analysis of socially desirable responding across cultures found that collective cultural orientation was associated with subjects striving to maintain social relationships and gain acceptance by conforming to others. In contrast, individualistic cultural orientation was associated with responding to emphasise self-reliance (Lalwani, Shavitt, \& Johnson, 2006). Given that Japanese culture values conformity more than Western cultures, and that subjects were required to create a mutual report during discussion, it could be that the results thus far are due to response biases exacerbated by cultural values.

One final limitation of the memory conformity studies mentioned so farincluding the MORI technique studies_-is what or who makes up the control group. In some studies subjects complete repeated individual memory tests, 
while in others control memory performance is based on a second group of people who do not discuss the events together at all. The first design runs the risk of making the study goals very transparent, and increases the risk of response bias. The second design mimics that of the early misinformation effect research, where some subjects are given a narrative with misleading information and others are not. More recent misinformation effect studies use within-subjects designs, counterbalancing items so that each subject in the study is exposed to misleading information for some critical details but not for others. Using this method, researchers can look at subjects' baseline memory performance for critical details when they have not encountered any misleading information, and can examine what role that baseline memory might play in memory distortion. The MORI technique provides a means to introduce such counterbalancing into research on discussion and memory. By using the MORI technique, researchers will be able to introduce controlled errors into natural discussions between people who have the experience of a shared history to reconstruct together. If effective, the MORI technique provides the means to investigate a wide range of social factors and the role they play in memory distortion during social interactions. Put another way, the MORI technique may provide an opportunity to examine the factors that lead people to be more or less influenced by information conveyed in a discussion.

\section{Source Monitoring Framework}

Taken as a whole, the research on discussion and memory shows that people's memories are influenced by discussion with other people. But why are people's memories affected by what other people tell them? These results-and the results of misinformation effect research—can be explained by the Source Monitoring Framework (SMF; Johnson, Hashtroudi, \& Lindsay, 1993; Lindsay, 2008; Mitchell \& Johnson, 2000). According to the SMF, when people remember 
information, it does not come to mind with a label telling them where it came from. Instead, people rely on the qualitative characteristics of the remembered details (for example, the amount of perceptual information, vividness, tone of the event) and their knowledge and beliefs (for example expectations, judgments of plausibility or credibility) to decide where the details came from. Judgments about the quantity and quality of these different factors are made along a continuum, and different factors have different importance in different source monitoring decisions (Johnson, Foley, Suengas \& Raye, 1988).

According to the SMF, source monitoring processes are generally automatic; people often make source judgments—as they remember-without realising. However, in some situations people make a more deliberate effort to determine the sources of their memories-consciously weighing up their knowledge, beliefs and the characteristics of their memories. People do source monitoring to decide whether something really happened or whether they only thought about or imagined it (for example, "Did I fly to the moon yesterday or was it only a dream?" and "Did I unplug my hair straightener this morning, or did I only think about it?") and to decide which of two (or more) sources information came from (for example, "Was it Mollie or Jack who told me that joke?").

In more automatic source monitoring people might rely on qualitative characteristics such as how familiar or vivid a memory feels to inform them where it came from. In more deliberate source monitoring, people use their knowledge and beliefs as well (Johnson et al., 1993; Lindsay, 20o8; Mitchell \& Johnson, 2000). For example, I know that it takes a long time to travel to the moon so I can reason that it is quite unlikely I traveled all the way there yesterday. Additionally, I might recall that the tooth fairy flew to the moon with me; because I don't believe in the tooth fairy, I might reason that it is even more unlikely that the event I remember really happened. As a result of consciously 
weighing up all this information I can conclude that I only dreamed about flying to the moon and it never really happened.

Whether automatic or deliberate, the source monitoring process is far from perfect, and is prone to errors (Johnson et al., 1993; Lindsay, 2008; Mitchell \& Johnson, 2000). Put simply, a source monitoring error occurs when someone attributes information to the wrong source. In memory conformity studies, people make source monitoring errors by reporting the misleading details their discussion partner suggested instead of reporting the details they actually saw in the event. Similarly, people make source monitoring errors in misinformation effect studies by reporting misleading details from the post-event narrative or post-event questions instead of reporting what they saw.

These errors can occur in several ways. People might miss the information in the event and so rely on post-event information to fill the gaps. Alternatively people might remember aspects of the details from both the event and the discussion but confuse which information came from where. This confusion could result from either automatic or deliberate source monitoring. For instance, Jack might automatically decide that a specific detail came from the actual event because it feels very familiar to him-yet the familiarity might be due to the detail being mentioned in the discussion and thus being considered more recently. On the other hand, Mollie might struggle to decide if the hat she saw was black or blue, but she knows that Jack said it was blue. For whatever reason, Mollie might decide that Jack's description is more reliable than her own memory and so she reports blue. In this case Mollie took the misinformation to be evidence of reality.

Source monitoring errors in these types of studies might mean that subjects genuinely_but falsely_remember seeing the inaccurate details they were told about, or might mean that subjects have simply decided to report the details 
their partner suggested (whether strategically or not) even though they have no memory of those details. Either way, the end result is that people report seeing something that they did not see. For the remainder of this thesis, I will take people's independent test responses as evidence of their memories.

In the SMF, people use their knowledge, beliefs, schemas, and stereotypes to inform their source decisions (for example see Cook, Marsh \& Hicks, 2003; Hicks \& Cockman, 2003; Johnson et al. 1993; Lindsay, 20o8; Mather, Johnson, DeLeonardis, 1999). For instance, people are more likely to attribute information to a given source if the information fits with their stereotypes or knowledge about the source (Cook et al., 2003; Hicks \& Cockman, 2003; Mather et al., 1999). Additionally, people are more likely to attribute information to a source if the tone of the message fits with their expectations about the source (Holtgraves, Srull, \& Socall, 1989). As well as using social judgments about the source to decide where information came from and how it was delivered, people use social judgments to decide the value of a given piece of information (Johnson et al., 1993; Lindsay, 2008; Smith \& Ellsworth, 1987).

Although it is widely accepted that judgments about the source of information are used to guide source monitoring decisions-in order to target specific factors-research that examines social aspects of source monitoring tends to remove the source from the social context that it belongs to. For example, instead of having a conversation with another person, subjects might read statements that the person supposedly made; to examine the role of social judgments, subjects might be told that the person is a member of a certain category (for example, a lawyer), or might be given information that implies certain attributes (for example, trustworthiness; see Cook et al., 2003; Dodd \& Bradshaw, 1980; Echterhoff, Hirst, et al., 2005; Hicks \& Cockman, 2003; Holtgrave et al., 1989; Johnson et al., 1998; Mather et al., 1999). 
In real life conversations-whether deliberately or not-people make social evaluations of each other; but these judgments are not as simple as deciding that a given source is positive or negative, or fits into one category or another. Instead these judgments might be made on a variety of dimensions simultaneously (for example, the speaker's age, intentions, confidence, attractiveness, trustworthiness, occupation). These types of judgments might operate differently as a conglomerate in a social interaction than they would when analysed separately in a non-social context.

In fact, some research suggests that misinformation conveyed in a conversation maybe even more influential than misinformation encountered in other ways (Gabbert et al., 2004; Paterson \& Kemp, 2006b). Other research suggests that people might not always be influenced by a conversational partner's suggestions (Itsukushima et al., 2006; Vornik et al., 2003); instead they might weigh up a multitude of social dynamics within the conversation to decide the value of a given piece of information. In other words, the unique social dynamics of the conversation might work to increase or decrease the chance that someone will be influenced by misinformation. These studies suggest that-to fully understand social influences on source monitoring-it is important to examine the social context. In short, social aspects of people's interactions might affect their source monitoring behaviour, and as such are an important feature of the SMF to investigate further.

\section{Thesis Overview}

My overarching aim was to build on this body of research in several ways. In this thesis, my major goal was to investigate how people's judgments of their conversational partners might affect their source monitoring behaviour and thus their susceptibility to misleading suggestions conveyed in a conversation. I used the MORI technique to present pairs of people with two slightly different 
versions of an event to discuss together. Before addressing my primary goal, I first report six experiments that examine the effectiveness of the MORI presentation technique for conducting research on discussion and memory. In the second part of the thesis, I report three experiments that examine how different social dynamics contribute to memory distortion in social interactions.

In Experiments $1 \mathrm{~A}$ and $1 \mathrm{~B}$ (Chapter 2) I aimed to establish appropriate materials for use with the MORI technique. To that end I conducted two pilot misinformation effect studies, using Takarangi et al.'s (2006) misinformation materials. I tested subjects' ability to remember the critical details from the movies. If subjects' baseline memory performance was above chance it would demonstrate that subjects could see and remember the critical details. Better memory for control details (where subjects were not exposed to misinformation) than misled details (where subjects were exposed to misinformation) would show that subjects' memories were influenced by misinformation conveyed in a narrative description.

In Experiments $2 \mathrm{~A}$ and ${ }_{2} \mathrm{~B}$ (Chapter 3), I investigated whether subjects' memories were influenced by misinformation mentioned by another person. Instead of providing subjects with misinformation in the form of a narrative description, subject-pairs discussed some critical details together. If subjects were better at remembering nondiscussed (control) details, than discussed (misled) details, it would provide evidence that they were influenced by misinformation conveyed in a discussion. In Experiment 2A I used an adapted version of Takarangi et al.'s (2006) misinformation materials; in Experiment $2 \mathrm{~B}$, I developed a new target event and stimulus set to generalise the results beyond Takarangi et al.'s materials.

In Experiments $3 \mathrm{~A}$ and ${ }_{3} \mathrm{~B}$ (Chapter 4 ), I aimed to rule out counterexplanations of the results. Specifically, I wanted to investigate whether 
subjects could see the alternate version of the movie- the version that was supposed to be blocked. In Experiment 3A I asked increasingly suggestive questions to examine whether subjects would report seeing the alternate movie version. For half of the subjects, both movies were played and overlapped via the MORI technique; for the other half only one version was played, so there was no overlapping image to notice. If subjects in the overlap group did notice the other version of the movie they should be more likely to say so than subjects in the non-overlap group. If both groups were equally likely to say they saw two versions it would suggest the results were due to the demand characteristics of the questions.

In Experiment 3B I used a second-choice test to examine whether subjects had any implicit memory for the alternate movie. Subjects watched half of the movie (and half of the critical details) while the other version was played and blocked via the MORI technique; for the remaining critical details the other movie version was not played so there was no overlapping image. If subjects had any implicit memory of the alternate movie, they should be more likely to pick the correct answer from that version for critical details that were overlapped than for critical details that were not overlapped.

In Experiment 4 (Chapter 5), I examined whether people's relationships with each other affected their susceptibility to false memories. Subjects took part with either their romantic partner or a stranger. If relationship features such as trust and respect affected subjects' source monitoring decisions, then subjects who took part with their romantic partner should be more influenced by the misinformation than subjects who took part with a stranger.

In Experiments 5 and 6 (Chapters 6 and 7), I was interested in whether subjects' expectations about their own abilities and about their discussion partner's abilities might affect their source monitoring decisions. To address this 
question I told subjects that they either had the same, better or worse ability to see the movie than their partner did. If subjects' expectations about their own abilities affected their source monitoring decisions, those subjects who had high expectations of themselves might be less influenced than those who had low expectations of themselves. Alternatively, if subjects' expectations about their discussion partner-the misinformation messenger-affected their source monitoring decisions, those subjects who had high expectations of their partners might be more influenced than those who had low expectations. Finally, if subjects' expectations about themselves and their expectations about the misinformation messenger both affected their source monitoring decisions, those subjects who expected to be better than the messenger might be less influenced, and those who expected to be worse than the messenger might be more influenced. 


\section{Chapter 2}

\section{Experiment 1: Misinformation Materials and the MORI Technique}

As discussed in Chapter 1, there is some evidence that-at least with the Kanematsu et al. (2003) movie-people may not be able to see details very well when images are presented via the MORI technique (see Mori \& Mori, 2006). One possible cause of this problem is that the technique requires researchers to present the movies on a semi-transparent rear-projection screen, and as a result the image quality is not as sharp or bright as people might be used to. My first goal was to test whether people were able to make out and remember the details of a movie presented via the MORI technique. To that end, I conducted two pilot studies using Takarangi et al.'s (2006) misinformation materials.

Takarangi et al. (2006) developed a completely counterbalanced set of misinformation materials made up of two versions of a movie, and four versions of a corresponding narrative. The movie shows an electrician named "Eric" working in an unoccupied house. During the movie Eric strays from his duties, wanders around the house, delves into personal possessions, and steals various items. The movie is identical across both versions except for eight critical differences (see Table 2.1). Takarangi et al. filmed only one version of the movie, and then digitally altered it to create the eight critical differences. For example, in one version of the movie Eric stops to look at a picture of the Leaning Tower of Pisa, and in the other he looks at a picture of the Eiffel Tower instead.

Takarangi et al.'s (2006) study followed the basic misinformation effect method described in Chapter 1. Subjects watched one version of the movie, then read a narrative that described the movie. The narrative contained misleading information about some of the critical details, but not others. Finally, subjects completed a recognition test to see how well they remembered details from the movie. Takarangi et al.'s results showed a large misinformation effect: subjects 
were worse at remembering the misled details, details for which they were exposed to misinformation in the narrative. In other words, on the test, subjects reported what they read in the narrative rather than what they saw in the movie.

Takarangi et al.'s (2006) materials are ideal for use with the MORI technique because the two versions overlap perfectly at all points except during the presentation of the eight critical details. Recall that Kanematsu et al. (2003) filmed their two movies separately, and made adjustments to the speed of the movies in order to get the versions to overlap as much as possible. One common concern that reviewers have about the MORI technique is that if subjects tilt their heads during the movie presentation, the angle of the polarisation will change, and they will be able to see the "blocked" image. However, if the two images match perfectly—as Takarangi et al.s do-and they are played in time with one another, then even to the naked eye for the vast majority of the movie it would appear that only one movie was being played. Thus, if subjects did tilt their heads at some point during Takarangi et al.'s movie, it is very unlikely that they would notice the two images. In short, the design of Takarangi et al.'s materials should work well with the MORI technique.

The primary purpose of these pilot studies was to test whether subjects could see and remember the critical details of a movie presented via the MORI technique. If subjects were able to see and remember the critical details, their baseline memory performance should be better than chance levels on the final recognition test. Additionally, if subjects could remember the critical details of the movie, I wanted to examine whether those memories were susceptible to distortion. If subjects' memories were influenced by misinformation, then they should be better at remembering details for which they saw no misinformation (control details) than details for which they saw misinformation (misled details). 
Table 2.1

Critical differences between Takarangi et al.'s (20o6) movie versions

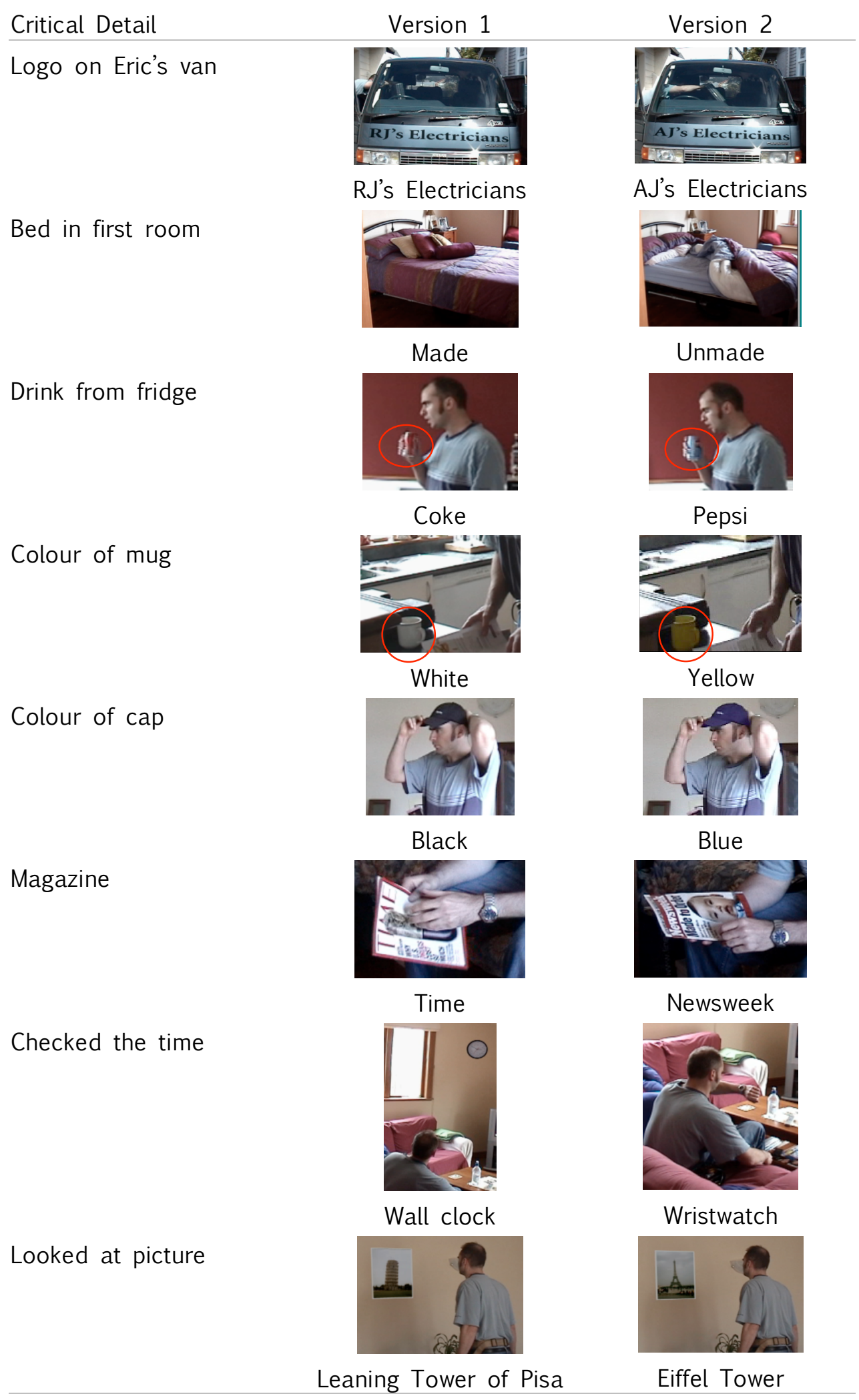




\section{Experiment $1 \mathrm{~A}$}

Method

\section{Subjects}

In total, 16 students from the Introduction to Psychology Research Programme (IPRP) pool took part in return for course credit. Each session had either one or two subjects.

Design

I followed Takarangi et al.'s (2006) method, but used the MORI technique to show subjects the movie. Regardless of the number of subjects per session, both movie versions were played; each subject watched one version of the movie while the other version was blocked.

\section{Materials and Procedure}

Subjects came to the laboratory and sat down at a table facing the rear projection screen. As a cover story, I told subjects that the study was about people's sensory impressions at different levels of visual acuity. More specifically, at the beginning of the session I told them:

We are interested in people's sensory impressions at different levels of visual acuity. Visual acuity basically means how well you can see. So, right now you should have $100 \%$ visual acuity either because your eyes work properly, or because you have correcting lenses on. We want to know what happens to people's sensory impressions when their visual acuity is degraded by different amounts.

While explaining the study, I brought the subjects' attention to a stack of four boxes containing "acuity glasses.” Each box was labeled as though it contained different strength acuity glasses; for example, one box read "95\% Acuity Glasses: 4 pairs" (see Figure 2.1A). In fact, only the "95\% Acuity" box contained glasses; the other boxes were empty, but were displayed to add authenticity to the cover story. Next, I told subjects:

Today, you will be in the $95 \%$ acuity condition, so that's not too different to normal 
vision. I will give you a pair of $95 \%$ acuity glasses and I am going to show you a movie of a tradesman named Eric working in a house; I just want you to watch the movie through the glasses, and then we will do a couple of related tasks afterwards. You might find that the movie looks a bit funny through the glasses, but don't worry-that is basically how they are supposed to work. We do find that people see best when they keep their head straight rather than tilted, so we ask that you keep your head as straight and still as possible while you watch the movie. If you already wear glasses, the acuity glasses should fit over the top.

I gave each subject a pair of polarisation glasses from the $95 \%$ acuity box (see Figure 2.1B). There were three phases to the study: first subjects watched the movie, next they read a narrative about the movie, and finally, they completed a surprise memory test. ${ }^{1}$
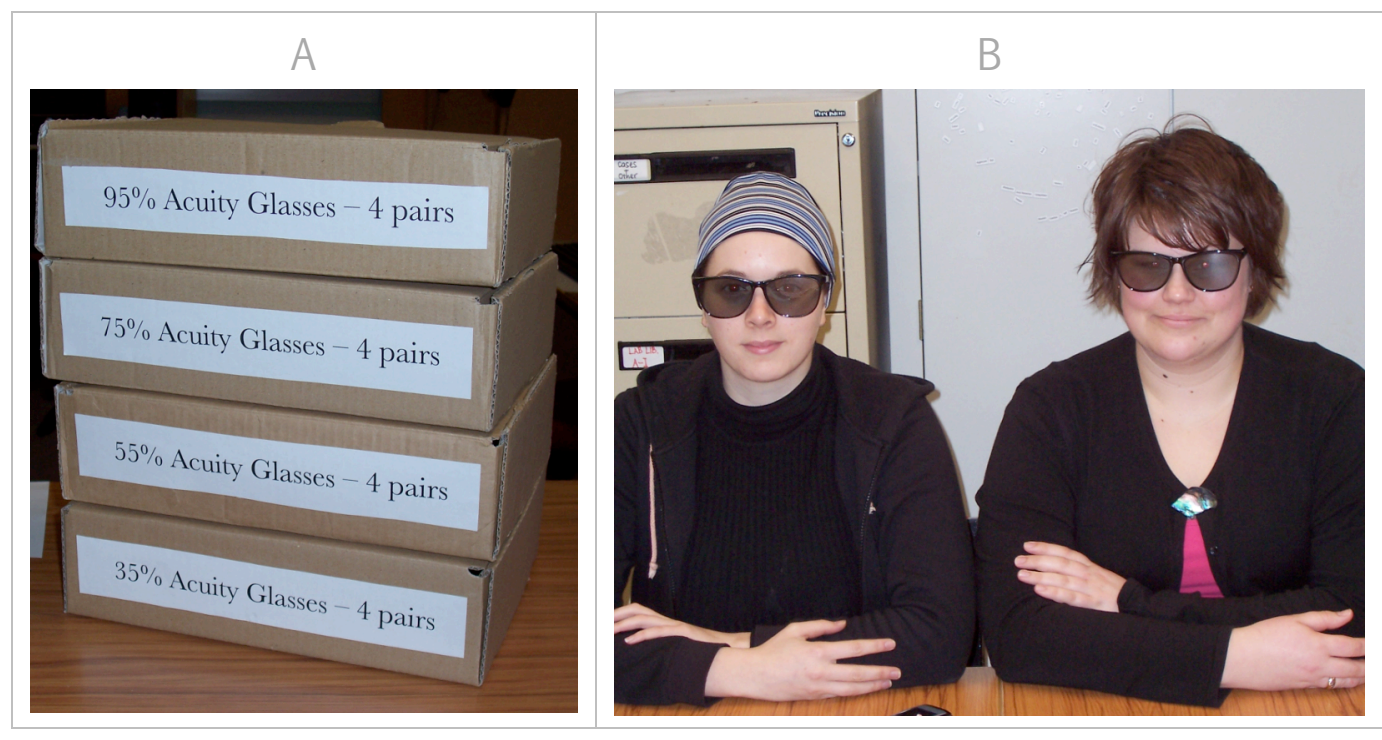

Figure 2.1. Photographs of boxes ostensibly containing different strengths of “Acuity Glasses” (A) and people wearing polarisation glasses (B).

Phase 1. The two movie versions were each played on a separate Apple iBook PowerPC G4; each iBook was connected to a NEC ViewLight Mobile DPL projector, model LT75ZJ, and the two images were projected onto the rear of a

\footnotetext{
${ }^{1}$ Examples of the materials used in this experiment and subsequent experiments appear in the Appendices.
} 
semi-transparent projection screen (see Figure 2.2A). The rear projection screen was made from a $1.22 \mathrm{~m} \times 1.82 \mathrm{~m}$ wooden panel and had a $29 \mathrm{~cm} \times 38 \mathrm{~cm}$ cutout holding the semi-transparent screen. All the equipment was hidden from subjects' view by the rear projection screen (see Figure $2.2 \mathrm{~B}$ ). Two projectors were mounted one above the other in a metal stand, each tilted toward the screen so that the two images overlapped. One movie was polarised horizontally by attaching a polarising filter horizontally to the projector stand in front of the lens. The other movie was polarised vertically by doing the same with another filter placed at 90 degrees (see Figure $2.2 \mathrm{C}$ and $2.2 \mathrm{D}$ ).

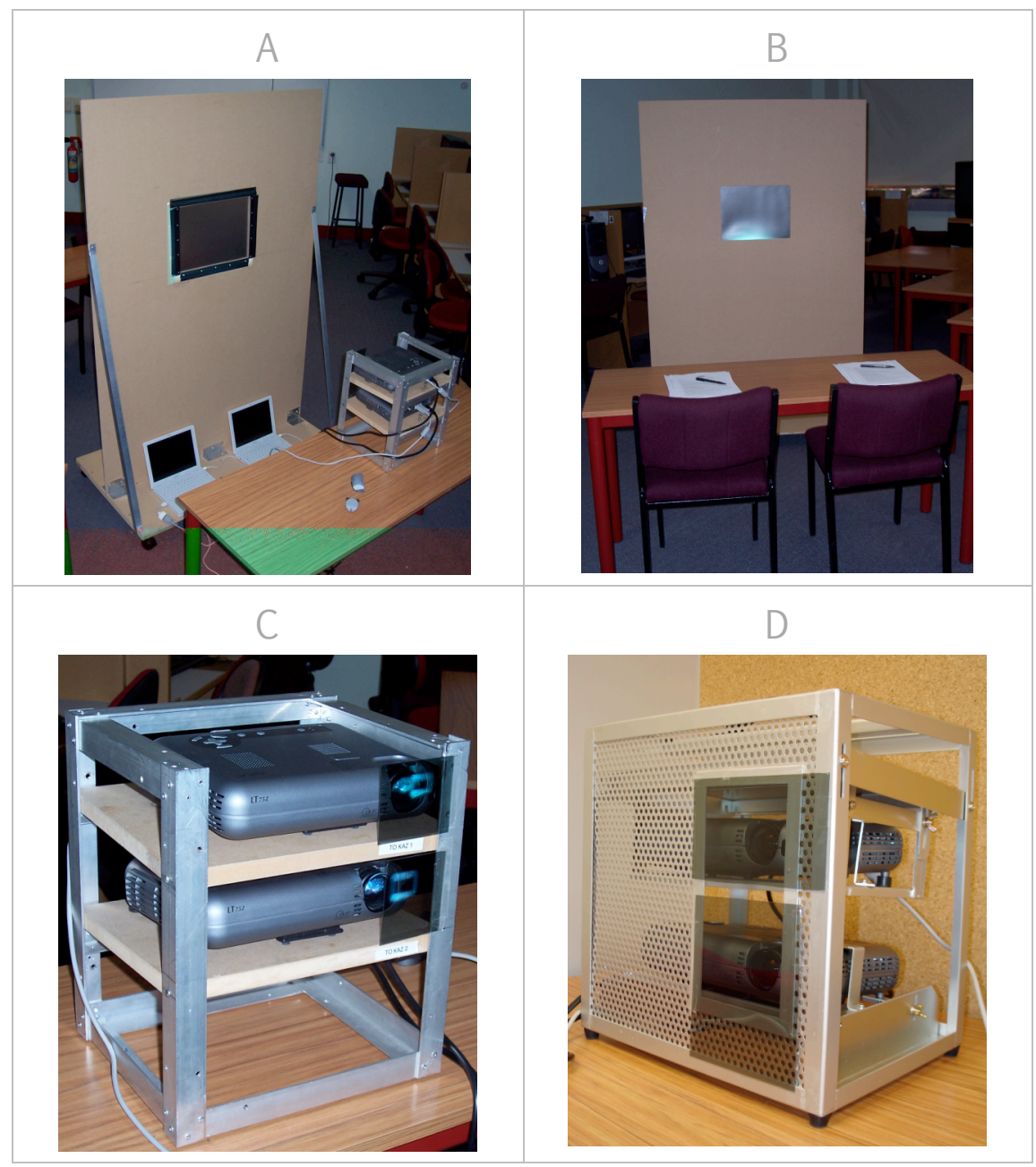

Figure 2.2. Photographs of equipment used for MORI technique: Behind the rear projection screen (A); from subjects' view, at the front of the projection screen (B); the projector stand and polarisation filter $(\mathrm{C})$ and the projector stand used in later studies (D). 
Each subject either wore glasses that allowed him or her to see the horizontally polarised image while blocking the vertically polarised image, or wore glasses that did the opposite. Subjects sat at a table facing the rear projection screen for the duration of the study. The movie(s) had no audio, and ran for 6:28 min. In order for subjects' memories to fade, and to prevent them from thinking about the movie, following the movie subjects completed a 15-min filler logic puzzle.

Phase 2. Next, subjects read one of four versions of the narrative description used in Takarangi et al.s (2006) study. The narratives were between 781 and 786 words long and were identical except for information about the eight critical details. Each narrative contained misleading information about four critical details, and generic information about the other four. The misinformation was counterbalanced to match the corresponding detail presented in the other version of the movie. For example, if someone watched Eric looking at the Eiffel Tower in the movie, the misleading information would say he looked at the Leaning Tower of Pisa, whereas the generic information would say he looked at a picture on the wall. Each critical detail acted equally often as a misled detail and as a control detail.

Phase 3. After completing another five-minute filler task, subjects were given a surprise memory test. I told subjects that the questions were testing their memory for the movie; this point was reiterated in written instructions at the start of the test. The test was taken from Takarangi et al. (2006); there were 20 two-alternative forced-choice recognition questions. Eight of the questions asked about the eight critical details; for these questions subjects were presented with the correct detail from each of the two movie versions. In other words, subjects chose between the correct detail from the movie they saw and the misleading detail from the other movie and narrative. For example "Eric looked at a picture 
of the tower" was followed by the two alternatives "Leaning" and "Eiffel".

The remaining twelve questions were fillers. After each question, subjects used a scale to rate how confident they were that their answer was correct, where $1=n o t$ at all confident, and $5=$ very confident .

Subjects were thanked for their time at the end of the session, and were fully debriefed when the study was complete.

Results and Discussion

Before addressing the primary purpose of the study—whether subjects could see and remember the critical details-I first examined how well they remembered the non-critical filler details: these details were the same in both versions of the movie. In fact, subjects were very good at answering these twelve questions on the test, and there were no differences between the movie versions, $M=11.00$ correct (92\%), $S D=0.93 ; M=10.88$ correct ( $91 \%), S D=1.55, t<1, n s$.

\section{Remembering Critical Details}

I next analysed subjects' performance on each of the control details, details for which they read only generic information. Here and throughout the thesis, memory performance is based on the proportion of correct responses for each subject. In a two-alternative forced-choice recognition test, chance performance is $50 \%$; thus to establish that subjects could see and remember the details from the movie, each detail should yield a proportion correct score greater than .50 (when acting as a control detail).

For each of the eight critical details, I calculated the proportion of correct control responses; these proportions are displayed in Figure 2.3. The red line illustrates chance performance (.50). As Figure 2.3 shows, for four of the eight critical details, subjects' baseline memory performance was at or below chance, suggesting that the movie was not clear enough for them to be able to make out and remember the critical details. Specifically, subjects were poor at 
remembering the bed, drink, mug, and cap details.

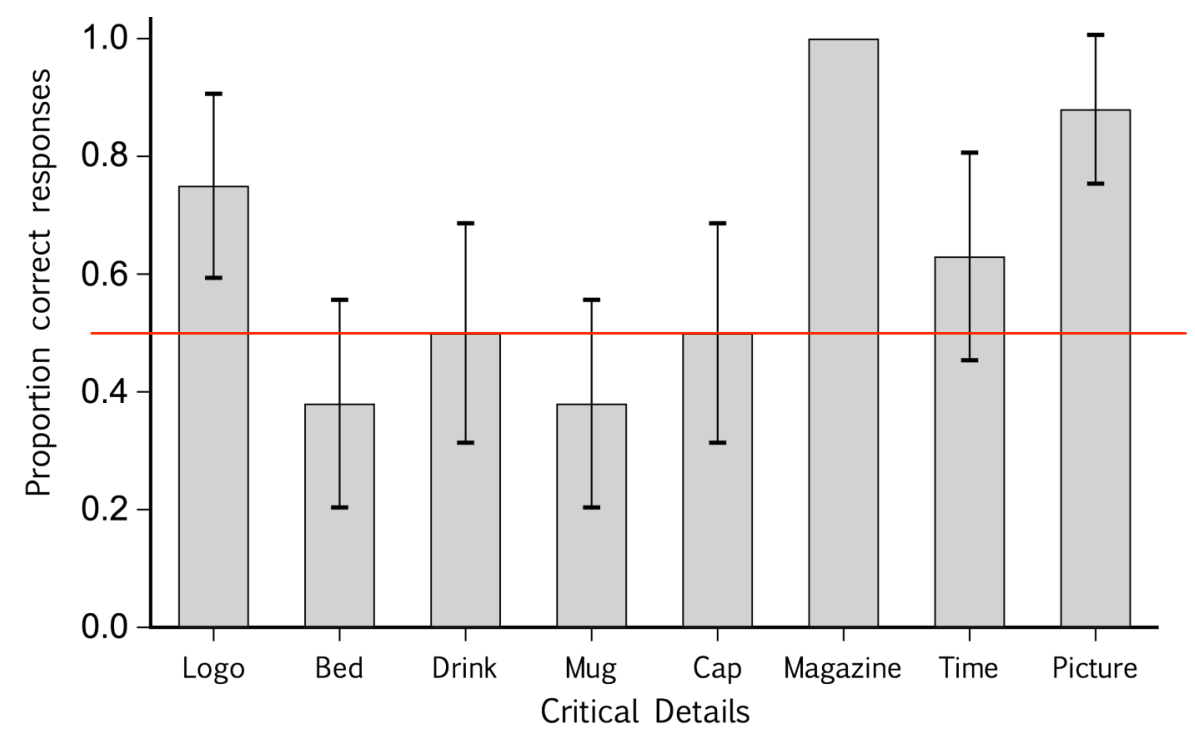

Figure 2.3. Proportion of correct control responses for each critical detail. Error bars depict standard errors of the means.

\section{Summary}

In short, subjects remembered the filler details but not the critical details. If subjects have poor memory for the critical details it is unlikely that those details would arise during discussion between the subject and another person. In Experiment $1 \mathrm{~B}$, I aimed to improve memory for these critical details.

\section{Experiment 1B}

I used Takarangi et al.s (2006) original files and the same software to make several changes to these problem details. Takarangi et al. (2006) created the two versions of the cap detail and the mug details by changing the colour of the original filmed material. For instance, the cap was originally blue; Takarangi et al. created a second version by changing it to black. Because the images on the semi-transparent screen were quite dark, I was concerned that the image wasn't clear enough for subjects to tell the whether the cap was blue or black, or whether the mug was white or yellow. I made these differences more 
pronounced by creating a new red version of the cap to replace the blue version, and a new green version of the mug to replace the yellow version. Table 2.2 depicts the new versions of the revised critical details. I changed the corresponding information in the narratives and on the test to reflect these new critical details. For the other two problem details (bed and $d r i n k$ ) I added extra footage to the movie so the critical details appeared onscreen for longer. The new versions of the movie ran for 6:34 $\mathrm{min}$.

Table 2.2

Updated critical details in revised versions of Takarangi et al.'s (20o6) movie

Critical Detail Version 1

Method

\section{Subjects}

Twenty IPRP students took part in return for course credit.

\section{Design}

The design was identical to Experiment 1A.

\section{Materials and Procedure}

The method and procedure were the same as Experiment 1A, except I used the revised movie versions, narratives and recognition test.

Results and Discussion

As in Experiment 1A, subjects performed very well on the twelve filler 
questions, and there were no differences in memory performance between the two versions of the movie $M=11.40$ correct (95\%), $S D=1.07 ; M=11.10$ correct (93\%), $S D=0.88, t<1, n S$.

\section{Remembering Critical Details}

Of most interest was subjects' baseline memory performance. As Figure 2.4 shows, the proportion of correct control responses was above chance for every detail. Collapsing across details, subjects were good at reporting what they had seen in the new movie versions, with an overall proportion of .75 correct responses for control details $(S D=.21)$.

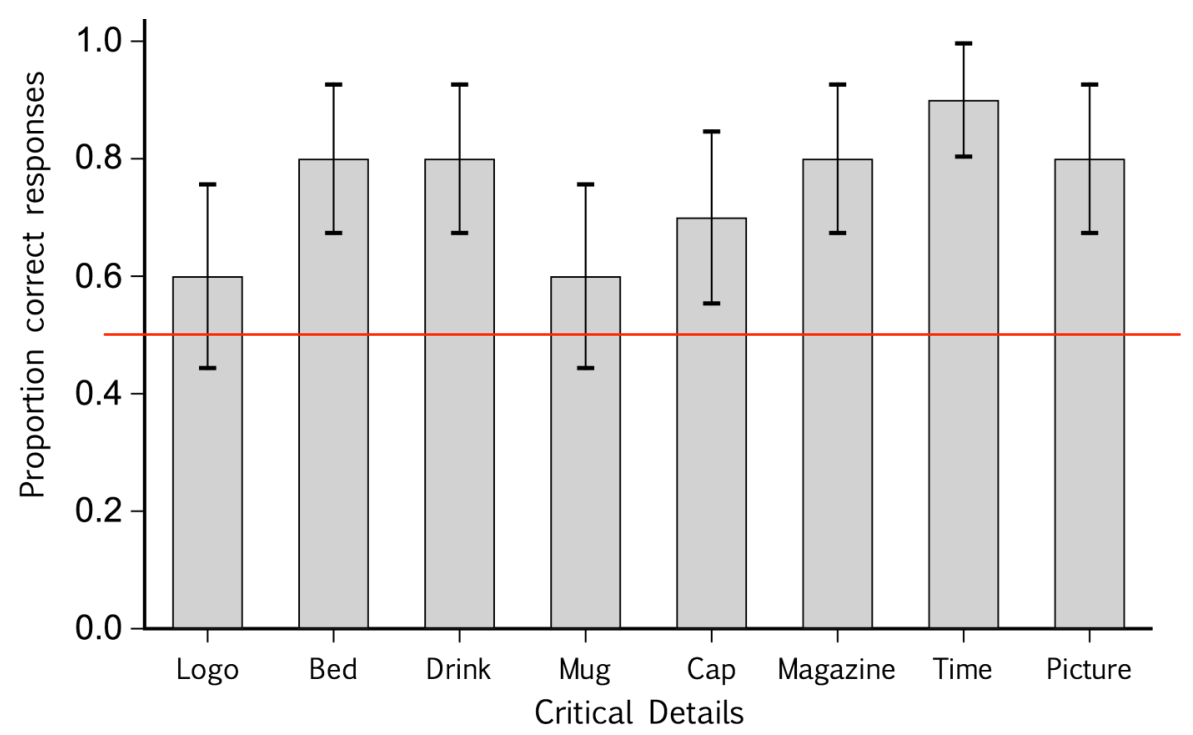

Figure 2.4. Proportion of correct control responses for each critical detail. Error bars depict standard errors of the means.

In short, subjects remembered the critical details from the revised movie versions. If subjects have good baseline memory for the critical details those details should arise during discussion between the subject and another person. In other words, using the revised Takarangi et al. (2006) materials might work well with the MORI technique in order to get subjects to expose each other to misinformation during a discussion. Before I could study how discussion might 
affect people's memories, I first needed to establish whether subjects' memories of the revised movie versions were susceptible to the influence of misinformation. If subjects' memories were especially clear, perhaps the misinformation would not influence what they remembered.

\section{Misinformation Effect}

To see if subjects' memories were affected by the misinformation contained in the narrative, I calculated the proportion of correct responses each subject gave for control details and misled details; these proportions are displayed in Figure 2.5. As Figure 2.5 shows, subjects were better at remembering control details than misled details, $t(19)=3.44, p<.01$, Cohen's $d=0.78 .^{2}$ In other words, subjects' memories were influenced by the misinformation contained in the narrative, and they incorrectly reported the misinformation instead of what they saw.

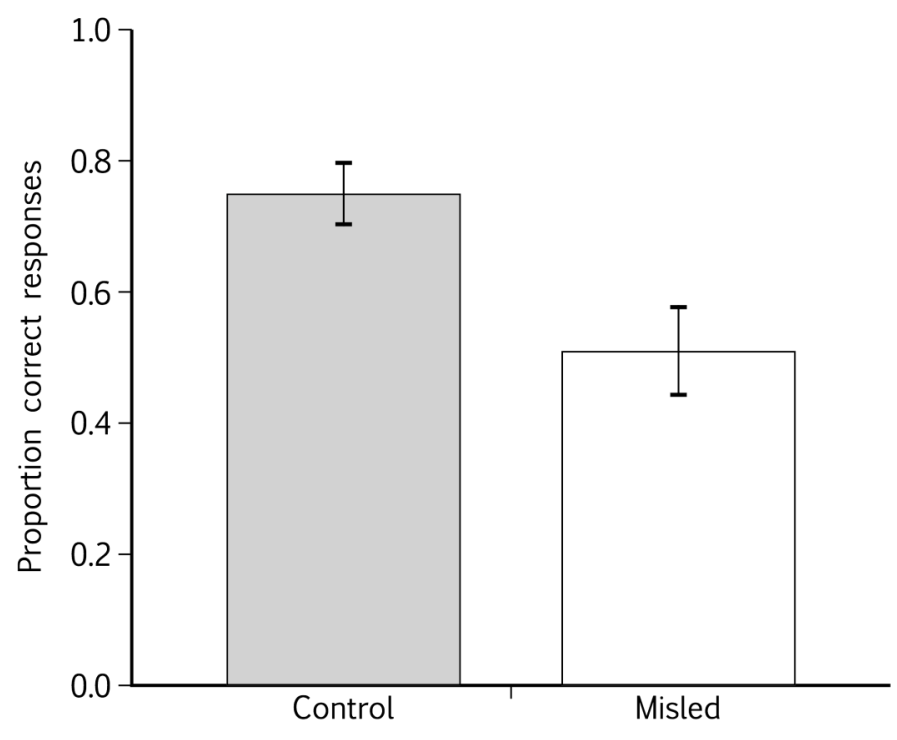

Figure 2.5. Proportion of correct responses for control and misled details. Error bars depict standard errors of the means.

Next, to examine the contribution of each critical detail, I calculated subjects' mean performance for each detail when it acted as a control detail and when it

\footnotetext{
${ }^{2}$ Effect sizes were calculated using G*Power v3.o.
} 
acted as a misled detail. These data are displayed in Figure 2.6. As the figure shows, people were more likely to report the correct answer for the critical details when they acted as control details, $t(7)=3.15, p<.01$, Cohen's $d=2.05$.

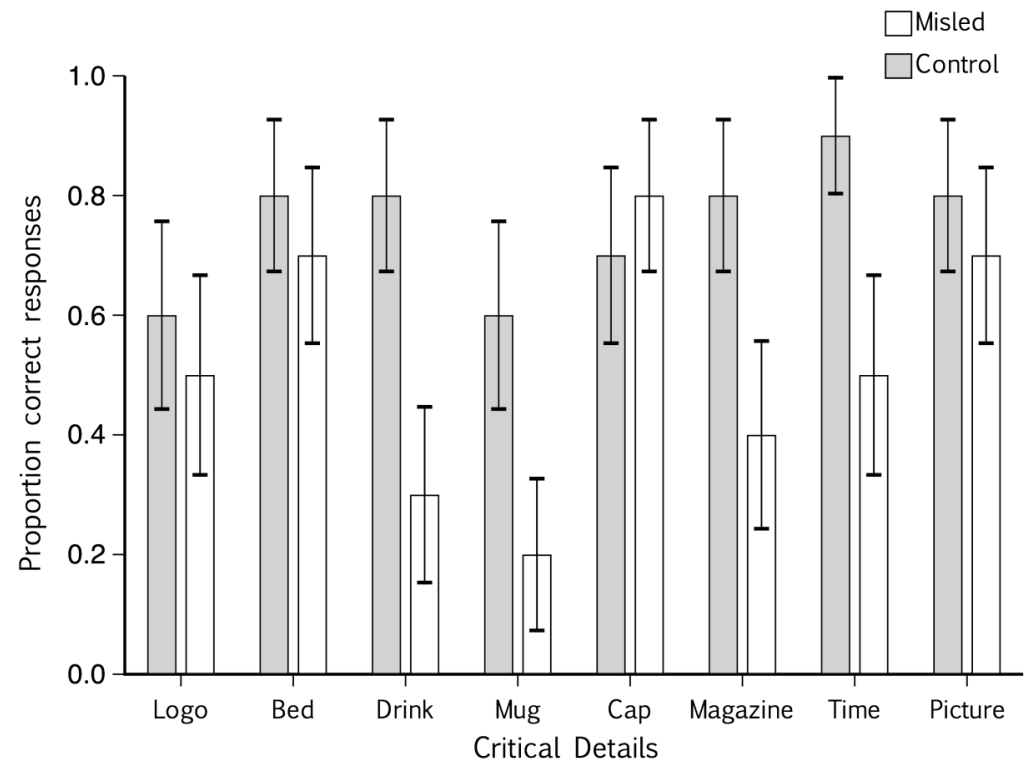

Figure 2.6. Proportion of correct control and misled responses for each critical detail. Error bars depict standard errors of the means.

\section{Confidence}

These results tell us about subjects' memory accuracy, but they do not tell us about subjects' subjective experiences of their memories. I next compared subjects' confidence ratings for their responses to control and misled details. As shown on Figure 2.7, subjects were moderately confident in all their responses, and slightly more confident in their responses to misled than control details, $t$ $(19)=2.54, p=.01$, Cohen's $d=0.56$. Taken together, the results so far suggest that reading misinformation hurt subjects' accuracy, yet boosted confidence in their memory reports. 


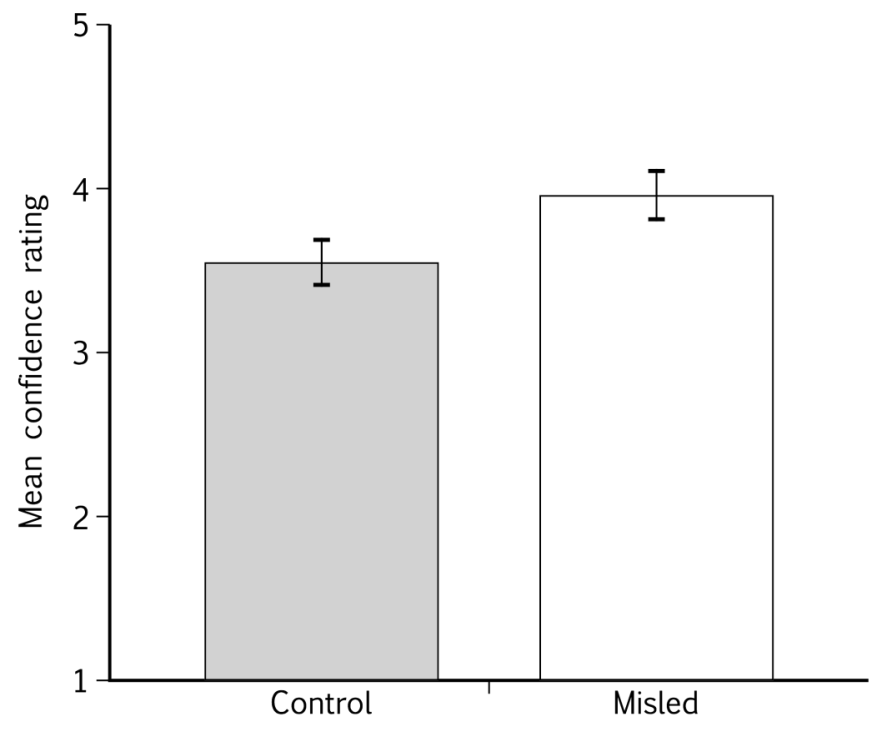

Figure 2.7. Mean confidence for control and misled details. Error bars depict standard errors of the means.

Another way to look at subjects' experiences of their memories is to examine confidence in their correct and incorrect responses. To that end, I calculated subjects' confidence when they said that they saw a detail in the movie, and compared subjects' confidence in their correct responses on control details (true memories) with their incorrect responses on misled details (false memories). As shown in Figure 2.8, subjects were equally confident in both types of responses: relying on the misinformation did not affect subjects' confidence in any way, $t$ $(16)<1, n s$. Put another way, subjects were just as confident in their false memories as they were in their true memories. 


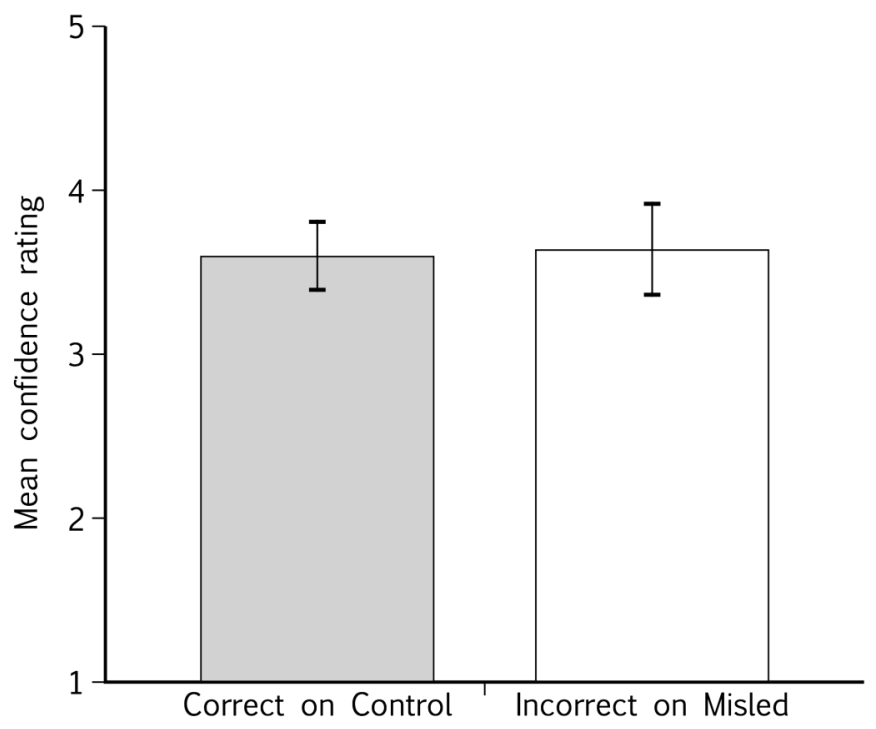

Figure 2.8. Mean confidence for correct responses on control details (true memories) and incorrect responses on misled details (false memories). Error bars depict standard errors of the means.

\section{Summary}

Considered as a whole, the data from Experiment $1 \mathrm{~B}$ suggest that the revised versions of Takarangi et al.'s (2006) materials work effectively with the MORI technique. Subjects remembered control details at a level higher than chance, but were still influenced by misleading suggestions. My next goal was to see whether I would get the same pattern of results if_-instead of reading a misleading narrative about the event—subjects discussed the event together. 


\section{Chapter 3}

\section{Experiment 2: Misinformation Delivered via Discussion}

Misinformation delivered in a narrative may be experienced in a qualitatively different way from misinformation delivered in a discussion. Specifically, information in a narrative might be ascribed some expert quality by virtue of it coming from the experimenter, or by virtue of the amount of highly detailed information in it (Johnson, Bush, \& Mitchell, 1998; Smith \& Ellsworth, 1987). Additionally, misinformation provided in a written form may miss the vast array of social dynamics that may be at play in a real life interaction. My next step was to examine whether people would be susceptible to misinformation conveyed by a peer during discussion about a movie presented via the MORI technique.

\section{Experiment 2A}

My main goal in this study was to build on the methodology of the previous MORI technique research described in Chapter 1 . To that end, I used a withinsubjects design so that each subject discussed some critical details but not others. Using a within-subjects design provided a means to make fair comparisons between subjects' memories for nondiscussed critical details and discussed critical details, parallel to the comparisons made between people's memories for control and misled details in a misinformation effect study. Additionally, in order to make these comparisons, subjects needed to complete only one memory test at the very end of the study; as mentioned in Chapter 1, in previous MORI technique research, subjects were required to complete repeated memory tests to examine their pre and post discussion memories. In short, the repeated tests might have made it obvious to subjects that the researchers were interested in how discussion shaped memory; by requiring subjects in my study to complete only one test, this transparency should be decreased.

If people are influenced by misinformation conveyed in a conversation by 
someone who has shared an experience, then subjects should be better at remembering nondiscussed details than discussed details.

Method

\section{Subjects}

In total, 40 IPRP students participated in pairs in return for course credit. Design

The study was a within-subjects design. For each subject, four of the eight critical details acted as discussed details, parallel to the misled details in a misinformation study; the other four acted as nondiscussed details, parallel to the control details in a misinformation study. Critical details were fully counterbalanced so that each served equally as a nondiscussed and discussed detail.

\section{Materials and Procedure}

The method was based on Experiment $1 \mathrm{~B}$ and had three phases.

Phase 1. First, subjects watched the movie as described in Chapter 2. One member of each pair watched one version of the movie, and the other person watched the other version. After the movie, subjects completed a 15-minute filler task.

Phase 2. In the second phase of the study, instead of reading a narrative description, subject-pairs were asked to talk about what they saw in the movie. In order for subjects to discuss some critical details, but not others, their discussions were guided by questions. Twelve discussion questions were presented one at a time on the rear projection screen using Microsoft PowerPoint ${ }^{\circledR}$; each question asked about a detail from the movie and provided five alternative answers (see Figure 3.1). Four of the twelve questions targeted critical details; for these four questions, the correct answer from each movie version appeared in the list of five alternatives (see Figure 3.1A). The other four critical details were not mentioned 
during the discussion, and served as the nondiscussed (control) details at test. The remaining eight discussion questions were fillers, and were not mentioned again (see Figure 3.1B).

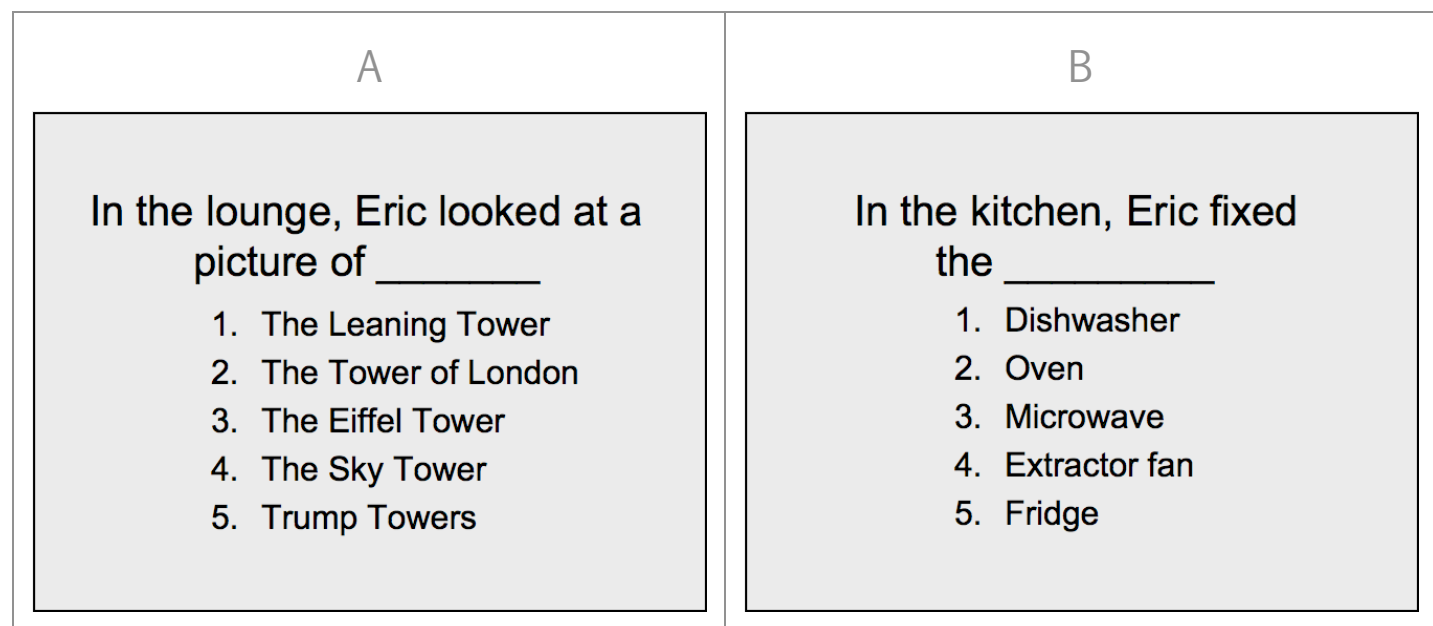

Figure 3.1. Example of slides used in discussion for critical questions (A) and filler questions (B).

The experimenter recorded subjects' conversations using an Olympus ${ }^{\circledR}$ DS 2000 digital voice recorder, and also noted subjects' answers during the discussion. Subject-pairs were asked to discuss the questions together, figure out the answer, and tell the experimenter what answer they decided on. If the subjects could not agree, they were permitted to give separate answers.

Pilot testing showed that pairs of people generally answered the questions within 30 seconds, so to ensure they had ample time, subjects were given up to one minute to discuss each question, and were warned when they had 10 seconds remaining. After subjects told the experimenter their answer(s), the experimenter displayed the next question. Following the discussion, subjects completed a filler task for five minutes.

Phase 3. Finally, subjects completed a surprise recognition test independently; the test was identical to the test used in Experiment 1B. For the eight critical questions, the two alternatives were structured so that subjects chose between the 
correct detail from their version of the movie and the correct detail from their partner's version.

\section{Results and Discussion}

Twenty pairs of subjects completed the study; however, all data-except for discussion duration-were analysed on an individual level, yielding a total of 40 subjects. ${ }^{3}$ Consistent with Experiment $1 \mathrm{~B}$, subjects were good at remembering the twelve non-critical aspects of the movie, $M=9.42$ correct $(79 \%), S D=1.72$. To address the main research question-whether discussion with another person influenced subjects' memories-I analysed subjects' responses to discussed and nondiscussed details on the independent recognition test.

\section{Discussion Duration}

Before turning to the primary question, I first examined how long subjects took to complete the discussion. There was no evidence that people felt rushed to choose an answer during the discussion-if they did, the length of their discussions should have been close to the 12 minutes allowed. In fact no one took the full 12 minutes: subjects took between 2:43 min and 6:56 min to complete the discussion, $M=4: 37 \mathrm{~min}, S D=0: 59$.

Subjects were warned when only 10 seconds remained to answer a given question-this situation only arose 4 times out of a possible $240(2 \%)$. Importantly, for 3 of these 4 instances subjects were warned while discussing filler questions. Only one subject-pair was given a warning while discussing a critical question (out of a possible 80 instances). These results suggest that allowing subjects one minute to discuss each question retained control over the discussion phase of the study, yet did not limit subjects' ability to answer the questions, or force them to conform on critical details due to time pressure.

\footnotetext{
${ }^{3}$ Appendix J provides and explanation and justification for using the individual subjects-instead of the subject-pairs - as the unit of analysis.
} 
Not surprisingly, subjects did take slightly longer on average to discuss questions about critical details-where they had seen contradictory information-than questions about filler details. Subjects' discussions of critical questions ranged between $6 \mathrm{~s}$ and $61 \mathrm{~s}, M=25.4 \mathrm{o} \mathrm{s}, S D=7.12$, while their discussions of filler questions took between $7 \mathrm{~s}$ and $6 \mathrm{o} \mathrm{s}, M=21.88 \mathrm{~s}, S D=5.20, t$ $(19)=2.24, p=.02$, Cohen's $d=0.50$.

\section{Exposure to Misinformation}

Before I could turn to the primary research question-whether subjects influenced each other's memories-I needed to determine what the discussion partners told each other. In misinformation studies such as Experiment 1, subjects typically read or listen to a narrative containing misleading information (for example see Dodd \& Bradshaw, 1980; Echterhoff, Hirst, et al., 2005; Lindsay, 1990; Loftus, 1991; Loftus et al., 1978; McCloskey \& Zaragoza, 1985; Takarangi et al., 2006; Vornik et al., 2003); as a result, the experimenters have complete control of the information that subjects are exposed to. In my study, the method was not so simple; I relied on subjects to expose each other to the misleading information, and had no control over whether they did so. Instead, I could only examine what subjects did say after the fact; I used the experimenter's written record of subjects' discussion answers, and transcripts of the digital voice files to determine what was said during the discussion.

Subjects were exposed to between 1 and 4 misleading details, $M=2.03, S D=$ o.89; in total they were exposed to the misleading postevent information only 81 out of a possible 160 times $(51 \%)$. Because subjects were exposed to different numbers of misleading details, to compare subject's memories I calculated—for each subject—a proportion correct score for discussed and nondiscussed details. I use the word nondiscussed to refer to control details, the details that subjects were not asked about at all during the discussion. I use the word discussed to 
refer to the 81 instances in which subjects were exposed to the misinformation during discussion.

The remaining 79 instances-when subjects were asked about a critical detail during the discussion, but were not exposed to the misinformation-do not speak to my research questions, and were not included in any further analyses. This non-exposure occurred because subjects did not always assert the correct answer from their version of the movie during the discussion: sometimes they provided an incorrect answer either because they guessed or because they were mistaken and other times they simply agreed with what their partner said without offering an alternative.

\section{Remembering Discussed Details}

To answer the main research question-whether subjects were influenced by misinformation provided to them in a discussion-I compared the proportion of correct answers subjects gave for nondiscussed details with the proportion of correct answers they gave for discussed details. Did discussion affect subjects' memories of the critical details? As Figure 3.2 shows, the answer is yes. At test, subjects were more likely to report the correct answer for nondiscussed critical details than discussed critical details, $t(39)=6.82, p<.01$, Cohen's $d=1.11$. In other words, when subjects remembered discussed details, they sometimes reported what their partner told them instead of what they saw.

These results fit with the source monitoring explanation in Chapter 1: whether they were aware of it or not, subjects reported seeing details that they were only told about during the discussion. When people make source decisions, one factor that might be important is how they perceive the misinformation messenger. More specifically, people might make social appraisals about the messenger to decide whether the information conveyed is valuable or not (Johnson et al., 1993; Lindsay, 2008; Smith \& Ellsworth, 1987). For instance, as mentioned in Chapter 1, 
people tend to be misled when they judge the misinformation messenger to be credible, reliable, trustworthy, attractive or powerful, but resist the suggestions when they judge the messenger not to have those kinds of attributes (Dodd \& Bradshaw, 1980; Echterhoff, Hirst, et al., 2005; Vornik et al., 2003). In this study, misinformation was delivered to subjects via a peer who had ostensibly shared an experience with them. The results suggest that subjects generally judged others with whom they shared an experience to be a reliable source of information about that experience; as a result, they reported seeing details their partner only told them about.

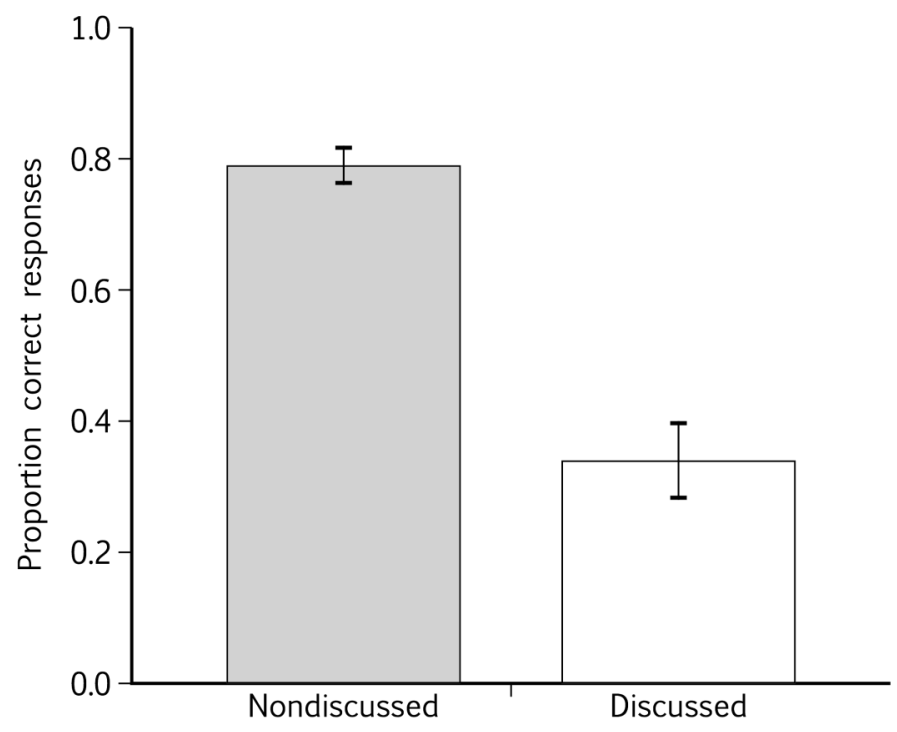

Figure 3.2. Proportion of correct responses for nondiscussed and discussed details. Error bars depict standard errors of the means.

I next examined the contribution of the eight individual critical details when they acted as nondiscussed details and when they acted as discussed details. These data appear in Figure 3.3. As the figure illustrates, subjects were more likely to give correct responses for the critical details when they had not been discussed, $t(7)=10.87, p<.01$, Cohen's $d=3.84$. 


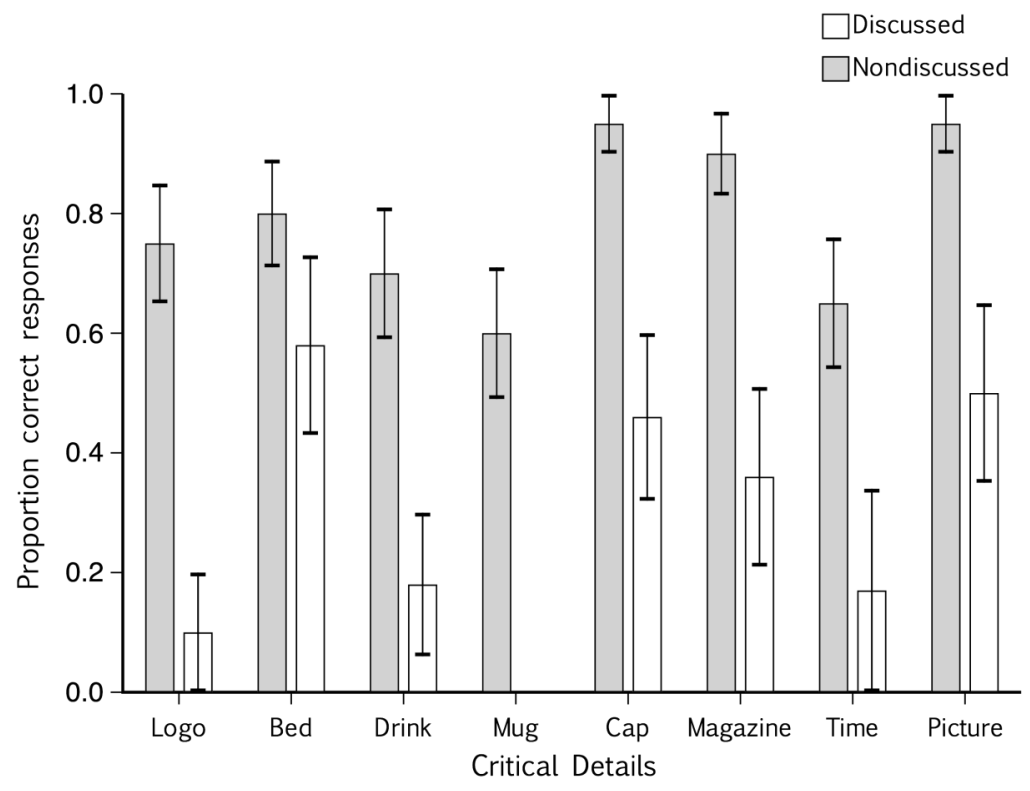

Figure 3.3. Proportion of correct nondiscussed and discussed responses for each critical detail. Error bars depict standard errors of the means.

In sum, both the subject-level analysis and the item-level analysis showed that discussion hurt subjects' memory performance; people were influenced by their partner's suggestions, and as a result were worse at remembering details they discussed. Importantly, people were not always misled by what their partner told them; instead, $33 \%$ of the time that people were exposed to the misinformation, they still gave the correct answer at test. There is some evidence that-like Asch’s $(1952 ; 1953 ; 1966)$ results—individual personality differences might have led subjects to be more or less influenced by their partner's suggestions. More specifically, when considering only subjects who were exposed to 2 or more pieces of misinformation, some subjects were misled by everything their partner told them (12 of $28 ; 43 \%$ ) yet others were never misled at all ( 2 of $28 ; 7 \%$ ); perhaps some kind of personality disposition might lead these subjects to respond in the same way across different details. For instance, Asch suggested that people's selfconfidence might determine how influenced they would be. In my study it might be that subjects who were never misled had high self-confidence, and subjects 
who were always misled had low self-confidence. The remaining 14 subjects (50\%) were misled on some details but not others; this result suggests that rather than making a blanket assessment of their discussion partner and everything their partner said, these people might have made source judgments based on different factors at different times. Put another way, different factors in the interaction might have led them to be more or less influenced by the misinformation.

Taken as a whole, these findings have implications for the way people remember the past. As discussed in Chapter 1, people talk about their past experiences all the time, and in doing so they probably expose each other to new information. These results show that when people talk about the past, they might incorporate new information from each other's reports into their own memory, and later report seeing something they did not see.

\section{Confidence}

As Figure 3.4 shows, and consistent with Experiment 1B, subjects were moderately confident in all their responses, and they were just as confident in their responses to discussed and nondiscussed details, $t<1$, ns. I next examined how confident subjects were in their correct and incorrect responses. As Figure 3.5 shows, relying on misinformation had a cost: when subjects claimed to have seen a critical detail in the movie, they were more confident about their correct memories for nondiscussed details than their false memories for discussed details, $t(32)=1.85, p=.04$, Cohen's $d=0.32$. Put another way, when subjects were misled by their partner's suggestions, they became less sure of themselves. 


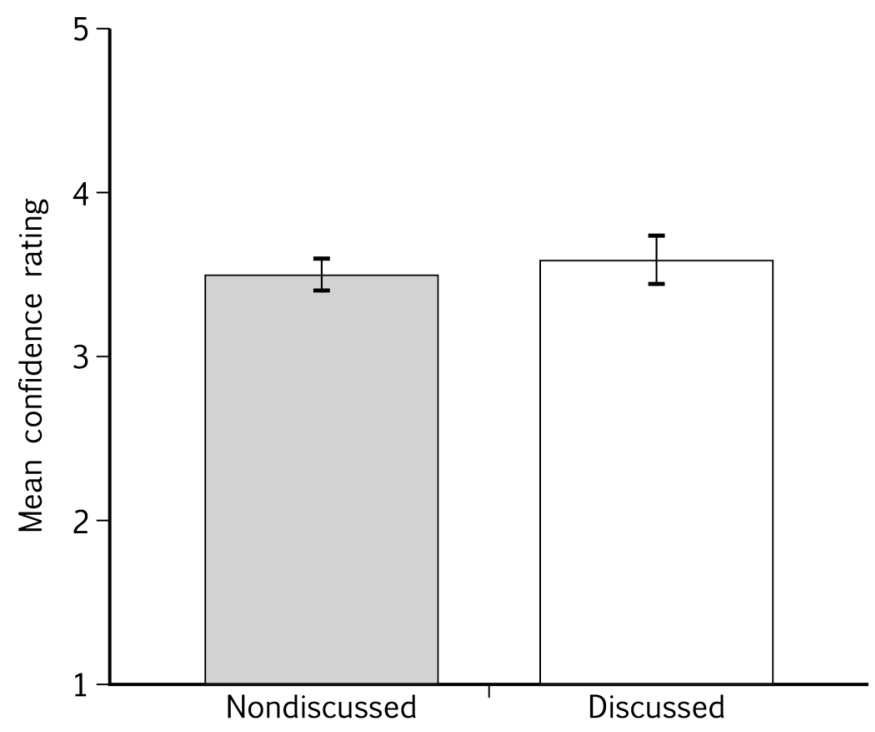

Figure 3.4. Mean confidence for nondiscussed and discussed details. Error bars depict standard errors of the means.

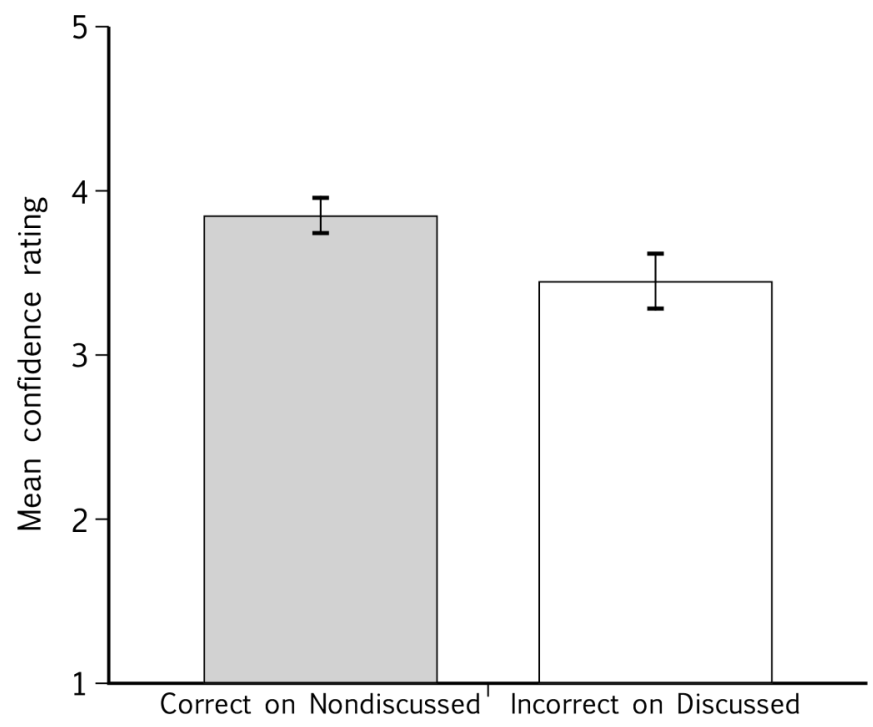

Figure 3.5. Mean confidence for correct responses on nondiscussed details (true memories) and incorrect responses on discussed details (false memories). Error bars depict standard errors of the means.

There are at least two explanations for this result. First, it might be that subjects were not $100 \%$ certain about what they recalled during the discussionthus when they did convince their partner to report the misinformation, at most 
they could only pass on their own less-than-perfect confidence. Second, it might be that the subject-pairs initially disagreed about the discussed details, and the confusion led them to doubt whatever their eventual answer was, thus decreasing their confidence.

Features of the Discussion

Even though subjects experienced decreased confidence they still reported seeing the misinformation. Most subjects were influenced by some of their partner's misleading suggestions but not by others; I wondered whether any features of the discussion led subjects to be more or less influenced by the misinformation. To address this question, I used transcripts of the discussions to examine four aspects of each subject's discussion for each critical detail: [1] if the subject spoke first; [2] if the subject disputed what his or her partner said; [3] if what the subject said was disputed by his or her partner; and [4] if the subject used the misleading detail as an answer to the critical discussion question. Interestingly, in every instance that one subject disputed what the other said during the discussion, the other subject reciprocated and disputed what the subject said. Whether coincidental, or the result of a conversational rule, the data for factors 2 and 3 matched perfectly, and there was no point in examining them separately. Instead, I combined the two factors to take into account whether or not there was a dispute in the discussion. I used the final three dichotomous discussion features, and examined the proportion of instances that subjects were and were not misled. Table 3.1 displays-for the 81 instances that subjects were exposed to the misinformation-the proportion of instances where each of the three discussion features occurred and the subsequent proportion of instances where subjects went on to be misled in the recognition test. As Table 3.1 shows, after subjects were exposed to the misinformation, they went on to use the misinformation (rather than what they personally saw) as their answer at test 
most—but not all—of the time.

Table 3.1

Proportion discussion feature occurrence and subsequent proportion subjects misled for 81 instances subjects exposed to misinformation

\begin{tabular}{lcc}
\hline \multicolumn{2}{c}{ DISCUSSION } & TEST \\
\hline Discussion Features & $\begin{array}{c}\text { Feature } \\
\text { (n cases) }\end{array}$ & $\begin{array}{c}\text { Misled } \\
\text { (n cases) }\end{array}$
\end{tabular}

Answer

Misinformation

$.73(59)$

$.85(50)$

Alternative

$.27(22)$

$.18(4)$

Dispute

Dispute

.36 (29)

No Dispute

$.64(52)$

$.88(46)$

Speaking Order

Spoke First

Spoke Second

$.78(63)$

$.76(48)$

OVERALL PERFORMANCE

$.67(54)$

Note. Proportion Correct $=1-$ Proportion Misled ( $\mathrm{n}$ cases Correct $=81-\mathrm{n}$ cases Misled).

Subjects always gave public responses during the discussion, and as such it is not possible to distinguish whether these responses were driven more by normative or informational influences (Deutsch \& Gerard, 1955). However, subjects' subsequent test responses do provide some insight. As displayed in the first two lines of Table 3.1, subjects used the misinformation as their final answer at test more often when they had previously used it as a discussion answer. Although these data do not provide a perfect measure, they suggest that most of 
the time that subjects agreed with their partner during the discussion, they did so because they believed their partner's recall to be accurate and reliable, and as a result went on to use that information as an answer at test. In other words they appeared to exhibit informational influence. However, some subjects also appeared to exhibit normative influence, complying with their partner and answering with the misinformation during discussion-perhaps for social reasons-but reverting to their own answer in the independent test (Deutsch \& Gerard, 1955).

More rarely, people sometimes answered with something other than the misinformation during the discussion, but went on to use the misinformation as their answer on the independent recognition test. There are at least two explanations for this finding. For instance, it might be that subjects resisted the misinformation during the discussion and instead answered with one of the filler alternatives. Then later, when the incorrect filler alternative was not offered on the final test, they shifted their decision to the misinformation. Alternatively, it might be that the influence of misinformation changed over time, leading people to be more influenced by it during the independent test than they were during the discussion. In fact, other research shows that misinformation can become more influential over time as it becomes less tightly tied to information about the misinformation messenger (Underwood \& Pezdek, 1998).

Table 3.1 also shows that subjects were misled less often when they disputed each other during discussion of the critical detail. However, sometimes when people disputed each other during the discussion, they were still eventually misled, suggesting that even if people initially resist or reject misinformation, they may still be influenced by it later on. Finally, only a small portion of the time that subjects were exposed to the misinformation did they speak first during the discussion of that detail. This finding suggests that speaking order 
may play some role in determining exposure to misinformation. Additionally, when people were exposed to the misinformation, they were misled less often when they spoke first.

In sum, there was no one pathway for misinformation: misinformation introduced to the discussion by another person was received in a variety of ways, with a number of different outcomes. Given the differences in how misinformation was received, I wondered if it was possible to predict whether someone would be misled on a given detail based on what happened during the discussion of that detail.

\section{Predicting False Memories}

Gabbert et al. (2006) found that whether people speak first about a given detail predicts whether they will be misled on that detail: the first speaker is much more likely to influence the second speaker's independent memory report than vice versa. One limitation to these findings is that the experimenters could not control whether subjects mentioned the misinformation to each other during discussion, and they did not account for exposure to misinformation in their analysis. As Lindsay (2007) observed, in Gabbert et al.'s study, sometimes when one subject spoke first, that subject was actually the only person to mention a detail at all; other times, the subject's discussion partner disputed his or her claim and mentioned the contradictory detail second. As a result, speaking first was confounded with exposure to misinformation: if someone spoke first about a given detail, and the other person did not respond, the second person was more likely to be influenced because the first person was never exposed to any misinformation.

I wanted to examine if any discussion features predicted whether subjects were misled on a particular detail in this study. More specifically I wanted to test whether my results fit with Gabbert et al. (2006), and I wanted to follow up 
Lindsay's (2007) concerns by investigating the role of exposure to misinformation in determining any predictors. To that end, I ran two nominal logistic regressions.

In both analyses, I treated critical details as independent cases, and entered the three dichotomous discussion features-whether the subject used the misinformation as a discussion answer, whether there was dispute during the discussion, and whether the subject spoke first-into the regression to predict if subjects were eventually misled on a particular detail. In the first regressionthe all-items regression - I followed Gabbert et al. (2006) and included all 16o instances where subjects were asked to discuss critical details, regardless of whether they were exposed to the misinformation when discussing those details. In the second analysis—-the exposure-only regression-I overcame Lindsay's criticism of Gabbert et al.'s analysis by including only the 81 instances that subjects were actually exposed to the misinformation during discussion. By considering only cases where subjects were exposed to the misinformation, everyone should have an equal chance of being misled on any given detail. Thus, I can rule out the possibility that any predictors are confounded with people not being exposed to misinformation, and thus being less likely (or unlikely) to be misled. In other words, in the exposure-only regression, if any features of the discussion do predict whether subjects are misled, it will show that those features are important in determining people's susceptibility to misinformation, while controlling for the possibility that those features are related to exposure to misinformation. If, as Lindsay (2007) suggested, speaking first only predicted being misled in Gabbert et al.'s study because exposure to misinformation was not taken into account, then speaking first should be a significant predictor of being misled in the all-items regression, but not in the exposure-only regression. All-items regression. When all 16o instances were included in the analysis, all 
three factors predicted whether subjects were misled on a particular detail. The odds ratios showed that subjects were 35 times more likely to be misled if they used the misinformation as an answer during the discussion, $\chi^{2}(1, N=160)=$ 29.30, $p<.01$, and they were 16 times less likely to be misled if there was dispute during the discussion, $\chi^{2}(1, N=160)=12.64, p<.01$. Additionally, in line with Gabbert et al.'s (2006) results, subjects were five times less likely to be misled if they spoke first when discussing a critical detail, $\chi^{2}(1, N=160)=10.32, p<.01$. Exposure-only regression. When considering only the 81 instances that subjects were exposed to misinformation, only two factors predicted whether subjects were misled. If subjects used the misinformation as their discussion answer, they were 21 times more likely to be misled, $\chi^{2}(1, N=81)=14.43, p<.01$, and if there was dispute during the discussion subjects were 17 times less likely to be misled, $\chi^{2}(1, N=81)=14.89, p<.01$.

Taken as a whole, these results point to three important conclusions. First, using the misinformation as a discussion answer was the best predictor of being misled later on. In both analyses, subjects were far and away more likely to be misled if they had previously used the misinformation as a discussion answer, suggesting a role for prior usage in influencing memory. Second, when subjects experienced dispute in the discussion they were much less likely to be influenced by the misinformation. Perhaps the experience of a shared dispute led subjects to be more deliberative, less trusting of what their partners said, and thus less likely to be misled. However, recall that in every instance where one subject disputed what the other subject said, the other subject reciprocated and disputed what the first subject said, so the two factors could only be analysed in combination. Rather than the experience of a reciprocal dispute, perhaps one of these features_-being disputed or doing the disputing — drives the effect. Finally, in 
support of Lindsay's (2007) suggestion, whether a subject spoke first when discussing a critical detail predicted being misled only in the all-items regression, where exposure to misinformation was not taken into account. In other words, the results suggest that the effect of speaking first might be driven by whether subjects were exposed to misinformation in the first place.

Speaking order may play an important role in the real world: speaking order might determine whether people will be exposed to misinformation, and thus whether they will be misled. To address this issue, I conducted another nominal logistic regression - the predicting-exposure regression - to see if any discussion features predicted whether subjects were exposed to misinformation.

Predicting-exposure regression. Both speaking order and dispute predicted whether subjects were exposed to the misinformation. Not surprisingly, when there was dispute in the discussion, subjects were 22 times more likely to be exposed to the misinformation than when there was no dispute, $\chi^{2}(1, N=160)=$ 23.42, $p<$.01. Additionally, the odds ratio showed that subjects who spoke first were 13 times less likely to be exposed to the misinformation than subjects who did not speak first, $\chi^{2}(1, N=160)=28.54, p<.01$. Even though speaking first might not be associated with susceptibility to false memories, these results support the idea that speaking first in a discussion is associated with whether people will come into contact with new information.

\section{Summary}

In sum, the revised materials from Experiment $1 \mathrm{~B}$ worked in combination with the MORI technique to produce a misinformation effect via discussion. Specifically, subjects experienced an event together, yet systematic discrepancies in their memory reports arose naturally in a discussion about the past, and led them to remember details of the event inaccurately. Subjects' discussions of the critical details were qualitatively different from one another and some of these 
differences predicted the outcomes for memory. Although the misinformation was received in a variety of ways, subjects' overall accuracy was worse for discussed than nondiscussed details: subjects sometimes reported seeing what their partner told them instead of what they saw. My next goal was to generalise this pattern of results beyond Takarangi et al.'s (2006) modified Eric event to ensure that the results were not tied to this specific stimulus set.

\section{Experiment 2B}

I created a new event to test the generalisability of the results in Experiment 2A. The new stimulus set was designed in the same way as Takarangi et al.'s (2006) materials, but featured a new target event, a party.

Method

\section{Subjects}

Forty IPRP students participated in pairs in return for course credit.

\section{Design}

The study had exactly the same design as Experiment $2 \mathrm{~A}$.

\section{Materials and Procedure}

The only differences between this study and Experiment $2 \mathrm{~A}$ were the materials used, and the filler task duration-everything else was identical. The misinformation effect in Experiment 2A was very strong (Cohen's $d=1.11$ ), and thus unlikely to be affected by other manipulations. In order to decrease the magnitude of the effect (so I would be able to examine the influence of different manipulations in subsequent studies) I shortened the length of the filler tasks to 10 min and 3 min respectively.

The new event was about a party-goer named “Chad.” Like Takarangi et al. (2006), I created two movie versions, with eight critical differences between them (see Table 3.2). For example, in one version of the movie Chad steals a wallet, and in the other he looks at the wallet but puts it back. 
Table 3.2

Critical differences between Chad movie versions

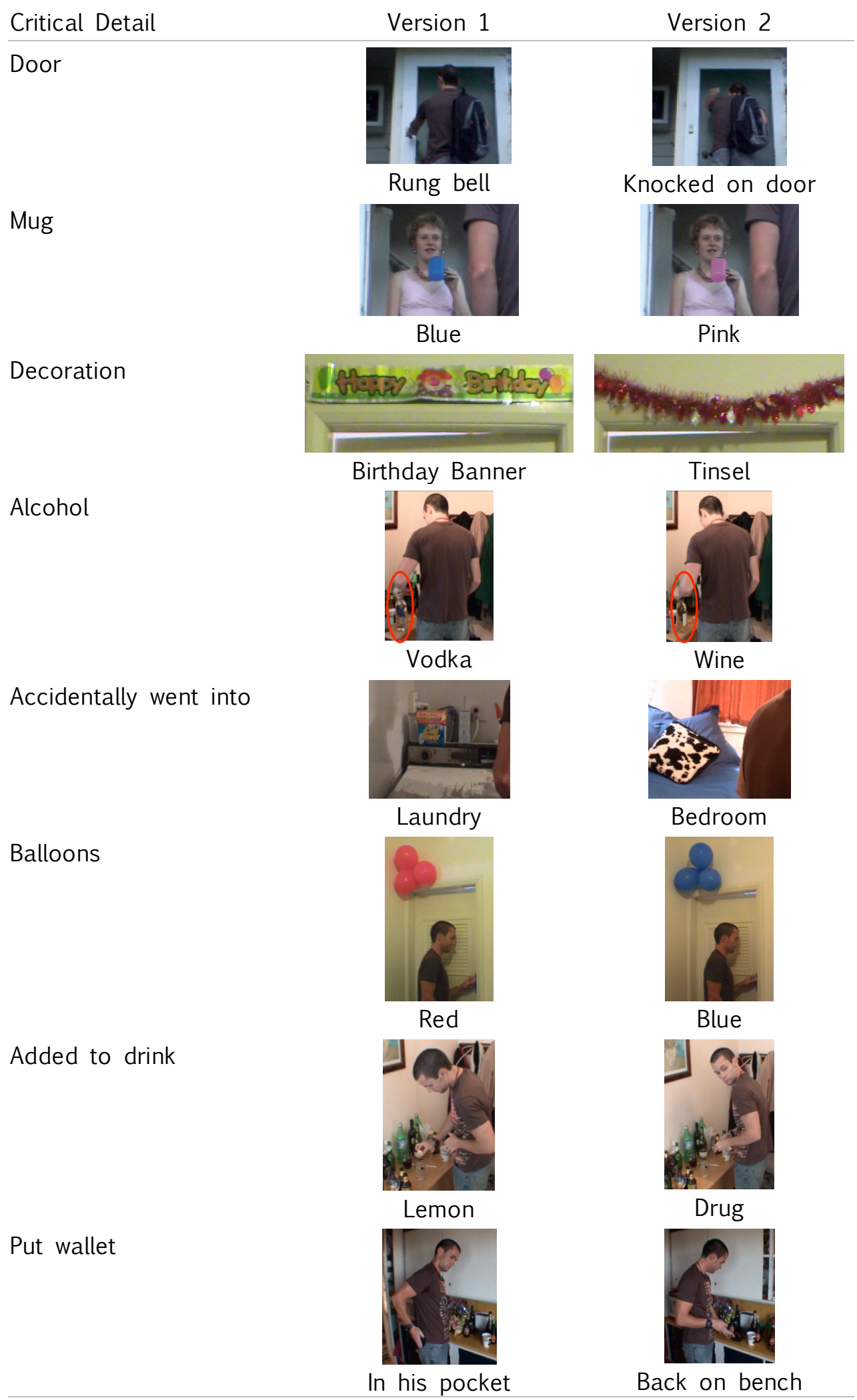


As in Experiment $2 \mathrm{~A}$, for each subject, four critical details acted as discussed details, and four acted as nondiscussed details. Critical details were counterbalanced so each served equally as a nondiscussed and discussed detail. The movie had no audio, and ran for 6:35 min. I updated the discussion slides and recognition test from Experiment $2 \mathrm{~A}$ to ask about details from the Chad movie. The structure of the questions, and the number of questions focused on critical and filler details was exactly the same as Experiment $2 \mathrm{~A}$.

Results and Discussion

All data were analysed on an individual level, yielding a total of 40 subjects. Consistent with Experiment 2A, subjects were good at remembering the twelve filler details, $M=9.08$ correct $(76 \%), S D=1.21$.

\section{Discussion Duration}

Also consistent with Experiment 2A, there was no evidence that subjects felt rushed to answer during discussion using the new stimulus set. Total discussion duration ranged from 2:45 $\mathrm{min}$ to 7:04 $\mathrm{min}$, well under the $12 \mathrm{~min}$ allowed, $M=$ 4:13 min, $S D=1: 12$. Additionally, only two times (out of a possible $80 ; 3 \%$ ) were subjects reminded that they had 10 seconds left when discussing critical questions. Five warnings (out of 160 possible instances; $3 \%$ ) were given when subjects were discussing filler details.

Subjects did take longer to discuss critical details than filler details. Discussion time for critical details ranged from 9 s to $59 \mathrm{~s}, M=24.10 \mathrm{~s}, S D=7.61$; discussion time for filler details ranged from 4 s to $72 \mathrm{~s}, M=19.53 \mathrm{~s}, S D=7.07, t(19)=2.37, p$ $=.01$, Cohen's $d=0.53$.

\section{Exposure to Misinformation}

Subjects were exposed to between o and 4 misleading details, $M=2.63, S D=$ o.98; in total they were exposed to misinformation on 106 out of 160 instances 
$(66 \%)$. As in Experiment $2 \mathrm{~A}$, I counted details where subjects were exposed to the misinformation during discussion as discussed details and details that were not asked about during discussion as nondiscussed details, and for each subject I calculated a proportion correct score for discussed and nondiscussed details. ${ }^{4}$ Remembering Discussed Details

Did discussion affect memory for the new movie? As Figure 3.6 shows, the answer is yes. Just like Experiment $2 \mathrm{~A}$, subjects were more likely to report the correct answer for nondiscussed details than discussed details, $t(39)=5.64, p<.01$, Cohen's $d=0.88$.

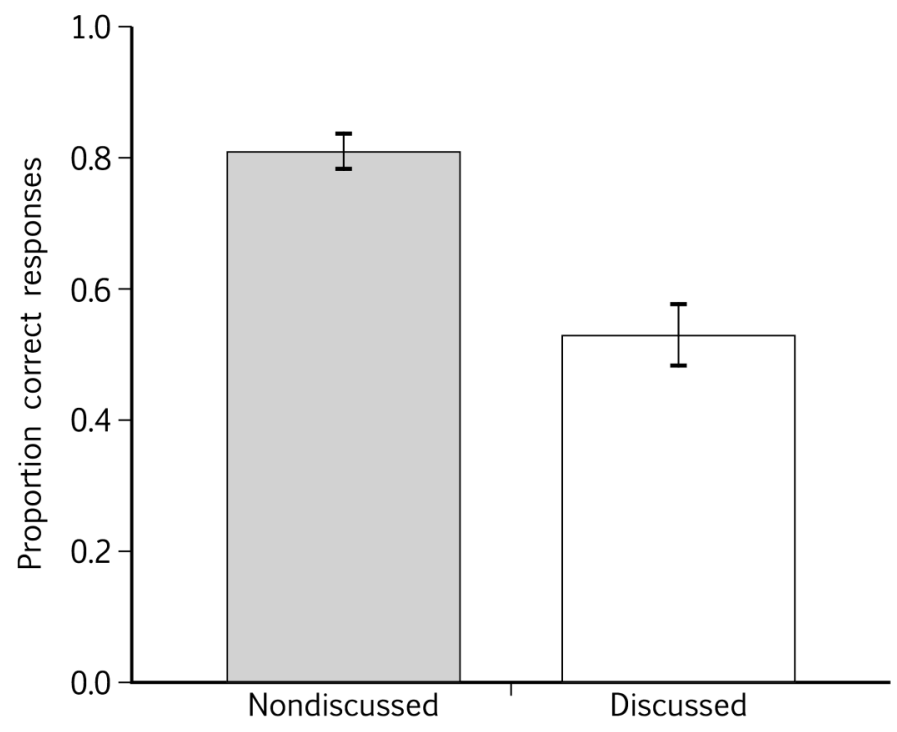

Figure 3.6. Proportion of correct responses for nondiscussed and discussed details. Error bars depict standard errors of the means.

Additionally, as displayed in Figure 3.7, analyses of each critical detail showed that subjects were more likely to give the correct response for critical details when they were not discussed than when they were discussed, $t(7)=3.89, p<.01$,

\footnotetext{
${ }^{4}$ Two subjects were exposed to misinformation for details that were intended to act as nondiscussed details; this situation seemed to arise when subjects tried to recall related details to help them answer a discussion question. For example, when trying to recall what colour the balloons were, one subject mentioned seeing red tinsel. These details were excluded from the analyses - the two subjects' proportion correct scores and related confidence judgments were based only on the three nondiscussed details that were not mentioned at all during the discussion.
} 
Cohen's $d=1.38$.

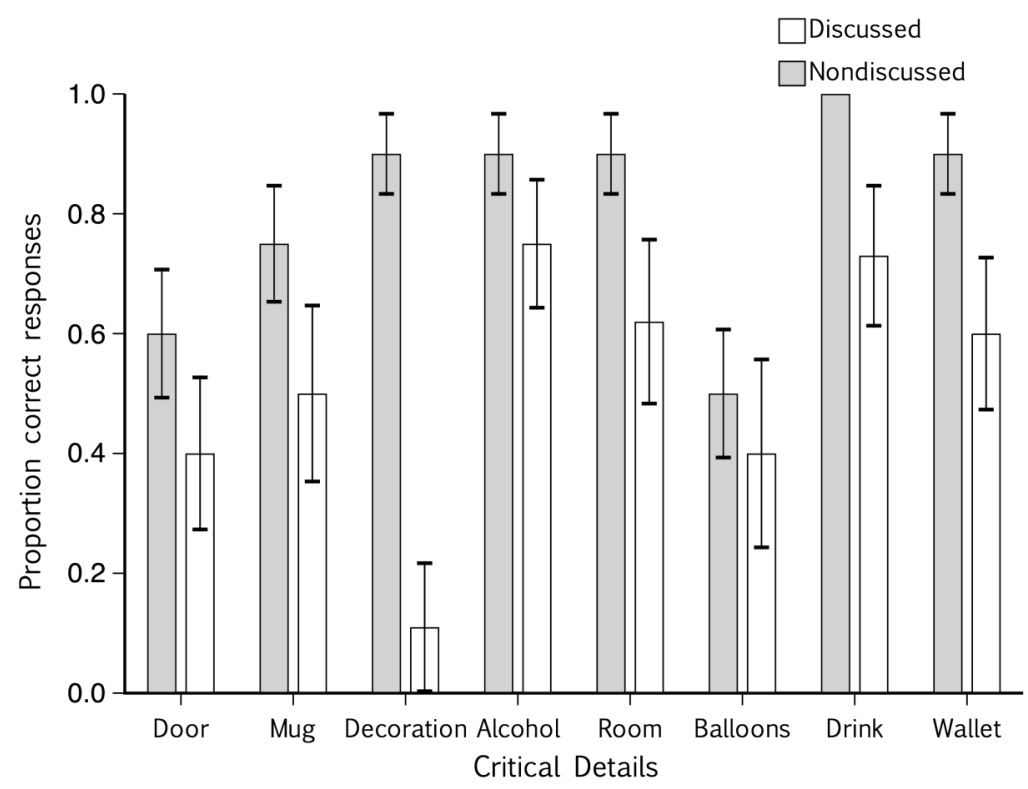

Figure 3.7. Proportion of correct nondiscussed and discussed responses for each critical detail. Error bars depict standard errors of the means.

Consistent with Experiment 2A subjects responded to the misinformation in different ways: considering only the 36 subjects who were exposed to at least two misleading details, some subjects were always influenced by what their partner said $(n=4 ; 11 \%)$, some were never influenced by what their partner said $(n=7$; $19 \%)$, and some were influenced some of the time $(n=25 ; 69 \%)$.

\section{Confidence}

Consistent with Experiment 2A, discussion did not affect subjects' confidence in their responses; as shown in Figure 3.8 subjects were moderately confident in their responses for both discussed and nondiscussed details, $t<1$, ns. Additionally, as shown in Figure 3.9, when subjects reported seeing a critical detail in the movie, they were more confident about their correct memories for nondiscussed details than their false memories for discussed details, $t(30)=3.32, p=<.01$, Cohen's $d=0.60$. 


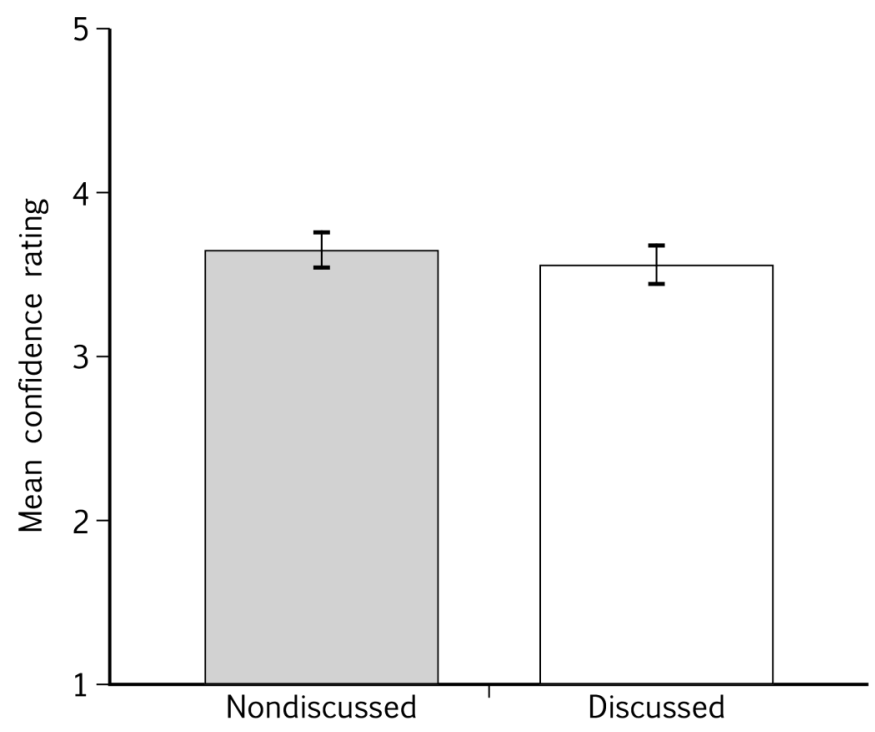

Figure 3.8. Mean confidence for nondiscussed and discussed details. Error bars depict standard errors of the means.

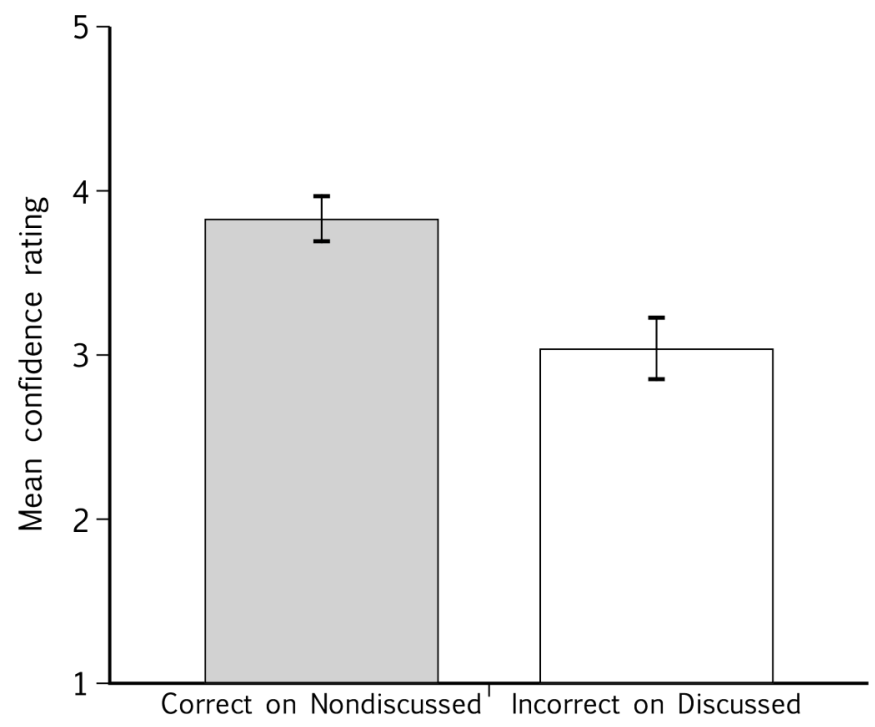

Figure 3.9. Mean confidence for correct responses on nondiscussed details (true memories) and incorrect responses on discussed details (false memories). Error bars depict standard errors of the means.

\section{Features of the Discussion}

As in Experiment 2A, I used transcripts of the subjects' voice files to determine what happened during the discussions. Of the 106 instances that subjects were 
exposed to misinformation, there were only eight instances in which subjects disputed what their partners said and their partners did not reciprocate. In other words, for the most part, data about whether subjects disputed their partners and whether subjects were disputed by their partners overlapped; the two factors were different enough that I could not combine them into one general item, yet not different enough to consider separately. I decided that whether subjects disputed what their partner said might shed more light on whether they were influenced by what their partner said so I included this feature in the analysis.

As in Experiment 2A, for each of the 106 instances where subjects were exposed to misinformation, I calculated the proportion of instances that each of the three discussion features occurred, and the subsequent proportion of times that subjects went on to be misled. These data are consistent with Experiment $2 \mathrm{~A}$ and are presented in Table 3.3. As the table shows, when subjects were exposed to misinformation, they used that misinformation as their answer at test nearly half of the time.

As displayed in the first two lines of Table 3. 3, subjects used the misinformation as their final answer at test more often when they had previously used it as a discussion answer; in other words, they appeared to exhibit informational influence. However, not all subjects behaved in this way; sometimes they appeared to exhibit normative influence, and used the misinformation as a discussion answer, but reverted to the correct answer on the test (Deutsch \& Gerard, 1955). Additionally, sometimes subjects answered with something other than the misinformation during the discussion, but used the misinformation as their answer on the independent recognition test.

Table 3.3 also shows that subjects were misled less often if they disputed what their partner said; however, people were sometimes misled by what their partner said even if they initially disputed it. Finally, in discussions where subjects were 
exposed to misinformation they rarely spoke first and they were misled less often when they did speak first.

Table $3 \cdot 3$

Proportion discussion feature occurrence and subsequent proportion of subjects misled for 106 instances that subjects were exposed to misinformation

\begin{tabular}{llc}
\hline \multicolumn{1}{c}{ DISCUSSION } & TEST \\
Discussion Features & $\begin{array}{c}\text { Feature } \\
\text { (n cases) }\end{array}$ & $\begin{array}{c}\text { Misled } \\
\text { (n cases) }\end{array}$ \\
\hline
\end{tabular}

Answer

Misinformation

$.58(62)$

$.74(46)$

Alternative

$.42(44)$

$.09(4)$

Dispute

Dispute

$.48(51)$

$.24(12)$

No Dispute

$.52(55)$

$.69(38)$

Speaking Order

Spoke First

.34 (36)

$.33(12)$

Spoke Second

$.66(70)$

.54 (38)

OVERALL PERFORMANCE

$.47(50)$

Note. Proportion Correct $=1-$ Proportion Misled ( $\mathrm{n}$ cases Correct $=106-\mathrm{n}$ cases Misled).

In sum, these patterns of results were very similar to those found in Experiment 2A: misinformation mentioned during the discussion was received in different ways at different times, and led to different outcomes.

Predicting False Memories

As in Experiment 2A, I next wanted to see if any discussion features predicted whether subjects would be misled on a particular detail. As such, I conducted the 
same two analyses, the all-items regression, and the exposure-only regression.

All-items regression. When including all instances that subjects were asked about critical details—regardless of whether they were exposed to misinformation - there were two predictors of whether subjects were misled. Subjects who agreed to use the misinformation as an answer during the discussion were 18 times more likely to be misled than those who did not, $\chi^{2}$ (1, $N=160)=37.74, p<.01$. Additionally, there was an interaction: subjects were three times less likely to be misled if they spoke first during the discussion of a critical detail, and they disputed what their partner said, $\chi^{2}(1, N=160)=4.39, p=.04$.

Exposure-only regression. Consistent with Experiment 2A, when including only the 106 instances that subjects were exposed to misinformation, whether subjects used the misleading information as an answer during discussion significantly predicted whether they would be misled. In fact, when subjects did use the misinformation as their discussion answer, they were 19 times more likely to be misled than when they used something else as their answer, $\chi^{2}(1, N=$ 106) $=21.94, p<.01$. Additionally, whether subjects disputed the accuracy of their partner's suggestions marginally predicted whether they would be misled, $\chi^{2}(1$, $N=106)=3.13, p=.08$; according to the odds ratio, subjects were less likely to be misled when they disputed what their partner said.

Taken together these results fit with Experiment 2A: Subjects were most likely to be misled if they used the misinformation as a discussion answer and were not as likely to be misled if they disputed what their partner said during the discussion. Importantly, whether subjects spoke first in discussing a particular detail only played a role in predicting being misled when exposure to misinformation was not considered.

Predicting-exposure regression. Consistent with Experiment 2A, speaking order and dispute both predicted whether subjects were exposed to 
misinformation when discussing a particular detail. According to the odds ratios, subjects were ten times more likely to be exposed to misinformation when they disputed what their partner said, $\chi^{2}(1, N=160)=15.90, p<.01$ and four times less likely to be exposed to misinformation when they spoke first, $\chi^{2}(1, N=160)=$ 6.90, $p<.01$. Additionally, there was an interaction between the two factors: when subjects spoke first but disputed what their partner said, they were three times less likely to be exposed to misinformation, $\chi^{2}(1, N=160)=5.22, p=.02$.

These results fit with those of Experiment $2 \mathrm{~A}$. As a matter of course, people cannot be misled unless they are exposed to misinformation (Lindsay, 2007). Speaking first plays a role in predicting exposure to misinformation, but when exposure to misinformation is taken into account, speaking first does not appear to predict susceptibility to false memories.

\section{Summary}

Taken together, these two experiments show that when people share an experience, and then talk about that experience together, they might incorporate details from each other's memory reports into their own memories. Although the misinformation was received in a variety of ways during the discussions, the net effect was to decrease subjects' accuracy when they later remembered discussed details independently. Specifically, subjects sometimes reported seeing details that contradicted what they actually saw. However, other times subjects resisted the influence of the misinformation. In both studies, subjects were more likely to be misled when they did not dispute what their partner said during the discussion and when they used the misinformation as their discussion answer.

Experiments $2 \mathrm{~A}$ and ${ }_{2} \mathrm{~B}$ demonstrate an effective method to research the influence of discussion on memory, across two different target events and stimulus sets. As described in Experiment 2A, using this method, subjects were able to experience an event together, yet systematic discrepancies in their 
memory reports arose naturally in a (structured) discussion about the past, and led them to remember details of the event inaccurately. The results of the two experiments suggest that people can incorporate details from other people's memories into their own memory when they discuss the past together.

One possible counterexplanation for these results—often raised by reviewers-is that the "blocked" movie version may not really be blocked at all. In other words, instead of being misled by what their partner told them, perhaps subjects saw both versions of the movie and were more likely to report the detail from the "blocked" version when their partner also mentioned that detail in discussion. If subjects did see both versions of a critical detail, I would expect them to say so during the discussion of that detail. Yet, subjects never mentioned seeing both versions of a detail, and often seemed surprised when their partner recalled a different detail. However, I cannot rule out the possibility that subjects did see both movie versions. My next goal was to address these concerns by establishing whether subjects could see the alternate version of the movie. 


\section{Chapter 4}

\section{Experiment 3: Noticing and Remembering the Alternate Movie}

Although the results of Experiments $2 \mathrm{~A}$ and $2 \mathrm{~B}$ suggest that people can come to remember seeing things that they only heard about during a discussion, reviewers often raise another possible explanation: perhaps subjects saw the alternate movie version-the version that was supposed to be blocked from subjects' view-thus the "new" information mentioned during discussion was not really new at all. In other words, perhaps people reported seeing the misleading details because they actually saw those details during presentation of the movie. However, there are several problems with this alternative explanation.

First, pilot testing with both naïve and informed subjects suggested that people could not see the alternate movie version. For illustration, Figure 4.1 displays photographs of how the laundry/bedroom critical detail appeared on the rear projection screen through the different polarising glasses. ${ }^{5}$ Second, subjects did not mention anything about seeing the other movie nor did they mention anything about seeing different versions of the critical details. If subjects saw Chad walk into a laundry and into a bedroom, they would have reported seeing both when trying to answer the relevant discussion question-yet no subjects did so. Third, subjects often seemed surprised when their partner recalled a contradictory detail during the discussion; if these subjects had also seen the contradictory details during the movie, they would not have been surprised by their partner's responses. Finally, if subjects did notice two versions of a critical detail they would have become suspicious about the study goals-perhaps even realising they were supposed to see something different from what their discussion partner saw. If subjects became suspicious about the study goals, it

\footnotetext{
${ }^{5}$ Because the light was projected from behind the screen-directly towards the camera-these photographs are not very clear. They are included simply to provide a rough idea of what subjects saw.
} 
would have increased their vigilance and source monitoring behaviour; it seems very unlikely that these people would be misled by anything their discussion partners told them. For instance, if subjects thought their discussion partner saw a different movie, they would have judged any information mentioned by their partner as unreliable, and thus should have resisted the influence of the misinformation (see Dodd \& Bradshaw, 1980, Echterhoff, Hirst, et al., 2005; Smith \& Ellsworth, 1987).

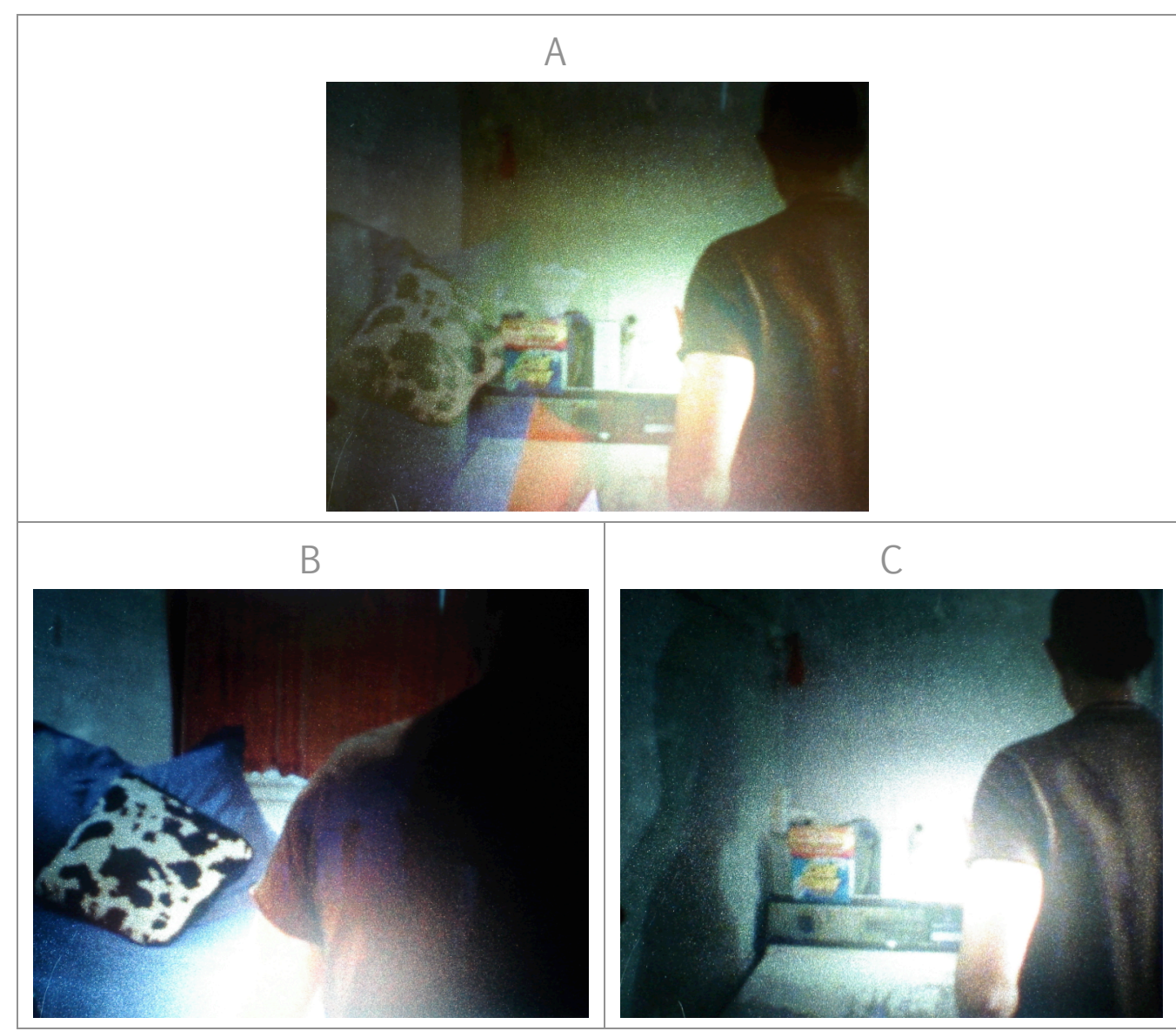

Figure 4.1. Overlapped laundry/bedroom critical scene photographed through no polarising filter (A); horizontal polarising filter (B); and vertical polarising filter $(\mathrm{C})$.

Despite these counterexplanations, in the studies reported so far I did not check if subjects noticed that there were two versions of the movie playing. Perhaps subjects did notice the alternate movie but—for some reason-they did 
not say anything about it. In some discussions, subjects did mention both versions of a critical detail; for example, subjects sometimes had difficulty deciding whether Chad knocked on the door or rang the doorbell. It seemed that these subjects were struggling to remember which one of the two actions they had seen, rather than struggling to decide which of two remembered actions they should answer with. However, there is no evidence to show that subjects did not see both. The usefulness of the MORI technique would be severely limited if subjects did notice that there were two versions of the movie and two versions of the critical details. As such, my next goal was to examine whether subjects noticed the alternate movie version while watching a movie via the MORI technique.

\section{Experiment 3A: Explicit Noticing}

One problem with simply asking people whether they noticed the alternate movie version is that they might report seeing the alternate version to save face rather than because they actually did notice it. I attempted to limit these kinds of response biases-where the questions demand a certain answer-in several ways.

First, rather than asking subjects straight out if they saw the alternate movie, I gave them a number of opportunities to give that answer in response to several less—but increasingly_suggestive questions. Second, to gather a baseline measure of how often subjects would respond "yes" to the critical questions, I used a between-subjects design. For half of the subjects, I played the two movie versions using the MORI technique, as in Experiments 1 and 2. For the other subjects, everything was set up identically but I played only one version of the movie. In other words, there was no alternate movie for these subjects to notice; if subjects in this condition reported seeing the alternate movie it would suggest that they did so due to the demand characteristics of the questions. Finally, I used Laney, Kaasa, Morris, Berkowitz, Bernstein \& Loftus' (2008) "Red Herring 
technique" to disguise the true purpose of the study.

\section{The Red Herring Technique}

In the Red Herring technique, researchers convey a fake cover story about the purpose of the study. Then, to stop the research-savvy subjects from deducing the true purpose of the study based on the questions they encounter, the critical questions are embedded among other questions that hint toward another fake

purpose. For example, as a cover story Laney et al. (2008) told subjects they were interested in how personality was related to food preferences; they were really interested in whether they could change people's eating preferences by leading them to falsely believe that they had either a good or bad experience with asparagus during childhood. To disguise the true purpose of the study, Laney et al. hid the critical asparagus questions among questions that implied they were interested in the problem of obesity.

In this study, I used the same cover story as Experiments 1 and 2-that I was interested in sensory impressions at different levels of visual acuity-in combination with the Red Herring technique. I disguised the critical questions about noticing the alternate movie version among questions that asked subjects to make social appraisals of the main character, Chad.

If subjects reported seeing the alternate movie version, and especially if they did so in response to the least suggestive questions, it would support reviewers' concerns that people might see the "blocked" movie version when watching a movie via the MORI technique.

Method

\section{Subjects}

In total forty subjects participated in pairs; 14 were IPRP students who took part in return for course credit, and 26 were recruited by advertising around the campus and received a $\$ 10$ grocery voucher. 
Design

The experiment was a between-subjects design. Half of the subjects made up the overlap group; they watched one version of the Chad movie while the alternate version was played and blocked via the MORI technique. The remaining subjects made up the non-overlap group; these subjects also watched one version of the Chad movie, but the alternate movie version was not played. Materials and Procedure

Aside from whether subjects were in the overlap or non-overlap group, the setup of the experiment was exactly the same as Experiment ${ }_{2} \mathrm{~B}$. However, there was no memory test in this study; immediately after watching the Chad movie, subjects worked through a question booklet independently.

Question booklet. The question booklet contained 13 questions. After answering each question, subjects were asked to provide an explanation for the answer that they gave. Eight of the thirteen questions were Red Herring questions, and implied that I was interested in subjects' social judgments about Chad. For example, one Red Herring question read, "If you saw Chad at the party, would you have tried to change his behaviour in any way?"

The four critical questions appeared in positions 5, 10, 11 and 12 and increased in suggestiveness as subjects progressed through the booklet. The first critical question asked subjects if they noticed anything unusual about the movie. Next subjects listed all the reasons they could think of that they might not agree with some of their partner's answers if they were asked to discuss the movie together. Then, to test the Red Herring, the third question asked subjects what they thought the true purpose of the study was. The last critical question asked subjects whether they thought they saw the same movie as the person next to them. Finally, question 13 asked subjects what they knew about the study before they took part. Subjects were asked to work through the booklet in order, and not 
to return to any previous pages.

Results and Discussion

To answer the overarching question-whether subjects noticed the alternate movie version-I address each of the four critical questions in turn.

Did subjects notice anything unusual about the movie? In fact, every subject in the non-overlap group and 18 subjects $(90 \%)$ in the overlap group reported noticing something unusual about the movie. I examined subjects' explanations to see what was unusual. In the vast majority of these explanations subjects criticised the quality of the movie and the lack of sound (evidently my movie making skills were not quite up to the discerning subjects' expected standards).

One subject from the overlap group did mention seeing multiple images; her explanation read "Chad's actions kind of doubled like when you have bad reception on $T V$ and you see 2 people rather than one." The angle of this subject's head and/or polarisation glasses must have changed the angle of the polarisation allowing her to see parts of both movie versions. However, she did not mention seeing any conflicting details, just poor quality images.

Additionally, this subject did not conclude that the double images resulted from different movies; instead, in the subsequent questions she made no mention of the two images. When listing all the reasons that she may not have agreed with her partner if they discussed the movie together, she did not even entertain the possibility that they might have seen different movies. Most interesting though, when this subject was asked at the end of the study whether she thought she had seen the same movie as her partner, she replied "yes" and gave the explanation "There's one screen. How can there be two movies playing?"

In sum, the vast majority of subjects did not notice the alternate movie and the one subject who did report seeing two images seemed not to even consider the possibility that there were two movies playing. 
Reasons for disagreement. The next critical question asked subjects to list all the reasons that they might not agree with the person next to them if they discussed the movie. Subjects listed between o and 10 reasons, $M=3.65, S D=1.81$; the majority centered on people having different backgrounds and experiences, and seeing the world differently depending on their personality, opinions, beliefs or expectations. Additionally, a number of subjects mentioned the possibility that they may have paid attention to different things in the movie, or they may have had different eyesight ability to begin with.

Three subjects mentioned the possibility that they may have seen something different from their partner. All three of these subjects were in the non-overlap group, suggesting that their ideas were speculative, rather than based on noticing the alternate movie version. Additionally, all three subjects provided between one and four other reasons why they might have disagreed with their partner suggesting that they were simply noting a possibility, rather than genuinely believing that they did see something different. In short, when confronted with the situation that subjects in Experiments $2 \mathrm{~A}$ and $2 \mathrm{~B}$ experienced, most people did not even consider the chance that they might have seen something different from their partner.

True purpose of the study. The Red Herring was effective at hinting a fake reason for the study: the majority $(83 \%)$ of subjects' responses were broadly consistent with the Red Herring theme and focused on judgments of behaviour. Not one subject mentioned anything to do with multiple images in response to this question.

Did subjects think they had seen a different movie to their partner? The vast majority $(93 \%)$ of subjects thought they had seen the same movie as their partner, and many subjects expressed surprise at the question. For example, subjects' explanations included: 
Subject Ka: We both looked at the same screen... therefore the same movie? And I would be totally surprised at how you could get us to look at the same screen yet see different things...

Subject Pa: $\quad$ There was only one screen and we were both looking at it together, I can't imagine how we both could have watched different movies simultaneously on the same screen...

Subject $\mathrm{Rb}$ : We were in the same room at the same time watching the same movie-I don't think the glasses were going to change that.

Subject $\mathrm{Db}$ : He is sitting about half a metre away from me, looking in the same direction. I would like to think if there were another screen I would have been able to see it.

However, three subjects were not so sure. Two subjects did not think they had seen the same movie as their partner, and another one provided an ambiguous response. One subject who thought he had seen a different movie to his partner was in the non-overlap condition, so could not have seen the alternate movie. He deduced that there were two movies because the earlier questions implied that there were:

Subject Da: Because the questions before that on "if you two were asked about the movie do you think you may disagree on things?” seemed to imply that we would disagree on things because we did in fact watch different movies.

The other subject who thought she saw a different movie from her partner was in the overlap group. Yet, rather than believing there were different movies, her explanation implied that she thought people would experience the same movie differently depending on their own histories.

Subject Ga: We both would have assumed Chad to be doing different things depending on our past experiences. 
Finally, another subject in the overlap condition gave an ambiguous response; again, there was no evidence that this subject noticed the alternate movie version. His explanation read:

Subject Fa: I sort of thought maybe we were seeing different movies we laughed at different times. Might just be because we thought those bits were funny, but I don't really know.

\section{Summary}

Taken together, these results show that for the most part, subjects did not notice the alternate movie version when they watched a movie via the MORI technique. Additionally, subjects were not particularly suspicious that I might have shown them different movies-despite the demand of the critical questions, the one subject who did notice overlapping images, did not think the different images came from different movies. Considering these results in combination with the fact that subjects in Experiments $2 \mathrm{~A}$ and ${ }_{2} \mathrm{~B}$ did not mention seeing conflicting versions of the critical details, it seems fair to conclude that generally, subjects who watch a movie via the MORI technique, do not notice the alternate movie or the alternate versions of the critical details.

However there is another way that using the MORI technique might promote a misinformation effect. Perhaps-even though subjects were not aware of it and did not explicitly report it - they saw the conflicting details without realising and somehow their implicit memory of the alternate movie influenced their discussion behaviour or their responses at test. For instance, perhaps subjects saw both movie versions and were simply more likely to report the details from the alternate version when their partner also mentioned those details during discussion. My next goal was to test the possibility that subjects had implicit memories of the alternate movie. 


\section{Experiment ${ }_{3} B$ : Implicit Memory}

To investigate whether subjects had any implicit memory of the alternate movie version, I used a "second-choice test." In a second-choice memory test, each question asks about a given detail and provides a list of alternative answers for subjects to choose from. After answering the question, subjects are asked to make a second choice, and pick their next best answer from the remaining options on the list. If subjects in my studies remembered aspects from both the movie version they saw and the alternate movie version, it should show up in their second choices. For instance, if Mollie remembered aspects of Chad in a laundry and aspects of Chad in a bedroom, she should use one of these answers as a first response and the other as a second response, thus demonstrating that aspects of both movie versions were accessible in her memory. One caveat is that subjects might also report both versions of a critical detail because the two options seem the most likely two of the offered alternatives. To control for the possibility that subjects would choose the correct detail from the alternate movie without any memory of it, I compared responses for overlapped details with responses for non-overlapped details, details for which subjects could not have any memory of the alternate movie.

If the MORI technique led subjects to have implicit memories of the alternate movie their responses might be affected in several ways. First, their implicit memory of the alternate version might work to change the way they remember and report critical details from their own version. If subjects' ability to report the correct critical details was affected by the alternate movie version, they would be worse at remembering overlapped details, and less confident when remembering overlapped details, relative to non-overlapped details. Second, if subjects did have some memory of the alternate version, they should be more likely to pick the correct answer from that version for overlapped than non-overlapped details. If 
subjects are poorer at remembering overlapped details, less confident when remembering overlapped details or better at choosing the answer from the alternate movie version for overlapped details, it would provide evidence that people's memories are influenced by the alternate version when movies are presented via the MORI technique.

\section{Method}

\section{Subjects}

In total, 40 students from the IPRP pool took part in the study in return for course credit. Each session had either one or two subjects.

\section{Design}

I used a within-subjects design: subjects saw one version of the Chad movie with half of the critical details overlapped with the alternate movie version (blocked via the MORI technique) and half of the details not overlapped with any image.

\section{Materials and Procedure}

Movie. The experiment was set up in the same way as Experiment $3 \mathrm{~A}$, except I used four new versions of the Chad movie in combination with the original two versions. To create the four new versions, I split each of the original versions into two new half-length movies; each new half-version included four of the eight critical details. I used iMovie ${ }^{\circledR}$ to fill up the remaining duration of the new movies with a blank image; the half length movies kept time with the full length movies, but nothing was projected onto the screen for the blank half (see Table 4.1). In the first-half movie versions, the Chad movie played for 3:03 min and then faded into a blank image for the remaining 3:31 min; for the second-half movies, a blank image played for 3:03 min and then faded into the Chad movie, which played for the remaining 3:31 min. 
Table 4.1

Critical details in each of the four half-length Chad movie versions

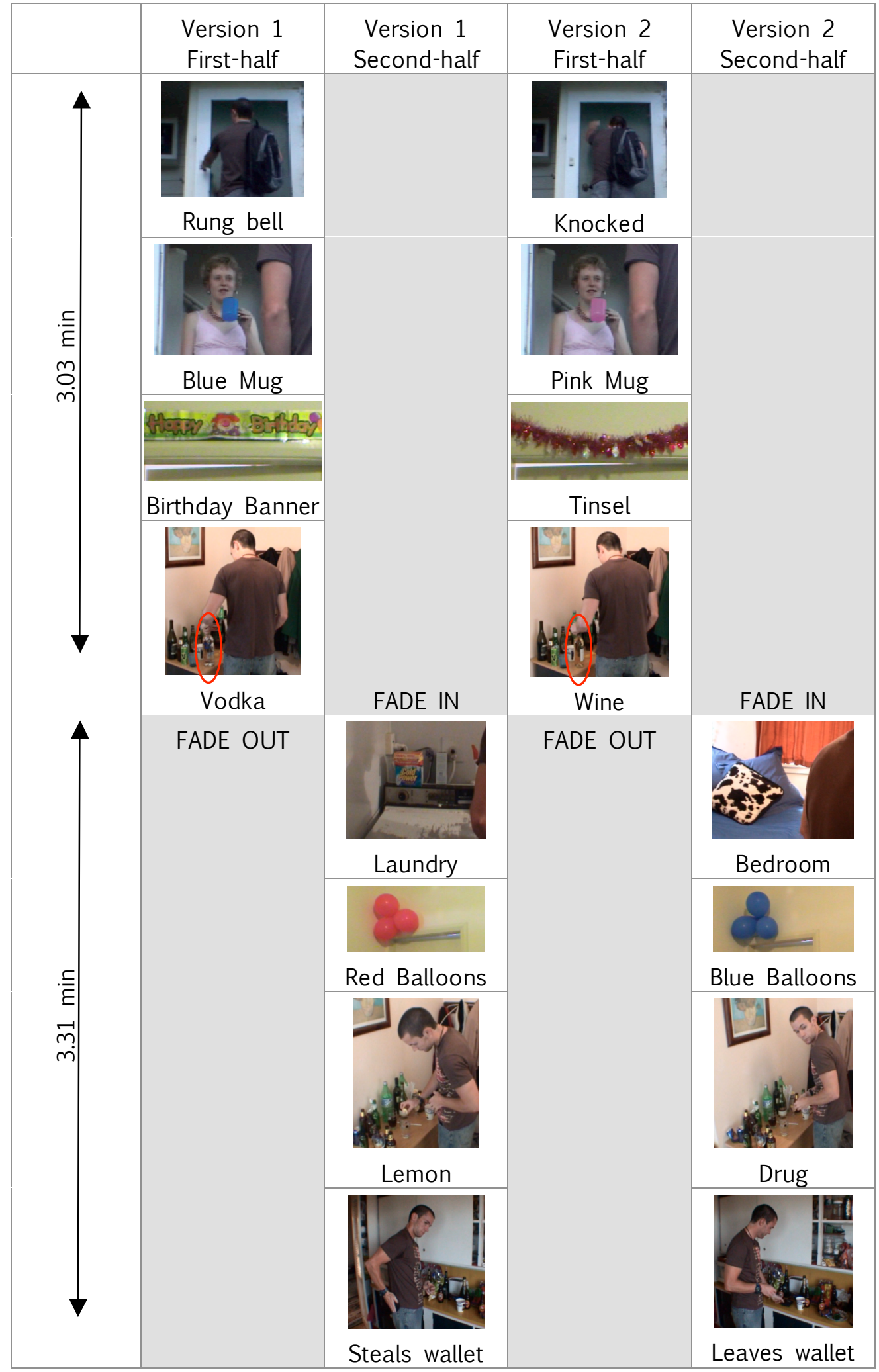


For example, a subject might have been shown Version 1 of the movie overlapped with the new Version 2 first-half movie. In this case, the first four critical details in the movie would have acted as overlapped details. After these details were presented (3:03 min through the movie) the Version 2 first-half movie faded to a blank image, and the remaining four critical details would have acted as non-overlapped details. Critical details were fully counterbalanced and acted equally often as overlapped and non-overlapped details.

In order to parallel the time subjects in Experiments $2 \mathrm{~A}$ and ${ }_{2} \mathrm{~B}$ took to complete the filler tasks and discussion (between the movie and the recognition test), following the movie subjects completed filler tasks for 20 minutes. After the filler tasks, subjects completed a second-choice recognition test.

Test. There were twelve questions on the test-eight related to the eight critical details and four were fillers. Each question asked about a detail from the movie and suggested five answers. For the critical questions, the correct answer from each movie version was listed among the five alternatives. For example, one question read "Where did Chad put the wallet that he found on the bench? In the cupboard; Behind the microwave; Back where he found it; In his back pocket; In his bag." Subjects selected their answer and rated their confidence on a 5-point scale, where $1=$ not at all confident and $5=$ very confident. Subjects were then asked to answer the same question again, providing their next best response. Finally, subjects were asked to explain why they selected their second response.

Results and Discussion

Unlike Experiments 1 and 2, subjects' performance on the four filler questions was quite poor, $M=2.25$ correct $(56 \%), S D=0.81$. In retrospect, this result is not too surprising; two of the filler questions were originally discussion questions designed to encourage discussion between subject-pairs, and as such were quite difficult to answer. 


\section{Remembering Overlapped Details}

I first calculated the proportion of correct responses subjects gave for overlapped and non-overlapped critical details; these proportions appear in Figure 4.2. As the figure illustrates, subjects' initial responses were just as likely to be correct whether the images were overlapped or not, $t<1, n s$. Additionally the confidence interval-for the difference between nonoverlapped and overlapped details-was narrow, suggesting the null results were not due to a lack of power, $95 \% C I=-0.12<\mu 1-\mu 2<0.08$ (Aberson, 2002; Loftus, G., 1996). Finally, the effect size and corresponding confidence interval were also small, providing more support for the null result, Cohen's $d=.09,95 \% C I=.01<\mathrm{D}<.15$ (Aberson, 2002; Thompson, 2002) ${ }^{6}$. Taken together, these results suggest that presenting a movie via the MORI technique did not affect subjects' memories of the critical details from their version of the movie, or their ability to report those memories.

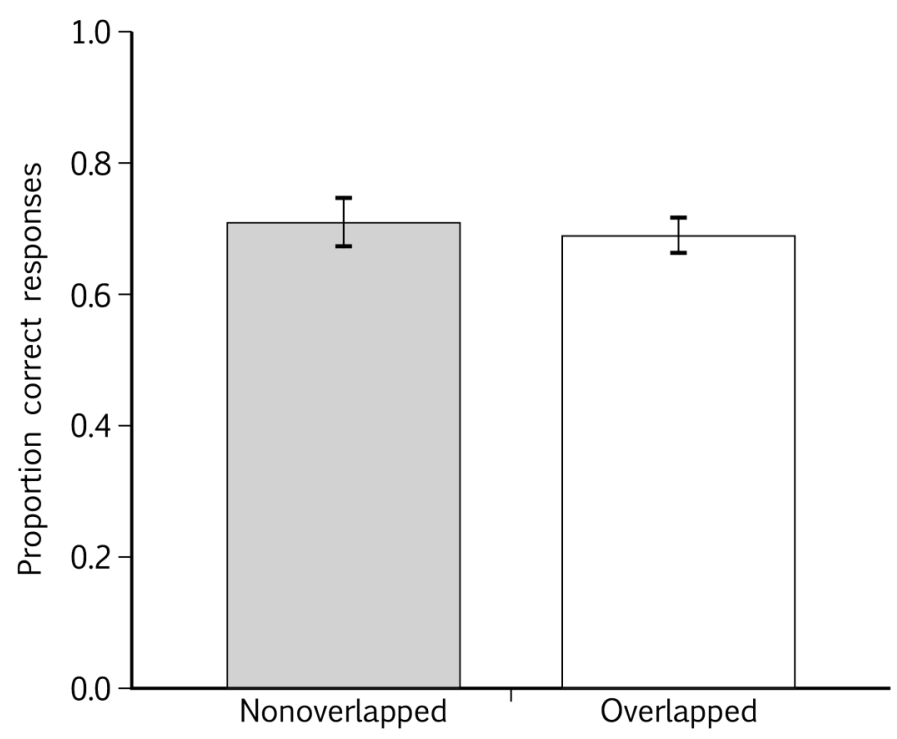

Figure 4.2. Proportion of correct responses for nonoverlapped and overlapped details. Error bars depict standard errors of the means.

${ }^{6}$ Confidence intervals for the effect sizes were obtained using the calculator available at http://wilderdom.com/301/tutorials/Tutorial7-Power.html 
Although subjects' overall memory accuracy was not affected by the overlapped images, their memory for the individual critical details might have been. I next examined subjects' responses for each of the eight critical details. These data are displayed in Figure 4.3. As the figure illustrates, subjects gave similar responses for the critical details whether they were overlapped or not, $t<1$, ns.

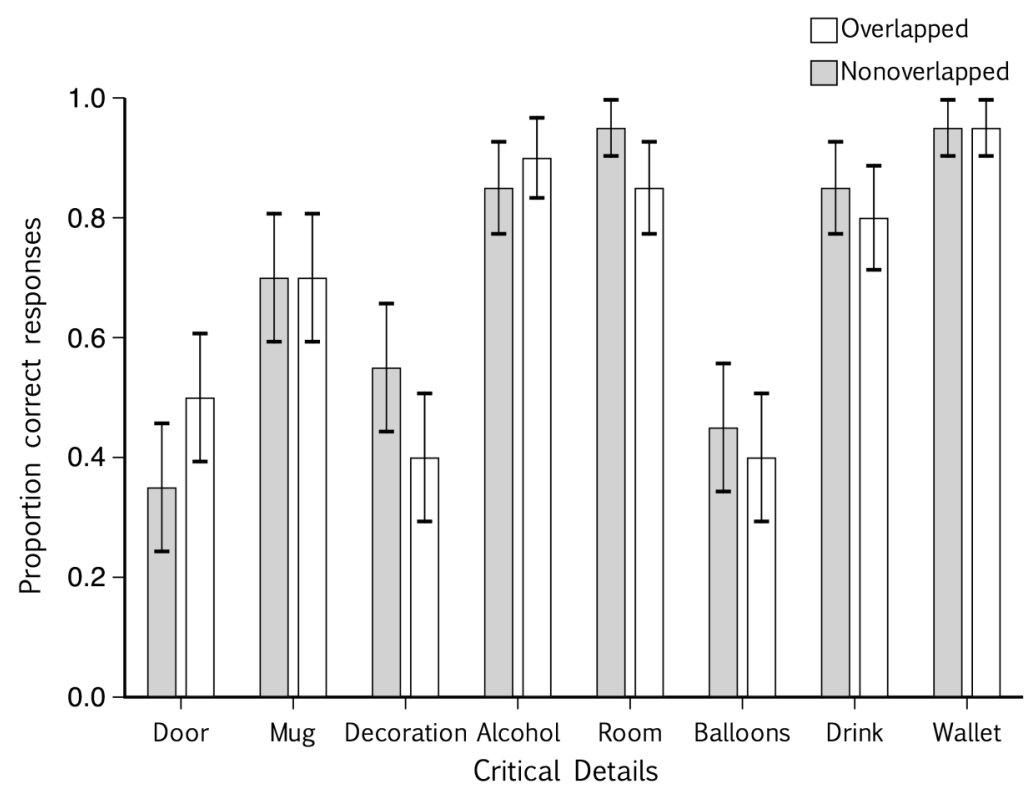

Figure 4.3. Proportion of correct nonoverlapped and overlapped responses for each critical detail. Error bars depict standard errors of the means.

\section{Confidence}

Figure 4.4 illustrates that subjects were just as confident in their answers for overlapped and non-overlapped details, $t<1, n s$. Additionally, the confidence interval for the difference was small, $95 \% C I=-0.28<\mu 1-\mu 2<0.35$, as was the corresponding effect size, Cohen's $d=.05,95 \% C I=-0.18<\mathrm{D}<0.25$, providing more support for the null result. Taken together, these results suggest that the alternate movie version did not affect the subjective feelings associated with remembering critical details. In short, subjects were just as good at remembering overlapped and non-overlapped details, and just as confident at remembering 
overlapped and non-overlapped details. These results suggest that the MORI technique does not hurt subjects' memory of the critical details.

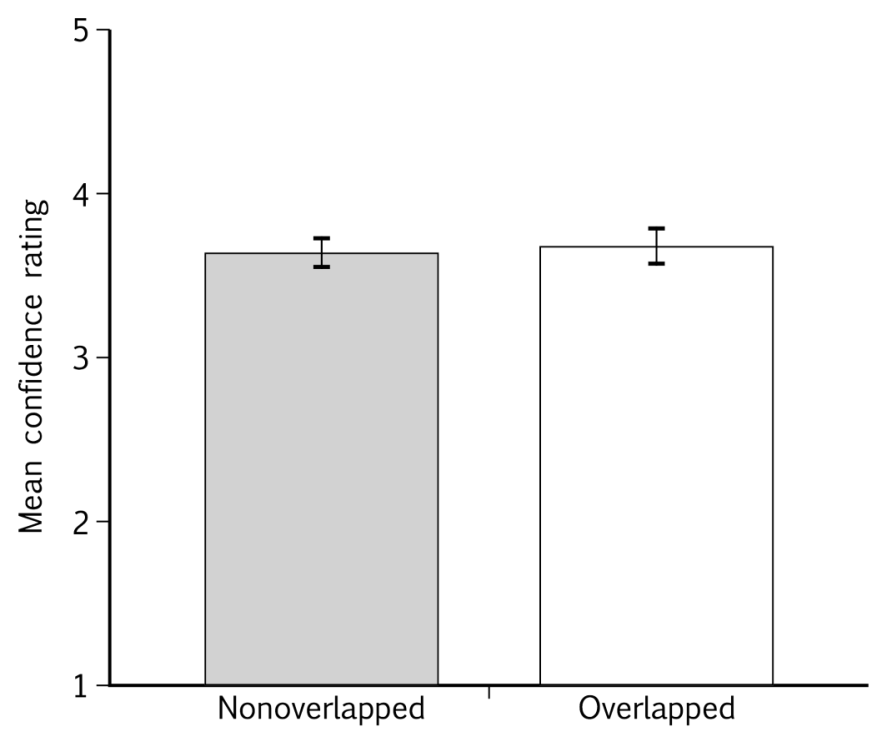

Figure 4.4. Mean confidence for nonoverlapped and overlapped details. Error bars depict standard errors of the means.

\section{Remembering Details from the Alternate Movie Version}

Next, I calculated the proportion of subjects' first and second responses where they gave the correct answer from the alternate movie version. I compared these proportions for overlapped and non-overlapped details; these data are displayed in Figure 4.5. As the figure shows, subjects did not demonstrate any memory of the critical details from the alternate movie version. Subjects were equally likely to pick the correct answer from the alternate movie version for overlapped and non-overlapped details for both their first responses and their second responses, ts $<1$, ns. Additionally, the confidence intervals around the differences were narrow, suggesting that the results were not due to a lack of power, $95 \% C I=-$ $0.09<\mu_{1}-\mu_{2}<0.07$ and $95 \% C I=-0.14<\mu_{1}-\mu_{2}<0.06$, respectively. The effect size (for the difference between nonoverlapped and overlapped details) for subjects' first choices was small, Cohen's $d=.04,95 \% C I=-0.02<\mathrm{D}<0.09$, further 
supporting the null result. However, there was a more substantial effect size for subjects' second choices, Cohen's $d=.18,95 \% C I=0.11<\mathrm{D}<0.25$. Importantly, as displayed on Figure 4.5, any difference for subjects' second choices is in the opposite direction to what reviewers are concerned about. In other words, even if there is an effect, it is for subjects to be slightly better at selecting the correct answer for the alternate version for nonoverlapped than overlapped details. Taken together these results suggest that subjects do not have any memory of the alternate movie version.

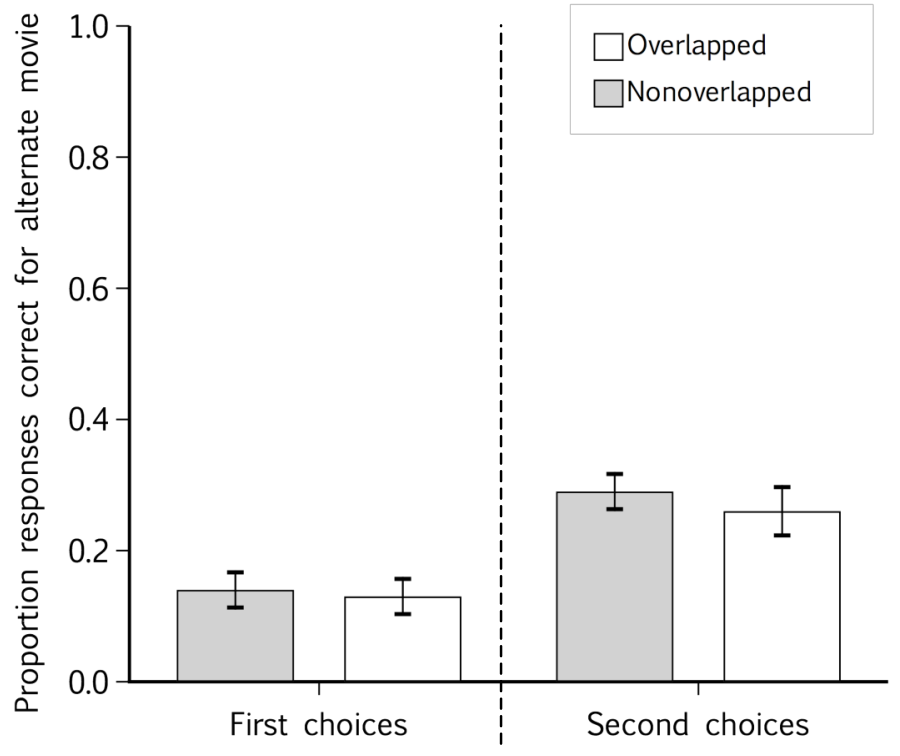

Figure 4.5. Proportion of first and second choices correct for alternate movie version for nonoverlapped and overlapped details. Error bars depict standard errors of the means.

Although overall subjects were no more likely to pick the correct answer from the alternative movie version for nonoverlapped or overlapped details, they may have been more likely to do so for some specific critical details. I next examined the proportion of instances where subjects chose the correct answer from the alternate movie version for each critical detail when it acted as a nonoverlapped detail and an overlapped detail. Figure 4.6 shows that there were no differences for subjects' first choices, and Figure 4.7 shows there were no differences for 
subjects' second choices, both $t \mathrm{~s}<1, n s$. In short, subjects were no more likely to pick the correct answer from the alternate movie version when those details were overlapped.

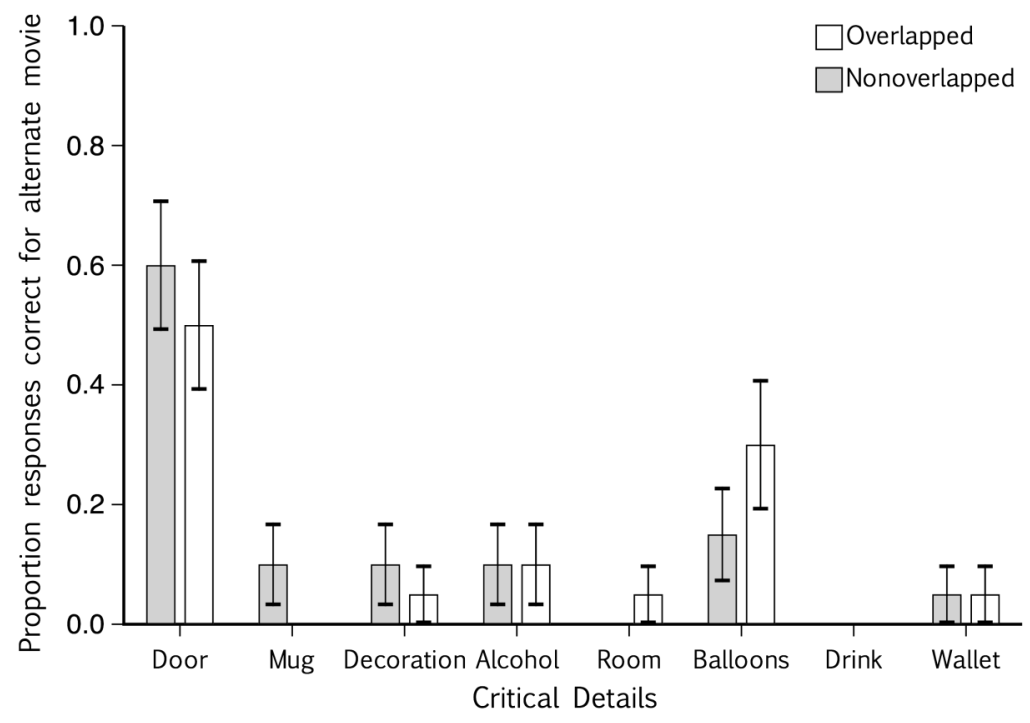

Figure 4.6. Proportion of first choices correct for alternate movie version for nonoverlapped and overlapped details. Error bars depict standard errors of the means.

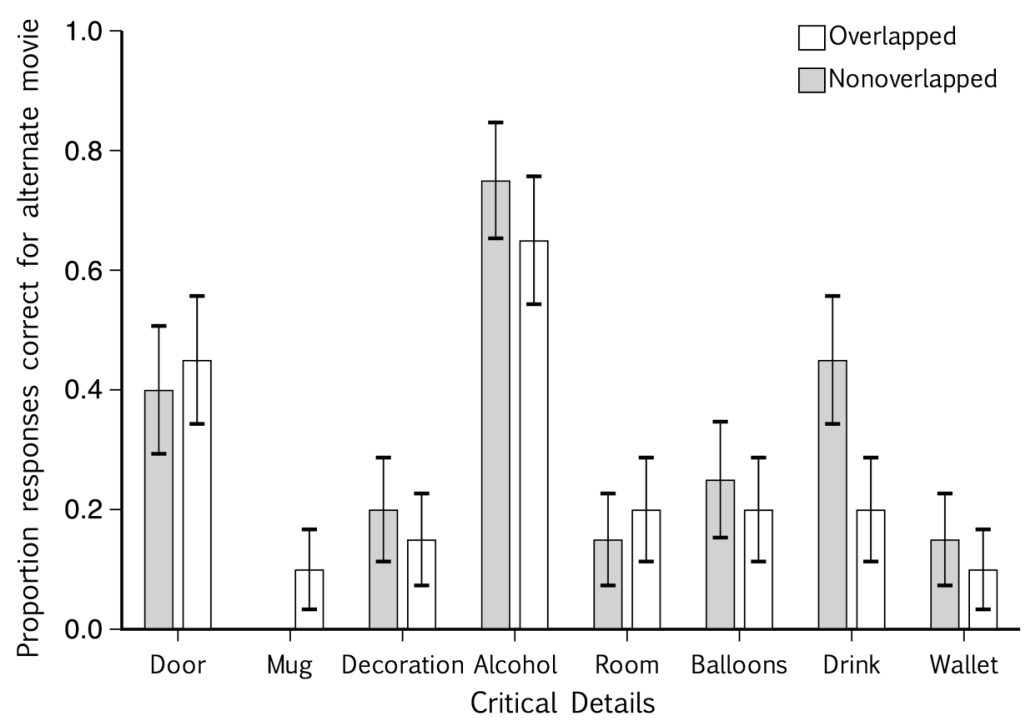

Figure 4.7. Proportion of second choices correct for alternate movie version for nonoverlapped and overlapped details. Error bars depict standard errors of the means. 


\section{Subjects' Explanations}

Finally, subjects wrote explanations for why they chose the second answers that they did. If subjects remembered both versions of a critical detail, they should say so when explaining their second response. However, there was not one case where a subject mentioned anything even vaguely related to seeing both versions of a detail. By far, the most common way that subjects selected their second choice was through logical reasoning; for $71 \%$ of the explanations given, subjects selected their second choice by reasoning that it made sense, or by excluding other alternatives. Other strategies that subjects used to make their second choices included guessing, beliefs about base rates, personal preferences and expectations.

\section{Summary}

Taken together, these two studies do not raise concerns about using the MORI technique to research discussion and memory; specifically, the results suggest that when people watch a movie via the MORI technique, they do not notice the alternate-blocked—movie version. In Experiment 3A, subjects were generally not suspicious that I might have shown them two different movies, and the vast majority of subjects did not notice any overlapping details. Additionally, the one subject who did notice overlapping images did not think the different images came from different movies. In Experiment ${ }_{3} \mathrm{~B}$, there was no evidence that subjects' memories of the critical details were affected by watching the movie via the MORI technique. Subjects were just as good at remembering overlapped and non-overlapped details, they were just as confident when remembering overlapped and non-overlapped details, they were no more likely to choose the correct answer from the alternate movie version for overlapped or nonoverlapped details, and their explanations did not raise any concern that they had noticed the alternate movie version. In short, there is no evidence to support 
reviewers' concerns that the MORI technique might inflate misinformation effects. Instead, the best explanation of the results from Experiments $2 \mathrm{~A}$ and $2 \mathrm{~B}$ is that people were influenced by misinformation that other people mentioned during discussion.

As discussed in Chapter 3, the SMF suggests that when people make source monitoring decisions they might make social judgments about the misinformation messenger-in these experiments, their discussion partners-to decide whether the information conveyed is reliable or not (Johnson et al., 1993; Lindsay, 2008; Smith \& Ellsworth, 1987). The findings from Experiments 2A and ${ }_{2} \mathrm{~B}$ - that people's memories are influenced by what other people tell themsuggest that people probably see others with whom they have shared experiences as a reliable source of information about those experiences. Yet, although people might generally see others with whom they have shared experiences as a valuable source of information, in some situations these perceptions might differ. Indeed, in Experiments $2 \mathrm{~A}$ and ${ }_{2} \mathrm{~B}$ people were not always influenced by what their partners told them. The SMF suggests one reason people might have resisted the influence of the misinformation is that they might have made unfavourable judgments about their discussion partners, and thus unfavourable judgments about the information conveyed.

There are a number of reasons that people might judge their discussion partner unfavourably; perhaps they might feel that their partner did not pay much attention, or did not seem to be very smart or very confident. People might also rely on stereotypes of age, appearance or gender-among other attributes_to make judgments about their partner's credibility (Hollingshead \& Fraidin, 2003; Wegner, 1987; Wegner et al., 1985; Wegner et al., 1991). Additionally, people might make social judgments based on how well they know the people that they talk to. In fact, as mentioned in Chapter 1, transactive memory research 
suggests that people might be more likely to trust what a friend or family member remembers than to trust what a complete stranger remembers. This trust might not only affect how much people believe what someone else says, but also how susceptible they will be to memory distortion based on what that person says. My next goal was to examine how people's relationships with their discussion partners might affect their source monitoring decisions and thus their susceptibility to false memories. 


\section{Chapter 5}

\section{Experiment 4: Romancing the Misinformation Messenger}

Generally, people talk about the past with other people that they know. Of course, in some situations—such as witnessing a crime or accident—people might talk with other people because they happened to be in the same place at the same time, rather than because they knew each other. In fact, Paterson, Chapman, and Kemp (2007) reported that although the majority of people who witnessed a crime or accident knew somebody else who witnessed the event, knowing another witness made no difference to whether people discussed the event or not. In other words, witnesses who knew another witness and witnesses who did not know another witness were just as likely to discuss the event. Yet, perhaps people who know each other might behave and respond differently in a conversation than people who do not know each other. Research shows that people's judgments about the misinformation messenger affect their susceptibility to misinformation (Dodd \& Bradshaw, 1980; Echterhoff, Hirst, et al., 2005; Vornik et al., 2003); one possibility is that people who know each other might make different social judgments about each other, thus affecting their source monitoring decisions and their susceptibility to false memories.

My main goal in this study was to see whether it made any difference to people's susceptibility to misinformation if the misinformation was conveyed by a known and trusted person, or if it was conveyed by a complete strangersomeone they had never met. To that end, I recruited romantic couples and pairs of strangers to take part in the study. I chose to use romantic couples because romantic relationships are based on components such as familiarity, trust and respect. By studying romantic couples, I could examine the effect of a collection of these kinds of factors that had arisen without experimental intervention. My primary interest was whether romantic partners would influence each other's 
memories more than strangers would. There are several reasons that intimate couples might influence each other more than strangers would.

First, as discussed in Chapter 1, people in intimate relationships develop transactive memory systems; members rely on each other's memories, and in doing so typically boost the amount of information they can remember, while minimising the effort each member has to exert to access the information (Hollingshead 1998a; 1998b; Wegner, 1987; Wegner et al., 1985; Wegner et al., 1991). Second, other research shows that an important predictor of success in romantic relationships is the willingness to be influenced by one's partner; more specifically, a husband who yields to his wife's influence is more likely to have a satisfying and lasting relationship (Gottman, Coan, Carrere, \& Swanson, 1998). Finally, people maintain relationships by negotiating a "shared reality" of their past experiences, and by agreeing on what "really" happened (Echterhoff, Higgins, et al., 2005; Hardin \& Higgins, 1996). Taken together, these three different lines of research lend support to the idea that members of couples may be more influenced by discussion with each other than strangers would be. Given that couples might rely on each other's memories for the day-to-day functioning of their relationship, and that they might be practiced in yielding to each other's influence and at incorporating each other's memories into their shared memories of the past, they might also be more likely than strangers to incorporate each other's misleading suggestions into their own subsequent memory reports.

My primary objective was to determine if people's memories would be more influenced by misinformation mentioned by their romantic partner than misinformation mentioned by a stranger. To address this question, I recruited romantic couples and pairs of strangers to take part in a study just like Experiment 2 A. Pairs of subjects watched the adapted Eric movie, discussed some 
aspects of the movie together and then completed a surprise memory test independently. If couples were worse than strangers at remembering discussed details on the final recognition test it would provide support for the idea that people's memories are more influenced by misleading suggestions made by someone they know and trust than someone they do not know.

\section{Method}

\section{Subjects}

In total, 64 people took part in the study; 32 took part with someone who they had never met and $3^{2}$ took part with their romantic partner. ${ }^{7}$ I recruited subjects by advertising around the university campus and in staff and student publications. In advertisements targeting romantic partners, I specifically asked for "monogamous heterosexual couples" who had been together for at least three months. I chose three months as the minimum relationship duration because it was consistent with other studies (for example, Wegner et al., 1991); however, I did not obtain any further information on relationship duration. All pairs were made up of one male and one female. Subjects received a voucher to thank them for their time.

\section{Design}

The experiment used a 2 x 2 mixed design: subjects either took part with their romantic partner or with a stranger, and as in Experiments $2 \mathrm{~A}$ and $2 \mathrm{~B}$, half of the critical details acted as discussed details, and half acted as nondiscussed details.

\section{Materials and Procedure}

The experiment was identical to Experiment $2 \mathrm{~A}$, except for one change. In order to decrease the magnitude of the misinformation effect—so that I could

\footnotetext{
${ }^{7}$ A further two couples and one stranger pair were not included in the study because one member in each pair did not follow instructions; one person repeatedly tilted his head during the movie, and the other two made no attempt at filler tasks. Another two stranger pairs were excluded because of a fault with the digital voice recorder. The data I present is for the remaining 64 subjects.
} 
examine differences between the two relationship conditions-I shortened the duration of the filler tasks to 12 min and 3 min respectively.

Results and Discussion

In total, 16 romantic couples and 16 stranger-pairs completed the study; as in the previous experiments, all data were analysed on an individual level, yielding a total of 64 subjects. Consistent with Experiments $1 \mathrm{~A}, 1 \mathrm{~B},{ }_{2} \mathrm{~A}$ and ${ }_{2} \mathrm{~B}$, subjects performed well on the 12 filler details, and there were no differences based on whether subjects took part with their romantic partner or a stranger, $M=10.13$ correct (84\%), $S D=1.24 ; M=10.22$ correct (85\%), $S D=1.16$ respectively; $t<1, n S$.

\section{Discussion Duration}

Also consistent with Experiments $2 \mathrm{~A}$ and ${ }_{2} \mathrm{~B}$, no one took the maximum time allowed to discuss the questions; discussion duration ranged 2:54 to 8:03 $\mathrm{min}, M=$ 5:06 min, $S D=1: 05$. Couples did take slightly longer than stranger pairs to complete the discussion, however this difference was not significant, $M=5: 24$ $\min , S D=0: 51 ; M=4: 49 \min , S D=1: 14 ; t(30)=1.57, p=.13$. In fact, there was a marginal trend that couples took longer than strangers to discuss both filler questions- $M=26.54 \mathrm{~s}, S D=4.92 ; M=23.67 \mathrm{~s}, S D=7.77$ - and critical questions$M=27.84 \mathrm{~s}, S D=5.92 ; M=24.8 \mathrm{o} \mathrm{s}, S D=7.25, F(1,30)=2.69, p=.11$, perhaps suggesting that couples were more comfortable discussing the details together. Despite couples taking longer on average, couples and strangers received exactly the same number of warnings for their discussions about critical and filler questions; in total subjects were told that they had 10 seconds remaining 14 times when discussing filler questions (out of a possible $256 ; 5 \%$ ), and 4 times when discussing critical questions (out of a possible $128 ; 3 \%$ ). Unlike the previous experiments, both couples and strangers took only as long to discuss critical questions as filler questions, $F<1$, ns. Collapsing across couples and strangers, discussion time for critical details ranged from 6 s to $75 \mathrm{~s}, M=26.32 \mathrm{~s}, S D=6.8 \mathrm{o}$, 
and discussion time for filler details ranged from $5 \mathrm{~s}$ to $79 \mathrm{~s}, M=25.11 \mathrm{~s}, S D=6.66$. Exposure to Misinformation

Subjects were exposed to between o and 4 misleading details, $M=2.41, S D=$ o.9o; in total they were exposed to misinformation 154 out of a possible 256 times $(60 \%)$. Couples and strangers were equally likely to be exposed to misinformation; of these 154 instances members of couples were exposed to misinformation 76 times $(49 \%)$ and strangers were exposed to misinformation the remaining 78 times $(51 \%)$.

Remembering Discussed Details

I next calculated the proportion of correct responses each subject gave for discussed and nondiscussed details in the same way as the previous experiments; these data are displayed in Figure 5.1.

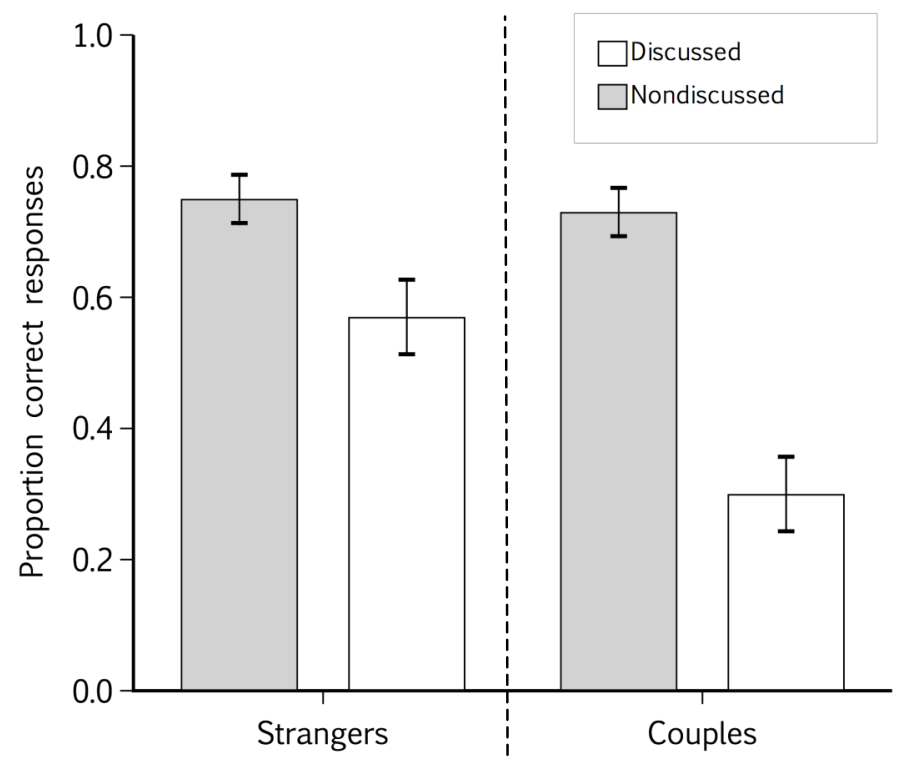

Figure 5.1. Proportion of correct responses for nondiscussed and discussed details by relationship. Error bars depict standard errors of the means

Did couples wield more influence over each other's memories than strangers did? As Figure 5.1 shows, the answer is yes. Both couples and strangers were good at remembering nondiscussed details and not as good at remembering discussed 
details. Furthermore, couples were worse than strangers at remembering discussed details. In other words, on the independent test, subjects reported seeing details that they only heard about during the discussion, and they were even more likely to do so when they heard about those details from their romantic partner rather than from a stranger, $F(1,61)=5.39, p=.02, f=0.27$.

Consistent with Experiments $2 \mathrm{~A}$ and ${ }_{2} \mathrm{~B}$, as illustrated in Figure 5.2, subjects were more likely to give the correct response for critical details when they acted as nondiscussed details, $t(7)=5.20, p<.01$, Cohen's $d=1.84$.

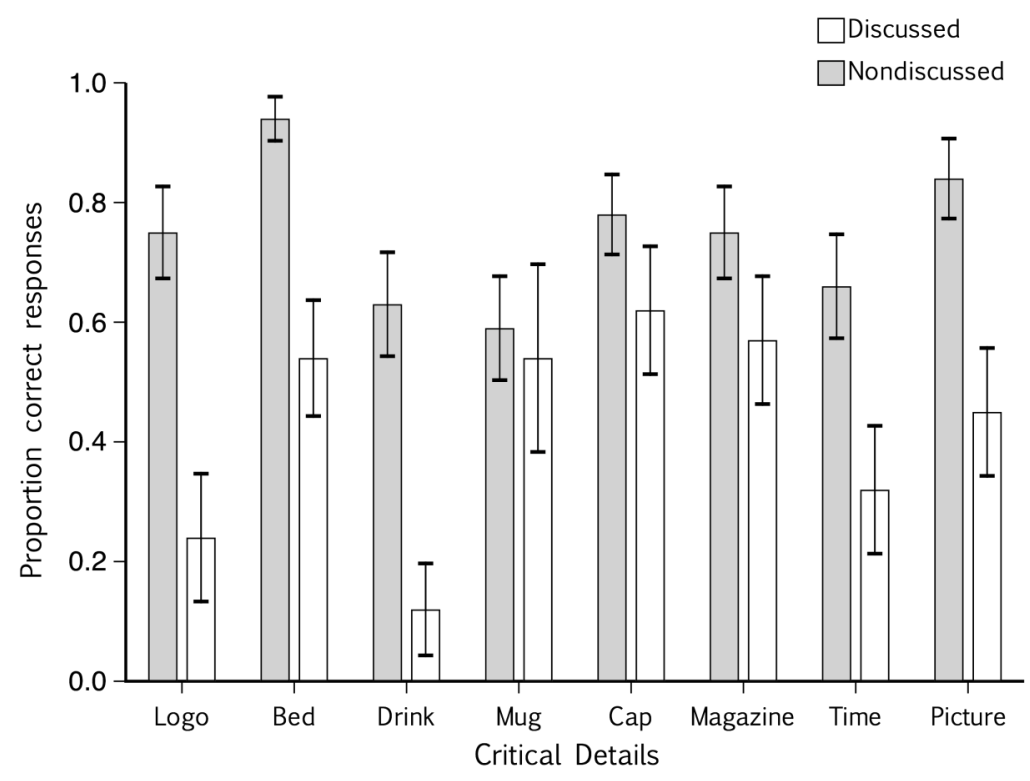

Figure 5.2. Proportion of correct nondiscussed and discussed responses for each critical detail. Error bars depict standard errors of the means.

Following Experiments $2 \mathrm{~A}$ and $2 \mathrm{~B}$, I next focused on subjects who were exposed to at least two misleading details. Of these 53 subjects, $15(28 \%)$ were misled by everything their partner told them, 8 (15\%) were not misled by anything their partner told them, and the remaining 30 (57\%) were misled about some details but not others. Interestingly, these patterns were quite different for subjects who took part with their romantic partner and subjects who took part with a stranger; the proportion of couples and strangers who were never misled, 
sometimes misled, and always misled are displayed in Table 5.1. In short, more couples were always misled by their partner's suggestions, and more strangers were never misled.

Table 5.1

Proportion of strangers and couples who-when exposed to at least two misleading details-were never, sometimes, and always misled

\begin{tabular}{lcc} 
& \multicolumn{3}{c}{ RELATIONSHIP } \\
& $\begin{array}{c}\text { Couples } \\
(n=25)\end{array}$ & $\begin{array}{c}\text { Strangers } \\
(n=28)\end{array}$ \\
\hline Never Misled & $.08 \quad(2)$ & $.22 \quad(6)$ \\
Sometimes Misled & $.48(12)$ & $.64(18)$ \\
Always Misled & $.44(11)$ & $.14 \quad(4)$
\end{tabular}

Taken together, these results suggest that the relationships people have with each other might determine how susceptible they are to misinformation conveyed in a discussion. However, there is another possible explanation for the results-recall the trend for couples to take longer than stranger pairs to complete the discussion, potentially increasing the retention interval for couples over strangers. A well-established finding in the misinformation effect literature is that the longer the retention interval between the event and the memory test, the more misled subjects tend to be (Loftus et al., 1978). Perhaps, then, couples were more misled not because of their relationship with each other, but as a consequence of the increased retention interval, which caused poorer memory of the event and thus increased their susceptibility to the misleading suggestions. But, if couples did have inferior memories of the event, they should be worse than strangers at remembering nondiscussed details. In fact, they were not: 
couples and strangers remembered nondiscussed details equally well, $M=.73, S D$ $=.25 ; M=.75, S D=.22 ; t<1, n s$, suggesting that the increased retention interval did not cause the effect.

Another counterexplanation for the results is that the couples used the extra discussion time to present more evidence and to persuade their partner of their own views, and this extra effort meant they were more successful at influencing their partners. In other words, the longer couples discussed the critical details, the more persuaded and the less accurate they should be. To examine this possibility, I conducted a correlation analysis between the time couples took to discuss critical details where they were exposed to misinformation and their accuracy at remembering those details on the recognition test. Although there was a marginal trend, it was in the opposite direction: the longer couples spent discussing the critical details, the more accurate they were on the final test, $r=$ $.32, p=.08$. In short, there was no support for the hypothesis that increased discussion duration led members of couples to be more persuaded by their partners. Instead, the results suggest that the longer couples took to discuss the critical questions, the more likely they were to resist the influence of their partner's misleading suggestions.

\section{Confidence}

I next calculated subjects' confidence for their responses to discussed and nondiscussed details; these results are displayed in Figure 5.3. The results are consistent with the previous experiments, and show that subjects were moderately confident in all their responses. Figure 5.3 also shows that there was no interaction between relationship and discussion: whether subjects were paired with their romantic partner or a stranger had no effect on their confidence when remembering discussed or nondiscussed details, $F<1$, ns. Yet there was trend toward a main effect for relationship: couples were more confident about all 
their answers than strangers were, $F(1,60)=3.30, p=.07, f=0.23$. There was no effect of discussion: subjects were just as confident when remembering discussed and nondiscussed details, $F(1,60)=2.26, p=.14$.

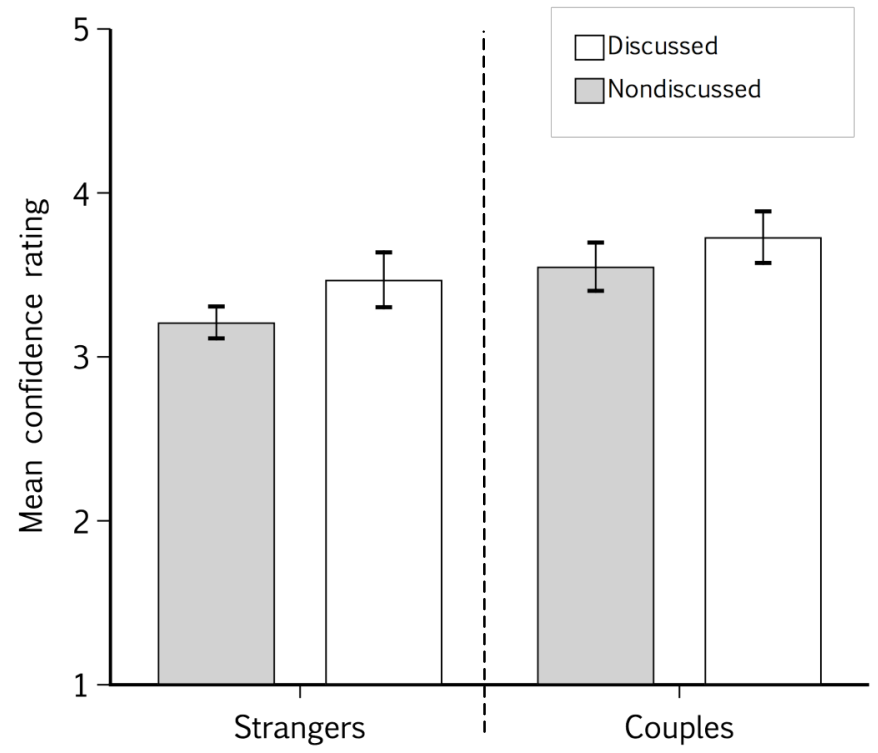

Figure 5.3. Mean confidence for nondiscussed and discussed details by relationship. Error bars depict standard errors of the means.

Next, I examined subjects' confidence in their true memories (when they gave the correct response on nondiscussed details) and their false memories (when they gave the incorrect response on discussed details); these data appear in Figure 5.4. As the figure illustrates, there was no interaction between relationship and accuracy: whether subjects were paired with their romantic partner or a stranger had no effect on their confidence for either true memories or false memories, $F(1,49)=1.30, p=.26$. However, there was a main effect for relationship type: members of couples were more confident about their memories than strangers were, $F(1,49)=12.83, p<.01, f=0.45$. This result fits well with the trend for overall confidence; couples were more confident in their responses whether or not they discussed those specific details and whether or not their responses were correct. Perhaps couples were used to verifying the past 
with each other and-through previous experience-the verification boosted their confidence in memory for all details of the event, regardless of whether they actually discussed those specific details. Finally, consistent with Experiments $2 \mathrm{~A}$ and $2 \mathrm{~B}$, Figure 5.4 also shows that there was a main effect for accuracy: couples and strangers were more confident about their true than their false memories, $F(1,49)=8.76, p<.01, f=0.42$.

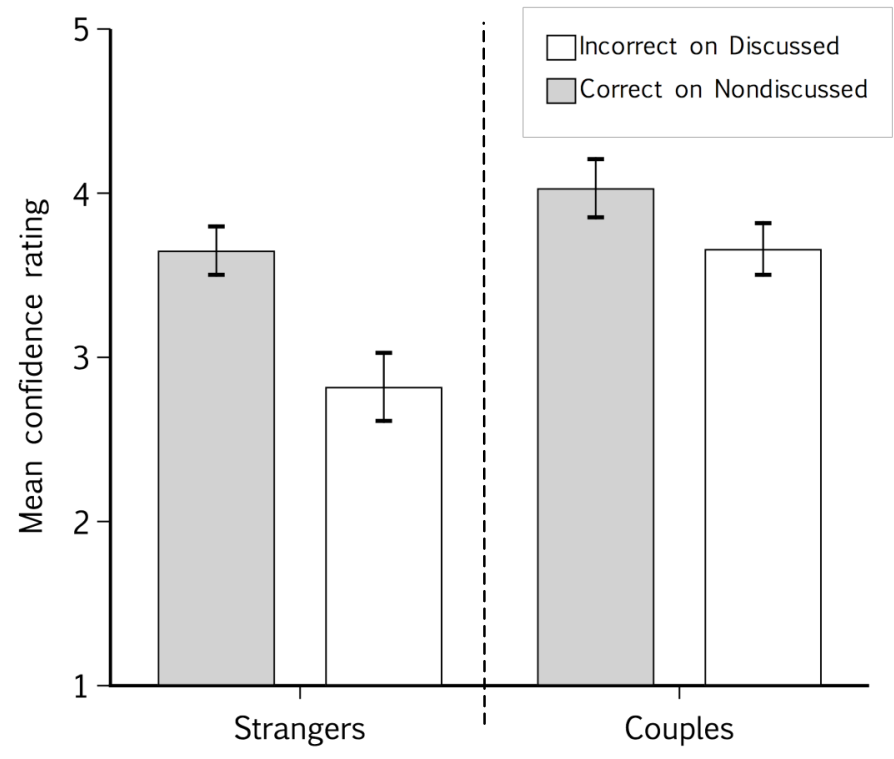

Figure 5.4. Mean confidence for correct responses on nondiscussed details (true memories) and incorrect responses on discussed details (false memories) by relationship. Error bars depict standard errors of the means.

\section{Features of the Discussion}

As in the previous experiments, I used transcripts of subjects' voicefiles to determine what happened during their discussions; for each of the 154 instances that subjects were exposed to the misinformation I examined four discussion features: if the subject spoke first; if the subject disputed what his or her partner said; if what the subject said was disputed by his or her partner; and if the subject used the misleading detail as an answer to the critical discussion question. Like in the previous experiments, of the 154 instances that subjects were exposed to 
misinformation, there were only 19 instances $(12 \%)$ where one subject disputed the other, and the other did not reciprocate. In other words these two factorswhether the subject disputed his or her partner, and whether the subject was disputed by his or her partner-were not different enough to consider independently; instead, following Experiment $2 \mathrm{~B}$, I examined only whether subjects disputed what their partners said. I calculated the proportion of instances that each discussion feature occurred and the proportion of times that subjects went on to be misled; these data appear in Table 5.2.

Table $5 \cdot 2$

Proportion discussion feature occurrence and subsequent proportion of subjects misled for 154 instances that subjects were exposed to misinformation

\begin{tabular}{|c|c|c|c|c|}
\hline \multirow[b]{2}{*}{ Discussion Features } & \multicolumn{2}{|c|}{$\begin{array}{c}\text { STRANGERS } \\
(n=78 \text { cases })\end{array}$} & \multicolumn{2}{|c|}{$\begin{array}{c}\text { COUPLES } \\
(n=76 \text { cases })\end{array}$} \\
\hline & $\begin{array}{c}\text { Feature } \\
\text { (n cases) }\end{array}$ & $\begin{array}{c}\text { Misled } \\
\text { (n cases) }\end{array}$ & $\begin{array}{l}\text { Feature } \\
\text { (n cases) }\end{array}$ & $\begin{array}{c}\text { Misled } \\
\text { (n cases) }\end{array}$ \\
\hline
\end{tabular}

Answer

$\begin{array}{lllll}\text { Misinformation } \quad .56(44) & .75(33) \quad .55(42) \quad 1.00(42)\end{array}$

$\begin{array}{lllll}\text { Alternative } & .44(34) & .06(2) & .45(34) & .29(10)\end{array}$

\section{Dispute}

$\begin{array}{lllll}\text { Dispute } & .46(36) & .19(7) & .51(39) & .44(17)\end{array}$

$\begin{array}{lllll}\text { No Dispute } & .54(42) & .67(28) & .49(37) & .95 \text { (35) }\end{array}$

Speaking Order

$\begin{array}{lllll}\text { Spoke First } & .33(26) & .27(7) & .30(23) & .43(10)\end{array}$

$\begin{array}{lllll}\text { Spoke Second } & .67(52) & .54(28) & .70(53) & .79(42)\end{array}$

OVERALL PERFORMANCE $.45(35)$ $.68(52)$

Note. Proportion Correct $=1-$ Proportion Misled. For strangers ( $\mathrm{n}$ cases Correct $=78-\mathrm{n}$ cases Misled); for couples ( $\mathrm{n}$ cases Correct $=76-\mathrm{n}$ cases Misled). 
As Table 5.2 illustrates, each of the discussion features occurred at similar rates in couples' and strangers' discussions, but their responses were quite different. The first two lines of Table 5.2 show that-consistent with Experiments $2 \mathrm{~A}$ and $2 \mathrm{~B}$ - people were misled more often if they used the misinformation as their discussion answer; this pattern was especially true of couples. In fact, not one subject who took part with their romantic partner appeared to exhibit normative influence: instead, every time that members of couples used the misinformation as an answer during the discussion, they used the same information as an answer on the independent test. However, strangers did appear to exhibit normative influence some of the time, using the misinformation during discussion, but later reporting something different (Deutsch \& Gerard, 1955). Consistent with the previous experiments, subjects sometimes used the misinformation as their answer during the independent test, even though they gave a different answer during the discussion, and again this behaviour was especially true for members of couples.

Recall that couples took longer than strangers to complete their discussions. One possible explanation for this difference is that the couples were more comfortable arguing with one another. However, as the third line of Table 5.2 shows, couples and strangers were just as likely as to dispute their partner's suggestions. In other words couples' longer discussion durations cannot be explained by a greater willingness to argue.

Although couples and strangers disputed each other equally often, the outcomes were quite different. As Table 5.2 illustrates, the vast majority of the time that couples did not dispute their partner's suggestions, they were subsequently misled. Additionally, couples who disputed their partners were misled twice as often as strangers who disputed their partners. Finally, both 
couples and strangers were misled less often when they spoke first in the discussion of a critical detail. However, consistent with the other results, couples were still misled more often than strangers when they spoke first.

In sum, even though members of couples and of stranger pairs were equally exposed to misinformation, and even though the features of their discussions were very similar, these data suggest that subjects' experiences of the discussion were influenced by their relationships with each other. Couples never went along with their partner to be polite, or to "keep the peace"-or if they did, they maintained that behaviour even in an independent memory test. Furthermore, when couples refused to answer with the misinformation during discussion, they were more likely than strangers to use that same information at a later stage, on the independent test. These results suggest that couples might be more inclined than strangers to take their partner's claims as evidence of reality.

\section{Predicting False Memories}

As in the previous experiments, I next examined if any features of the discussion predicted whether subjects were misled on a particular detail. I conducted the same two analyses, the all-items regression, which-consistent with Gabbert et al.'s (2006) approach-included all instances that subjects talked about critical details, regardless of whether they were exposed to misinformation; and the exposure-only regression, which addressed Lindsay's (2007) concerns by only including instances where subjects were actually exposed to misinformation. For this experiment, I included an additional predictor in the analyses: whether subjects took part with their romantic partner or a stranger.

All-items regression. When all 256 critical details that were asked about in the discussion were included in the regression-regardless of whether subjects were exposed to the misinformation-there were three predictors of whether a subject 
was misled. First, the odds ratio showed that subjects who took part with their romantic partner were nearly six times more likely to be misled than subjects who took part with a stranger, $\chi^{2}(1, N=256)=12.02, p<.01$. Second, consistent with the previous experiments, when subjects used the misinformation as an answer during the discussion they were nearly 70 times more likely to be misled than if they answered with an alternative, $\chi^{2}(1, N=256)=65.03, p<.01$. Finally, consistent with Gabbert et al. (2006), when subjects spoke first they were nearly four times less likely to be misled, $\chi^{2}(1, N=256)=9.85, p<.01$.

Exposure-only regression. When examining only the 154 instances in which subjects were exposed to misinformation, two factors predicted whether they would be misled. First, subjects who took part with their romantic partner were 14 times more likely to be misled than those who took part with a stranger, $\chi^{2}(1$, $N=154)=11.94, p<.01$. Second, when subjects used the misinformation as their discussion answer they were 87 times more likely to be misled than when they used an alternative, $\chi^{2}(1, N=154)=34.07, p<.01$. Additionally, there was a marginally significant predictor-subjects who spoke first when discussing a critical detail were less likely to be misled, $\chi^{2}(1, N=154)=2.84, p=.09$, suggesting that speaking order might in fact play some role in predicting susceptibility to false memories.

In sum, consistent with the previous experiments, subjects were most likely to be misled when they answered with the misinformation during discussion. Additionally, subjects were more likely to be misled when the misleading information came from their romantic partner rather than a stranger. Interestingly, the marginal trend suggests that speaking order might actually play some role in susceptibility to false memories, not just in exposure to misinformation. However, speaking order was still a much better predictor of 
being misled when exposure to misinformation was not considered-in the allitems regression-suggesting that speaking order is most important because it is related to exposure to misinformation.

Predicting exposure regression. Finally, as in the previous experiments, I conducted another regression to examine whether any features of the discussion predicted whether subjects were exposed to misinformation in the first place; the results were very similar to Experiment $2 \mathrm{~B}$. The odds ratios showed that subjects who disputed what their partner said were 4 times more likely to be exposed to misinformation, $\chi^{2}(1, N=256)=18.37, p<.01$, and subjects who spoke first were four times less likely to be exposed to misinformation, $\chi^{2}(1, N=256)=20.07, p<$ .o1. Additionally, there was an interaction between the two predictors: subjects who spoke first and disputed what their partners said were three times less likely to be exposed to the misinformation, $\chi^{2}(1, N=256)=13.06, p<.01$. Subjects who took part with their romantic partner were just as likely as those who took part with a stranger to be exposed to misinformation.

\section{Summary}

In sum, the results of this study show that relationships matter; susceptibility to false memories is affected not only by people's judgments about the misinformation messenger, but also by their relationship with the misinformation messenger. These results show that when people discuss the past with an intimate partner they might become not only less accurate but also more confident about their memories.

The results fit with the social psychology literature suggesting that couples might be practiced in accepting their partner's influence and in relying on each other's memories (Gottman et al., 1998; Hardin \& Higgins, 1996; Hollingshead 1998a; 1998b; Wegner et al., 1991). Although the findings fit with these studies, I did not obtain any measure of relationship duration, relationship satisfaction, 
areas of expertise within the relationship (evidence of specific transactive memory systems), nor gauge how the couples talked about their shared past. It might be that measures such as these can predict whether people in romantic relationships will be more or less susceptible to false memories.

When couples rely on each other's memories they experience an advantage by increasing the amount of information they can access while reducing the amount of effort required. However, this research shows that couples might also experience a disadvantage in memory accuracy when their partner conveys incorrect information. Often, when people discuss events together, they do so with someone they know rather than with a stranger. If people are even more susceptible to false memories when someone they know conveys the misleading information, then memory conformity studies to date (which do not account for relationship) may underestimate the true effect of discussion on memory (but see Hope, Ost, Gabbert, Healey, \& Lenton, 2008).

The results also fit with work showing the importance of the source of misinformation in determining the consequences for memory; people rely on their knowledge and judgments about each other to decide if the information conveyed is good or bad. For instance, misleading suggestions made by a reliable, credible, trustworthy, attractive or powerful messenger might be more influential than suggestions made by an unreliable, non-credible, untrustworthy, unattractive, or weak messenger (Dodd \& Bradshaw, 1980; Echterhoff, Hirst, et al., 2005; Smith \& Ellsworth, 1987; Vornik et al., 2003). The major result of this study - that people were more influenced by their romantic partner's misleading suggestions than by a stranger's misleading suggestions-suggests that people probably make more favourable social judgments about their romantic partners than about strangers. Yet, given that a sizeable proportion of subjects were influenced by some misleading suggestions while resisting others, it seems that 
rather than simply making broad judgments about whether the messenger is good or bad, people might make specific judgments depending on the content and context of each suggestion. Indeed, transactive memory research suggests that when people know each other very well they might judge information from specific domains to be more or less valuable depending on the messenger's expertise. For instance, returning to the example from Chapter 1, my partner would not trust my recall of driving directions, but he would trust my recall of a phone number.

In fact, some of the statements subjects made during the discussion suggest that they considered who was more expert in the specific area to decide whose memory to trust. For instance, when one couple discussed the colour of the couch, the male subject said, "You're the colour expert", and used the expert's suggestion as his discussion answer. Similarly, when another couple discussed whether the bed was made, the female repeatedly stated that it was not; the male replied, "I thought it was made but I tend not to notice things like mess and unmade beds..." In fact, these kinds of statements were not limited to couples. For instance, one stranger communicated his lack of expertise. Specifically, when discussing the colour of the walls, he said, "I'm not that into interior design" and reiterated the same point when discussing the curtains, “...interior design—not my area!' In short, consistent with transactive memory theory, subjects seemed to consider each other's expertise as a relevant factor during the discussion. Romantic couples might have been more familiar with each other's expertise, whereas stranger pairs would have had to inform each other what they were good at or rely on stereotypes to make these decisions (Hollingshead \& Fraidin, 2003; Wegner, 1987; Wegner et al., 1985; Wegner et al., 1991). Regardless of whether they relied on knowledge or assumptions about expertise, subjects seemed to use these expertise judgments to infer whose memory would be more 
credible, and thus whose memory to trust.

In everyday life, people probably make these kinds of credibility judgments by considering a whole host of factors beyond expertise. For instance, if a group of strangers witnessed an accident, each person might make different judgments about the reliability of different witnesses based on different criteria. Perhaps the man who forgot to put his glasses on might see himself as less reliable than the man who was up close as the accident happened; similarly, the woman who phoned for help might see herself as more knowledgeable than the woman who was chatting on her phone when the accident happened. Perhaps a teenage witness might see herself as more credible than a young child or an elderly person that was at the scene. In short, people's expectations about their own credibility relative to the misinformation messenger's credibility might be based on a wide variety of factors. Given that people might use credibility judgments to infer how reliable a given piece of information is, perhaps these judgments and expectations might also affect people's susceptibility to misleading suggestions. For instance, perhaps the man who forgot his glasses might be more susceptible to misinformation conveyed by other witnesses, and the woman who called for help might be less susceptible. These ideas motivated my next study where my goal was to examine whether people's expectations about their own abilities and about their discussion partner's abilities would affect their source monitoring decisions, and thus their susceptibility to false memories. 


\section{Chapter 6}

\section{Experiment 5: Great and Not-So-Great Expectations}

People's perceptions of the misinformation messenger affect their susceptibility to false memories (Dodd \& Bradshaw, 1980; Ceci et al., 1987;

Echterhoff, Hirst, et al., 2005; Vornik et al., 2003). Yet it seems unlikely that these perceptions work in isolation. For instance, people who judge themselves very favourably might be less open to the influence of misleading suggestions than people who judge themselves less favourably. Put another way, people's perceptions of themselves might also play a role in determining their susceptibility to misleading suggestions.

In fact, some misinformation effect research shows that people's expectations about themselves can affect their susceptibility to false memories. Assefi and Garry (2003) conducted a misinformation effect study and provided subjects with placebo alcohol. Half of the people in the study were told they drank tonic water, and the other half were told that they drank alcohol. Everyone actually drank the same thing: tonic water. Yet, people who were told they drank alcohol were more influenced by the misleading suggestions. One explanation for this result is that people who thought they drank alcohol subsequently expected their memory to be poor and thus were more likely to capitulate to the misinformation messenger-in this case, the presumably sober experimenter who provided the written description. In short, people's expectations about their own abilities might lead them to be more susceptible to the influence of misleading suggestions. Several subsequent studies have shown that these expectancy effects also operate in the opposite direction, leading people to be less susceptible to the influence of misleading suggestions. (Clifasefi, Garry, Harper, Sharman, \& Sutherland, 2007; Parker, Garry, Engle, Harper, \& Clifasefi, 2008). Specifically, instead of telling subjects that they drank alcohol, Clifasefi et al. told half of the 
subjects in their study that they took a cognitive enhancing drug; the other subjects were told that they took an inactive placebo. In fact there was no drug: everyone took a placebo made of baking soda and water. Yet, people who were told they had taken the active drug were less influenced by the misleading suggestions. Perhaps people who thought they took the drug subsequently expected their memories to be very good, and thus they were less likely to capitulate to the misinformation messenger's suggestions.

In sum—when examined independently—people's judgments about the misinformation messenger and people's expectations about themselves can lead them to be more or less influenced by misinformation conveyed in a narrative description. In the next two experiments, I examined these factors in combination to see whether people's expectations about themselves and their expectations about other people work together to determine their susceptibility to misinformation conveyed in a social interaction.

To date, two memory conformity studies have manipulated subjects' perceptions of their own and their discussion partner's credibility; in both studies the supposedly less credible subjects were more susceptible to false memories than the supposedly more credible subjects (Gabbert et al., 2007; Skagerberg \& Wright, 2008). However, in both studies, within subject-pairs the balance was always tipped in one person's favour: one member was perceived to have an advantage and the other was perceived to have a disadvantage. More specifically, one subject was told his or her partner had seen a target picture for more time and the other was told his or her partner had seen it for less time (although actual viewing time was equivalent; Gabbert et al., 2007); similarly, one person was allocated a low power role and the other was allocated a high power role (Skagerberg \& Wright, 2008).

Although these studies showed that people in the apparently disadvantaged 
roles were more susceptible to false memories, neither study had a baseline measure of susceptibility to false memories. In fact, the effects might actually occur because people in the supposedly advantaged roles were less susceptible to false memories. Additionally, the studies do not tell us whether perceptions of the self or perceptions of the misinformation messenger drive the effect. For example, we do not know what would happen if the misinformation messenger and the person were both highly credible, or if they both lacked credibility.

I wondered whether the effect found by Gabbert et al. (2007) and Skagerberg and Wright (2008) was driven by some people being more susceptible to false memories, some people being less susceptible to false memories, or a combination of the two. Additionally, I wondered what mechanisms might drive the effect. If people in the disadvantaged roles were more susceptible to misinformation, it might be because they made favourable judgments of the misinformation messenger, because they made unfavourable judgments of themselves, or because of some interaction between the two. Likewise, if people in the advantaged roles were less susceptible to misinformation, it might be because they made unfavourable judgments of the misinformation messenger, because they made favourable judgments of themselves, or because of some interaction between the two.

To answer these questions, I conducted a study just like Experiment ${ }_{2} \mathrm{~B}$, but tried leading subject-pairs to believe that each person would have either good or slightly degraded vision during the movie. Thus, within pairs, subjects would expect to be more, equally, or less able than their partner to see the movie. If subjects' expectations about their abilities and their partner's abilities affected their susceptibility to misleading suggestions, we should see differences in their memory for discussed details. These differences might occur in one of three ways. First, if susceptibility to false memories is driven by people's expectations 
about the misinformation messenger, then people who are told their discussion partner-the misinformation messenger-has good vision should be more misled than people who are told their discussion partner has degraded vision, regardless of what they are told about their own vision. Second, if susceptibility to false memories is driven by people's expectations about themselves, then people who are told they have degraded vision should be more misled than people who are told they have good vision, regardless of what they are told about their partner's vision. Finally, if susceptibility to false memories is affected by people's expectations about themselves in combination with their expectations about the misinformation messenger, then we should see an interaction between the two factors. Additionally, any differences between the groups might be driven by one group being more susceptible to false memories, one group being less susceptible to false memories, or a combination of the two.

\section{Method}

\section{Subjects}

In total, 160 IPRP students took part in pairs in return for course credit.

\section{Design}

The experiment used a $2 \times 2 \times 2$ mixed design: subjects were told that their ability to see the movie was either good or slightly degraded, and that their partner's ability was either the same as or different from their own. As in the previous experiments, for every subject, half of the critical details from the movie acted as discussed details and the other half acted as nondiscussed details.

\section{Materials and Procedure}

The experiment was identical to Experiment $2 \mathrm{~B}$, except for two changes. As before, subjects had the impression that they might be assigned to wear $35 \%$, $55 \%, 75 \%$ or $95 \%$ acuity glasses. But unlike the earlier experiments, instead of telling subjects they would be in the $95 \%$ acuity condition, each subject was 
assigned to wear either $75 \%$ acuity glasses (slightly degraded vision) or $95 \%$ acuity glasses (good vision). Regardless of their acuity label, none of the glasses degraded subjects' vision in any way: each pair of glasses was made of either horizontal polarising filter, or vertical polarising filter, and allowed each subject to see one movie while blocking the other from view. Prior to the movie, subjects chose an envelope to see which condition they would be in, and were handed glasses from the corresponding box. The envelopes were arranged to ensure that subjects were assigned to the correct condition. There were four possible expectancy combinations based on the glasses that subjects were given: [1] both the subject and his or her partner expected to have degraded vision; [2] both the subject and his or her partner expected to have good vision; [3] the subject expected his or her partner to have superior vision; or [4] the subject expected his or her partner to have inferior vision (see Table 6.1).

Table 6.1.

Expectancy combinations based on subject-pairs' assigned glasses

\begin{tabular}{|c|c|c|c|}
\hline & & \multicolumn{2}{|c|}{ PARTNER'S GLASSES } \\
\hline & & 75\% Acuity & 95\% Acuity \\
\hline \multirow{2}{*}{$\begin{array}{c}\text { OWN } \\
\text { GLASSES }\end{array}$} & 75\% Acuity & $\begin{array}{c}\text { Both Degraded } \\
\text { Vision }\end{array}$ & $\begin{array}{c}\text { Messenger } \\
\text { Superior Vision }\end{array}$ \\
\hline & 95\% Acuity & $\begin{array}{c}\text { Messenger } \\
\text { Inferior Vision }\end{array}$ & $\begin{array}{l}\text { Both Good } \\
\text { Vision }\end{array}$ \\
\hline
\end{tabular}

The second change I made from Experiment ${ }_{2} \mathrm{~B}$ was to add a manipulation check following the recognition test to see whether subjects believed that the glasses degraded (or did not degrade) their vision. Subjects rated their vision of 
the movie on five different dimensions: overall vision, sharpness, brightness, contrast and vibrancy; they rated each aspect a 5-point Likert scale where 1 was low and 5 was high. A sixth scale asked subjects whether they experienced any sensory compensation-if they noticed their other senses being more switched on than usual while their vision was degraded; subjects rated their sensory compensation on another 5-point Likert scale, where $1=$ "no different to normal' and 5 = "extremely switched on". Finally, subjects were asked to report which type of glasses they wore and which type of glasses their partner wore.

Results and Discussion

In total 8o subject-pairs completed the study, but as in the previous experiments, all data were analysed on an individual level, yielding a total of 160 subjects. Consistent with the previous experiments, subjects were good at remembering the twelve filler details, and there were no differences based on whether subjects wore $75 \%$ or $95 \%$ glasses, $M=8.89$ correct $(74 \%), S D=1.48 ; M=$ 8.74 correct $(73 \%), S D=1.51, t<1, n s$.

\section{Manipulation Checks}

To examine whether subjects believed that the glasses degraded (or did not degrade) their vision, for each subject I calculated an overall sensory impressions score, based on the mean of their five vision ratings. Subjects who wore $75 \%$ glasses rated their impressions of the movie as worse than subjects who wore 95\% glasses, suggesting that the manipulation did influence subjects' beliefs about their ability to see, $M=2.95, S D=0.62 ; M=3.25, S D=0.65 ; t(156)=3.01, p<$ .01, Cohen's $d=0.48$.

I next examined subjects' responses to the sensory compensation measure. If the manipulation was effective, subjects who were told that their vision was degraded should give higher ratings on this item. In fact, there was no difference between subjects who wore $75 \%$ glasses and subjects who wore $95 \%$ glasses, 
suggesting that either this item was a poor measure of the manipulation or the manipulation was not effective, $M=2.26, S D=1.20 ; M=2.04 ; S D=1.16 ; t(156)=$ $1.20, p=.23$.

\section{Discussion Duration}

As in the previous experiments, there was no evidence that subjects were rushed to choose answers during the discussion. Pairs took between 2:18 min and 7:17 min to complete the discussion, well under the 12 min allowed, $M=4: 17 \mathrm{~min}$, $S D=1: 06$ min. It made no difference to discussion duration whether subject-pairs wore the same or different glasses, $M=4: 24 \mathrm{~min}, S D=1: 07 ; M=4: 10 \mathrm{~min}, S D=$ 1:05; $t<1$, ns. Additionally, subjects were given very few warnings to remind them they had 10 sec left. Pairs who wore the same glasses received 18 warnings when discussing filler details (out of a possible 320 instances; 6\%) and 3 warnings when discussing critical details (out of a possible 16o instances; $2 \%$ ). Pairs who wore different glasses received 12 warnings when discussing filler details (out of a possible 320 instances; $4 \%$ ) and only 1 warning while discussing a critical detail (out of a possible 160 instances; < 1\%). Collapsing across conditions, subjects took longer to discuss critical details than filler details, $M=23.31 \mathrm{~s}, S D=6.67$, range $=5$ $-63 \mathrm{~s} ; M=20.45 \mathrm{~s}, S D=5.75$, range $4-71 \mathrm{~s} ; t(79)=4.63, p<.01$, Cohen's $d=0.52$.

\section{Exposure to Misinformation}

Subjects were exposed to between o and 4 misleading details, $M=2.73, S D=$ 1.07. In total they were exposed to misinformation on 436 out of 640 possible instances $(68 \%)$. Three subjects were exposed to misinformation for details that were intended to act as nondiscussed details (out of 640 possible instances; < $1 \%$ ); as in Experiment $2 \mathrm{~B}$, these details were excluded from the analyses.

\section{Remembering Discussed Details}

Consistent with the previous experiments, I next calculated subjects' proportion correct scores for discussed and nondiscussed details based on their 
responses on the final recognition test; these data are displayed in Figure 6.1.

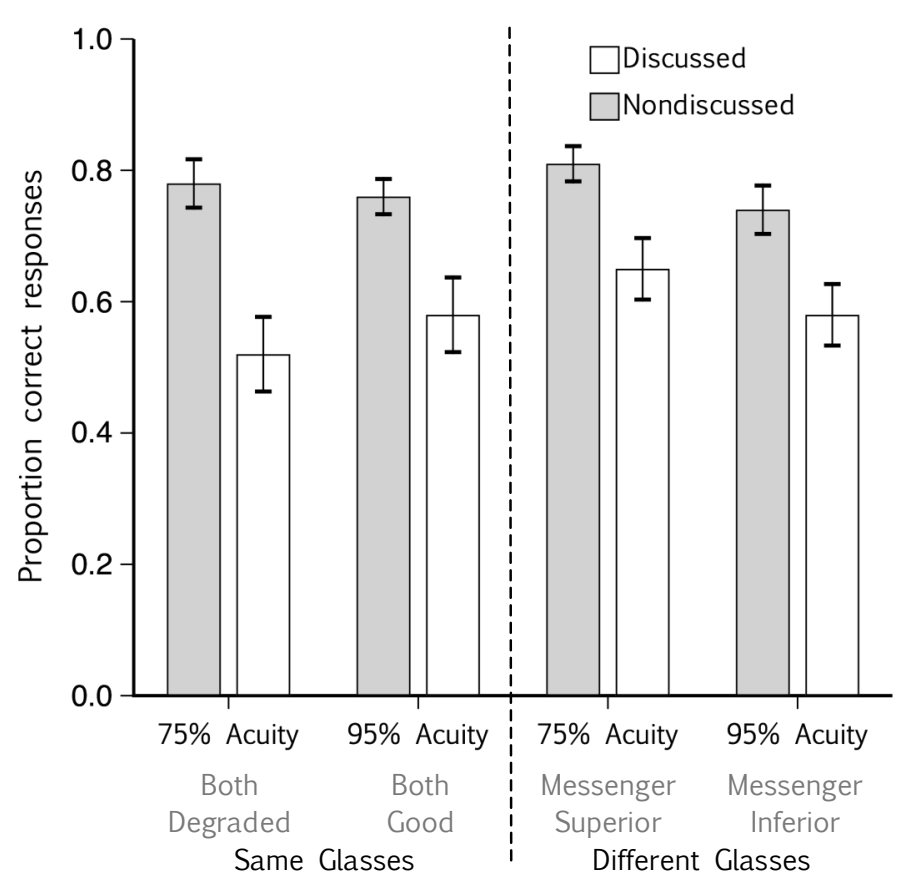

Figure 6.1. Proportion of correct responses for nondiscussed and discussed details by expectancy condition. Error bars depict standard errors of the means.

Did subjects' expectations about their own abilities and their expectations about their partner's abilities affect their susceptibility to misinformation? As Figure 6.1 illustrates, the answer is no. Subjects' memory performance was not affected by their expectations about their own or their partner's ability to see, $F<$ $1, n s .^{8}$ In fact, the only interesting finding from this analysis is that—consistent with the previous experiments-subjects in all conditions demonstrated a misinformation effect: they were better at remembering nondiscussed than discussed details, $F\left(1,15^{2}\right)=38.23, p<.01, f=0.50$.

Additionally, as displayed in Figure 6.2, the item-analysis showed that subjects remembered each critical detail better when they had not discussed it, $t(7)=7.94$, $p<.01$, Cohen's $d=2.81$.

\footnotetext{
${ }^{8}$ I repeated the analysis examining only discussed details. There were no differences between conditions-subjects' expectations did not affect their susceptibility to misinformation, $F<1, n s$.
} 


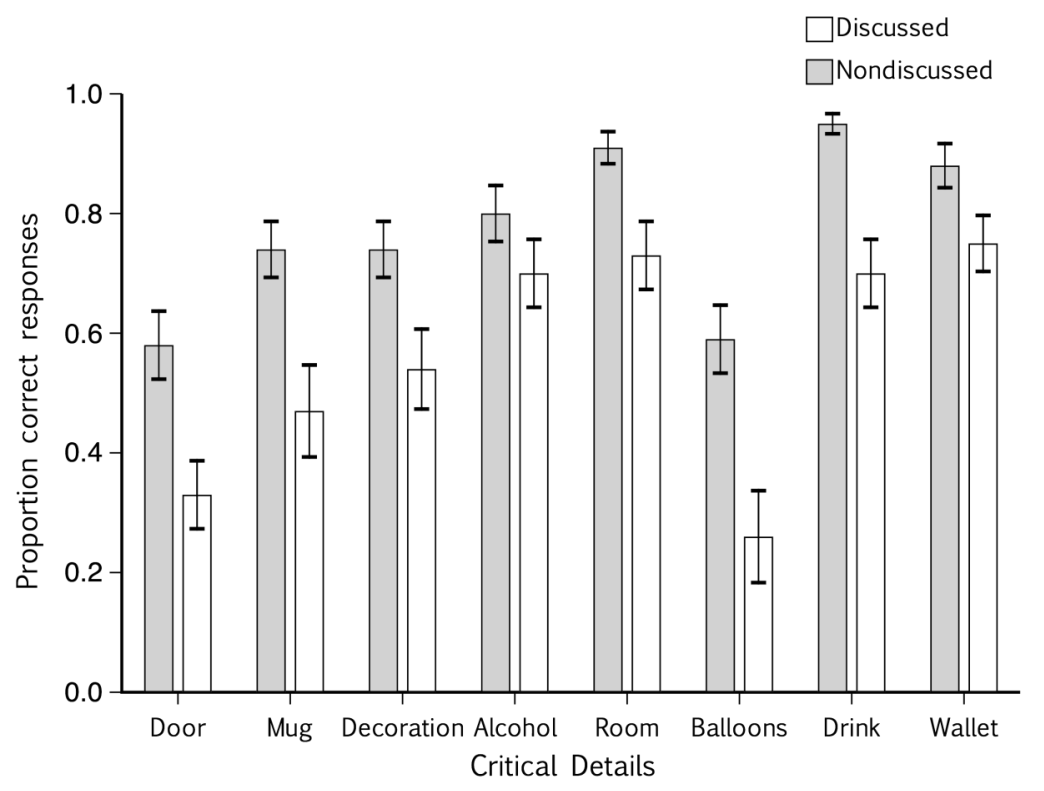

Figure 6.2. Proportion of correct nondiscussed and discussed responses for each critical detail. Error bars depict standard errors of the means.

Following the previous experiments, I next examined subjects who were exposed to at least two misleading details. Of these 139 subjects, $35(25 \%)$ were never misled by their partner's suggestions, $14(10 \%)$ were always misled by their partner's suggestions, and the remaining $90(65 \%)$ were misled about some details but resisted the suggestions about other details. These results suggest that people did make different judgments about the information conveyed-yet, their judgments did not appear to be driven by the manipulation. I grouped subjects based on their expectations of their own and their partner's abilities (bothdegraded; both-good; messenger-superior; messenger-inferior) according to whether they were never misled, sometimes misled or always misled; these data are displayed in Table 6.2. As Table 6.2 illustrates-and consistent with the primary analysis—patterns of responding were similar across the different conditions, providing more evidence that the manipulation did not affect subjects' susceptibility to misinformation. 
Table 6.2

Proportion of subjects who-when exposed to at least two misleading details-were never, sometimes, and always misled, by expectancy condition

PARTNER'S GLASSES

\begin{tabular}{lcccc} 
& \multicolumn{2}{c}{ Same } & \multicolumn{2}{c}{ Different } \\
\cline { 2 - 5 } SUBJECT'S GLASSES & $\begin{array}{c}75 \% \text { Acuity } \\
(\mathrm{n}=30) \\
\text { Both } \\
\text { Degraded }\end{array}$ & $\begin{array}{c}95 \% \text { Acuity } \\
(\mathrm{n}=34) \\
\text { Both } \\
\text { Good }\end{array}$ & $\begin{array}{c}75 \% \text { Acuity } \\
(\mathrm{n}=36) \\
\text { Messenger } \\
\text { Superior }\end{array}$ & $\begin{array}{c}95 \% \text { Acuity } \\
(\mathrm{n}=39)\end{array}$ \\
$\begin{array}{l}\text { Messenger } \\
\text { Inferior }\end{array}$ \\
Never Misled & $.20(6)$ & $.26(9)$ & $.28(10)$ & $.26(10)$ \\
Sometimes Misled & $.63(19)$ & $.65(22)$ & $.69(25)$ & $.61(24)$ \\
Always Misled & $.17(5)$ & $.09(3)$ & $.03(1)$ & $.13(5)$
\end{tabular}

There are several possible explanations for the results. It might be that neither expectations about the self nor expectations about the misinformation messenger affect susceptibility to false memories during a social interaction. Yet the research described above (for example, Gabbert et al., 2007; Skagerberg \& Wright, 2008) suggests that these kinds of judgments should have some effect. Recall that subjects rated only their own vision, not their beliefs about their partner's vision: an alternative explanation is that the manipulation did not affect subjects' expectations about their partner-the misinformation messenger-and thus did not affect their susceptibility to false memories. Another possibility is that—even though their memory performance was not affected—subjects in different conditions might have had different subjective experiences of their memories. For instance, subjects in the messenger-superior group might have been less confident about their memories because they thought their vision was degraded, or they might have been more confident when they were influenced by the superior messenger's suggestions. To address these possibilities, I next examined subjects' confidence in their responses. 


\section{Confidence}

Subjects' confidence ratings for their responses to discussed and nondiscussed details are displayed in Figure 6.3. As the figure illustrates, subjects' expectations about their own and their partner's abilities had no effect on their confidence, $F$ $(1,151)=1.10, p=.30$. Across conditions, subjects were moderately confident in all their responses.

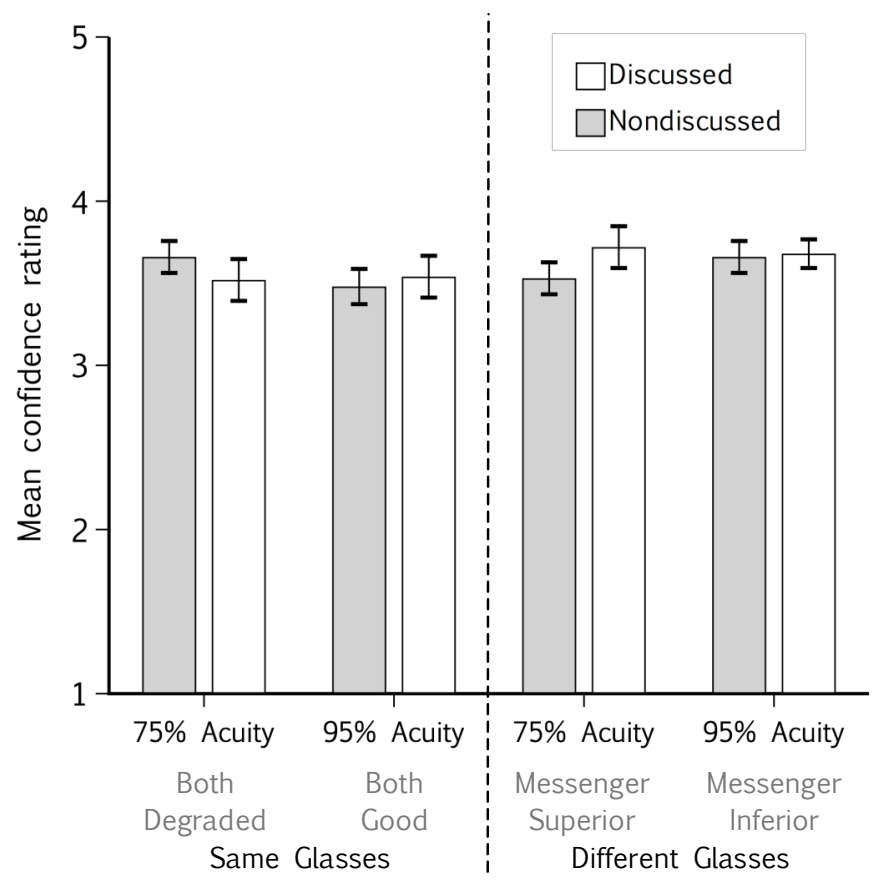

Figure 6.3. Mean confidence for nondiscussed and discussed details by expectancy condition. Error bars depict standard errors of the means.

I next examined subjects' confidence in their correct responses on nondiscussed details (true memories) and their incorrect responses on discussed details (false memories); these data appear in Figure 6.4. As the figure illustrates, subjects' expectations did not affect their confidence, $F<1, n s .{ }^{9}$ However, across conditions, subjects were more confident in their true than false memories, $F(1$, 107) $=30.04, p<.01, f=0.53$.

\footnotetext{
${ }^{9}$ I repeated the analysis examining only confidence in false memories-there were no differences in subjects' confidence based on their expectations, $F(3,110)=1.08, p=.36$.
} 


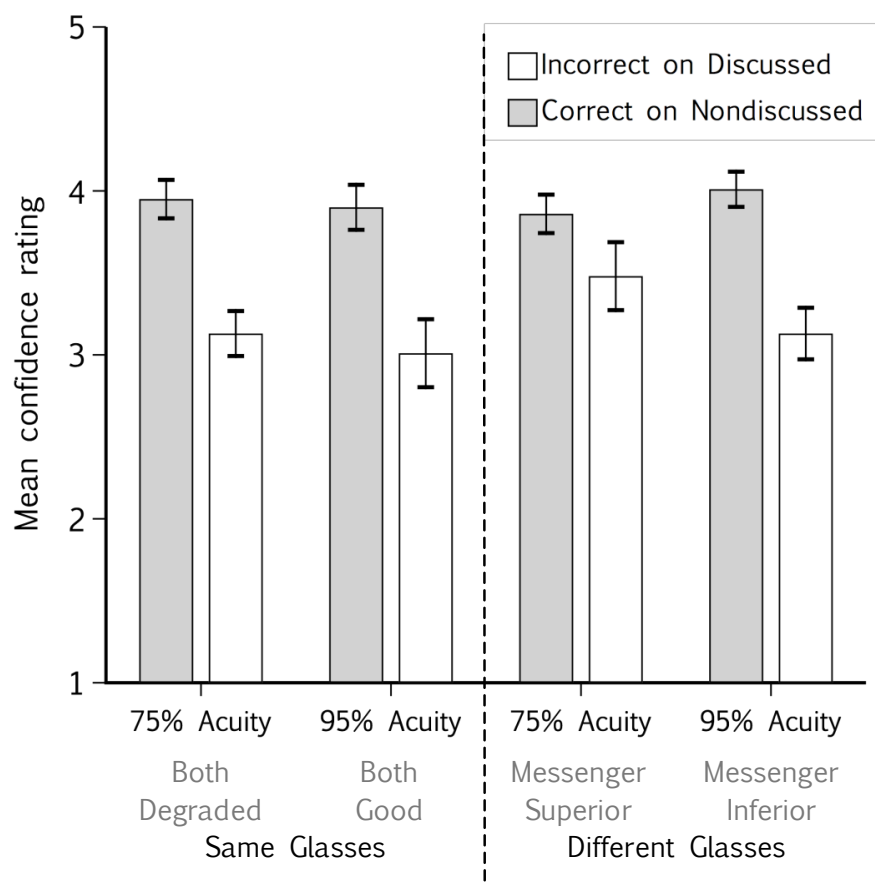

Figure 6.4. Mean confidence for correct responses on nondiscussed details (true memories) and incorrect responses on discussed details (false memories) by expectancy condition. Error bars depict standard errors of the means.

In short, there was no evidence that the manipulation affected subjects' memory performance or their subjective experiences of their memories, thus supporting the explanation that the manipulation may not have been effective at changing subjects' perceptions of their discussion partners.

\section{Features of the Discussion}

As in the previous experiments, I next examined the features of subjects' discussions to see if there were differences based on their expectations. I used the voicefiles and my written records of the discussions to determine whether each of four features occurred: whether the subject spoke first; whether the subject disputed his or her partner; whether the subject was disputed by his or her partner; and whether the subject used the misinformation as a discussion answer. Consistent with the previous experiments, for the most part, when one subject disputed the other, the other subject disputed back. In fact, there were only 50 
occasions (out of 436 possible instances; $11 \%$ ) where one subject disputed what the other said, and the other subject did not reciprocate, meaning that the two features were not different enough to analyse separately. Instead, as in the previous experiments, I examined only whether the subject disputed what his or her partner said. Focusing on the 436 instances where subjects were exposed to misinformation, I calculated the proportion of discussions where each feature occurred, and the subsequent proportion of instances where subjects went on to be misled. These data appear in Table 6.3. As the table illustrates, the discussion features occurred at similar rates, and subjects' patterns of responding were similar regardless of their expectations about their own and their partner's abilities.

Consistent with the previous experiments, the first two lines of Table 6.3 show that subjects were misled more often when they used the misinformation as their discussion answer than when they used another alternative. Yet using the misinformation as a discussion answer did not always lead subjects to be misled: sometimes they answered with the misinformation during discussion but reverted to the correct answer at test. Additionally, subjects occasionally used a different answer during the discussion, and then changed to the misinformation at test. 
Table 6.3

Proportion discussion feature occurrence and subsequent proportion of subjects misled for 436 instances that subjects were exposed to misinformation

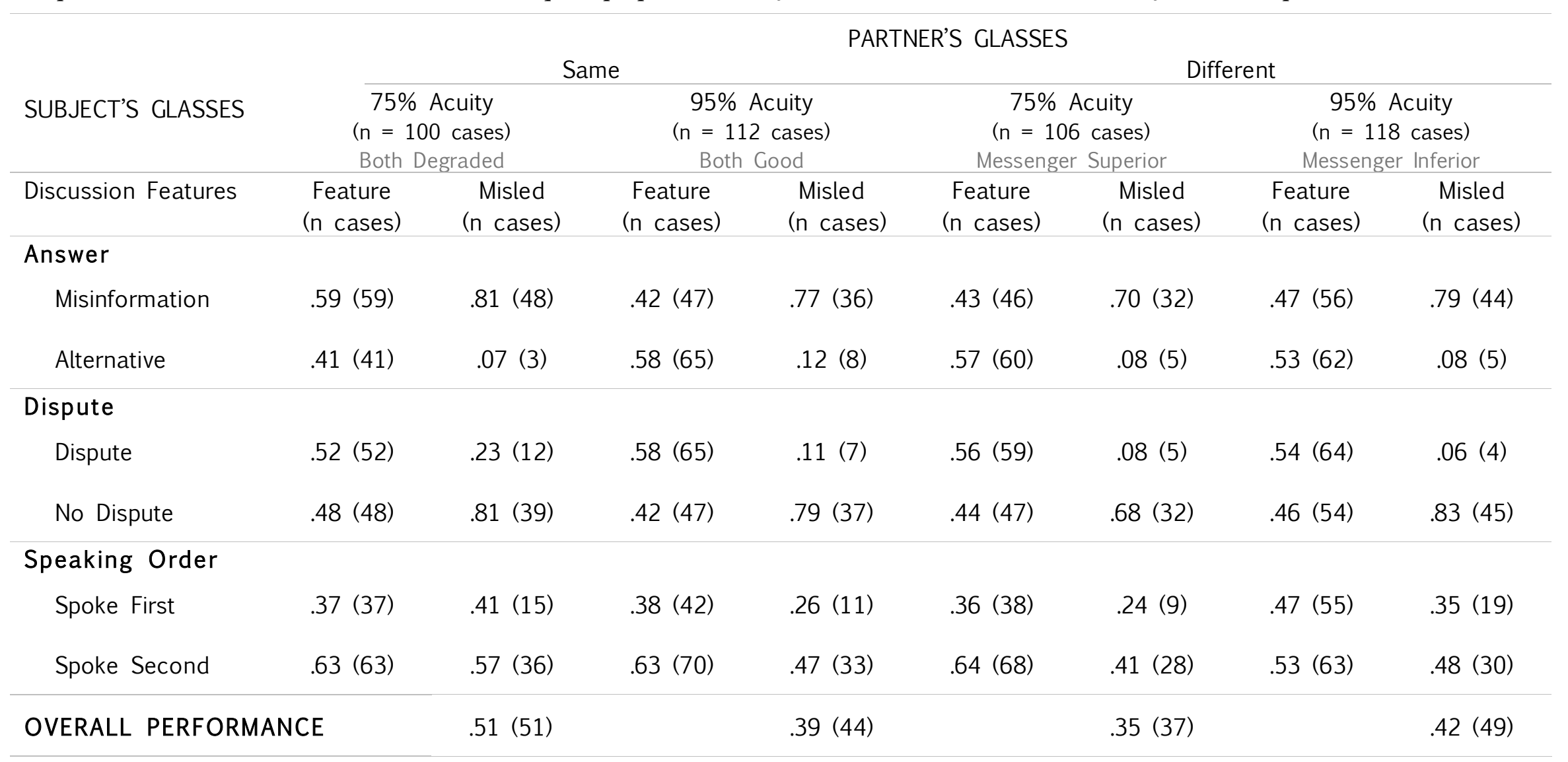

Note. Proportion Correct = 1 - Proportion Misled. For each condition ( $\mathrm{n}$ cases Correct $=$ total $\mathrm{n}$ cases $-\mathrm{n}$ cases Misled). 
Table 6.3 also shows that subjects were misled more often when they did not dispute what their partner said, and they were misled less often if they spoke first when discussing a critical detail. Additionally, when they were exposed to misinformation, subjects spoke first less often. However, this pattern was not true of subjects in one condition: subjects in the messenger-inferior group spoke first more often than subjects in the other three groups. Perhaps these subjects did believe they were superior to their partners, and as such spoke up first more often than subjects in other conditions. However, other than this one small difference, the patterns of responding were very similar to the previous experiments; the expectancy manipulation did not seem to affect subjects' discussion behaviour or their memory responses.

\section{Predicting False Memories}

Following the previous experiments, I next examined whether any discussion features predicted whether subjects were misled on a given detail. I conducted two nominal logistic regressions, by entering each of the three dichotomous discussion features as predictors. Additionally, I entered subjects' expectations about their own vision ( $75 \%$ or $95 \%$ acuity) and subjects' expectations about their partner's vision (same or different acuity) as predictors. In the first regressionthe all-items regression-I followed Gabbert et al.'s (2006) approach, and included all 640 instances where subjects were asked about a critical detail during the discussion, regardless of whether they were exposed to misinformation. In the second regression-the exposure-only regression-I addressed Lindsay's (2007) concerns about not correcting for exposure to misinformation by examining only the 436 instances where subjects were exposed to misinformation. Neither subjects' expectations about their own abilities nor their expectations about their partner's abilities predicted whether they were misled in either analysis. 
All-items regression. When I included all 640 instances that subjects were asked about a critical detail during the discussion, regardless of whether they were exposed to the misinformation, there were five predictors of being misled. First, subjects who used the misinformation as a discussion answer were nearly 16 times more likely to be misled than subjects who gave an alternative answer during the discussion, $\chi^{2}(1, N=640)=79.61, p<.01$. Second, subjects who disputed what their partner said were 7 times less likely to be misled than those who did not dispute their partner, $\chi^{2}(1, N=64 \mathrm{O})=41.22, p<.01$. Third, consistent with Gabbert et al. (2006), when exposure to misinformation was not taken into account, subjects who spoke first during the discussion of a critical detail were only half as likely to be misled as those who spoke second, $\chi^{2}(1, N=640)=8.21, p$ $<$.o1. Finally, there were two interactions: when subjects disputed what their partner said, they were twice as likely to be misled if they answered with the misinformation than if they answered with an alternative, $\chi^{2}(1, N=640)=6.39, p$ $=$. o1. Additionally, when subjects disputed what their partner said and used the misinformation as their discussion answer, they were only half as likely to be misled if they spoke first during the discussion of the detail, $\chi^{2}(1, N=640)=4.78$, $p=.03$.

Exposure-only regression. When examining only the 436 instances that subjects were exposed to misinformation, only two factors predicted being misled. First, subjects who used the misinformation as their discussion answer were 11 times more likely to be misled than subjects who used an alternative answer, $\chi^{2}(1, N=436)=59.14, p<.01$. Second, subjects who disputed what their partner said were 8 times less likely to be misled than those who did not dispute their partner, $\chi^{2}(1, N=436)=47.83, p<.01$.

Consistent with the previous experiments, speaking first only predicted being 
misled when exposure to misinformation was not taken into account, in the allitems regression. This result supports Lindsay's (2007) suggestion that speaking first is related to exposure to misinformation, but perhaps not susceptibility to false memories.

Predicting exposure regression. Finally, I conducted another regression, to see if any discussion features predicted whether subjects would be exposed to misinformation. Consistent with the previous experiments, subjects who disputed what their partner said were 19 times more likely to be exposed to misinformation, $\chi^{2}(1, N=640)=98.11, p<.01$. Additionally, in keeping with Lindsay's (2007) hypothesis, subjects who spoke first during the discussion of a critical detail were 5 times less likely to be exposed to misinformation, $\chi^{2}(1, N=$ $640)=64.35, p<.01$, thus protecting these subjects from being misled. These results provide more support for the idea that speaking order associated with exposure to misleading suggestions, but not necessarily susceptibility to misleading suggestions.

\section{Summary}

Taken as a whole, the results from this study fit with those of the previous experiments. Subjects remembered seeing details that they were merely told about during a discussion. Additionally, features within the interaction predicted whether subjects were more or less likely to be misled. However, the results do not shed any light on how people's expectations of themselves or their expectations of the misinformation messenger might operate to produce false memories in a social interaction. Yet other research suggests that people's expectations about themselves and about the misinformation messenger should affect false memories (for example, Assefi \& Garry, 2003; Clifasefi et al., 2007; Dodd \& Bradshaw, 1980; Echterhoff, Hirst, et al., 2005; Gabbert et al., 2006; Parker et al., 2008; Skagerberg \& Wright, 2008; Vornik et al., 2003). The best explanation 
of these null results is that the manipulation was not effective at changing people's perceptions of each other. My next goal was to improve the manipulation in the hope that I would be able to answer my original research question-whether people's expectations about themselves and their expectations about the misinformation messenger work in combination to determine their susceptibility to false memories. 


\section{Chapter 7}

\section{Experiment 6: Expecting the Best Protects People from False} Memories that Arise from Discussion

In Experiment 6, I made several methodological changes to cultivate subjects' expectations about their own and each other's abilities. I also set a minimum performance criterion on the filler tasks to ensure subjects at least attempted to do the tasks properly; if subjects made very little attempt on the tasks it would suggest that they were not motivated to do the tasks properly, thus decreasing the chance that they would consider the manipulation information, and decreasing the chance that they were concentrating enough to be sufficiently distracted during the filler tasks.

\section{Method}

\section{Subjects}

In total, 96 IPRP students took part in pairs in return for course credit.

\section{Design}

The design was identical to Experiment 5 .

\section{Materials and Procedure}

The procedure was identical to Experiment 5, except for five changes: [1] I changed the names of the acuity glasses; [2] extended the explanation of the glasses in the cover story; [3] added several preliminary manipulation tasks; [4] asked subjects to reiterate what type of glasses they wore immediately prior to the discussion; and [5] added a new manipulation check.

To make it clearer to subjects what the glasses were supposed to do, I changed the names of the acuity glasses. Instead of labeling the boxes, $35 \%, 55 \%, 75 \%$, and 95\% acuity (see Figure 2.1), I made new labels for "Poor”, "Moderate” and "Optimal" strength acuity glasses, each with a specified vision range (see Figure 7.1). Thus, instead of requiring subjects to remember what $75 \%$ and $95 \%$ glasses 
should do, they would know from the labels that they would have moderate or optimal vision.

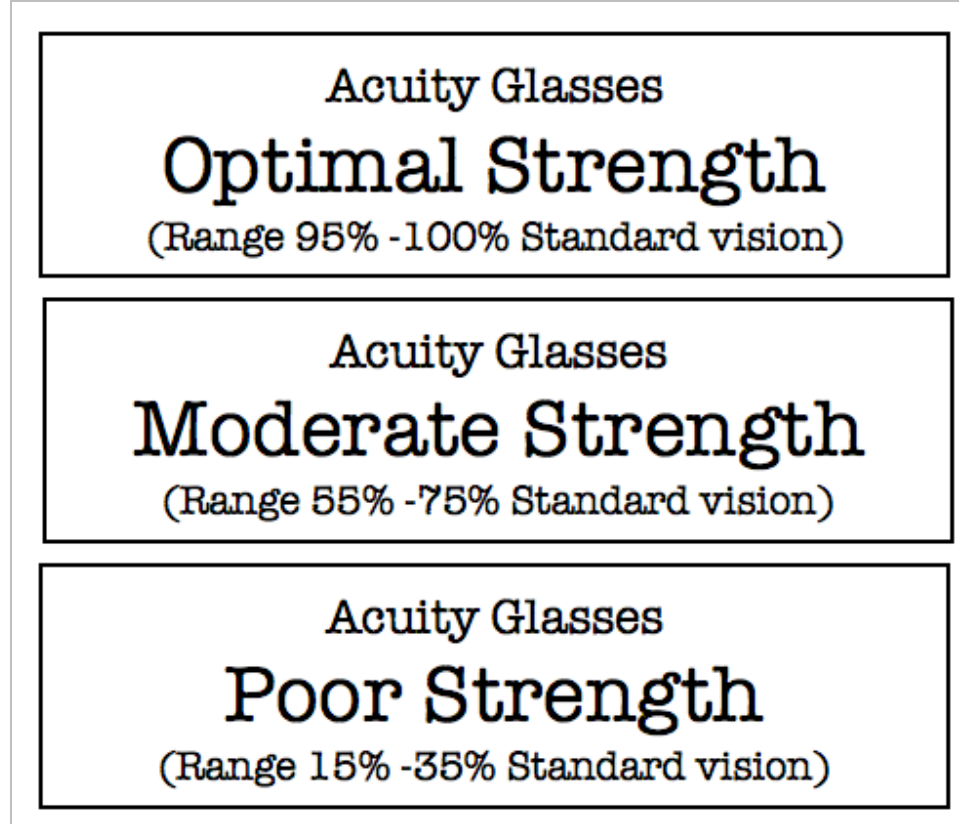

Figure 7.1. Updated box labels for different strengths of "Acuity Glasses.”

Additionally, I extended my explanation of the glasses in the cover story. Specifically, I said:

As you can see we have three different strengths of glasses; basically the higher the percentage range, the closer they are to normal vision. So the optimal glasses provide ideal viewing conditions-they are like the equivalent of normal vision. The other two types of glasses both degrade your vision to a different extent; both types are significantly worse than normal vision and the poor strength should be even harder to see through than the moderate strength glasses.

I wanted to ensure that subjects fully comprehended how the glasses would affect their ability to see; instead of just telling them and expecting them to believe me, I created several tasks so that subjects experienced normal and degraded vision. Because the glasses did not actually degrade subjects' vision, I used the MORI technique to show subjects different quality images through the 
different glasses. First, subjects were told:

We have a couple of different tasks today. I'm going to show you a few different images on this screen and get you to look at them through the acuity glasses. First I am going to compare what you can see with the optimal glasses, and what you can see with the moderate glasses. You'll each have a turn at wearing each type of glasses.

Subjects each chose a card that told them what sort of glasses they would wear: one person was given glasses from the optimal box, and the other was given glasses from the moderate box. There were two tasks in the manipulation phase of the study, a letter array and a face recognition task. For each task, I created two different images, a clear image, and a degraded image. I used the MORI technique to present the poorer quality image to the subject who wore moderate glasses and the better quality image to the subject who wore optimal glasses, thus giving subjects the impression that the glasses affected their ability to see.

Letter array. In the first task, subjects saw a six-line letter array, similar in appearance to an optician's array. To create the degraded array, I used a lighter colour font, and increased the transparency of the letters so they would fade into the background (see Figure 7.2). I designed the arrays so that when subjects saw the normal version they could generally read to the end of the sixth line and when they saw the degraded version they could generally read only to the end of the fourth line. Subjects took turns to read the letters that they could see out loud, then swapped glasses, and repeated the task. As a result, subjects had the experience of better and worse vision from the different glasses, the experience of seeing letters that their partner could not see, and the experience of not being able to see letters that their partner could see. 

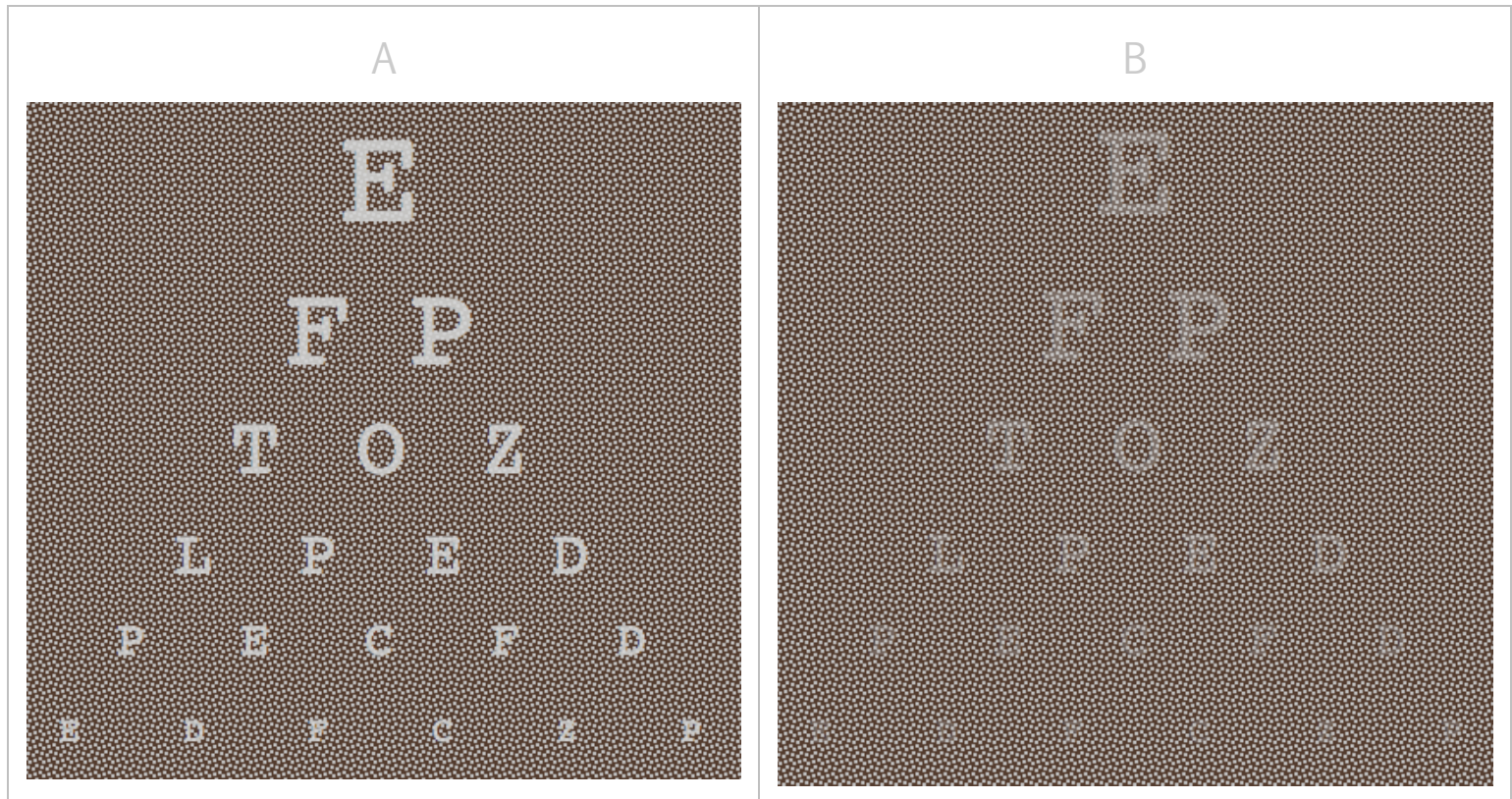

Figure 7.2. Arrays used for letter recognition task. Subjects wearing optimal glasses saw clear array $[\mathrm{A}]$ and subjects wearing moderate glasses saw degraded array $[\mathrm{B}]$.

Face recognition task. In the face recognition task, I presented four photographs of famous people-one at a time-for 2 seconds each. I created a second degraded version of each photo by adding grain and blur in Adobe Photoshop® (see Figure 7.3). Typically subjects could recognise the normal photos very easily and confidently, but had much more difficulty with the degraded photos and often had no idea who these photographs depicted. After each photograph was presented, subjects were asked to report (out loud) whether they recognised the face, and if they did, how confident they were. After both subjects had answered these questions, each was asked who the photograph depicted. As before, subjects who wore moderate glasses saw the degraded photographs, and subjects who wore optimal glasses saw normal photographs. After two photographs, subjects swapped glasses so that each saw two degraded faces and two normal faces. 


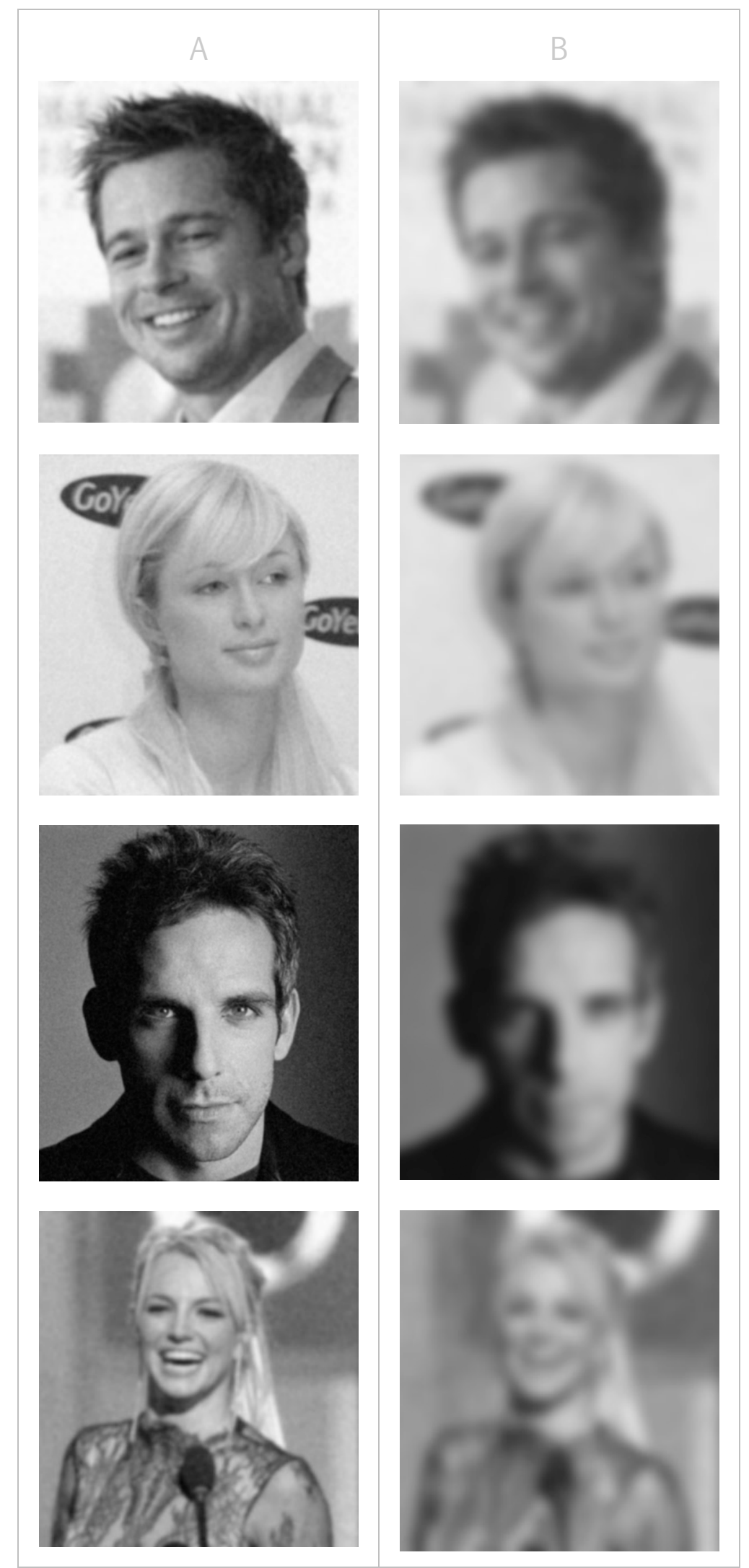

Figure 7.3. Celebrity photographs used for face recognition task. Subjects wearing optimal glasses saw clear photos $[\mathrm{A}]$ and subjects wearing moderate glasses saw degraded photos $[\mathrm{B}]$. From the top, the photos depict Brad Pitt, Paris Hilton, Ben Stiller and Britney Spears. 
Following the preliminary tasks, subjects were asked to give their glasses back, and were then told:

The next thing I am going to do is show you a movie. The movie is about a guy named Chad who is at a party. All I want you to do is watch the movie through the acuity glasses, and then we will do a couple of related tasks afterwards. Its just like the other tasks-people see best when they keep their head straight rather than tilted, so as with the previous tasks I'll ask you to keep your heads as straight and still as possible while you watch the movie. Because the movie takes longer than the tasks we have just done, for this part of the study you are only going to be able to wear one pair of glasses each. You each have an envelope in front of you; each envelope has three cards inside-one card for each type of acuity glasses. If you reach into those envelopes now and take out a card it will tell you what condition you'll be in for the movie today.

Each subject was given a pair of glasses from either the moderate or optimal box and watched the Chad movie as in the previous studies.

I wanted to ensure that subjects remembered what type of glasses they wore and what type of glasses their partner wore when they discussed the movie. As a reminder, at the beginning of the discussion when the voice recorder had been started, subjects stated where they were sitting (left or right) and the type of glasses that they wore during the movie, under the guise that it would help me distinguish who said what in the voicefile.

Finally, I added a new manipulation check. As in Experiment 5, subjects rated their vision and sensory compensation. However, before reporting which glasses they wore and which glasses their partner wore, subjects filled out another fouritem manipulation check. These four self-partner comparison measures asked subjects to rate how they compared to their partner in their ability to see the movie, their ability to make out the details, their credibility and their reliability; subjects responded on separate 7-point Likert scales ranging from -3 (partner better) to +3 (self better), where the midpoint $\mathrm{o}=$ "about the same." 
Results and Discussion

To ensure that subjects were sufficiently distracted from thinking about the critical material during filler tasks, and to ensure that they were motivated enough to care about the preliminary manipulation tasks, I set a minimum performance criterion for the filler tasks. To be included in the dataset, subjects were required to attempt at least five squares during the 10 min of suduko puzzles, and find at least three words during the 5 min wordfind. In total 48 pairs completed the study and met this filler task criterion. ${ }^{10}$

As in the previous experiments, all data was analysed on an individual level, yielding a total of 96 subjects. Subjects attempted between 6 and 84 squares on the suduko puzzle, $M=26.02, S D=13.97$, and found between 4 and 16 words in the wordfind, $M=7.66, S D=2.66$. Consistent with the previous experiments, subjects were good at remembering the 12 filler details, and it made no difference whether they wore moderate or optimal glasses, $M=8.69$ correct $(72 \%), S D=1.17$; $M=8.77 \operatorname{correct}(73 \%), S D=1.43 ; t<1, n s$.

\section{Manipulation Checks}

Did the manipulation affect subjects' expectations of their own abilities? Consistent with Experiment 5, subjects who wore moderate glasses rated their vision as significantly worse than subjects who wore optimal glasses, suggesting that people's perceptions of their viewing ability were driven by the manipulation, $M=2.51, S D=0.52 ; M=3.67, S D=0.69 ; t(94)=6.87, p<.01$, Cohen's $d=1.90$. Additionally, subjects who wore moderate glasses were marginally more likely to report higher sensory compensation than subjects who wore optimal

\footnotetext{
${ }^{10}$ Nine subject-pairs were excluded from the dataset because one person in each pair did not make a sufficient attempt on the filler tasks. Of these, five people attempted less than 5 squares during the 10 min suduko puzzle, and four people found less than 3 words during the 5 min wordfind.

An additional three pairs were excluded because one person in each pair expressed suspicion that they might have seen different movies.
} 
glasses, suggesting that they thought their vision was actually degraded, $M=$ 2.50, $S D=1.17 ; M=2.06, S D=1.10 ; t(94)=1.89, p=.06$, Cohen's $d=0.39$.

I next examined whether the manipulation affected subjects' perceptions of their partner's abilities. For each subject I calculated a mean score based on their four self-partner comparison measures; these data are displayed on Figure 7.4. As Figure 7.4 illustrates, subject-pairs who wore the same glasses as each other rated themselves as very similar, regardless of whether they were in the both-degraded group or the both-good group; subjects in the messenger-superior group rated their partners as better; and subjects in the messenger-inferior group rated themselves as better. In short there was an interaction between subjects' expectations of themselves and their expectations of their partner, $F(1,92)=$ $49.89, p<.01, f=0.74$. Taken as a whole, these results suggest that the credibility manipulation led subjects to believe that glasses affected their ability to see and their partner's ability to see.

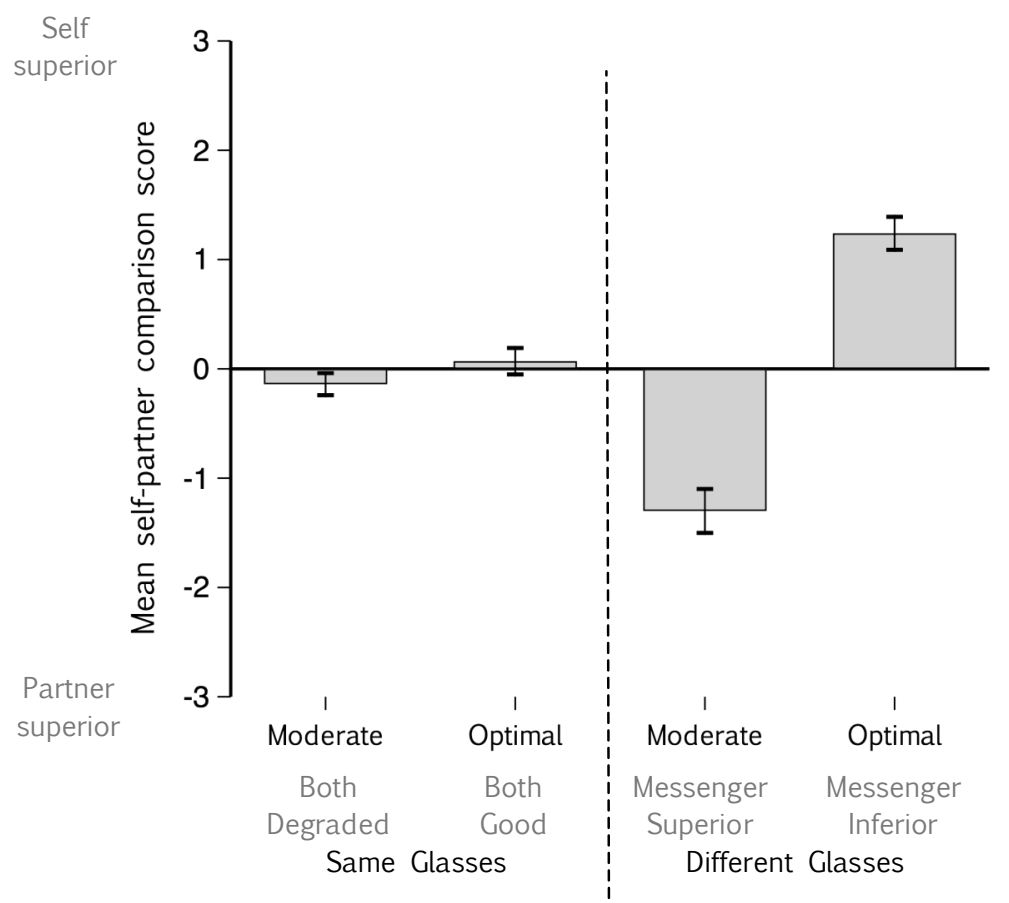

Figure 7.4. Mean self-partner comparison ratings by expectancy condition. Error bars depict standard errors of the means. 


\section{Discussion Duration}

As in the previous experiments, there was no evidence that subjects felt rushed to select their answers during the discussion; subject pairs took between 2:05 min and 6:02 min to complete the discussion, $M=3: 52 \mathrm{~min}, S D=0: 55$. Interestingly, subject-pairs who wore the same glasses took less time to discuss the questions than subject-pairs who wore different glasses, $M=3: 33 \mathrm{~min}, S D=$ o:48; $M=4: 12 \min , S D=0: 56 ; t(46)=2.59, p=.01$, Cohen's $d=0.75$. This difference was driven by both critical and filler details; pairs who wore different glasses took longer than pairs who wore the same glasses to discuss both critical details- $M=23.97 \mathrm{~s}, S D=7.64 ; M=19.75 \mathrm{~s}, S D=5.83$ - and filler details- $M=19.46$ sec, $S D=5.16 ; M=16.69 \mathrm{~s}, S D=4.35 ; F(1,46)=6.87, p=.01, f=0.10$. Perhaps pairs who wore different glasses were less trusting of each other, or were curious about each other's experience, thus extending their discussion time. Across all conditions, subjects took longer to discuss critical details than filler details, $M=$ $21.86 \mathrm{~s}, S D=7.05$, range $=5-65 \mathrm{~s} ; M=18.08 \mathrm{~s}, S D=4.93$, range $=4-68 \mathrm{~s} ; F(1,46)=$ 13.04, $p<.01, f=0.53$.

Pairs were given a warning when they had 10 sec remaining to discuss a given question. In total, pairs who wore different glasses were given 3 warnings when discussing filler details (out of a possible 192 instances; $2 \%$ ) and 2 warnings when discussing critical details (out of a possible 96 instances; $2 \%$ ). Of pairs who wore the same glasses only one was given a warning when discussing a filler detail (out of a possible 192 instances; < 1\%), and two were given warnings when discussing critical details (out of a possible 96 instances; $2 \%$ ). Taken together, these results suggest that people were not rushed during the discussion phase of the study.

\section{Exposure to Misinformation}

Subjects were exposed to between o and 4 misleading details, $M=2.63, S D=$ 
1.02; in total they were exposed to misleading details on 253 out of a possible 384 instances $(66 \%)$. Three people were exposed to misinformation for details that were intended to act as nondiscussed details; these details were excluded from the analyses. As in the previous experiments, I calculated-for each subject-a proportion-correct score for discussed and nondiscussed details.

\section{Remembering Discussed Details}

Recall that people's expectations might affect their susceptibility to false memories in three different ways. First, if susceptibility to false memories is driven by people's expectations about the misinformation messenger, then people who are told their discussion partner has optimal ability to see the movie should be more misled than people who are told their discussion partner has moderate ability, regardless of what they are told about their own abilities. Second, if susceptibility to false memories is driven by people's expectations about themselves, then people who are told they have moderate ability to see the movie should be more misled than people who are told they have optimal ability, regardless of what they are told about their partner's abilities. Finally, if susceptibility to false memories is affected by people's beliefs about themselves in combination with their beliefs about the misinformation messenger, we should see an interaction between the two factors. Additionally, any differences could be driven by some subjects being more susceptible to false memories, some subjects being less susceptible to false memories, or a combination of the two.

I compared subjects' proportion correct scores based on their expectations about their own and their partner's vision; these data are displayed on Figure 7.5. As the figure illustrates, subjects' expectations about their own abilities interacted with their expectations about their partner's abilities to determine how misled they were by their partner's suggestions, $F(1,89)=4.26, p=.04, f=0.22$. 
Pairs who wore the same type of glasses as each other showed the typical misinformation effect, and were better at remembering nondiscussed details than discussed details. In other words, on the final test they incorrectly reported details that they were told about during the discussion, but never actually saw. These subjects were just as influenced by their partner's suggestions whether they were in the both-degraded or the both-good group. However, when subjectpairs wore different glasses these patterns were quite different. Specifically, subjects in the messenger-superior group were influenced by their partner's suggestions, but subjects in the messenger-inferior group were not.

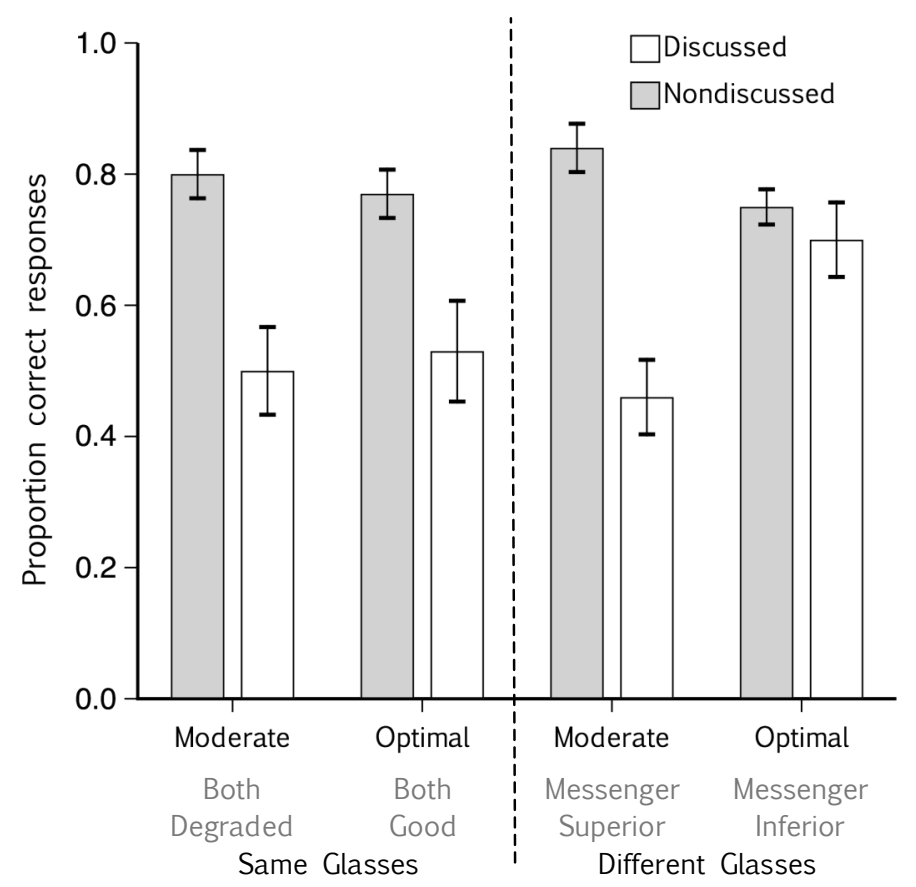

Figure 7.5. Proportion of correct responses for nondiscussed and discussed details by expectancy condition. Error bars depict standard errors of the means.

Interestingly, regardless of whether the pairs wore the same or different glasses, subjects who wore moderate glasses were better at remembering nondiscussed details than subjects who wore optimal glasses, $t(94)=1.63, p=.05$, Cohen's $d=0.32$. In other words, subjects who were told their vision was degraded 
actually had better baseline memories of the critical details. Perhaps these people exerted more effort during the movie, thus boosting their performance. However, this advantage in memory accuracy did not translate to decreased false memories.

In fact, subjects who wore moderate glasses did not experience any protection from false memories. As Figure 7.5 shows, subjects in the both-degraded group were only as accurate as their optimal counterparts—-the both-good group-at remembering discussed details, $t<1, n s$, and subjects in the messenger-superior group were actually worse than their optimal counterparts-the messengerinferior group—at remembering discussed details, $t(46)=2.82, p<.01$, Cohen's $d$ $=0.84$.

Recall that the differences in false memories between the messenger-superior group and the messenger-inferior group might be driven by one group experiencing increased susceptibility to misinformation, the other group experiencing decreased susceptibility to misinformation, or a combination of the two. To investigate these possibilities I generated a grand mean of baseline susceptibility to misinformation; the grand mean was based on subject-pairs who wore the same glasses, collapsed across subjects in the both-degraded group and subjects in the both-good group. I examined whether the messenger-inferior and messenger-superior groups were more or less influenced by misinformation by comparing their accuracy on discussed details with the grand mean baseline measure. Subjects in the messenger-superior group were no worse at remembering discussed details than the baseline measure, suggesting that these subjects did not experience increased susceptibility to misinformation, $M=.46$, $S D=.27 ; M=.51, S D=.35 ; t<1, n s$. However, subjects in the messenger-inferior group were significantly better at remembering discussed details than the baseline measure, suggesting that they experienced decreased susceptibility to 
misinformation, $M=.70, S D=.30 ; M=.51 ; S D=.35 ; t(68)=2.15, p=.04$, Cohen's $d$ $=0.58$. In fact, as Figure 7.5 shows, there was no misinformation effect for these subjects: they were just as good at remembering nondiscussed and discussed details, providing evidence that they were not influenced by the messenger's misleading suggestions at all, $t<1, n s$. Put another way, subjects in the messenger-inferior group were protected from the influence of misleading suggestions. Instead of subjects in the messenger-superior group experiencing increased susceptibility to misinformation, the difference in false memories was actually driven by subjects in the messenger-inferior group who experienced decreased susceptibility to misinformation.

However, recall that subjects who wore moderate glasses were better than subjects who wore optimal glasses at remembering nondiscussed details; in other words, subjects who wore moderate glasses had better baseline memories of the critical details. Perhaps subjects in the messenger-superior group were more susceptible to misinformation, but because the analysis did not take their superior baseline memories into account, this effect was hidden. Another way to examine susceptibility to misinformation is to compare the difference between subjects' correct responses for nondiscussed details and their correct responses for discussed details; the bigger the difference between these two scores, the greater the influence of the misinformation. If subjects in the messengersuperior group did experience increased susceptibility to misinformation, we should see bigger differences between their nondiscussed and discussed performance than we see for the other groups.

To address this possibility, I calculated another grand mean based on subjects who wore the same glasses as each other, collapsing across the both-degraded and the both-good groups; this grand mean measured the difference between subjects' accuracy for nondiscussed details and their accuracy for discussed 
details. To examine whether subjects in the messenger-superior and messengerinferior groups had bigger or smaller difference scores than baseline, I compared each to the grand mean difference. In fact, there was a trend that subjects in the messenger-superior group had greater differences between their nondiscussed and discussed scores than the grand mean, supporting the idea that these subjects were more susceptible to misinformation, $M=.40, S D=.33 ; M=.27$, $S D=.37 ; t(68)=1.37, p=.09$, Cohen's $d=0.36$. Additionally—consistent with the primary analysis—subjects in the messenger-inferior group had smaller differences between their nondiscussed and discussed performance than the grand mean, showing that they were less susceptible to misinformation, $M=.04$, $S D=.31 ; M=.27, S D=.37 ; t(68)=2.64, p<.01$, Cohen's $d=0.63$. In sum, subjects in the messenger-inferior group were less influenced by misinformation, and subjects in the messenger-superior group were more influenced by misinformation, suggesting that both factors probably contributed to the differences in false memories between these groups.

Taken as a whole, the results fit with the idea that people's judgments about the misinformation messenger-in this case their discussion partner-do not work in isolation. When subjects were told they had the same abilities as the messenger, they were equally misled by a messenger with apparently optimal ability and a messenger with apparently moderate ability. Yet, when subjects were told that their abilities were inferior to the messenger's abilities they were more susceptible to the messenger's misleading suggestions, and when subjects were told that their abilities were superior to the messenger's abilities they were not susceptible to the messengers' suggestions at all. These results build on Gabbert et al. (2007) and Skagerberg and Wright (2008): subjects in the supposedly disadvantaged role were more susceptible to false memories, but subjects in the supposedly advantaged role were also protected from false 
memories. Additionally, these differences were not by driven by subjects' perceptions of themselves, or their perceptions of the messenger, but by the two in combination.

Correcting for exposure to misinformation. One caveat with correcting for exposure to misinformation is that subjects in one condition were exposed to more misleading details than subjects in the other three conditions. Specifically, subjects in the messenger-superior group were exposed to 74 misleading details (out of a possible $96 ; 77 \%$ ) whereas subjects in the other three groups were only exposed to between 57 and 61 misleading details ( $59 \%$ to $64 \%$ ). This result is consistent with Gabbert et al.'s (2007) study, and suggests that—regardless of susceptibility to false memories - people who are seen as inferior might have a greater chance of being influenced by misinformation simply because they might come into contact with more bad information from other people who think they know better. Naturally, being exposed to more misinformation means that people have more opportunity to be misled; however, my calculations eliminated any contributing effects of exposure to misinformation. To account for this problem, I repeated the major analysis without correcting for exposure. In other words, I completed a second analysis, examining people's memories for the four critical details that were asked about during the discussion, regardless of whether they were exposed to misinformation when discussing those details.

I found exactly the same pattern of results as those displayed in Figure 7.5; subjects' expectations about their own abilities and about the messenger's abilities affected their susceptibility to false memories, $F(1,92)=5.20, p=.03, f=$ o.24. Additionally, follow up t-tests confirmed the same patterns of results. Subjects in the messenger-superior condition were just as accurate at remembering discussed details as the grand mean baseline measure-based on subjects in the both-degraded and both-good groups, $M=.56, S D=.24 ; M=.64, S D$ 
$=.27 ; t(70)=1.20, p=.24$, but subjects in the messenger-inferior group were more accurate than the baseline measure, $M=.80, S D=.21 ; M=.64, S D=.27 ; t(70)=$ 2.55, $p=.01$, Cohen's $d=0.61$. Additionally, subjects in the messenger-inferior group were not only not misled by their partner's suggestions, they were actually better at remembering discussed than nondiscussed details, providing more evidence that these subjects resisted the messenger's suggestions, $M=.80, S D=$ $.21 ; M=.73, S D=.16 ; t(23)=1.66, p=.05$, Cohen's $d=0.33$.

I next calculated the difference between subjects' correct responses for nondiscussed details and their correct responses for discussed details. As before, I compared subjects in the messenger-inferior group, and subjects in the messenger-superior group to the grand mean difference score, to see if they were any more or less susceptible to misinformation than the baseline measure. The results were consistent with the previous findings. Specifically, subjects in the messenger-superior group had bigger difference scores than the grand mean, suggesting that they experienced increased susceptibility to misinformation, $M=$ $.28, S D=.35 ; M=.15, S D=.33 ; t(70)=1.60, p=.06$, Cohen's $d=0.38$, and subjects in the messenger-inferior group had smaller difference scores than the grand mean, suggesting that they experienced decreased susceptibility to misinformation, $M=-.07, S D=.21 ; M=.15, S D=.33 ; t(70)=2.91, p<.01$, Cohen's $d$ $=0.70$. In summary, whether or not exposure to misinformation was accounted for, the results were very similar. Subjects' susceptibility to misinformation depended on their expectations of themselves and their expectations of the misinformation messenger.

Following the previous experiments, I next conducted an item-analysis to examine the contribution of each critical detail when acting as a nondiscussed and discussed detail. These data are displayed in Figure 7.6. As the figure illustrates, and consistent with the previous experiments, subjects remembered 
each critical detail better when they had not discussed it, $t(7)=8.81, p<.01$, Cohen's $d=3.11$.

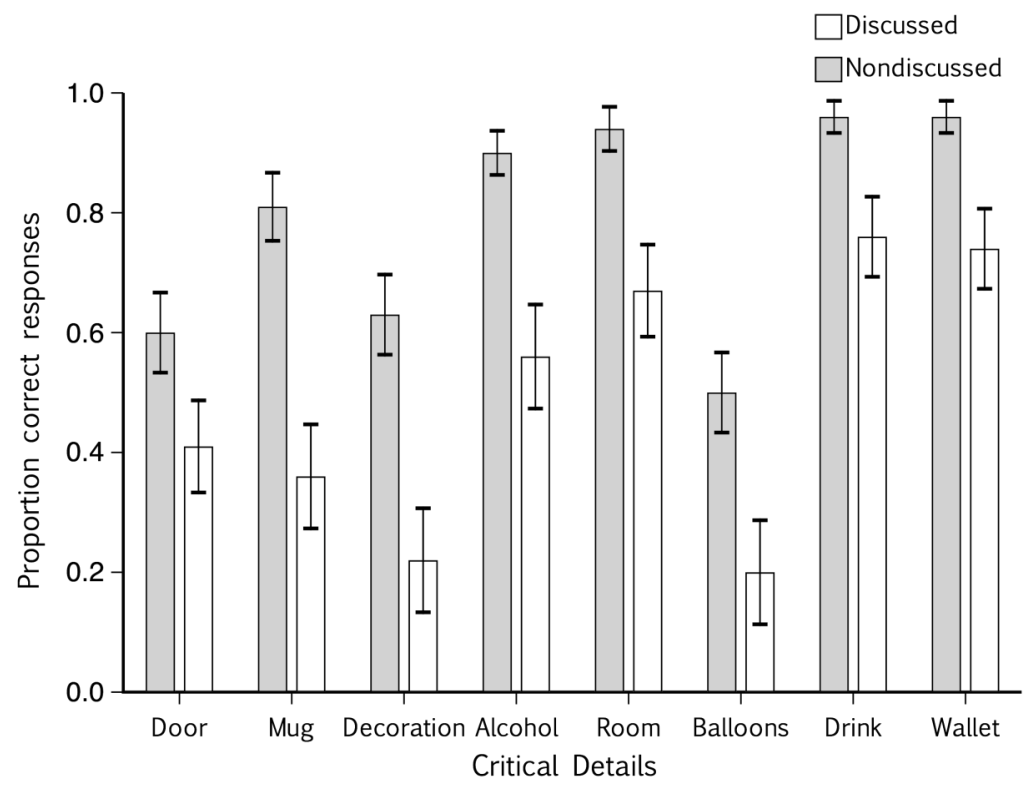

Figure 7.6. Proportion of correct nondiscussed and discussed responses for each critical detail. Error bars depict standard errors of the means.

As in the previous experiments, I next focused on subjects who were exposed to at least two pieces of misinformation. Of these 83 subjects, $10(12 \%)$ were misled by everything their partner told them, $14(17 \%)$ were not misled by anything their partner told them, and the remaining 59 (71\%) were misled about some details but not others. As in Experiment 5, I grouped subjects based on their expectations of their own and their partner's abilities (both-degraded; bothgood; messenger-superior; messenger-inferior) according to whether they were never misled, sometimes misled, or always misled; these data appear in Table 7.1.

What is most interesting about this table is the different ways that subjects responded. Although the manipulation largely protected subjects in the messenger-inferior group from being misled, there was still variation within the conditions. For instance, one subject in the messenger-inferior group was still misled by all of the messenger's suggestions. Additionally, two subjects in the 
messenger-superior group were never misled by any of the messenger's suggestions. In short, these results suggest that although people's judgments about their own abilities and about the misinformation messenger's abilities are important in determining their susceptibility to false memories, there are other factors at play as well. These factors might include judgments that subjects made about each other beyond the manipulation, features of the interaction that I did not manipulate or measure, and even personality differences, such as how confident or extraverted people are (Asch, 1952; 1953; 1966).

Table 7.1

Proportion of subjects who-when exposed to at least two misleading details - were never, sometimes, and always misled, by expectancy condition

\begin{tabular}{lcccc}
\hline & \multicolumn{3}{c}{ PARTNER'S GLASSES } \\
SUBJECT'S GLASSES & $\begin{array}{c}\text { Moderate } \\
(\mathrm{n}=20) \\
\text { Both } \\
\text { Degraded }\end{array}$ & $\begin{array}{c}\text { Optimal } \\
(\mathrm{n}=21) \\
\text { Both } \\
\text { Good }\end{array}$ & $\begin{array}{c}\text { Moderate } \\
(\mathrm{n}=23) \\
\text { Messenger } \\
\text { Superior }\end{array}$ & $\begin{array}{c}\text { Optimal } \\
(\mathrm{n}=19) \\
\text { Messenger } \\
\text { Inferior }\end{array}$ \\
\hline Never Misled & $.10(2)$ & $.24(5)$ & $.09(2)$ & $.26(5)$ \\
Sometimes Misled & $.80(16)$ & $.52(11)$ & $.82(19)$ & $.69(13)$ \\
Always Misled & $.10(2)$ & $.24(5)$ & $.09(2)$ & $.05(1)$
\end{tabular}

One puzzling result is that subjects who were told that they had degraded vision were actually better at remembering nondiscussed details, yet they were not protected from being misled. Given that their baseline memories were superior, these subjects should have been better able to notice the discrepancies between their own memories and the misleading suggestions, and thus better able to resist the influence of those suggestions (Tousignant, Hall, \& Loftus, 1986). Even though these subjects' memories were better, perhaps their subjective 
experiences of their memories were different, and somehow caused them to be influenced by — rather than protected from - their partner's misleading suggestions. To address this possibility, I next examined subjects' confidence in their responses.

\section{Confidence}

I first calculated how confident subjects were in their responses to nondiscussed and discussed details, and compared these ratings based on subjects' expectations about their own and their partner's vision; these data are displayed in Figure 7.7. As the figure illustrates, subject-pairs who wore the same glasses as each other-the both-degraded and both-good groups-were equally confident in their memories of discussed and nondiscussed details, but subjectpairs who wore different glasses-the messenger-superior and messengerinferior groups-were more confident in their memories of nondiscussed details than their memories of discussed details, $F(1,89)=4.72, p=.03, f=0.23$. This result suggests that pairs who wore different glasses may not have trusted each other: when they discussed critical details, their confidence dropped.

Additionally subjects who wore optimal glasses tended to be more confident in all their memories than subjects who wore moderate glasses, $F(1,89)=3 \cdot 33, p$ $=.07, f=0.10$. As Figure 7.7 shows, this effect was driven by subjects-pairs who wore different glasses. Follow up t-tests showed that subjects in the messengersuperior group tended to be less confident than subjects in the messengerinferior group in their responses to both nondiscussed details, $t(46)=2.08, p=$ .04, Cohen's $d=0.60$, and discussed details, $t(44)=1.72, p=.09$, Cohen's $d=1.46$. These results suggest a possible mechanism by which the people who had better memories ended up being more misled: even though their memories were better, subjects in the messenger-superior group had less confidence in their memories, and so were more susceptible to the influence of the messenger's 
suggestions. In short, having a good memory might not be sufficient to protect people from being misled.

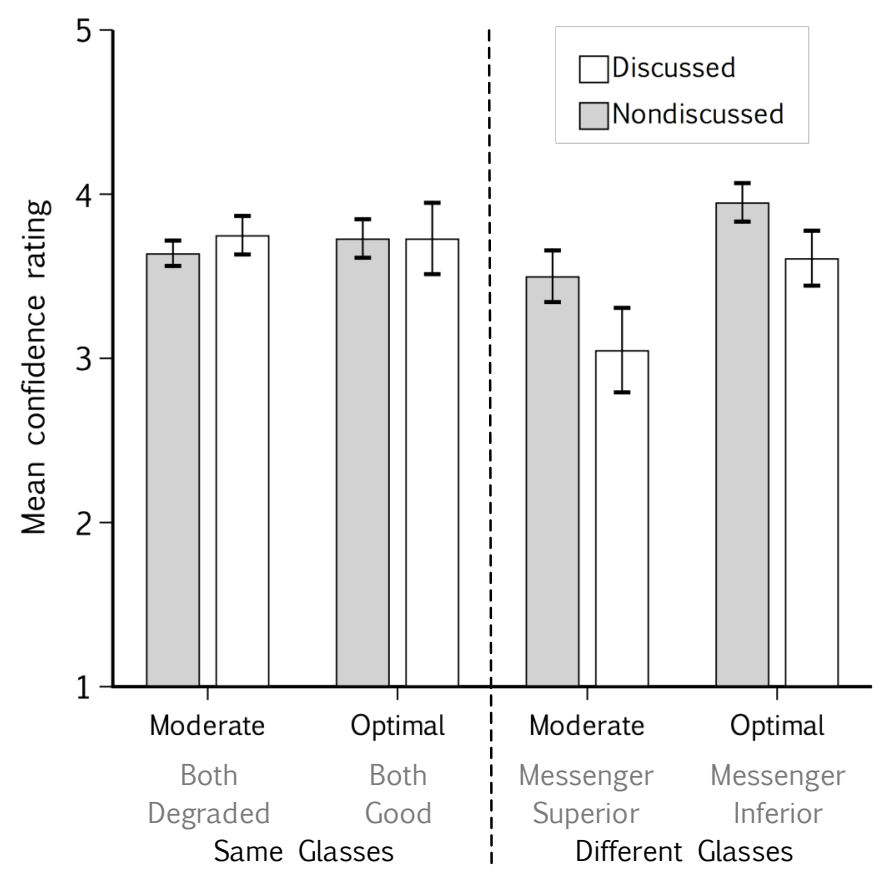

Figure 7.7. Mean confidence for nondiscussed and discussed details by expectancy condition. Error bars depict standard errors of the means.

I next compared subjects' confidence in their correct and incorrect responses; these data appear in Figure 7.8. As the figure illustrates, across conditions, subjects were more confident in their true memories than their false memories, $F(1,68)=40.09, p<.01, f=0.77$. Additionally, there was a trend that subjects who wore different glasses-the messenger-superior and messenger-inferior groupswere less confident in their memories than subjects who wore the same glassesthe both degraded and both-good groups $-F(1,68)=3.20, p=.08, f=0.18$. 


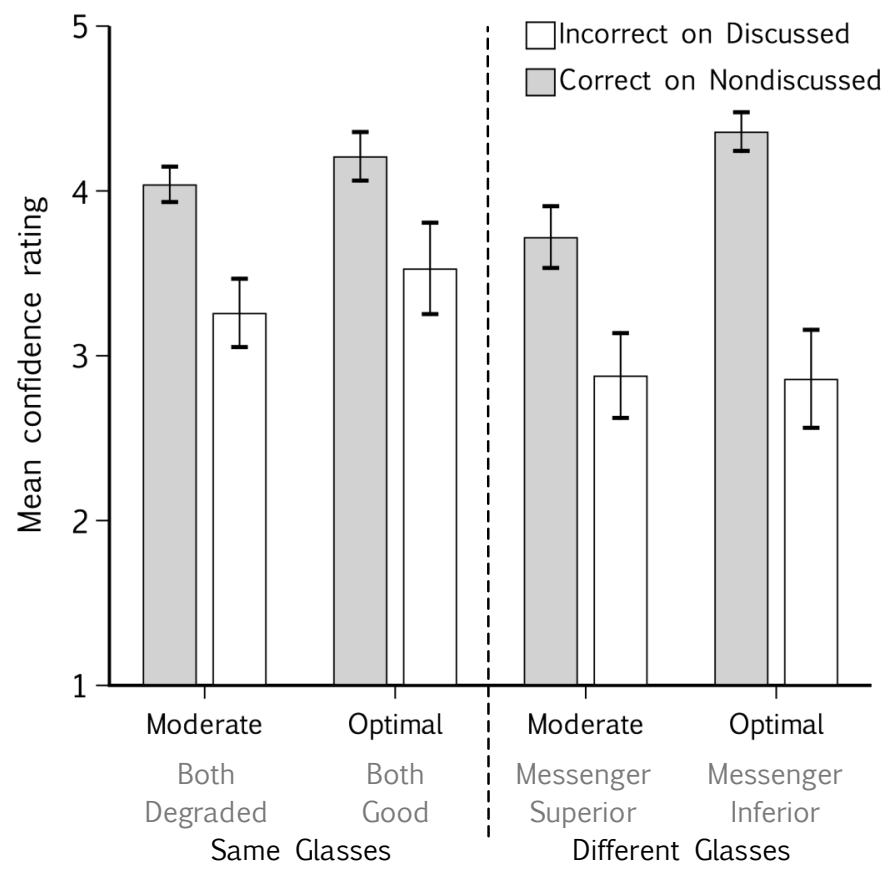

Figure 7.8. Mean confidence for correct responses on nondiscussed details (true memories) and incorrect responses on discussed details (false memories) by expectancy condition. Error bars depict standard errors of the means.

Follow-up t-tests confirmed that this effect was driven by subject-pairs who wore different glasses being less confident when they were misled. Subjects who wore different glasses and subjects who wore the same glasses were just as confident in their correct responses on nondiscussed details, $t(7 \mathrm{O})=1.10, p=.27$, but subjects who wore different glasses were less confident in their incorrect responses on discussed details, $t(70)=1.91, p=.06$, Cohen's $d=0.44$. Taken together, these results suggest that subjects who were told their abilities were different from the misinformation messenger's were not very trusting of what the messenger said, regardless of whether they expected the messenger to be inferior or superior. Additionally, subjects in the messenger-superior group were less confident in their correct memories than subjects in the messenger-inferior group, providing more evidence for the mechanism by which subjects who had superior memories were more misled than their discussion partners with inferior memories, $t(46)=2.67, p=.01$, Cohen's $d=0.77$. 
Gauging subjects' expectations. One limitation to the results reported so far is that I did not consider what subjects actually thought of the misinformation messenger or of themselves. Another way to look at how people's expectations affect their susceptibility to false memories is to examine their ratings on the manipulation checks, and see how those ratings relate to memory performance. I next conducted a correlation analysis between subjects' mean self-partner comparison scores and their proportion correct scores for discussed details. The better that subjects rated themselves compared to the misinformation messenger, the more accurate they were at remembering discussed details, $r=$ $.38, p<.01$. This result provides more evidence that perceptions of credibility might work to both increase and decrease people's susceptibility to false memories: people who thought the messenger was less credible were less misled and people who thought the messenger was more credible were more misled. One limitation to this analysis is that subjects completed the manipulation check at the end of the study; instead of subjects being influenced to the extent they thought their partner was credible, they might have rated their partner as credible to the extent that they were influenced.

\section{Features of the Discussion}

As in the previous experiments, I next examined the features of subjects' discussions. Once again, there were very few instances where one subject disputed the other and the other did not reciprocate (18 out of a possible $253 ; 7 \%$ ); as in the previous experiments, I examined whether the subject disputed what his or her partner said. I grouped subjects according to what they were told about their own and their partner's abilities, and for each of the 253 instances that subjects were exposed to misinformation, I examined the proportion of instances that each discussion feature occurred, and the proportion of times that subjects went on to be misled; these data appear in Table 7.2. 
As Table 7.2 illustrates, the discussion features occurred at similar rates across three of the four groups; however, for the fourth group-the messenger-inferior group - these rates were different. As the first two lines of Table 7.2 show, and consistent with the previous experiments, people were misled more often when they used the misinformation as their answer in the discussion. However, as Table 7.2 illustrates, subjects in the messenger-inferior group used the misinformation as their discussion answer less often than subjects in the other three groups, suggesting that they resisted the messenger's suggestions. Like the previous experiments, subjects occasionally answered with an alternative during the discussion, but changed their answer to the misinformation at test; this behaviour was rare across all four groups.

Table 7.2 also shows that subjects were misled less often when they disputed their partner's suggestions. Interestingly, subjects in the messenger-inferior group disputed their partners more often than subjects in any other group, again supporting the idea that they resisted the misinformation messenger's suggestions. Additionally, when subjects did dispute their partners, subjects in the messenger-superior group were misled more often than subjects in the other three groups, suggesting that even though these subjects initially disagreed they were eventually persuaded by the apparently superior misinformation messenger.

When subjects were exposed to misinformation, they spoke first less often, and, when they did speak first, they were misled less often. However, this pattern was also slightly different for the messenger-inferior group. Although these subjects were misled less often when they spoke first, they spoke first more often than subjects in the other three groups. Additionally, when they did speak second, subjects in the messenger-inferior group were misled less often than subjects in the other groups. 
Table 7.2

Proportion discussion feature occurrence and subsequent proportion of subjects misled for 253 instances that subjects were exposed to misinformation

\begin{tabular}{|c|c|c|c|c|c|c|c|c|}
\hline \multirow{4}{*}{$\begin{array}{l}\text { SUBJECT'S CLASSES } \\
\text { Discussion Features }\end{array}$} & \multicolumn{8}{|c|}{ PARTNER'S GLASSES } \\
\hline & \multicolumn{4}{|c|}{ Same } & \multicolumn{4}{|c|}{ Different } \\
\hline & \multicolumn{2}{|c|}{$\begin{array}{c}\text { Moderate } \\
(\mathrm{n}=61 \text { cases }) \\
\text { Both Degraded }\end{array}$} & \multicolumn{2}{|c|}{$\begin{array}{c}\text { Optimal } \\
(\mathrm{n}=61 \text { cases }) \\
\text { Both Good }\end{array}$} & \multicolumn{2}{|c|}{$\begin{array}{c}\text { Moderate } \\
(n=74 \text { cases }) \\
\text { Messenger Superior }\end{array}$} & \multicolumn{2}{|c|}{$\begin{array}{c}\text { Optimal } \\
(\mathrm{n}=57 \text { cases }) \\
\text { Messenger Inferior }\end{array}$} \\
\hline & $\begin{array}{l}\text { Feature } \\
\text { (n cases) }\end{array}$ & $\begin{array}{c}\text { Misled } \\
\text { (n cases) }\end{array}$ & $\begin{array}{l}\text { Feature } \\
\text { (n cases) }\end{array}$ & $\begin{array}{l}\text { Misled } \\
\text { (n cases) }\end{array}$ & $\begin{array}{c}\text { Feature } \\
\text { (n cases) }\end{array}$ & $\begin{array}{c}\text { Misled } \\
\text { (n cases) }\end{array}$ & $\begin{array}{l}\text { Feature } \\
\text { (n cases) }\end{array}$ & $\begin{array}{c}\text { Misled } \\
\text { (n cases) }\end{array}$ \\
\hline \multicolumn{9}{|l|}{ Answer } \\
\hline Alternative & $.43(26)$ & $.04(1)$ & $.46(28)$ & $.07(2)$ & .39 (29) & $.10(3)$ & $.72(41)$ & $.12(5)$ \\
\hline \multicolumn{9}{|l|}{ Dispute } \\
\hline Dispute & $.39(24)$ & $.08(2)$ & $.41(25)$ & $.04(1)$ & $.47(35)$ & $.23(8)$ & $.67(38)$ & $.16(6)$ \\
\hline No Dispute & $.61(37)$ & $.81(30)$ & $.59(36)$ & $.78(28)$ & .53 (39) & $.79(31)$ & .33 (19) & $.68(13)$ \\
\hline Spoke Second & $.67(41)$ & $.63(26)$ & $.67(41)$ & $.61(25)$ & $.73(54)$ & $.59(32)$ & $.53(30)$ & $.40(12)$ \\
\hline OVERALL PERFORN & & $.52(32)$ & & $.48(29)$ & & $.53(39)$ & & $.33(19)$ \\
\hline
\end{tabular}

Note. Proportion Correct = $1-$ Proportion Misled. For each condition ( $\mathrm{n}$ cases Correct $=$ total $\mathrm{n}$ cases $-\mathrm{n}$ cases Misled). 
Finally, based on proportions, subjects in the messenger-inferior group were misled less often than subjects in the other three groups. Additionally, based on the actual instances that subjects were misled, subjects in the messenger-superior group were misled more often than the other three groups. These subjects were exposed to more misleading details than any other group; this result suggests that these subjects fell prey to their increased opportunity to be misled.

In sum, subjects' expectations about themselves and about the misinformation messenger affected both their behaviour during a social interaction and their susceptibility to misinformation. Subjects who were told they were superior mentioned misinformation to their partner more often, relied on their partner's suggestions less often, and disputed what their partner said more often. Subjects who were told they were equal to the misinformation messenger exhibited very similar patterns of discussion behaviour and memory responses, regardless of whether their expectations were high or low. Subjects who were told they were inferior were misled more often because they were exposed to more misleading suggestions. Taken together, the results provide more evidence that people's expectations about themselves work in combination with their expectations about their discussion partners to determine their susceptibility to false memories.

\section{Predicting False Memories}

I next examined if any discussion features predicted whether subjects would be misled on a given detail. I conducted the same analyses as Experiment 5 . Consistent with Experiment 5, neither subjects' expectations of their own abilities nor their expectations of their partner's abilities predicting whether they were misled in either regression.

All-items regression. When I followed Gabbert et al.'s (2006) approach and included all 384 instances that subjects were asked to discuss a critical detail, 
regardless of whether they were exposed to misinformation, three factors predicted whether subjects were misled. First, the odds ratio showed that subjects who used the misinformation as their discussion answer were 28 times more likely to be misled than those who did not, $\chi^{2}(1, N=384)=96.33, p<.01$. Second, subjects who disputed what their partner said were nearly three times less likely to be misled, $\chi^{2}(1, N=384)=7.51, p<$. .o1. Finally, consistent with Gabbert et al., subjects who spoke first during the discussion of a critical detail were nearly four times less likely to be misled, $\chi^{2}(1, N=384)=15.58, p<.01$.

Exposure-only regression. When I addressed Lindsay's (2007) concerns by including only the 253 instances where subjects were exposed to misinformation, the results were very similar. First, subjects who used the misinformation as their discussion answer were 20 times more likely to be misled than those who did not, $\chi^{2}(1, N=253)=42.33, p<.01$. Second, subjects who disputed their partners suggestions were four times less likely to be misled, $\chi^{2}(1, N=253)=9.59, p<.01$. Finally, there was a trend that subjects who spoke first were only half as likely to be misled as those who did not, $\chi^{2}(1, N=253)=3.95, p=.05$. In other words, these results fit with those of Experiment 4, and suggest that speaking order may play some role in susceptibility to false memories over and above exposure to misinformation. However, speaking first was still a better predictor of being misled when exposure to misinformation was not considered, thus suggesting that it's main influence is in determining whether people will be exposed to misleading suggestions.

Predicting-exposure regression. Finally, I conducted another nominal logistic regression to see if any features of the discussion predicted whether subjects would be exposed to misinformation during the discussion of a given detail. Consistent with the previous experiments, subjects who disputed their partner's 
suggestions were 8 times more likely to be exposed to misinformation, $\chi^{2}(1, N=$ $384)=35.57, p<.01$; subjects who spoke first were 6 times less likely to be exposed to misinformation, $\chi^{2}(1, N=384)=26.39, p<.01$; and there was an interaction between the two factors, subjects who disputed what their partner said were only half as likely to be exposed to misinformation if they spoke first, than if they spoke second, $\chi^{2}(1, N=384)=4.27, p=.04$.

\section{Summary}

In sum, people's expectations about themselves and about other people can affect their susceptibility to misleading suggestions made during a conversation. Specifically, these results build on previous research by showing that it is not only people's perceptions of the misinformation messenger that determine their susceptibility to misleading suggestions, but also their perceptions of themselves (for example Ceci et al., 1987; Dodd \& Bradshaw, 1980; Echterhoff, Hirst, et al., 2005; Vornik et al., 2003). In this study, the influence of ostensibly able and notso-able misinformation messengers depended on people's expectations about their own abilities: when people expected to have similar abilities to the messenger, they were equally influenced by the messenger's suggestions regardless of whether those expected abilities were good or not-so-good. Yet when the messenger was apparently superior, people were more influenced, and when the messenger was apparently inferior, people were not influenced. Even the most credible and knowledgeable misinformation messenger may not influence people's memories if those people perceive their own abilities to be better.

Consistent with earlier research, people who were told they were at a disadvantage were more misled than people who were told they were at an advantage (Gabbert et al., 2007; Skagerberg \& Wright, 2008). Yet my experiment also builds on the previous findings by showing that this difference is caused in 
part by the apparently advantaged people being protected from false memories, not simply by the apparently disadvantaged people being more susceptible to false memories.

Additionally, the results show that people's expectations about themselves and about other people can affect their behaviour during a conversation. People who were told they were superior to their discussion partner behaved quite differently to people who were told they were inferior and people who were told they were the same. For instance, subjects who were told they were better conveyed more misleading suggestions than any other group. Perhaps in real life, people who see themselves as more credible and knowledgeable than others might pass on more information than vice versa. As a result, the people they talk to might be more likely to experience false memories simply because they have more opportunity for their memory to be distorted. Of course, to the extent that the new information passed on is accurate, this mechanism may be beneficial, and may help people who know less to know and remember more. Yet, these results suggest that this helpful mechanism might become unhelpful when the information conveyed is bad. One additional problem is that people who believe they are very knowledgeable and credible may not necessarily be either knowledgeable or credible (Dunning, Johnson, Ehrlinger \& Kruger, 2003; Kruger \& Dunning, 1999).

In fact, in this study people who expected to have degraded vision actually remembered critical details better than their counterparts who expected to have optimal vision. Yet, despite having better memories, these people were less confident in their memories; the people with worse memories were more confident. In short, people's perceptions about their own abilities, and their perceptions about other's abilities can be misguided. Unfortunately these perceptions can affect not only people's baseline memory accuracy but also their 
susceptibility to misleading suggestions. Future research might investigate how to create the ideal balance between encouraging people to perform at their optimum, while also encouraging them to be confident in their performance; such research might be useful for educational settings and work places.

These results also build on cognitive-enhancing and memory-dampening placebo research by showing that it is not necessary for people to ingest an inert substance to change their expectations about their memory ability and their susceptibility to false memories (Assefi \& Garry, 2003; Clifasefi et al., 2007; Parker et al., 2008). Simply changing the dynamics of a social interaction and telling people that they had the same or different abilities as their discussion partner led people to be more or less influenced by their partner's misleading suggestions.

Finally, other research shows that people are more influenced by misinformation conveyed in a social interaction than misinformation conveyed in a non-social way, such as through a written narrative (Gabbert et al., 2004; Paterson \& Kemp, 2006b). The results of this study suggest that the influence of misinformation conveyed in a social interaction might depend on factors in that social interaction. In some situations—such as those in Gabbert et al.'s (2004) and Paterson and Kemp's (2006b) studies—socially conveyed information may be more persuasive, but in other situations-such as when people are very sure of their own abilities or very confident in their own memories-it may not be persuasive at all. 


\section{Chapter 8}

\section{General Discussion}

There were two overarching questions in this thesis. First, would people influence each other's memories of a shared experience when they discussed it? Second, would different social dynamics in the discussion affect how much they influenced each other's memories?

To address these questions I used the MORI technique to show pairs of people slightly different movies on the same screen at the same time. As a result, pairs of people shared an experience but each had systematic differences in their memories of the experience, and the effect of those differences could be tracked through discussion.

\section{Overview of Key Results}

Across nine experiments, there were three major findings. First, I found that the MORI technique was an effective means to show people different movies without them realising they each saw something different. When people watched a movie via the MORI technique, they could see and remember the details of the movie (Experiments $1 \mathrm{~A}$ and $1 \mathrm{~B}$ ) and they did not notice or implicitly remember details from the alternate (blocked) movie version (Experiments $3 \mathrm{~A}$ and ${ }_{3} \mathrm{~B}$ ).

Second, I found that people who shared an experience influenced each other's memories of that experience. In other words, people falsely remembered details from their partner's version of the event, even though those details contradicted what they personally saw. Additionally, I generalised this pattern of results across two different events and stimulus sets (Experiments $2 \mathrm{~A},{ }_{2} \mathrm{~B}, 4,5$ and 6).

Finally — consistent with the SMF — the specific social dynamics of people's discussions led them to be both more and less influenced by each other's suggestions (Johnson et al., 1993; Lindsay, 2008). Put another way, the corrupting influence of the discussion depended on social factors in the interaction. 
Specifically, people were more likely to remember false details that their romantic partner suggested than false details that a stranger suggested (Experiment 4), and telling people that their counterpart's vision was better or worse than their own led them to be more or less influenced by their counterpart's false suggestions (Experiment 6).

\section{Source Monitoring Framework}

When people make source monitoring decisions they consider their social evaluations about the person who conveyed the information (Johnson et al., 1993; Lindsay, 2008). Across my five discussion experiments, subjects were influenced by their partner's misleading suggestions, a finding that suggests people generally evaluate others with whom they have shared experiences as credible sources of information about those experiences.

However, although subjects were generally misled by their partner's suggestions, the social dynamics of the conversation played a role. Subjects' relationship with the misinformation messenger, and their expectations of their own abilities and the messenger's abilities, led them to be both more and less influenced by the messenger's suggestions. In short, people do not always see others with whom they have shared experiences as a valuable source of information about those experiences; sometimes these people might be seen as valuable sources, yet other times they might be seen as worthless. People were the most misled by people they knew and trusted, yet completely resisted the suggestions when they afforded the messenger little credit. In real life, these patterns might be adaptive: known and trusted others probably convey valuable information, whereas people with inferior abilities probably do not.

Finally, across the five discussion experiments, $64 \%$ of subjects who were exposed to two or more misleading details were influenced by some of their partner's suggestions but were not influenced by others (218 of 339 people). This 
result fits with the idea that rather than making a blanket assessment of the misinformation messenger and all the information conveyed, subjects probably made their source monitoring decisions based on different dimensions, and assigned different weights to these dimensions in each decision (Johnson et al., 1988; Lindsay, 2008; Mitchell \& Johnson, 2000).

\section{When does the Influence Occur?}

Although subjects' responses on the independent test can be explained as source monitoring errors, it is not clear when the influence or the errors actually occurred. Perhaps subjects were influenced during the discussion, and their responses at test simply reflected the earlier errors. Alternatively, subjects might have absorbed information throughout the experiment, and made errors only as they tried to answer the relevant discussion questions. In fact, there are a number of different pathways that might have led subjects to respond with the misinformation on the test.

For instance, perhaps during the discussion, while trying to figure out where Chad put the wallet, Mollie remembered seeing Chad stand beside the bench. Because Mollie's memories were activated when her partner (Jack) suggested that Chad stole the wallet, Mollie might have incorporated the new information into her memory, thus affecting her later memory responses (Meade \& Roediger, 2002; Mitchell \& Johnson, 2000). Another possibility is that details from the discussion might work their way into Mollie's memory because she processed them at a "deeper level" than the movie details (Craik \& Lockhart, 1972; Craik \& Tulving, 1975). During the movie Mollie would have to pay attention to a large number of details, but during the discussion she might spend more time and effort thinking about a few specific details, thus she might encode those detailsthe misleading suggestions-more deeply. As a result those details might be more accessible and feel qualitatively different when she completes the final 
memory test, leading her to report the misinformation instead of what she saw (Kronlund \& Whittlesea, 2005). Alternatively, at the test, Mollie might have remembered some details consistent with Chad stealing a wallet, and some details consistent with Chad looking at a wallet but putting it back. When she tried to figure out what Chad actually did in the movie, she might have relied on features of her memories as well as her beliefs and knowledge to do source monitoring and decide which details came from the movie. If Mollie did make an error on the test, it could be because she falsely remembered a misleading detail, or because she falsely believed the detail was correct.

If Mollie did falsely remember a detail from Jack's version of the movie she might have visualised that detail and then later confused the image with reality, incorrectly deciding that she had seen something she only thought and heard about. Alternatively, Mollie might have simply confused what she heard Jack say with what she saw. Another possibility is that Mollie might have capitulated to Jack's suggestion, perhaps because she made a strategic decision that Jack's memory was correct. These types of errors might occur during the discussion or during the test. One final possibility is that Mollie might have capitulated to Jack's suggestion during the discussion—not because she thought he was accurate, but because she felt pressured to do so-and then later she confused what she agreed to in conversation with what she saw. In sum, there are a number of ways that subjects might come to answer with the misinformation at test; we do not know whether the influence occurs during the discussion or during the test. My results provide some evidence for both possibilities.

Discussion. Across the five discussion experiments, the majority of the time that subjects used the misinformation as their discussion answer they subsequently used it as an answer on the independent test (439 of 544 instances; weighted mean $=81 \%$ ). In fact, when subjects answered with the misinformation 
during the discussion they were up to 87 times more likely to be misled on the final test. These results suggest that subjects were influenced by the suggestions during the discussion, and that influence persisted until the final test.

Test. But, subjects' discussion answers did not always match their independent test responses. Some subjects did not answer with the misinformation during discussion, but went on to use it as their answer at test, suggesting that they were influenced after-rather than during — the discussion. Additionally, some of the time that subjects answered with the misinformation during discussion, they subsequently reverted to the correct answer at test (105 of 544 instances; weighted mean $=19 \%$ ) suggesting that they only agreed with their partner in the discussion to be polite or to avoid arguing. In short, misinformation did not work its way into subjects' independent reports simply because they used it as a discussion answer, and it still worked its way into their independent reports even when they did not use it as a discussion answer. Perhaps the influence occurred during both the discussion and the test.

Of course, I do not know whether subjects actually remembered the false details they reported, or whether they simply reported those details because they believed them to be accurate. Although I told subjects that I was testing their memory for the movie, some may have reported their partner's suggestions even though they had no memory of those details.

Future research might attempt to distinguish whether people actually remember or simply believe that the misinformation is correct. For instance, future research might use a source monitoring test to examine whether subjects remember seeing the false details that their partner suggested. To isolate where the influence occurs, future research could combine the basic elements of my studies with Lindsay's (1990) logic of opposition study. Specifically, subject-pairs could be warned just before the test that they watched different movies, and that 
any information their partner suggested was wrong. If subjects still reported misinformation on the test it would show that they remembered that information, and that their memories were influenced before the test.

Considered as a whole, these data provide evidence that people can be influenced by misleading suggestions conveyed in a conversation about a shared experience, and that the specific social dynamics of the conversation can lead people to be either more or less influenced by those suggestions. However, questions about when people are influenced and why they are influenced remain.

\section{Remembering in Groups}

Some of the broader literature on group behaviour provides insight as to why subjects might be influenced by their partner's suggestions.

\section{Informational and Normative Influences}

As described in Chapter 1, social pressure can lead people to provide responses that contradict their perceptions (Asch, 1952; 1953; 1966). When people provide false responses, sometimes they believe that the responses are correct (informational influence) and other times they simply capitulate because of the social pressure of the situation (normative influence; Deutsch \& Gerard, 1955).

Subjects in my studies appeared to demonstrate both informational and normative influence. Recall that $81 \%$ of the time that people used the misinformation as their discussion answer, they subsequently used that answer on the independent test, a finding suggesting that they capitulated to their partner's suggestion because they believed the information was correct. These people might have strategically decided that their partner's answer was accurate, or they might have come to falsely remember seeing the details their partner suggested. The remaining $19 \%$ of the time that subjects used the misinformation as their discussion answer, they reverted to the correct answer from their own 
version on the test, a finding suggesting that they capitulated to their partner's suggestion only because of the social pressure of the situation. In short, the social context of the discussion might have led subjects to believe their partner's responses were accurate, or might have pressured them not to challenge their partner's suggestions.

One caveat is that I did not set out to measure these two influences, so my results provide only a rough measure. Specifically, in my experiments subjects always provided a public answer during the discussion, so it is impossible to know whether their discussion responses were driven by the pressure to capitulate, or by a genuine belief that their partner's suggestions were correct. When subjects agreed with their partner during the discussion and subsequently conformed on test, I deemed their responses to demonstrate informational influence. Perhaps in some of these instances, subjects initially agreed due to social pressure, and then made a source monitoring error at test-confusing what they agreed to with what they believed to be the answer. In other words, some instances that I deemed to be informational influence might have been driven by normative influence. Likewise, when subjects agreed with their partner during the discussion but reverted to their own answer on the test, I deemed their responses to demonstrate normative influence. Yet some subjects may have actually believed their partner during the discussion, and later made a source monitoring error in the other direction, answering with the correct information on the test instead of what their partner told them. Future research could examine the role of these influences in subjects' discussion responses by comparing discussion responses made privately and publicly. Additionally, future research might examine how each influence contributes to false memories by comparing independent test responses that follow private and public discussion responses. 


\section{Collaborative Inhibition}

Instead of being influenced by social pressure, another possibility is that the experience of remembering with another person affected what subjects remembered and reported. People who remember in groups remember less than they would if each member remembered individually and the members' memories were pooled (for example see Andersson \& Ronnberg, 1997; Meudell, Hitch \& Kirby, 1992; Takahashi \& Saito, 2004; Weldon, 2001; Weldon \& Bellinger, 1997). This finding is known as collaborative inhibition, and has a variety of social and cognitive explanations (Weldon \& Bellinger, 1997).

One explanation is social loafing (Latané, Williams, \& Harkins, 1979); people may remember less in a group setting because they can rely on others to do the work; as a result they might not put in much effort to search their own memories (Weldon \& Bellinger, 1997; Weldon, Blair \& Huebsch, 200o). Alternatively, perhaps people do not convey everything they remember because they are worried about looking bad-this explanation overlaps with normative influence (Deutsch \& Gerard, 1955; Stasser, 1999). Another possibility is that the social interaction itself makes it more difficult for people to retrieve relevant information; this kind of disruption could occur in at least two ways. First, it might be that each person's ability to remember is actually disrupted by being exposed to what other people remember. Specifically, when people hear someone else suggest an answer, it might disrupt their own retrieval strategies, making it difficult for them to recall what they actually saw (for example see Finlay, Hitch, \& Meudell, 20oo; Basden, Basden, Brynor, \& Thomas, 1997; Wright \& Klumpp, 2004). A second possibility is that the act of communicating might work to deplete people's cognitive resources (Gilbert, Pelham, \& Krull, 1988). As a result, the communicators will have fewer resources available to discern whether suggested information is correct or to find an alternative answer, 
perhaps leading them to agree with their partner's suggestions.

Whether socially or cognitively driven, collaborative inhibition might explain why subjects in my experiments did not always expose each other to the misinformation, and why they were influenced by each other's suggestions. Across the five discussion studies, when critical details acted as nondiscussed details, subjects remembered the correct detail from their version of the event $78 \%$ of the time (on the independent test; 1241 of 1600 instances). However, when critical details acted as discussed details, subjects mentioned the correct detail from their version of the event-the misinformation-only $64 \%$ of the time (during the discussion; 1030 of 1600 instances). Taken together, these results suggest that subjects could remember the critical information, but did not always convey it in the discussion. Collaborative inhibition might lead subjects to be less likely to mention misinformation, and thus more likely to answer with their partner's misleading suggestions—at least during the discussion.

However, in my experiments, people were not influenced as soon as a false detail was suggested; instead, people often disputed their partner's suggestions. Across the five experiments, half of the time that people were exposed to misinformation they disputed it (517 out of 1030 instances). Additionally, $17 \%$ of the time, subjects who disputed their partner's suggestions subsequently answered with the misinformation on the final test, showing that they were influenced, but not as a result of inhibition during the discussion. In sum, collaborative inhibition cannot fully explain why people were influenced by their partner's suggestions. Expertise and Group Remembering

One factor that might play a role in determining whether subjects will mention misinformation to their discussion partner-and whether they will dispute their partner's misleading suggestions-is their perceptions of expertise. 
When remembering in groups, people generally talk about information that other members already know, rather than new or unique information (Larson, Foster-Fishman, \& Franz, 1998; Larson, Foster-Fishman, \& Keys, 1994; Stasser, Stewart, \& Wittenbaum, 1995; Stewart \& Stasser, 1995; Wittenbaum, 1998). This behaviour is especially true of members who have little experience on the task at hand; people who have previous experience on a given task are just as likely to mention unique and shared information (Wittenbaum, 1998). Additionally, when expert roles are assigned or are made known to group members, or when group members already know each other-and each other's areas of expertise-more unique information is mentioned during discussion (Stewart \& Stasser, 1995; Stasser, Stewart, \& Wittenbaum, 1995).

Consistent with the social explanations of collaborative inhibition and normative influence, these findings suggest that group members might be worried about looking bad if they mention information that is new to other group members, especially if they are worried about being wrong or challenging other members' memories, and especially if they see other people as more expert than themselves (Stasser, 1999).

In my studies, each member of a subject-pair had unique memories of the critical details. If Jack mentioned a misleading detail during the discussion, Mollie would have to challenge Jack's suggestion to mention the uniquemisleading — detail from her version. If Mollie judged herself to be non-expert or was reluctant to open herself up to criticism or rejection she might have decided not to mention the opposing detail. In fact, the results of Experiment 6 suggest that subjects' perceptions of expertise did play some role in exposure to misleading suggestions. Subjects in the messenger-inferior group-those who saw themselves as the expert in the interaction-conveyed more unique information (misleading suggestions) and disputed their partner's suggestions 
more often than any other group. These results provide more evidence that social pressure might have led subjects not to mention the misinformation, and to use their partner's suggestion as their discussion answer. Additionally, the results also suggest that the social pressure of a situation might depend on people's perceptions of expertise within that situation.

Transactive Memory Systems

Subjects' judgments about expertise affected not only how much misinformation they conveyed and how much misinformation they were exposed to, but also how influenced they were by misinformation. These results fit with the literature on Transactive Memory Systems (Wegner, 1987; Wegner et al., 1985). Specifically, consistent with transactive memory theory, my results demonstrated that subjects relied on other people's memories to supplement their own memories, that subject-pairs who were in romantic relationshipspresumably with established transactive memory systems-were more likely to rely on each other's memories than subject-pairs who were not in relationships, and that subjects' perceptions of each other's abilities affected how much they relied on each other's memories.

Transactive memory literature suggests that people rely on each other within different domains of expertise; instead of one person always relying on the other, each person benefits from the other's expertise (Hollingshead, 1998b; Hollingshead, 20oo; Stephenson et al., 1991; Wegner, 1987; Wegner et al., 1985). Perhaps subject-pairs in romantic relationships were more misled than other subjects because they knew who would be more expert at remembering each critical detail, and were comfortable relying on the expert's suggestion. Although I examined only global expertise judgments-where each person was seen as better or worse in general - there was some evidence that people did make judgments about expertise in different domains to decide whose memories 
were the most reliable (for examples, see Chapter 5). Future research might examine whether judgments about domain-specific expertise affect false memories. People with expertise in different areas—or people who believe they have expertise in different areas-might be more or less susceptible to influence in those and other areas.

Finally, my findings build on the previous research by suggesting that transactive memory systems may not always be beneficial; although relying on each other's memories gives people an opportunity to increase they amount they remember, when someone conveys false information, they may come to remember that false information too.

\section{Implications}

Although my research does not tell us the precise mechanisms driving false memories, it has important theoretical, methodological and practical implications.

\section{Source Monitoring Framework}

First, these findings help us to understand the role of social features in the SMF. Although it is widely accepted in the SMF that people's appraisals about the messenger affect their source monitoring decisions (for example see Cook et al., 2003; Hicks \& Cockman, 2003; Holtgraves et al., 1989; Johnson et al. 1993; Lindsay, 2008; Mather et al., 1999), very little research has investigated how these social evaluations might operate in actual social interactions. To examine how people's evaluations of their conversational partners affect their source monitoring decisions, I first established that people's memories were influenced by new information conveyed in a conversation about a shared experience. Next, to see what role different social judgments played in these source errors, I varied the nature of the pairs-comparing people who knew and trusted each other with those that did not—and I varied people's perceptions of their own and each 
other's abilities. Both of these factors affected people's susceptibility to false memories. Of particular interest was the finding that people's perceptions of the source were only important in relation to their perceptions of themselves. That is, people were no more or less influenced by a supposedly good or a supposedly bad source when they were told they had the same abilities as the source; yet, when people were told their abilities were better than the source they were less influenced, and when they were told they were worse then the source they were more influenced. This result suggests that to fully understand how social appraisals affect source monitoring, social appraisals cannot be examined in isolation. For instance, telling people information is provided by a credible or non-credible source neglects the important aspect of how those people perceive themselves in relation to the source.

Interestingly the same factors that influenced source monitoring (subjects' test responses) also influenced social capitulation (subjects' discussion responses). For instance, people in romantic relationships appeared not to demonstrate normative influence at all-they never went along with their partner only to be polite in the discussion, or if they did, they maintained their prior commitment in the independent test. Additionally, people who were told they were superior to their partners capitulated to their partners' suggestions less often-and disputed their partners more often-than subjects in the other groups. Finally, subjects who were told they were inferior to their partners capitulated to their partner's suggestions most often. In short, subjects' social appraisals led them to be both more and less likely to capitulate during the discussion. Put another way, some of the factors used in the SMF to explain retrospective source monitoring decisions also explain immediate decision-making behaviour. For instance, subjects in Asch's $(1952 ; 1953 ; 1966)$ studies might have capitulated to the confederate's responses because they judged the confederates to be a credible 
source of information. In fact, Deutsch and Gerard's (1955) research supports this explanation—subjects sometimes capitulated even though they gave private responses, suggesting that they took the confederates' claims to be more credible than their own perceptions.

In sum, people's relationships with each other, and their perceptions of their own and each other's abilities led them to be more and less likely to capitulate during the conversation, and to be more and less susceptible to source monitoring errors following the interaction. These results suggest that people make the social appraisals that affect their source monitoring decisions during their social interactions. An interesting question that remains is whether these appraisals might also affect people's source decisions in retrospect. For instance, if someone discovered only after a conversation that her partner had an ulterior motive, would she be able to resist the influence of that person's suggestions?

One study suggests that people might be able to use these kinds of judgments in retrospect; Echterhoff, Hirst, et al. (2005) showed that people resisted the influence of misinformation when they were warned that the messenger was not reliable, even though the misinformation had already been presented. Yet, in that study, misinformation was presented in a written form, and may have missed the vast array of social dynamics that would be at play during an actual interaction. Perhaps people would not be able to resist the influence of misinformation conveyed in a conversation, especially given that the social dynamics of the conversation might have already led them to capitulate to their partner's suggestions. A future study might attempt to address this question by adapting the design of Experiment 6 in line with Echterhoff, Hirst, et al. Specifically, pairs could be told what type of acuity glasses each member wore only after the discussion. If subjects are more and less misled based on the postdiscussion warning, it would suggest that people can consider social appraisals 
retrospectively when they make source decisions; if subjects' responses are not affected by the post-discussion warning, it would suggest that social appraisals are important only during their actual interactions.

Considered as a whole, this research builds on other source monitoring research by highlighting the importance of the social context for examining social aspects of source monitoring. Of course, I examined only two of potentially hundreds of social factors that might influence people's source monitoring decisions; the MORI technique provides unique means to examine other social factors.

\section{The MORI Technique}

In my experiments, pairs of people sat next to each other and watched ostensibly the same event on the same screen at the same time, while systematic differences were brought into their memories of the experience; as a result, the effect of those differences could be tracked through a real-life discussion about a genuinely shared experience.

The results show that the MORI technique provides an effective way to investigate the role of a variety of social factors in false memories. For instance, future research might examine false memories in different work and family relationships. Additionally, future research might follow up Asch's (1952; 1953; 1966) idea that different personality dispositions lead people to be more or less influenced in social interactions. Perhaps researchers could pair-for example-

more extraverted and more introverted people to see whether these personality dispositions also affect susceptibility to false memories.

\section{Measuring False Memories}

One difference between my experiments and other memory conformity research is the way I operationalised "discussed" details. I was interested in how people's memories would be affected when someone who had shared an 
experience conveyed misinformation. To address this question, I counted "discussed" details as details where subjects were exposed to misinformation. However, other researchers examine details mentioned during pairs' discussions without differentiating between subjects who were and were not exposed to misinformation (see for example Gabbert et al., 2006; Gabbert et al., 2007).

To be concrete, consider the example of Jack-who saw Chad steal the wallet—and Mollie—who saw Chad put the wallet back. Let's say Jack told Mollie that Chad stole the wallet, and Mollie did not respond. In short, Mollie and Jack talked about the wallet detail; Mollie was exposed to misinformation, but Jack was not. In my research, this detail would be counted as a discussed detail for Mollie but not for Jack. By contrast, in Gabbert et al.'s (2006; 2007) research it would be counted as a discussed detail for both Mollie and Jack. These different approaches have generated inconsistent findings.

For instance Gabbert et al. (2006; 2007) found that speaking order predicts whether people will be misled: people who speak first are less likely to be misled than people who do not speak first. Consistent with these studies, across my experiments, when I examined all instances that subjects discussed a critical detail, regardless of whether they were exposed to misinformation, I found that people who spoke first were less likely to be misled. However, as Lindsay (2007) argued (see Chapter 3), people who spoke first might have been exposed to less misinformation, thus decreasing the risk that they would be misled.

When I repeated the same analyses, examining only the instances that people were exposed to misleading details, speaking order was not a good predictor of being misled: in three experiments speaking order did not predict being misled at all, and in two experiments speaking order was only a marginal predictor of being misled. Additionally, across all five experiments, speaking order predicted exposure to misinformation. In short, consistent with Lindsay's (2007) concerns, 
people who spoke first were less likely to be exposed to misinformation, thus protecting these people from being influenced by that misinformation.

Taken together, these results show that people who speak first are less likely to be misled. Yet speaking order determines exposure to misinformation, and not necessarily susceptibility to false memories. Theoretically it is important for researchers to decide how to address exposure to misinformation, and to draw conclusions based on their approach. In short, whether subjects' responses should be included in the analyses when they have not been exposed to any misinformation depends on the research question.

\section{Costs and Benefits of Memory Distortion}

In my experiments, I investigated only the negative effects of discussion on memory by creating a no-win situation: information correctly reported by one member of a pair was incorrect if adopted by the other member. But the mechanisms driving the effect I found might also work to improve memory accuracy outside of this specific experimental situation. Because people do not remember everything they see, they might increase their ability to report accurate information about an event in the same way they incorporate inaccurate information (Meade \& Roediger, 2002). In other words, people might come to know and remember more than they otherwise would by absorbing information from lots of different sources. Presuming that most of the information conveyed in the world is good information, the effect of the mechanism should generally be good. However, when the information conveyed is bad, the mechanism might end up producing negative effects, like those found in my experiments. In short, whether discussion leads to positive or negative outcomes for memory depends in part on the accuracy of the information conveyed during discussion. In situations where the content of people's memories is important—such as in the justice system—the consequences of 
discussion might be serious.

Eyewitness Memory

The justice system relies on people to accurately remember what happened at a crime or accident. Additionally, corroboration is taken as evidence of accuracy; when two people recall the same details their testimony is given more credit than the testimony of either person alone (Pickel, 1993). Yet, my research suggests that people may report the same details as the result of post-event discussion, rather than because they each noticed and remembered those details. If one witness makes an error, other witnesses might also come to remember incorrect details, increasing the consistency of their testimony, but decreasing their accuracy. Witnesses' consistency with each other's reports may convince them of their own accuracy, increasing their confidence in their memories (Luus \& Wells, 1994). Additionally, confident witnesses seem more credible to other people (Bradfield \& Wells, 2000; Brewer \& Burke, 2002). As a result, investigators and jurors may give the consistent and confident reports more credit than they are due when weighing up the evidence in a case, potentially misleading the course of the investigation and the outcome of the trial. In fact, the same kinds of problems might occur in any situation where the transmission of information is important.

\section{Transmission of Information}

People in work places, educational settings, social or family groups might incorporate one person's error into their own memories, and as a result be led to the wrong course of action, disrupting their goals or productivity. To the extent that the information conveyed in social interactions is accurate, groups will probably benefit from the interaction. But if one person gets some information wrong, it could lead others to remember false information. However, my research suggests that whether people will be influenced depends in part on who 
conveys the false information, and how they judge that person. To the extent that people's perceptions of their own and other's expertise are accurate, these processes might work to their benefit. Although people might experience benefits as a result of remembering more accurate information, it is dubious to conclude that their memories will be "better."

Remembering More Accurate Information.

At face value, remembering more good information seems advantageous. But consider the example of several eyewitnesses who talk about a witnessed event and incorporate accurate details from each other's reports into their own memories. In this case, the eyewitnesses' reports would become more accurate, more informative, and just like in the example above more consistent, more confidently told, more credible-by all accounts the report would be better. Still, it remains a matter for debate whether these memory reports should be considered better-and given the extra credit of corroborated evidence-simply because the new information happened to be accurate.

\section{Mind Over Memory Distortion}

Finally, my results suggest that people exercise some control over the fate of their memories. People's relationships with the misinformation messenger, and their judgments about the messenger and about themselves led them to be more and less likely to incorporate new information into their memories. Future research might investigate potential interventions to protect people from false memories, especially in situations where the content of their memories is important. For instance, in work settings, employers could gauge their employees skills regularly and ensure that team-members are aware of their own and other's skills, thus increasing the chance that people will access good information from true experts, and decreasing the risk that they will be misled by someone less expert and more likely to make errors. Interventions might also 
be valuable in the justice system; it is unlikely that we will ever stop witnesses from discussing their experiences, however we might be able to intervene to protect them from false memories.

\section{Conclusion}

In conclusion, when people share an experience then talk about it, they can come to remember seeing things that they were only told about after the event. Discussion probably works to help us remember more information than we otherwise would-my results suggest that sometimes this memory mechanism might work to sweep up inaccurate details. In situations where the content of people's memories is important, or where their memories are used to guide future behaviour, discussion might create problems. Yet under the right circumstances, people can be protected from false memories that arise from discussion. 


\section{References}

Aberson, C. (2002). Interpreting null results: Improving presentation and conclusions with confidence intervals. Journal of Articles in Support of the Null Hypothesis, 1, $36-4^{2}$.

Alper, A., Buckhout, R., Chern, S., Harwood, R., \& Slomovitz, M. (1976). Eyewitness identification: Accuracy of individual vs. composite recollections of a crime. Bulletin of the Psychonomic Society, 8, 147 - 149.

Andersson, J., \& Ronnberg, J. (1997). Cued memory collaboration: Effects of friendship and type of retrieval cue. European Journal of Cognitive Psychology, 9, $272-287$.

Asch, S. E. (1952). Social Psychology (pp. 450 - 501). NY: Prentice Hall.

Asch, S. E. (1953). Effects of group pressure upon the modification and distortion of judgments. In H. Guetzkow (Ed.), Groups, leadership and men:

Research in human relations (pp. 177 - 190). NY: Russell and Russell Inc.

Asch, S. E. (1966). Interpersonal Influence: Effects of group pressure upon the modification and distortion of judgments. In E. E. Maccoby, T. M.

Newcomb \& E. L. Hartley (Eds.). Readings in Social Psychology ( ${ }^{\text {rd }}$ ed., pp. 174 - 183). London: Methuen \& Co Ltd.

Assefi, S. L., \& Garry, M. (2003). Absolut ${ }^{\circledR}$ memory distortions: Alcohol placebos influence the misinformation effect. Psychological Science, 14, 77 - 80.

Barclay, C. R. (1996). Autobiographical remembering: Narrative constraints on objectified selves. In D. C. Rubin (Ed.). Reconstructing our past: An overview of autobiographical memory (pp. 94 - 125). Cambridge, MA: Cambridge University Press.

Barclay, C. R., \& DeCooke, P. A. (1988). Ordinary everyday memories: Some of the things of which selves are made. In U. Neisser \& E. Winograd (Eds.). Remembering reconsidered: Ecological and traditional approaches to the study of memory (pp. 91 - 125). Cambridge, MA: Cambridge University Press. 
Bartlett, F. C. (1932). Remembering: A study in experimental and social psychology (pp. 239 - 314). Cambridge, MA: Cambridge University Press.

Basden, B. H., Basden, D. R., Bryner, S., \& Thomas, R. L., III. (1997). A comparison of group and individual remembering: Does collaboration disrupt retrieval strategies? Journal of Experimental Psychology: Learning, Memory and Cognition, 23, 1176 - 1189.

Basden, B. H., Reysen, M. B., \& Basden, D. R. (2002). Transmitting false memories in social groups. American Journal of Psychology, 115, 211 - 231.

Belli, R. F. (1989). Influences of misleading postevent information:

Misinformation interference and acceptance. Journal of Experimental Psychology: General, 118, $72-85$.

Betz, A. L., Skowronski, J. J., \& Ostrom, T. M. (1996). Shared realities: Social influence and stimulus memory. Social Cognition, 14, 113 - 140.

Bond, R., \& Smith, P. B. (1996). Culture and conformity: A meta-analysis of studies using Asch's (1952b, 1956) line judgment task. Psychological Bulletin, 119, 111 - 137 .

Bradfield, A. L., \& Wells, G. L. (2000). The perceived validity of eyewitness identification testimony: A test of the five Biggers criteria. Law and Human Behavior, 24, 581 - 594 .

Brewer, N., \& Burke, A. (2002). Effects of testimonial inconsistencies and eyewitness confidence on mock-juror judgments. Law and Human Behavior, 26, $353-364$.

Castle, S. (2003, September 13). Euro vote too close to call with Sweden in mourning. The Independent. Retrieved April 3, 2008 from http://www.independent.co.uk/news/europe/euro-vote-too-close-to-call-withsweden-in-mourning-579787.html

Ceci, S. J., Ross, D. F., \& Toglia, M. P. (1987). Suggestibility of children's memory: Psycholegal implications. Journal of Experimental Psychology: General, $116,38-49$. 
Clifasefi, S. L., Garry, M., Harper, D. N., Sharman, S. J., \& Sutherland, R. (2007). Psychotropic placebos create resistance to the misinformation effect. Psychonomic Bulletin and Review, 14, $112-117$.

Cook, G. I., Marsh, R. L., \& Hicks, J. L. (2003). Halo and devil effects demonstrate valenced-based influences on source-monitoring decisions. Consciousness and Cognition, 12, $257-278$.

Cowell, A. (2003a, September 11). Swedish foreign minister wounded in knife attack while shopping. New York Times. Retrieved April 3, 2008 from http://query.nytimes.com/gst/fullpage.html?res=9Eo3E6DC133BF932A2575A CoA9659C8B63

Cowell, A. (2003b, September 12). Sweden's foreign minister dies of wounds from attack. New York Times. Retrieved April 3, 2008 from http://query.nytimes.com/gst/fullpage.html?res=9Bo3E1DA103BF931A2575A CoA9659C8B63\&sec=\&spon $=\&$ pagewanted $=$ all

Cuc, A., Ozuru, Y., Manier, D., \& Hirst, W. (2006). On the formation of collective memories: The role of a dominant narrator. Memory and Cognition, 34, $752-762$.

Craik, F. I. M. \& Lockhart, R. S. (1972). Levels of processing: A framework for memory research. Journal of Verbal Learning and Verbal Behavior, 11, 671 -684 .

Craik, F. I. M. \& Tulving, E. (1975). Depth of processing and the retention of words in episodic memory. Journal of Experimental Psychology: General, 104, $268-294$.

Deutsch, M., \& Gerard, H. B. (1955). A study of normative and informational social influences upon individual judgment. Journal of Abnormal and Social Psychology, 51, $629-636$.

Dodd, D. H., \& Bradshaw, J. M. (1980). Leading questions and memory: Pragmatic constraints. Journal of Verbal Learning and Verbal Behavior, 19, 695 - 704. 
Dunning, D., Johnson, K., Ehrlinger, J., \& Kruger, J. (2003). Why people fail to recognize their own incompetence. Current Directions in Psychological Science, $12,83-87$.

Echterhoff, G., Higgins, E. T., \& Groll, S. (2005). Audience-tuning effects on memory: The role of shared reality. Journal of Personality and Social Psychology, 89, $257-276$.

Echterhoff, G., Hirst, W., \& Hussy, W. (2005). How eyewitnesses resist misinformation: Social postwarnings and the monitoring of memory characteristics. Memory and Cognition, 33, $770-782$.

Edwards, D., \& Middleton, D. (1986). Joint remembering: Constructing an account of shared experience through conversational discourse. Discourse Processes, 9, 423 - 459.

Edwards, D., \& Middleton, D. (1987). Conversation and remembering: Bartlett revisited. Applied Cognitive Psychology, 1, 77 - 92.

Edwards, D., \& Middleton, D. (1988). Conversational remembering and family relationships: How children learn to remember. Journal of Social and Personal Relationships, 5, 3 - 25.

Finlay, F., Hitch, G. J., \& Meudell, P. R. (2000). Mutual inhibition in collaborative recall: Evidence for a retrieval-based account. Journal of Experimental Psychology: Learning, Memory and Cognition, 26, 1556 - 1567.

Fivush, R. (1991). The social construction of personal narratives. Merrill-Palmer Quarterly, 37, $59-82$.

Fivush, R., Haden, C., \& Reese, E. (1996). Remembering, recounting, and reminiscing: The development of autobiographical memory in social context. In D. C. Rubin (Ed.). Reconstructing our past: An overview of autobiographical memory (pp. 341 - 359). Cambridge, MA: Cambridge University Press.

French, L., Sutherland, R., \& Garry, M. (2006). Discussion affects memory for true and false childhood events. Applied Cognitive Psychology, 20, 671 - 680. 
Gabbert, F., Memon, A., \& Allan, K. (2003). Memory conformity: Can eyewitnesses influence each other's memories for an event? Applied Cognitive Psychology, 17, 533 - 543.

Gabbert, F., Memon, A., Allan, K., \& Wright, D. B. (2004). Say it to my face: Examining the effects of socially encountered misinformation. Legal and Criminological Psychology, 9, 215 - 227.

Gabbert, F., Memon, A., \& Wright, D. B. (2006). Memory conformity: Disentangling the steps towards influence during a discussion. Psychonomic Bulletin and Review, 13, $480-485$.

Gabbert, F., Memon, A., \& Wright, D. B. (2007). I saw it for longer than you: The relationship between perceived encoding duration and memory conformity. Acta Psychologica, 124, 319 - 331.

Gilbert, D. T., Pelham, B. W., \& Krull, D. S. (1988). On cognitive busyness: When person perceivers meet persons perceived. Journal of Personlaity and Social Psychology, 544, 733 - 740.

Gottman, J. M., Coan, J., Carrere, S., \& Swanson, C. (1998). Predicting marital happiness and stability from newlywed interactions. Journal of Marriage and the Family, 6o, $5-22$.

Granhag, P. A., Ask, K., \& Rebelius, A. (2005, June). "I saw the man who killed Anna Lindh" A case study of witnesses' offender descriptions. Paper presented at the European Law and Psychology Meeting, Vilnuis, Lithuania.

Griffin, D., \& Gonzalez, R. (1995). Correlational analysis of dyad-level data in the exchangeable case. Psychological Bulletin, 118, $430-439$.

Halbwachs, M. (1980). The collective memory (pp. 22 - 49). NY: Harper and Row.

Hardin, C. D., \& Higgins, E. T. (1996). Shared reality: How social verification makes the subjective objective. In R. M. Sorrentino \& E. T. Higgins (Eds.). Handbook of motivation and cognition: Vol. 3. The interpersonal context (pp. 29-84). New York: The Guilford Press. 
Hewitt, P. G. (2001). Conceptual physics ( $9^{\text {th }}$ ed, pp. $\left.576-581\right)$. San Francisco: Addison Wesley.

Hicks, J. L., \& Cockman, D. W. (2003). The effect of general knowledge on source memory and decision processes. Journal of Memory and Language, 48 , $489-501$.

Highhouse, S., \& Bottrill, K. V. (1995). The influence of social (mis)information on memory for behavior in an employment interview. Organizational Behavior and Human Decision Processes, 62, 220 - 229.

Hirokawa, K., Matsuno, E., Mori, K., \& Ukita, J. (2006). Relationship between masculinity-femininity and concession in an experimental collaborative eyewitness testimony. Asian Journal of Social Psychology, 9, 132 - 139.

Hoffman, H. G., Granhag, P. A., See, S., Kwong, T., \& Loftus, E. F. (2001). Social influences on reality-monitoring decisions. Memory and Cognition, 29, 394 -404 .

Hollin, C. R., \& Clifford, B. R. (1983). Eyewitness testimony: The effects of discussion on recall accuracy and agreement. Journal of Applied Social Psychology, 13, $234-244$.

Hollingshead, A. B. (1998a). Communication, learning, and retrieval in transactive memory systems. Journal of Experimental and Social Psychology, 34, 423-442.

Hollingshead, A. B. (1998b). Retrieval processes in transactive memory systems. Journal of Personality and Social Psychology, 74, $659-671$.

Hollingshead, A. B. (200o). Perceptions of expertise and transactive memory in work relationships. Group Processes and Intergroup Relations, 3, $257-267$.

Hollingshead, A. B., \& Fraidin, S. (2003). Gender stereotypes and assumptions about expertise in transactive memory. Journal of Experimental Social Psychology, 39, $355-363$. 
Holtgraves, T., Srull, T. K., \& Socall, D. (1989). Conversation memory: The effects of speaker status on memory for the assertiveness of conversation remarks. Journal of Personality and Social Psychology, 56, 149 - 16o.

Hood, L. (2001). A city possessed. The Christchurch civic crèche case: Child abuse, gender politics and the law. Dunedin, New Zealand: Longacre Press.

Hope, L., Ost, J., Gabbert, F., Healey, S., \& Lenton, E. (2008). "With a little help from my friends...”: The role of co-witness relationship in susceptibility to misinformation. Acta Psychologica.

Hyman, I. E., Jr., \& Faries, J. M. (1992). The functions of autobiographical memory. In M. A. Conway, D. C. Rubin, H. Spinnler, \& W. A. Wagenaar (Eds.). Theoretical perspectives on autobiographical memory (pp. 207 221). Netherlands: Kluwer Academic Publishers.

Hyman, I. E., Jr., \& Loftus, E. F. (1998). Errors in autobiographical memory. Clinical Psychology Review, 18, 933 - 947.

Ikier, S., Tekcan, A. I., Gulgoz, S., \& Kuntay, A. C. (2003). Whose life is it anyway? Adoption of each other's autobiographical memories by twins. Applied Cognitive Psychology, 17, 237 - 247.

Itoh, Y. (2006, July). The effect of cooperative remembering on accuracy on a subsequent memory test: Analysis of conversation. Paper presented at the $4^{\text {th }}$ International Conference on Memory, Sydney, Australia.

Itoh, Y., Umeda, S., \& Kawaguchi, J. (2005, January). The effect of warning against conformity on memory accuracy after cooperative remembering. Paper presented at the $6^{\text {th }}$ biennial conference for the Society of Applied Research in Memory and Cognition, Wellington, New Zealand.

Itsukushima, Y., Nishi, M., Maruyama, M., \& Takahashi, M. (2006). The effect of presentation medium of post-event information: Impact of co-witness information. Applied Cognitive Psychology, 20, 575 - 581.

Johnson, M. K., Bush, J. G., \& Mitchell, K. J. (1998). Interpersonal reality monitoring: Judging the sources of other people's memories. Social Cognition, 16, 199 - 224. 
Johnson, M. K., Foley, M. A., Suengas, A. G., \& Raye, C. L. (1988). Phenomenal characteristics of memories for perceived and imagined autobiographical events. Journal of Experimental Psychology: General, 117, 371 - 376.

Johnson, M. K., Hashtroudi, S., \& Lindsay, D. S. (1993). Source monitoring. Psychological Bulletin, 114, 3 - 28.

Kanematsu, H., Mori, K., \& Mori, H. (2003). Memory distortion in eyewitness pairs who observed nonconforming events and discussed them. Journal of Faculty of Education, Shinshu University, 109, 75 - 84.

Kashy, D. A., \& Kenny, D. A. (200o). The analysis of data from dyads and groups. In H. T. Reis \& C. M. Judd (Eds.). Handbook of research methods in social and personality psychology (pp. 451 - 477). Cambridge: Cambridge University Press.

Kassin, S. M., \& Kiechel, K. L. (1996). The social psychology of false confessions: Compliance, internalization, and confabulation. Psychological Science, 7, $125-128$.

Kenny, D. A. (1996). Models of non-independence in dyadic research. Journal of Social and Personal Relationships, 13, 279 - 294.

Kenny, D. A., Kashy, D. A., \& Cook, W.L. (20o6). Dyadic Data Analysis (pp $32-40$ ). New York: The Guilford Press.

Kowner, R., \& Wiseman, R. (2003). Culture and status-related behavior: Japanese and American perceptions of interaction in asymmetric dyads. CrossCultural Research, 37, $178-210$.

Kraemer, H. C., \& Jacklin, C. N. (1979). Statistical analysis of dyadic social behavior. Psychological Bulletin, 86, 217 - 224.

Kronlund, A. \& Whittlesea, B. W. A. (2005). Seeing double: Levels of processing can cause false memory. Canadian Journal of Experimental Psychology, $59,11-16$. 
Kruger, J., \& Dunning, D. (1999). Unskilled and unaware of it: How difficulties in recognizing one's own incompetence lead to inflated self-assessments. Journal of Personality and Social Psychology, 77, 1121 - 1134.

Lalwani, A. K., Shavitt, S., \& Johnson, T. (2006). What is the relationship between cultural orientation and socially desirable responding? Journal of Personality and Social Psychology, 9o, 165 - 178.

Laney, C., Kaasa, S. O., Morris, E. K., Berkowitz, S. R., Bernstein, D. M., \& Loftus, E. F. (2008). The Red Herring technique: A methodological response to the problem of demand characteristics. Psychological Research. First published online 4 August 2007, DOI: 10.1007/soo426-007-0122-6

Larson, J. R., Jr., Foster-Fishman, P. G., \& Franz, T. M. (1998). Leadership style and the discussion of shared and unshared information in decision-making groups. Personality and Social Psychology Bulletin, 24, 482 - 495.

Larson, J. R., Jr., Foster-Fishman, P. G., \& Keys, C. B. (1994). Discussion of shared and unshared information in decision-making groups. Journal of Personality and Social Psychology, 67, 446 - 461.

Latané, B., Williams, K., \& Harkins, S. (1979). Many hand make light the work: The causes and consequences of social loafing. Journal of Personality and Social Psychology, 37, $822-832$.

Lindsay, D. S. (1990). Misleading suggestions can impair eyewitnesses' ability to remember event details. Journal of Experimental Psychology: Learning, Memory and Cognition, 16, 1077 - 1083.

Lindsay, D. S. (2007). Order effects in collaborative memory contamination? Comment on Gabbert, Memon, and Wright (2006). Psychonomic Bulletin and Review, 14, 1010.

Lindsay, D. S. (2008). Source monitoring. In J. Byrne (Series Ed.) \& H. L. Roediger, III (Vol. Ed.). Cognitive psychology of memory: Vol. 2. Learning and memory: A comprehensive reference. Oxford: Elsevier.

Loftus, E. F. (1975). Leading questions and the eyewitness report. Cognitive Psychology, 7, 560 - 572. 
Loftus, E. F. (1991). Made in memory: Distortions in recollection after misleading information. In G.H. Bower (Ed.). The psychology of learning and motivation: Advances in research and theory (pp. 187-215). San Diego, CA: Academic Press.

Loftus, E. F., Ledivow, B., \& Duensing, S. (1992). Who remembers best? Individual differences in memory for events that occurred in a science museum. Applied Cognitive Psychology, 6, 93 - 107.

Loftus, E. F., Miller, D. G., \& Burns, H. J. (1978). Semantic integration of verbal information into visual memory. Journal of Experimental Psychology: Human Learning and Memory, 4, 19 - 31.

Loftus, E. F., \& Palmer, J. C. (1974). Reconstruction of automobile deconstruction: An example of the interaction between language and memory. Journal of Verbal Learning and Verbal Behavior, 13, 585-589.

Loftus, E. F., \& Zanni, G. (1975). Eyewitness testimony: the influence of the wording of a question. Bulletin of the Psychonomic Society, 5, 86 - 88.

Loftus, G. R. (1996). Psychology will be a much better science when we change the way we analyze data. Current Directions in Psychological Science, 5, $161-171$.

Luus, C. A. E., \& Wells, G. L. (1994). The malleability of eyewitness confidence: Co-witness and perseverance effects. Journal of Applied Psychology, 79, $714-723$.

MacDonald, S., Uesiliana, K., \& Hayne, H. (2000). Cross-cultural and gender differences in childhood amnesia. Memory, 8, 365-376.

Marsh, E. J. (2007). Retelling is not the same as recalling: Implications for memory. Current Directions in Psychological Science, 16, 16 - 20.

Marsh, E. J. \& Tversky, B. (2004). Spinning the stories of our lives. Applied Cognitive Psychology, 18, 491 - 503. 
Mather, M., Johnson, M. K., \& De Leonardis, D. M. (1999). Stereotype reliance in source monitoring: Age differences and neuropsychological test correlates. Cognitive Neuropsychology, 16, $437-45^{8}$.

McCloskey, M., \& Zaragoza, M. (1985). Misleading postevent information and memory for events: Arguments and evidence against memory impairment hypotheses. Journal of Experimental Psychology: General, $114,1-16$.

Meade, M. L., \& Roediger, H. L., III. (2002). Explorations in the social contagion of memory. Memory and Cognition, 30, 995 - 1009.

Memon, A., \& Wright, D. B. (1999). Eyewitness testimony and the Oklahoma bombing. The Psychologist, 12, $292-295$.

Meudell, P. R., Hitch, G. J., \& Kirby, P. (1992). Are two heads better than one? Experimental investigations of the social facilitation of memory. Applied Cognitive Psychology, 6, 525 - 543.

Mitchell, K. J., \& Johnson, M. K. (2000). Source monitoring: Attributing mental experiences. In E. Tulving \& F. I. M. Craik (Eds.). The Oxford handbook of memory (pp. 179 - 195). New York: Oxford University Press.

Mori, K. (2003). Surreptitiously projecting different movies to two subsets of viewers. Behavior Research Methods, Instruments, and Computers, 35, 599 -604 .

Mori, K. (2005, January). A new experimental paradigm for collaborative eyewitness testimony using a presentation trick. Paper presented at the $6^{\text {th }}$ biennial conference for the Society of Applied Research in Memory and Cognition, Wellington, New Zealand.

Mori, K. (2007). A revised method for projecting two different movies to two groups of viewers without their noticing the duality. Behavior Research Methods, 39, $574-578$. 
Mori, K., \& Kitabayashi, M. (2006, July). Interpersonal memory dynamics among child-mother eyewitness pairs: An experimental examination using the MORI technique. Paper presented at the $4^{\text {th }}$ International Conference on Memory, Sydney, Australia.

Mori, K., \& Mori, H. (2006). Second-order effects of the presence of co-witnesses on memory conformity in experimental collaborative eyewitness testimony. Unpublished manuscript.

Neisser, U. (1988). Five kinds of self-knowledge. Philosophical Psychology, 1, 35 59.

Neisser, U. \& Harsh, N. (1992). Phantom flashbulbs: False recollections of hearing the news about Challenger. In E. Winograd \& U. Neisser (Eds.). Affect and accuracy in recall: Studies of "flashbulb" memories. Emory symposia in cognition, Vol. 4. (pp. 9 - 31). New York: Cambridge University Press.

Nelson, K. (2003). Self and social functions: Individual autobiographical memory and collective narrative. Memory, 11, $125-136$.

Parker, S., Garry, M., Engle, R. W., Harper, D. N., \& Clifasefi, S. L. (2008). Psychotropic placebos reduce the misinformation effect by increasing monitoring at test. Memory.

Paterson, H., Chapman, L., \& Kemp, R. (2007, July). The effects of false memory feedback on susceptibility to co-witness misinformation. Paper presented at the 3rd International Congress of Psychology and Law, Adelaide, Australia.

Paterson, H. M., \& Kemp, R. I. (2006a). Co-witnesses talk: A survey of eyewitness discussion. Psychology, Crime and Law, 12, 181 - 191.

Paterson, H. M., \& Kemp, R. I. (2006b). Comparing methods of encountering postevent information: The power of co-witness suggestion. Applied Cognitive Psychology, 20, 1083 - 1099.

Pickel, K. L. (1993). Evaluation and integration of eyewitness reports. Law and Human Behavior, 17, 569 - 595. 
Principe, G. F., \& Ceci, S. J. (2002). "I saw it with my own ears": The effects of peer conversations on preschoolers reports on nonexperienced events. Journal of Experimental Child Psychology, 83, 1 - 25.

Reese, E., \& Brown, N. (200o). Reminiscing and recounting in the preschool years. Applied Cognitive Psychology, 14, 1 - 17.

Reysen, M. B. (2005). The effects of conformity on recognition judgments. Memory, 13, $87-94$.

Roediger, H. L., III, Meade, M. L., \& Bergman, E. T. (2001). Social contagion of memory. Psychonomic Bulletin and Review, 8, 365- 371.

Ross, M. (1997). Validating memories. In N. L. Stein, P. A. Ornstein, B. Tversky, \& C. Brainerd (Eds.). Memory for everyday and emotional events (pp. 49 82). Hillsdale, NJ: Erlbaum.

Ross, M., \& Conway, M. (1986). Remembering one's own past: The construction of personal histories. In R. M. Sorrentino \& E. T. Higgins (Eds.). Handbook of motivation and cognition: Foundations of social behavior (pp. 122 - 144). NY: Guilford Press.

Schneider, D. M., \& Watkins, M. J. (1996). Response conformity in recognition testing. Psychonomic Bulletin and Review, 3, 481 - 485 .

Shaw, J. S., Garven, S., \& Wood, J. M. (1997). Co-witness information can have immediate effects on eyewitness memory reports. Law and Human Behavior, 21, $503-523$.

Sheen, M., Kemp, S., \& Rubin, D. (2001). Twins dispute memory ownership: A new false memory phenomenon. Memory and Cognition, 29, 779 - 788.

Skagerberg, E. M., \& Wright, D. B. (2008). Manipulating power can affect memory conformity. Applied Cognitive Psychology, 22, 207 - 216.

Smith, V. L., \& Ellsworth, P. C. (1987). The social psychology of eyewitness accuracy: Misleading questions and communicator expertise. Journal of Applied Psychology, 72, 294 - 300. 
Stasser, G. (1999). The uncertain role of unshared information in collective choice. In L. L. Thompson, J. M. Levine, \& D. M. Messick (Eds.). Shared cognition in organizations: The management of knowledge (pp. 49 -69). Mahwah, NJ: Lawrence Erlbaum.

Stasser, G., Stewart, D. D., \& Wittenbaum, G. M. (1995). Expert roles and information exchange during discussion: The importance of knowing who knows what. Journal of Experimental Social Psychology, 31, $244-265$.

Stephenson, G. M., Kniveton, B. H., \& Wagner, W. (1991). Social influences on remembering: Intellectual, interpersonal, and intergroup components. European Journal of Social Psychology, 21, 463-475.

Stewart, D. D., \& Stasser, G. (1995). Expert role assignment and information sampling during collective recall and decision making. Journal of Personality and Social Psychology, 69, $619-682$.

Sun, T., Horn, M., \& Merritt, D. (2004). Values and lifestyles of individualists and collectivists: A study on Chinese, Japanese, British and US consumers. Journal of Consumer Marketing, 21, 318 - 331.

Swedish minister dies after stabbing. (2003, 11 September). $B B C$ News. Retrieved April 3, 2008 from http://news.bbc.co.uk/1/hi/world/europe/3098834.stm

Takahashi, M., \& Saito, S. (2004). Does test delay eliminate collaborative inhibition? Memory, 12, $722-731$.

Takarangi, M. K. T., Parker, S. L., \& Garry, M. (2006). Modernising the misinformation effect: The development of a new stimulus set. Applied Cognitive Psychology, 20, $1-8$.

Thomas, J. (1997a, May 12). Swift, hard attack in bombing trial. New York Times. Retrieved April 3, 2008 from http://query.nytimes.com/gst/fullpage.html?res=9Fo2EoD61339F931A25756C oA96195826o\&sec=\&spon=\&pagewanted $=$ all 
Thomas, J. (1997b, January 30). Suspect's sketch in Oklahoma case called an error. New York Times. Retrieved April 3, 2008 from http://query.nytimes.com/gst/fullpage.html?res=9Co5E2DC173DF933Ao5752 CoAg6195826o\&scp=3\&sq=Tom + Kessinger + witness\&st $=$ nyt $\#$

Thomas, J. (1997c, February 19). Truck was rented by Oklahoma bomb suspect, witnesses say. New York Times. Retrieved April 3, 2008 from http://query.nytimes.com/gst/fullpage.html?res=9FooE7DF163EF93AA25751 CoA96195826o\&sec $=\&$ spon $=\&$ pagewanted $=$ all

Thomas, J. (1997d, June 15). Still looking for answers. New York Times. Retrieved April 3, 2008 from http://query.nytimes.com/gst/fullpage.html?res=9504E6DD103FF936A25755 CoAg6195826o\&sec $=\&$ spon $=\&$ pagewanted $=$ all

Thompson, B. (2002). What future quantitative social science research could look like: Confidence intervals for effect sizes. Educational Researcher, 31, 25 32.

Tousignant, J. P., Hall, D., \& Loftus, E. F. (1986). Discrepancy detection and vulnerability to misleading postevent information. Memory and Cognition, 14, 329 - 338.

Underwood, G., \& Milton, H. (1993). Collusion after a collision: witnesses' reports of a road accident with and without discussion. Applied Cognitive Psychology, 7, $11-22$.

Underwood, J., \& Pezdek, K. (1998). Memory suggestibility as an example of the sleeper effect. Psychonomic Bulletin and Review, 5, 449 - 453.

Vornik, L. A., Sharman, S. J., \& Garry, M. (2003). The power of the spoken word: Sociolinguistic cues influence the misinformation effect. Memory, 11, $101-$ 109.

Wade-Benzoni, K. A., Okumura, T., Brett, J. M., Moore, D. A., Tenbrunsel, A. E., \& Bazerman, M. H. (2002). Cognitions and behavior in asymmetric social dilemmas: A comparison of two cultures. Journal of Applied Psychology, $87,87-95$. 
Walther, E., Bless, H., Strack, F., Rackstraw, P., Wagner, D., \& Werth, L. (2002). Conformity effects in memory as a function of group size, dissenters and uncertainty. Applied Cognitive Psychology, 16, 793 - 810.

Warnick, D. H., \& Sanders, G. S. (1980). The effects of group discussion on eyewitness accuracy. Journal of Applied Social Psychology, 10, 249 - 259.

Wegner, D. M. (1987). Transactive memory: A contemporary analysis of the group mind. In B. Mullen \& G. R. Goethals (Eds.). Theories of group behavior (pp. 185 - 208). NY: Springer-Verlag.

Wegner, D. M., Erber, R., \& Raymond, P. (1991). Transactive memory in close relationships. Journal of Personality and Social Psychology, 61, 923-929.

Wegner, D. M., Giuliano, T., \& Hertel, P. T. (1985). Cognitive interdependence in close relationships. In W. J. Ickes (Ed.) Compatible and incompatible relationships (pp. 253 - 276). New York: Springer-Verlag.

Weldon, M. S. (2001). Remembering as a social process. The Psychology of Learning and Motivation, 40, $67-120$.

Weldon, M. S., \& Bellinger, K. D. (1997). Collective memory: Collaborative and individual processes in remembering. Journal of Experimental Psychology: Learning, Memory, and Cognition, 23, 1160 - 1175.

Weldon, M. S., Blair, C., \& Huebsch, P. D. (2000). Group remembering: Does social loafing underlie collaborative inhibition? Journal of Experimental Psychology: Learning, Memory and Cognition, 26, 1568 - 1577.

Wittenbaum, G. M. (1998). Information sampling in decision-making groups: The impact of members' task-relevant status. Small Group Research, 29, 57 84 .

Wright, D. B., \& Klumpp, A. (2004). Collaborative inhibition is due to the product, not the process, of recalling in groups. Psychonomic Bulletin and Review, $11,1080-1083$. 
Wright, D. B., Mathews, S. A., \& Skagerberg, E. M. (2005). Social recognition memory: The effect of other people's responses for previously seen and unseen items. Journal of Experimental Psychology: Applied, 11, 200 - 209.

Wright, D. B., Self, G., \& Justice, C. (2000). Memory conformity: Exploring misinformation effects when presented by another person. British Journal of Psychology, 91, $189-202$.

Wyer, R. S., \& Gruenfeld, D. H. (1995). Information processing in social contexts: Implications for social memory and judgment. Advances in Experimental Social Psychology, 27, 49 - 91.

Yarmey, A. D. (1992). The effects of dyadic discussion on earwitness recall. Basic and Applied Social Psychology, 13, $251-263$.

Yarmey, A. D., \& Morris, S. (1998). The effects of discussion on eyewitness memory. Journal of Applied Social Psychology, 28, 1637 - 1648. 


\section{Appendix A: Sample Misinformation Narrative}

(Experiments $1 \mathrm{~A}$ and $1 \mathrm{~B}$ )

One afternoon, Eric Lewis, an average looking (although dishonest) guy with brown hair, had a job at a house in the city. Eric pulled up to the house in his blue "AJ's Electricians" van and parked in the driveway. After getting his tool belt and drill kit out of the van, Eric went to the front door and retrieved the key that was left for him from under a flowerpot.

He unlocked the door and walked into the house, where he found a note from the homeowner on the hallstand, next to a vase of flowers. After reading the note, Eric put it in his pocket and put his drill kit down. Although being an electrician could get boring, one thing Eric liked about his job was that he could be nosy. He walked into a bedroom on the right, past the bed with its bright pink bedspread, and over to the dresser. He noticed the dresser was covered with a navy cloth. Curious, Eric bent down to lift it up and investigate the drawers underneath. Seeing nothing very interesting, Eric checked out the items on the dresser, including a wooden jewellery box. He opened the box and removed a pair of earrings, which he inspected carefully and-thinking of his girlfriend's upcoming birthday_slipped them into his pocket.

Eric headed down the hallway to the living area, picking up his drill on the way. He thought the house was getting a little hot and stuffy, so he opened the French doors in the lounge and went into the adjacent kitchen. He put his drill kit down on the bench and thought he was feeling hungry, so he helped himself to a can of pepsi from the fridge and an apple from the fruit bowl. He continued to look around the kitchen, finding nothing interesting in the pantry. Always curious about the secret lives of his customers, Eric rummaged through a pile of papers next to a mug on the kitchen bench, but there wasn't much interesting there either.

Thinking he should get down to work, Eric walked over to the broken oven and bent down to examine it. He removed a screwdriver from his tool belt and repaired the front panel. His kitchen repair finished, Eric headed down the hallway again to the second bedroom. In the bedroom, he kneeled down to check one of the power points that the homeowners said was not working. Once that was done, Eric got up to have a look around the room. He tried on a blue cap and checked his reflection in the mirror, but he didn't really like the way it looked on him. After replacing the cap on the bed, he browsed through the wardrobe, but 
didn't see anything he liked. Eric sat down on the bed to read a news magazine, but found it boring, and tossed it back on the floor. Looking up, he spotted a silver ring next to the stereo and tried it on. Thinking it looked pretty good on him, he pocketed the ring and started to look through a pile of CDs on the stereo. After selecting a CD he knew he would enjoy, Eric went back into the lounge to play it on the stereo in there, thinking he would listen to it while he finished his work in the lounge. His last job was a light fitting on the lounge wall. After removing the light cover, and working on the wiring, Eric flicked the switch on and off but nothing happened. He adjusted things a bit and the light finally came on.

Now that his work was completed, Eric decided that he deserved to relax for a while. He sat down on the couch, turned off the stereo and-finding the black remote on the coffee table in front of him-switched on the TV. He picked up a red photo album that was lying on the wooden coffee table and flicked through it. After a while, he checked his watch. Realizing he needed to get to his next job, Eric got up and turned the TV off. He retrieved the CD from the stereo and, thinking it would be good to listen to back in the van, he put it in his drill kit. After shutting and locking the French doors, he stopped to look at a picture on the wall. On his way out, Eric decided to have a quick look through the bathroom cabinet, where he pocketed some prescription pills that he thought he might be able to sell. By now, he was late for his next job, so Eric hurried to the door and closed it behind him as he left. 


\section{Appendix B: Example Discussion Questions for Eric Materials}

(Experiments $2 \mathrm{~A}$ and 4 )

The walls in the hallway were painted:

Cream $\quad$ Red Green Brown Blue

In the kitchen, Eric fixed the:

Dishwasher

Oven

Microwave

Extractor fan

Fridge

Eric got the remote from the:

Television Kitchen bench

Floor

Coffee table

Couch

The name of Eric's company was:

AJ's Electricians EJ's Electricians JD's Electricians DJ's Electricians RJ's Electricians

Outside the lounge doors, Eric could see a:

Road

School

Swimming pool

Dog kennel

Lawn

Eric drank a can of:

Fanta

Coke

Export gold

Pepsi

Sprite

Eric left his drill and drill case:

On the In the driveway In the hallway In the first In his van doorstep bedroom

The curtains in the lounge were:

\begin{tabular}{ccccc} 
Brown & White & Blue & Patterned & Green \\
\hline Eric tried on a & cap: & & & \\
Blue & Black & Green & Red & Grey \\
\hline
\end{tabular}

Eric stole a CD by hiding it in his:

Pocket

Van

Bag

Hat

Drill case

In the lounge, Eric sat on a couch:

Purple

Red

Green

White

Brown

Eric checked the time:

On his cellphone
On the microwave
On the video player
On his watch

On his watch

On the wall clock 


\section{Appendix G: Recognition Test for Eric Materials}

(Experiments $1 \mathrm{~A},{ }_{1} \mathrm{~B}, 2 \mathrm{~A}$, and 4 )

You will now be asked some questions about the video you saw. We are testing your memory for this video.

Each question has two parts:

1) the first part asks you about a particular item from the video;

2) the second part asks you how confident you are about your answer.

Here is a sample question.

Eric was working in
a. a house
b. a shop

How confident are you that your answer is correct?

1

Not at all

Confident
2

3

4

5

Very

Confident

WHEN YOU HAVE READ AND UNDERSTOOD HOW TO ANSWER THESE QUESTIONS, TURN OVER THE PAGE AND BEGIN THE TEST. 
1. Eric was wearing
a. overalls
b. jeans

How confident are you that your answer is correct?

\begin{tabular}{|c|c|c|c|}
\hline $\begin{array}{l}1 \\
\text { Not at all } \\
\text { Confident }\end{array}$ & 2 & 3 & 4 \\
\hline
\end{tabular}

2. Eric ate
a. an apple
b. a banana

How confident are you that your answer is correct?

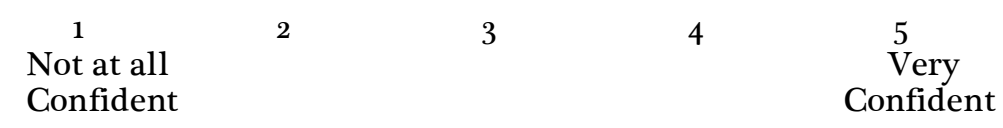

3. The magazine that Eric read was
a. Time
b. Newsweek

How confident are you that your answer is correct?

$\begin{array}{lllll}1 & 2 & 3 & 4 & 5 \\ \begin{array}{l}\text { Not at all } \\ \text { Confident }\end{array} & & & & \begin{array}{c}\text { Very } \\ \text { Confident }\end{array}\end{array}$

4. Eric read the note from the homeowner in the
a. kitchen
b. hallway

How confident are you that your answer is correct?

\begin{tabular}{|c|c|c|c|c|}
\hline $\begin{array}{c}1 \\
\text { Not at all } \\
\text { Confident }\end{array}$ & 2 & 3 & 4 & $\begin{array}{c}5 \\
\text { Very } \\
\text { Confident }\end{array}$ \\
\hline
\end{tabular}

5. The tool that Eric used in the kitchen was
a. pliers
b. a screwdriver

How confident are you that your answer is correct?

$\begin{array}{llllc}1 & 2 & 3 & 4 & 5 \\ \text { Not at all } & & & & \begin{array}{c}\text { Very } \\ \text { Confident }\end{array}\end{array}$


6. In the lounge the picture Eric looked at was the Tower
a. Eiffel
b. Leaning

How confident are you that your answer is correct?

\begin{tabular}{|c|c|c|c|c|}
\hline $\begin{array}{l}1 \\
\text { Not at all } \\
\text { Confident }\end{array}$ & 2 & 3 & 4 & $\begin{array}{c}5 \\
\text { Very } \\
\text { Confident }\end{array}$ \\
\hline
\end{tabular}

7. The bed in the first bedroom was
a. made
b. unmade

How confident are you that your answer is correct?

$\begin{array}{llllc}1 & 2 & 3 & 4 & 5 \\ \text { Not at all } & & & & \text { Very } \\ \text { Confident } & & & & \text { Confident }\end{array}$

8. In the second bedroom, Eric tested a
a. power point
b. light fitting

How confident are you that your answer is correct?

$\begin{array}{llllc}1 & 2 & 3 & 4 & 5 \\ \text { Not at all } & & & & \begin{array}{c}\text { Very } \\ \text { Confident }\end{array} \\ & & & & \end{array}$

9. Eric played a
a. video
b. CD

How confident are you that your answer is correct?

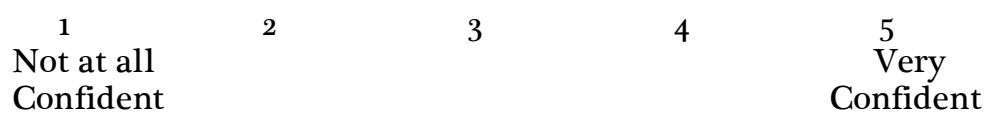

10. In the second bedroom, Eric tried on a cap
a. red
b. black

How confident are you that your answer is correct?

\begin{tabular}{|c|c|c|c|}
\hline $\begin{array}{c}1 \\
\text { Not at all } \\
\text { Confident }\end{array}$ & 2 & 3 & 4 \\
\hline
\end{tabular}


11. The name of Eric's company was

\section{a. AJ's Electricians b. RJ's Electricians}

How confident are you that your answer is correct?

$\begin{array}{llllc}1 & 2 & 3 & 4 & 5 \\ \begin{array}{l}\text { Not at all } \\ \text { Confident }\end{array} & & & & \begin{array}{c}\text { Very } \\ \text { Confident }\end{array}\end{array}$

12. Eric checked the time
a. on his watch
b. on the wall clock

How confident are you that your answer is correct?

$\begin{array}{llllc}1 & 2 & 3 & 4 & 5 \\ \begin{array}{l}\text { Not at all } \\ \text { Confident }\end{array} & & & & \text { Very } \\ \text { Confident }\end{array}$

13. The jewellery that Eric stole in the first bedroom was
a. earrings
b. a necklace

How confident are you that your answer is correct?

$\begin{array}{llllc}1 & 2 & 3 & 4 & 5 \\ \text { Not at all } & & & & \text { Very } \\ \text { Confident } & & & & \end{array}$

14. In the lounge Eric looked through a
a. journal
b. photo album

How confident are you that your answer is correct?

\begin{tabular}{|c|c|c|c|c|}
\hline $\begin{array}{c}1 \\
\text { Not at all } \\
\text { Confident }\end{array}$ & 2 & 3 & 4 & $\begin{array}{c}5 \\
\text { Very } \\
\text { Confident }\end{array}$ \\
\hline
\end{tabular}

15. Eric's van was
a. blue
b. red

How confident are you that your answer is correct?

$\begin{array}{llllc}1 & 2 & 3 & 4 & 5 \\ \text { Not at all } & & & & \begin{array}{c}\text { Very } \\ \text { Confident }\end{array}\end{array}$


16. Eric found the house key under a
a. door mat
b. flower pot

How confident are you that your answer is correct?

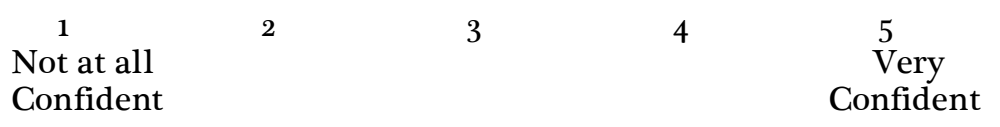

17. Eric rummaged through papers that were next to a mug
a. green
b. white

How confident are you that your answer is correct?

$\begin{array}{llccc}1 & 2 & 3 & 4 & 5 \\ \begin{array}{l}\text { Not at all } \\ \text { Confident }\end{array} & & & & \begin{array}{c}\text { Very } \\ \text { Confident }\end{array}\end{array}$

18. Eric drank a can of
a. coke
b. pepsi

How confident are you that your answer is correct?

\begin{tabular}{|c|c|c|c|c|}
\hline $\begin{array}{c}1 \\
\text { Not at all } \\
\text { Confident }\end{array}$ & 2 & 3 & 4 & $\begin{array}{c}5 \\
\text { Very } \\
\text { Confident }\end{array}$ \\
\hline
\end{tabular}

19. In the bathroom Eric stole
a. pills
b. perfume

How confident are you that your answer is correct?

\begin{tabular}{|c|c|c|c|c|}
\hline $\begin{array}{c}1 \\
\text { Not at all } \\
\text { Confident }\end{array}$ & 2 & 3 & 4 & $\begin{array}{c}5 \\
\text { Very } \\
\text { Confident }\end{array}$ \\
\hline
\end{tabular}

20. Eric stole in the second bedroom
a. money
b. a ring

How confident are you that your answer is correct?

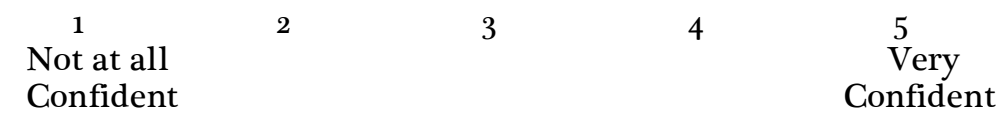




\section{Appendix D: Example Discussion Questions for Chad Materials}

(Experiments ${ }_{2} \mathrm{~B}, 5$ and 6 )

How many people did Chad talk to during the party?

Eight Nine Ten Eleven Twelve

What number was the house that the party was at?

$\begin{array}{lllll}2 \mathrm{~A} & 5 \mathrm{~A} & 12 \mathrm{~A} & 22 \mathrm{~A} & 122 \mathrm{~A}\end{array}$

Where did Chad leave his bag when he got to the party?

Kitchen Hallway Bathroom Bedroom Lounge

What decoration was hanging over the doorway to the lounge?

Tinsel Streamers Happy Birthday Farewell Banner Congratulations Banner Banner

Where did Chad find his cup?

Kitchen cupboard Kitchen bench Table in lounge Lounge window Dishwasher sill

How did Chad get into the house?

$\begin{array}{lcccc}\text { He walked } & \text { He used his } & \text { He made a } & \text { He rang the } & \text { He knocked on } \\ \text { straight in. } & \text { key } & \text { phone call } & \text { doorbell } & \text { the door }\end{array}$

How many times did Chad check his cell phone?

$\begin{array}{llll}\text { One Two } & \text { Three } & \text { Four }\end{array}$

What was Chad wearing?

Button shirt and Button shirt Singlet and $T$ shirt and $T$ shirt and jeans jeans and dress pants shorts shorts

Where did Chad go when he was trying to find the bathroom?

Conservatory Bedroom Linen cupboard Laundry Wardrobe

What colour was the front door?

White

Brown

Cream

Yellow

Green

Where did Chad put the wallet he found on the bench?

In the cupboard Behind the Back where he In his back In his bag microwave found it pocket

What was Chad reaching for when he spilled the drink?
A CD
His drink
Pizza
Chips
Playing cards 


\section{Appendix E: Recognition Test for Chad Materials}

(Experiments $2 \mathrm{~B}, 5$ and 6 )

You will now be asked some questions about the video you saw. We are testing your memory for this video.

Each question has two parts:

1) the first part asks you about a particular item from the video;

2) the second part asks you how confident you are about your answer.

Here is an example question. Circle the correct answer, then rate your confidence using the scale.

What colour was Chad's shirt?
a. Brown
b. Blue

How confident are you that your answer is correct?

Not at all

2

3

4

5

confident confident

WHEN YOU HAVE READ AND UNDERSTOOD HOW TO ANSWER THESE QUESTIONS, TURN OVER THE PAGE AND BEGIN THE TEST. 
1. What sort of bag did Chad bring to the party?
a. Satchel
b. Backpack

How confident are you that your answer is correct?

Not at all

2

3

4

5 confident

2. What was the painting hanging over the drinks table of?
a. Daffodils
b. Sunflowers

How confident are you that your answer is correct?

$\begin{array}{llccc}1 & 2 & 3 & 4 & 5 \\ \text { Not at all } & & & \text { Very } \\ \text { confident } & & & & \text { Confident }\end{array}$

3. Where did Chad go when he was trying to find the toilet?
a. Laundry
b. Bedroom

How confident are you that your answer is correct?

1

Not at all

confident
2

3

4 Very Confident

4. Where was Chad when his cell phone rang?

a. Leaving the kitchen

b. Leaving the bathroom

How confident are you that your answer is correct?

$\begin{array}{lllcc}1 & 2 & 3 & 4 & 5 \\ \begin{array}{l}\text { Not at all } \\ \text { confident }\end{array} & & & & \begin{array}{c}\text { Very } \\ \text { Confident }\end{array}\end{array}$

5. What did Chad sort through at the small table in the lounge?
a. Cutlery
b. CDs

How confident are you that your answer is correct?

$\begin{array}{lllcc}1 & 2 & 3 & 4 & 5 \\ \begin{array}{l}\text { Not at all } \\ \text { confident }\end{array} & & & & \text { Very } \\ \text { Confident }\end{array}$


6. What drink did Chad take out of his brown paper bag?
a. Vodka
b. Wine

How confident are you that your answer is correct?

Not at all

2

3

4

5

confident

Confident

7. What decoration was hanging over the doorway to the lounge?
a. Tinsel
b. Happy Birthday Banner

How confident are you that your answer is correct?

$\begin{array}{llccc}1 & 2 & 3 & 4 & 5 \\ \begin{array}{l}\text { Not at all } \\ \text { confident }\end{array} & & & & \text { Very } \\ \text { Confident }\end{array}$

8. When Chad knocked over the drink, what did he clean up the spill with?
a. Paper towels
b. Tea towel

How confident are you that your answer is correct?

Not at al

23

3

4

confident

Very

Confident

9. What colour were the lounge walls painted?
a. Peach
b. Pink

How confident are you that your answer is correct?

$\begin{array}{ccccc}1 & 2 & 3 & 4 & 5 \\ \begin{array}{l}\text { Not at all } \\ \text { confident }\end{array} & & & & \begin{array}{c}\text { Very } \\ \text { Confident }\end{array}\end{array}$

10. What colour cup did the person who opened the front door have?
a. Pink
b. Blue

How confident are you that your answer is correct?

$\begin{array}{ccccc}1 & 2 & 3 & 4 & 5 \\ \begin{array}{l}\text { Not at all } \\ \text { confident }\end{array} & & & & \begin{array}{c}\text { Very } \\ \text { Confident }\end{array} \\ \end{array}$


11. Where did Chad put the wallet he found on the kitchen bench?
a. In his back pocket
b. Back where he found it

How confident are you that your answer is correct?

$\begin{array}{ccccc}1 & 2 & 3 & 4 & 5 \\ \begin{array}{l}\text { Not at all } \\ \text { confident }\end{array} & & & & \begin{array}{c}\text { Very } \\ \text { Confident }\end{array}\end{array}$

12. What colour balloons were hanging over the bathroom door?
a. Blue
b. Red

How confident are you that your answer is correct?

$\begin{array}{ccccc}1 & 2 & 3 & 4 & 5 \\ \begin{array}{l}\text { Not at all } \\ \text { confident }\end{array} & & & & \begin{array}{c}\text { Very } \\ \text { Confident }\end{array}\end{array}$

13. How many jacket hooks were there beside the drinks table?
a. Four
b. Five

How confident are you that your answer is correct?

$\begin{array}{ccccc}1 & 2 & 3 & 4 & 5 \\ \begin{array}{l}\text { Not at all } \\ \text { confident }\end{array} & & & & \begin{array}{c}\text { Very } \\ \text { Confident }\end{array}\end{array}$

14. What colour was the kitchen bench?
a. Yellow
b. Green

How confident are you that your answer is correct?

$\begin{array}{ccccc}1 & 2 & 3 & 4 & 5 \\ \begin{array}{l}\text { Not at all } \\ \text { confident }\end{array} & & & & \begin{array}{c}\text { Very } \\ \text { Confident }\end{array}\end{array}$

15. What did Chad eat on his way back from the toilet?
a. Chips
b. Carrots

How confident are you that your answer is correct?

$\begin{array}{llccc}1 & 2 & 3 & 4 & 5 \\ \begin{array}{l}\text { Not at all } \\ \text { confident }\end{array} & & & & \begin{array}{c}\text { Very } \\ \text { Confident }\end{array}\end{array}$


16. How many doors did Chad close at the party?
a. Three
b. Four

How confident are you that your answer is correct?

$\begin{array}{lllcc}1 & 2 & 3 & 4 & 5 \\ \begin{array}{l}\text { Not at all } \\ \text { confident }\end{array} & & & & \begin{array}{c}\text { Very } \\ \text { Confident }\end{array}\end{array}$

17. How did Chad get into the house?

a. Knocked on the door $\quad$ b. Rang the doorbell

How confident are you that your answer is correct?

$\begin{array}{ccccc}1 & 2 & 3 & 4 & 5 \\ \begin{array}{l}\text { Not at all } \\ \text { confident }\end{array} & & & & \begin{array}{c}\text { Very } \\ \text { Confident }\end{array}\end{array}$

18. What did Chad add to the drink he made for the woman?
a. A squeeze of lemon
b. A vial of liquid

How confident are you that your answer is correct?

$\begin{array}{ccccc}1 & 2 & 3 & 4 & 5 \\ \begin{array}{l}\text { Not at all } \\ \text { confident }\end{array} & & & & \begin{array}{c}\text { Very } \\ \text { Confident }\end{array}\end{array}$

19. Were the curtains in the lounge closed or open?
a. Closed
b. Open

How confident are you that your answer is correct?

$\begin{array}{lllcc}1 & 2 & 3 & 4 & 5 \\ \begin{array}{l}\text { Not at all } \\ \text { confident }\end{array} & & & & \begin{array}{c}\text { Very } \\ \text { Confident }\end{array}\end{array}$

20. When Chad found it, was the toilet seat up or down?
a. Up
b. Down

How confident are you that your answer is correct?

$\begin{array}{ccccc}1 & 2 & 3 & 4 & 5 \\ \begin{array}{l}\text { Not at all } \\ \text { confident }\end{array} & & & & \begin{array}{c}\text { Very } \\ \text { Confident }\end{array} \\ \end{array}$




\section{Appendix F: Questions in Red Herring Test Booklet}

(Experiment 3A)

1. Do you think Chad is likeable?

2. If you were at the party with Chad, would you have spent time talking to him?

3. If you saw Chad at the party, would you have tried to change his behaviour in any way?

4. Did you notice anything unusual about Chad's behaviour at the party?

5. Did you notice anything unusual about the movie that you watched?

6. Do you think Chad is like most people his age?

7. Would you trust Chad to keep a promise?

8. Would you trust Chad to pay you back money that you had lent him?

9. Do you think the person sitting next to you would share your opinions of Chad?

10. You and another student both watched the movie together-each of you wearing the same kind of acuity glasses-suppose that afterwards I asked you to answer a list of questions about the movie together.

You and the other student agreed on some of the answers and disagreed on others. Please list all the reasons you can think of that would explain why you and the other person did not agree on some of those answers.

11. Many psychology studies use deception. The purpose of deception is to keep subjects from responding in a particular way because they know what the experimenters are looking for.

This study used deception to hide the true nature of the study. That is, we told you this was a study about people's sensory impressions at different levels of visual acuity. By this point in the study, you may have your own theory about what the study was really about. Please use the space below to provide your best guess, and explain your reasoning if you can.

12. Before you saw this question, did you think that you and the person next to you saw the same movie?

13. What did you know about this study before you took part today? 


\section{Appendix G: Second-Ghoice Test}

\section{(Experiment $3 \mathrm{~B})$}

You will now be asked some questions about the movie you saw. We are testing your memory for this movie. Please circle your answers.

Each question has four parts:

1) The first part asks you about a particular item from the movie.

2) The second part asks you how confident you are about your answer.

3) Next you are asked to imagine that your first answer is wrong, and to answer the question again with a second choice. You cannot choose the same answer twice. Circle the answer you think is the next most likely to be correct.

4) Finally we want you to explain, in your own words, why you chose the second answer that you did.

Please answer this example question:

\section{What colour was Chad's hair?}
a. Blonde
b. Brown
c. Black
d. Ginger
e. Grey

How confident are you that your answer is correct?

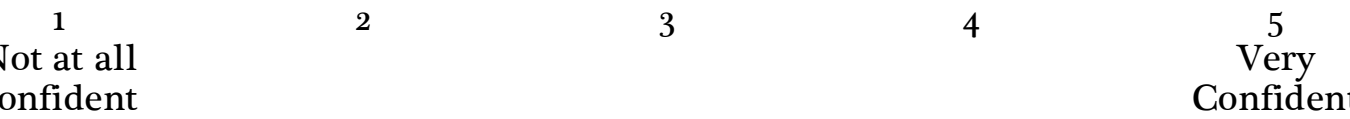

Now imagine that the first answer you gave is wrong. Please answer the question again with your second choice. Make sure you don't select the same answer as above.

What colour was Chad's hair?
a. Blonde
b. Brown
c. Black
d. Ginger
e. Grey

Please explain why you chose this answer as your second choice: 


\section{What colour was the kitchen bench?}
a. Green
b. Blue
c. Grey
d. Yellow
e. Orange

How confident are you that your answer is correct?

1

Not at all confident
2
3
4 Very Confident

Now imagine that the first answer you gave is wrong. Please answer the question again with your second choice. Make sure you don't select the same answer as above.

1a. What colour was the kitchen bench?
a. Green
b. Blue
c. Grey
d. Yellow
e. Orange

Please explain why you selected this answer as your second choice:

2. What colour cup did the person who opened the front door have?
a. Yellow
b. White
c. Green
d. Blue
e. Pink

How confident are you that your answer is correct?
Not at all
2
3
4 5 confident Confident

Now imagine that the first answer you gave is wrong. Please answer the question again with your second choice. Make sure you don't select the same answer as above.

2a. What colour cup did the person who opened the front door have?
a. Yellow
b. White
c. Green
d. Blue
e. Pink

Please explain why you selected this answer as your second choice: 
3. What colour balloons were hanging over the bathroom door?
a. Blue
b. White
c. Red
d. Yellow
e. Green

How confident are you that your answer is correct?

Not at all

2

3

4 5 confident Confident

Now imagine that the first answer you gave is wrong. Please answer the question again with your second choice. Make sure you don't select the same answer as above.

3a. What colour balloons were hanging over the bathroom door?
a. Blue
b. White
c. Red
d. Yellow
e. Green

Please explain why you selected this answer as your second choice:

\section{What number was the house the party was at?}
a. $2 \mathrm{~A}$
b. $5 \mathrm{~A}$
c. $12 \mathrm{~A}$
d. $22 \mathrm{~A}$
e. $122 \mathrm{~A}$

How confident are you that your answer is correct?

$\begin{array}{llccc}1 & 2 & 3 & 4 & 5 \\ \begin{array}{l}\text { Not at all } \\ \text { confident }\end{array} & & & & \begin{array}{c}\text { Very } \\ \text { Confident }\end{array}\end{array}$

Now imagine that the first answer you gave is wrong. Please answer the question again with your second choice. Make sure you don't select the same answer as above.

4a. What number was the house the party was at?
a. $2 \mathrm{~A}$
b. $5 \mathrm{~A}$
c. $12 \mathrm{~A}$
d. $22 \mathrm{~A}$
e. $122 \mathrm{~A}$

Please explain why you selected this answer as your second choice: 


\section{What did Chad add to the drink he made for the woman?}
a. A Sugar cube
b. A pinch of Salt
c. A Squeeze of lemon
d. A vial of liquid
e. A cherry

How confident are you that your answer is correct?

$\begin{array}{llccc}1 & 2 & 3 & 4 & 5 \\ \begin{array}{l}\text { Not at all } \\ \text { confident }\end{array} & & & & \begin{array}{c}\text { Very } \\ \text { Confident }\end{array}\end{array}$

Now imagine that the first answer you gave is wrong. Please answer the question again with your second choice. Make sure you don't select the same answer as above.

5a. What did Chad add to the drink he made for the woman?
a. A Sugar cube
b. A pinch of
c. A Squeeze of
d. A vial of liquid
e. A cherry

Please explain why you selected this answer as your second choice:

\section{What decoration was hanging over the doorway to the lounge?}
a. Tinsel
b. Streamers
c. Birthday Banner
d. Farewell
Banner
e. Congratulations

Banner Banner

How confident are you that your answer is correct?

1

Not at all

confident
2

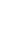

3
4
5 Very Confident

Now imagine that the first answer you gave is wrong. Please answer the question again with your second choice. Make sure you don't select the same answer as above.

6a. What decoration was hanging over the doorway to the lounge?
a. Tinsel
b. Streamers
c. Birthday
Banner
d. Farewell
e. Congratulations Banner Banner

Please explain why you selected this answer as your second choice: 
7. Where did Chad leave his bag when he got to the party?
a. Kitchen
b. Hallway
c. Bathroom
d. Bedroom
e. Lounge

How confident are you that your answer is correct?

$\begin{array}{llccc}1 & 2 & 3 & 4 & 5 \\ \begin{array}{l}\text { Not at all } \\ \text { confident }\end{array} & & & & \text { Very } \\ \text { Confident }\end{array}$

Now imagine that the first answer you gave is wrong. Please answer the question again with your second choice. Make sure you don't select the same answer as above.

7a. Where did Chad leave his bag when he got to the party?
a. Kitchen
b. Hallway
c. Bathroom
d. Bedroom
e. Lounge

Please explain why you selected this answer as your second choice:

\section{How did Chad get into the house?}
a. He walked straight in
b. He used his key
c. He made a phone call
d. He rang the doorbell
e. He knocked on the door

How confident are you that your answer is correct?

1

Not at all

confident
2

3
4 $\stackrel{5}{\text { Ver }}$ Confident

Now imagine that the first answer you gave is wrong. Please answer the question again with your second choice. Make sure you don't select the same answer as above.

\section{8a. How did Chad get into the house?}
a. He walked
b. He used his
c. He made a straight in key phone call
d. He rang the
e. He knocked on doorbell the door

Please explain why you selected this answer as your second choice: 
9. Where did Chad put the wallet he found on the bench?
a. In the
b. Behind the
c. Back where
d. In his back
e. In his bag cupboard microwave he found it pocket

How confident are you that your answer is correct?

1

Not at all confident
3
2
4
Very

Confident

Now imagine that the first answer you gave is wrong. Please answer the question again with your second choice. Make sure you don't select the same answer as above.

9a. Where did Chad put the wallet he found on the bench?
a. In the
b. Behind the
microwave
c. Back where he found it
d. In his back pocket
e. In his bag cupboard

Please explain why you selected this answer as your second choice:

\section{What did Chad sort through at the small table in the lounge?}
a. Cutlery
b. CDs
c. Bills
d. Photos
e. Tapes

How confident are you that your answer is correct?

\begin{tabular}{|c|c|c|c|}
\hline $\begin{array}{c}1 \\
\text { nf at all } \\
\text { nfident }\end{array}$ & 2 & 3 & 4 \\
\hline
\end{tabular}

Now imagine that the first answer you gave is wrong. Please answer the question again with your second choice. Make sure you don't select the same answer as above.

1oa. What did Ghad sort through at the small table in the lounge?
a. Cutlery
b. CDs
c. Bills
d. Photos
e. Tapes

Please explain why you selected this answer as your second choice: 
11. Where did Chad go when he was trying to find the toilet?
a. Conservatory
b. Bedroom
c. Linen
cupboard
d. Laundry
e. Wardrobe

How confident are you that your answer is correct?

1

Not at all

confident
2

3
4 5 Very Confident

Now imagine that the first answer you gave is wrong. Please answer the question again with your second choice. Make sure you don't select the same answer as above.

11a. Where did Ghad go when he was trying to find the toilet?
a. Conservatory
b. Bedroom
c. Linen
cupboard
d. Laundry
e. Wardrobe

Please explain why you selected this answer as your second choice:

12. What drink did Chad take out of his brown paper bag?
a. Beer
b. Wine
c. Rum
d. Vodka

e. Orange juice

How confident are you that your answer is correct?

1

2

3

4

5

Not at all

confident

Confident

Now imagine that the first answer you gave is wrong. Please answer the question again with your second choice. Make sure you don't select the same answer as above.

12a. What drink did Chad take out of his brown paper bag?
a. Beer
b. Wine
c. Rum
d. Vodka
e. Orange juice

Please explain why you selected this answer as your second choice: 


\section{Appendix H: Acuity Manipulation check}

(Experiment 5 and 6)

\section{Please circle your answers to the following questions based on your impressions while you were wearing the acuity glasses.}

How would you rate your vision while you watched the movie through the acuity glasses?

1

Extremely

degraded
2

Slightly degraded
4

5

As clear as normal vision

How would you rate the sharpness of the movie through the acuity glasses?

1

Very Fuzzy
2

3
3

45 Very Clear

How would you rate the brightness of the movie through the acuity glasses?

1

Very Dull
2

3

4

5

How would you rate the contrast of the movie through the acuity glasses?

1

Very Dull
2

3
4

5

How would you rate the vibrancy of the colours in the movie through the acuity glasses?

1

Very Dull
2

3
4

5

Very Bright

Did you notice any of your other senses feeling more switched on that usual while the acuity glasses degraded your vision?

1

No different to normal
3

4

5

Extremely switched on 
Please answer the following questions without looking on the front of your booklet or talking to your partner.

What strength of acuity glasses did you wear?

$35 \%$

$55 \%$

$75 \%$

$95 \%$

What strength of acuity glasses did your partner wear?

$35 \%$

$55 \%$

$75 \%$

95\% 


\section{Appendix I: Credibility manipulation check}

(Experiment 6)

\section{Please answer the following questions.}

Compared to your partner, how well do you think you could see the movie?

\begin{tabular}{ccccccc}
-3 & -2 & -1 & 0 & 1 & 2 & 3 \\
$\begin{array}{c}\text { His/her } \\
\text { vision was } \\
\text { better }\end{array}$ & & & $\begin{array}{c}\text { About the } \\
\text { same }\end{array}$ & & & $\begin{array}{c}\text { My vision } \\
\text { was better }\end{array}$ \\
\hline
\end{tabular}

Compared to your partner, how well do you think you could make out the details in the movie?
$-3$
$-2$
$-1$
O
1
2
3

He/she was better

About the same

Compared to your partner, how credible is your memory of the movie?

$-3$

$-2$

His/her

report is

more

credible
$-1$

O

About the

same
1

2

3

My report is more credible

Compared to your partner, how reliable is your memory of the movie?

$$
-3
$$

His/her report is more reliable
$-1 \quad 0$

About the same
3

My report is more reliable 


\section{Appendix J: Independent vs. Dyadic Analyses}

In Experiments $2 \mathrm{~A}, 2 \mathrm{~B}, 4,5$, and 6, people participated in pairs so that each member could expose the other to some misleading suggestions in the conversation. Put simply, each person acted as a vehicle to convey misinformation, and as such improved on the ecological validity of earlier misinformation effect research. Yet, because the people took part in pairs, it is possible that their responses were not independent, thus violating the statistical assumptions of the analyses.

If the pairs' responses were interdependent, but they were treated independently, it might underestimate or overestimate the effects found. Specifically, if the interdependence led pairs to behave in the same way, but they were treated independently, the analysis would underestimate the variance, and overestimate any effects found. Alternatively, if the interdependence led pairs to behave in different ways, but they were treated independently, it could overestimate the variance, and thus underestimate any effects found. An alternative approach is to use a dyadic or multilevel analysis, which would take into account any variation within pairs (Griffin \& Gonzalez, 1995; Kashy \& Kenny, 200o; Kenny, 1996; Kenny, Kashy \& Cook, 2006; Kraemer \& Jacklin, 1979).

To determine whether a dyadic analysis was necessary, I first examined the data from Experiments $2 \mathrm{~A}$ and $2 \mathrm{~B}$ to see whether there was any evidence of interdependence within the pairs' responses. I followed Kashy and Kenny's (2000) formulas, and calculated the intraclass correlations between subjects. Because the intraclass correlation is a low power test, Kashy and Kenny recommend using a liberal alpha level of .20 to determine whether there is any relationship between subjects' responses. In Experiment $2 \mathrm{~A}$ - even using the liberal alpha of .20 - there was no evidence of interdependence in subjects responses to either discussed details, $r_{I}=0.19, F(18,19)=1.48, p=.40(2$ tailed $)$, or nondiscussed 
details, $r_{I}=-0.08, F(20,19)=1.18, p=.72(2$ tailed $)$. Similarly, in Experiment $2 \mathrm{~B}$, there was no evidence of interdependence for subjects responses to discussed details, $r_{I}=0.27, F(19,20)=1.72, p=.24(2$ tailed $)$ or nondiscussed details, $r_{I}=-0.13$, $F(20,19)=1.29, p=0.58$ ( 2 tailed $)$. In short, there was no evidence of interdependence between subject-pairs' responses. These results suggest that it is acceptable to use the individual as the unit of analysis.

Although a significant correlation would provide evidence of a relationship, lack of a correlation does not necessarily rule out the possibility that there is some relationship. I next examined whether treating subjects as independent affected the results in any way. One way to examine whether interdependence has any impact on the results is to exclude a random member of every pair and conduct the same analyses (Kraemer \& Jacklin, 1979). If the same patterns of results hold true, it suggests that any interdependence does not affect the results. I repeated the major analyses from Experiments $2 \mathrm{~A}$ and ${ }_{2} \mathrm{~B}$, with one member from each pair randomly excluded from the analyses. These results are displayed on the figures below, alongside the results from Experiments $2 \mathrm{~A}$ and $2 \mathrm{~B}$. As the figures illustrate, I found exactly the same pattern of results, suggesting that any interdependence does not cause a problem for these data.

Taken together, these results suggest that it is acceptable to treat each subject as an independent data point. There was no evidence of a relationship between subjects' responses (within pairs), and no evidence that treating subjects as independent affected the results in any way. As such, I treated each subject as an independent data point throughout the thesis. 


\section{Experiment 2A}
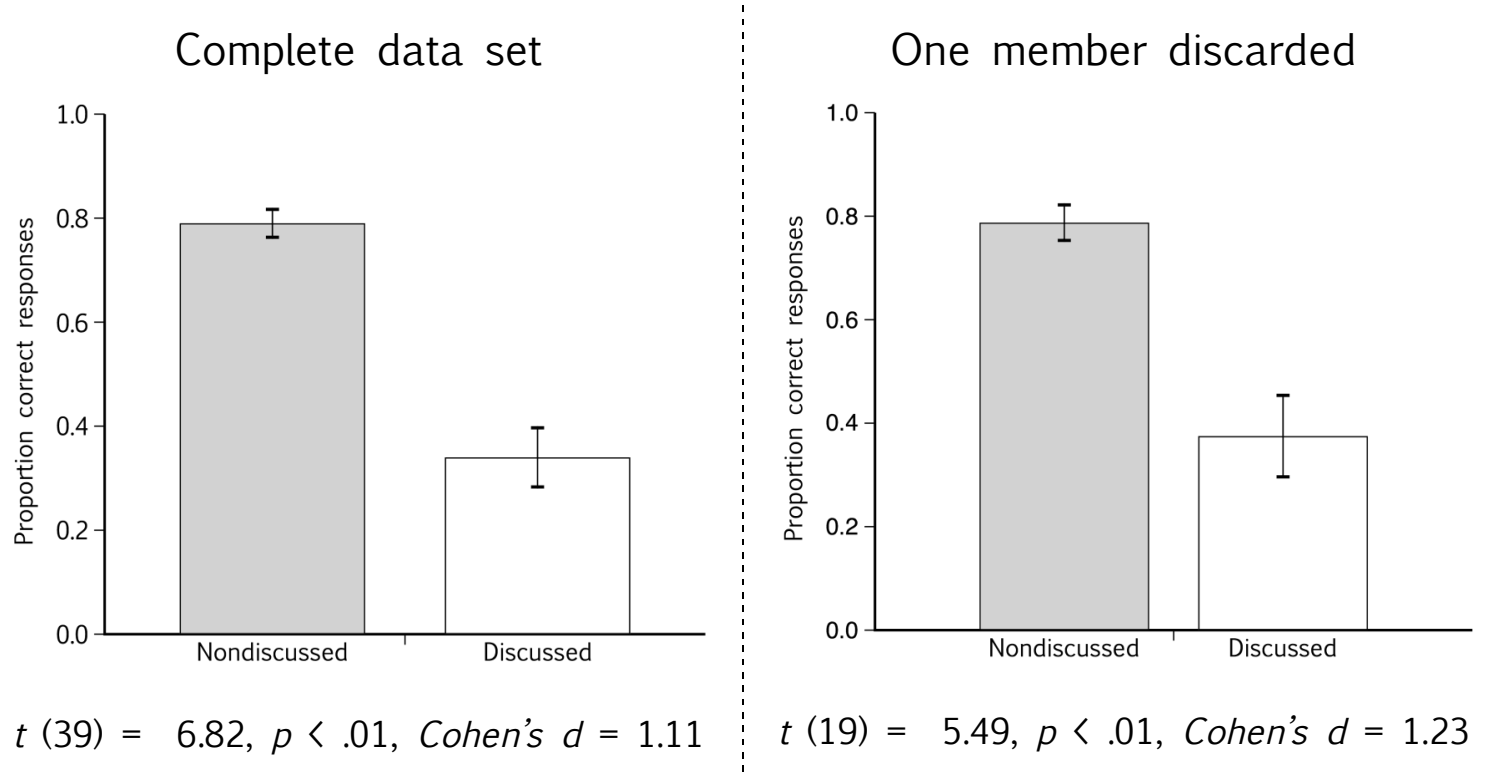

\section{Experiment 2B}

Complete data set

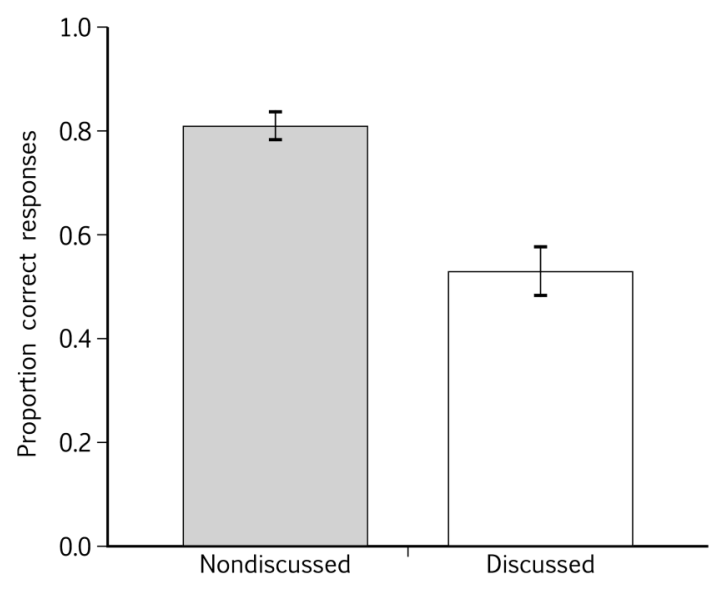

$t(39)=5.64, p<.01$, Cohen's $d=0.88$
One member discarded

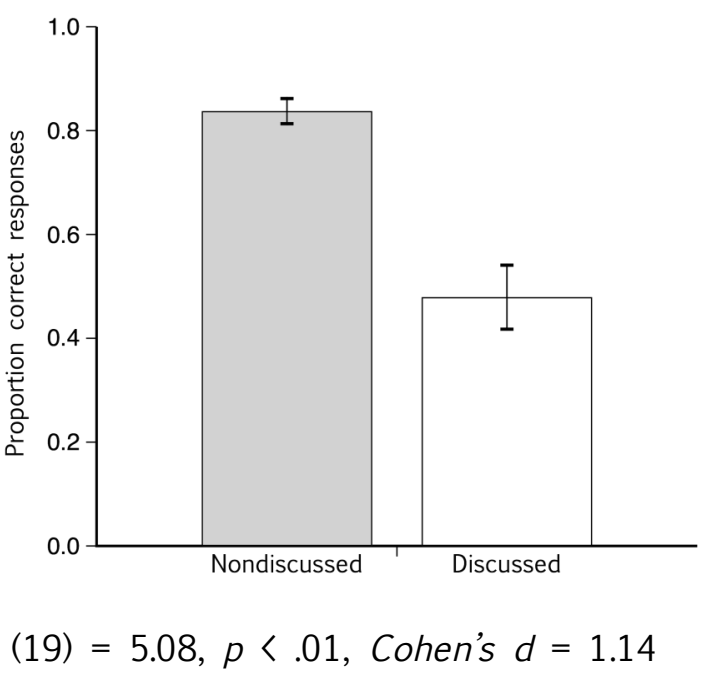




\section{Author Index}

Aberson, C., 90, 183

Allan, K., 14, 17, 187

Alper, A., 15, 183

Andersson, J., 170, 183

Asch, S. E., 9, 56, 151, 168, 175, 177, 183, 184

Ask, K., 13, 187

Assefi, S. L., 116, 132, 162, 183

Barclay, C. R., 1, 2, 183

Bartlett, F. C., 1, 2, 3, 184, 186

Basden, B. H., 6, 170, 184

Basden, D. R., 6, 170

Bazerman, M. H., 25, 197

Belli, R. F., 4, 184

Bellinger, K. D., 1, 2, 170, 198

Bergman, E. T., 17, 195

Berkowitz, S. R., 79, 191

Bernstein, D. M., 79, 191

Betz, A. L.,, 6, 184

Blair, C., 170, 198

Bless, H., 6, 198

Bond, R., 10, 25, 184

Bottrill, K. V., 6, 188

Bradfield, A. L., 18o, 184

Bradshaw, J. M., 6, 29, 53, 55, 78, 98, 113, 116, 132, 160, 185

Brett, J. M., 25, 197

Brewer, N., 180, 184

Brown, N., 1

Bryner, S., 184

Buckhout, R., 15, 183

Burke, A., 18o, 184

Burns, H. J., 4, 192

Bush, J. G., 49, 189

Carrere, S., 99, 187

Castle, S., 13, 184

Ceci, S. J., 6, 15, 19, 116, 16o, 184, 195

Chapman, L., 98, 194

Chern, S., 15, 183

Clifasefi, S. L., 116, 132, 162, 185, 194

Clifford, B. R., 15, 188

Coan, J., 99, 187

Cockman, D. W., 29, 174, 188

Conway, M., 1, 189, 195

Cook, G. I., 29, 174, 185, 190, 225

Cook, W. L., 225

Cowell, A., 13, 185

Craik, F. I. M., 165, 185, 193

Cuc, A., 2, 3, 16, 18, 185

De Leonardis, D. M., 193

DeCooke, P. A., 1, 183

Deutsch, M., 10, 6o, 72, 109, 168, 170, 176, 185

Dodd, D. H., 6, 29, 53, 55, 78, 98, 113, 116, 132, 16o, 185

Duensing, S., 2, 192

Dunning, D., 161, 186, 191

Echterhoff, G., 3, 6, 29, 53, 55, 78, 98, 99, 113, $116,132,160,176,186$
Edwards, D., 1, 2, 3, 186

Ehrlinger, J., 161, 186

Ellsworth, P. C., 5, 6, 29, 49, 54, 78, 96, 113, 195

Engle, R. W., 116, 194

Erber, R., 12, 198

Faries, J. M., 2, 189

Finlay, F., 170, 186

Fivush, R., 1, 2, 186

Foley, M. A., 27, 190

Foster-Fishman, P. G., 172, 191

Fraidin, S., 12, 96, 114, 188

Franz, T. M., 172, 191

French, L., 2, 3, 16, 186, 200, 201

Gabbert, F., 14, 17, 18, 19, 30, 62, 63, 64, 110, 111, $113,117,118,125,130,131,132,147,148,158$, 16o, 162, 178, 187, 189, 191

Garry, M., iv, 2, 4, 5, 116, 132, 162, 183, 185, 186, 194, 196, 197

Garven, S., 17, 195

Gerard, H. B., 10, 6o, 72, 109, 168, 170, 176, 185

Gilbert, D. T., 170, 187

Giuliano, T., 11, 198

Gonzalez, R., 187, 225

Gottman, J. M., 99, 112, 187

Granhag, P. A., 6, 13, 187, 188

Griffin, D., 187, 225

Groll, S., 3, 186

Gruenfeld, D. H., 7, 199

Gulgoz, S., 2, 189

Haden, C., 1, 186

Halbwachs, M., 2, 187

Hall, D., 151, 183, 197

Hardin, C. D., 1, 99, 112, 187

Harkins, S., 170, 191

Harper, D. N., 116, 185, 187, 194

Harsh, N., 194

Harwood, R., 15, 183

Hashtroudi, S., iii, 26, 190

Hayne, H., 1, 192

Healey, S., 113, 189

Hertel, P. T., 11, 198

Hewitt, P. G., 20, 188

Hicks, J. L., 29, 174, 185, 188

Higgins, E. T., 1, 3, 99, 112, 186, 187, 195

Highhouse, S., 6, 188

Hirokawa, K., 25, 188

Hirst, W., 2, 6, 29, 53, 55, 78, 98, 113, 116, 132, 16o, 176, 185, 186

Hitch, G. J., 170, 186, 193

Hoffman, H. G., 6, 188

Hollin, C. R., 15, 188

Hollingshead, A. B., 11, 12, 96, 99, 112, 114, 173, 188

Holtgraves, T., 29, 174, 189

Hood, L., 14, 189

Hope, L., 113, 189

Horn, M., 25, 196

Huebsch, P. D., 170, 198 
Hussy, W., 6, 186

Hyman, I. E., Jr., 2, 189

Ikier, S., 2, 3, 189

Itoh, Y., 25, 189

Itsukushima, Y., 6, 8, 30, 189

Jacklin, C. N., 19o, 225, 226

Johnson, K., 161

Johnson, M. K., iii, 25, 26, 27, 28, 29, 49, 54, 96, $161,163,164,165,174,186,189,190,191,193$

Johnson, T., 25

Justice, C., 14

Kaasa, S. O., 79, 191

Kanematsu, H., 19, 22, 23, 24, 34, 35, 190

Kashy, D. A., 190, 225

Kassin, S. M., 17, 190

Kawaguchi, J., 25, 189

Kemp, R. I., 15, 17, 18, 30, 98, 162

Kemp, S., 2

Kenny, D. A., 190, 225

Keys, C. B., 172, 191

Kiechel, K. L., 17, 190

Kitabayashi, M., 25, 194

Klumpp, A., 170, 198

Kniveton, B. H., 11, 196

Kowner, R., 25, 190

Kraemer, H. C., 19o, 225, 226

Kronlund, A., 166, 190

Kruger, J., 161, 186, 191

Krull, D. S., 170, 187

Kuntay, A. C., 2, 189

Kwong, T., 6, 188

Lalwani, A. K., 25, 191

Laney, C., 79, 80, 191

Larson, J. R., Jr., 172, 191

Latané, B., 170, 191

Ledivow, B., 2, 192

Lenton, E., 113, 189

Lindsay, D. S., iii, 4, 26, 27, 28, 29, 53, 54, 62, 63 , $65,75,96,110,130,132,159,163,164,165,167$, $174,178,190,191$

Lockhart, R. S., 165, 185

Loftus, E. F., 2, 4, 5, 6, 53, 79, 90, 104, 151, 188, 189, 191, 192, 197

Loftus, G., 90, 192

Luus, C. A. E., 6, 180, 192

MacDonald, S., 1, 192

Manier, D., 2, 185

Marsh, E. J., 1, 3, 29, 185, 192

Marsh, R. L., 29

Maruyama, M., 6, 189

Mather, M., 29, 174, 193

Mathews, S. A., 17, 199

Matsuno, E., 25, 188

McCloskey, M., 4, 5, 53, 193

Meade, M. L., 1, 5, 17, 165, 179, 193, 195

Memon, A., 14, 17, 18, 187, 191, 193

Merritt, D., 196

Meudell, P. R., 170, 186, 193

Middleton, D., 1, 2, 3, 186
Miller, D. G., 4, 192

Milton, H., 15, 197

Mitchell, K. J., 26, 27, 28, 49, 165, 189, 193

Moore, D. A., 25, 197

Mori, H., 19, 25, 34

Mori, K., ii, 19, 22, 23, 25, 34, 188, 190, 193, 194

Morris, E. K., 79

Morris, S., 15

Neisser, U., 2, 183, 194

Nelson, K., 1, 194

Nishi, M., 6, 189

Okumura, T., 25, 197

Ost, J., 113, 189

Ostrom, T. M., 6, 184

Ozuru, Y., 2, 185

Palmer, J. C., 4, 192

Parker, S., 4, 116, 132, 162, 194, 196

Paterson, H. M., 15, 17, 18, 30, 98, 162, 194

Pelham, B. W., 170, 187

Pezdek, K., 61, 197

Pickel, K. L., 180, 194

Principe, G. F., 15, 19, 195

Rackstraw, P., 6, 198

Raye, C. L., 27, 190

Raymond, P., 12, 198

Rebelius, A., 13, 187

Reese, E., 1, 186, 195

Reysen, M. B., 6, 184, 195

Roediger, H. L., III., 1, 5, 17, 165, 179, 191, 193, 195

Ronnberg, J., 170, 183

Ross, D. F., 6

Ross, M., 1, 2, 3

Rubin, D., 2, 183, 186, 189, 195

Saito, S., 170, 196

Sanders, G. S., 15, 198

Schneider, D. M., 17, 195

See, S., 6, 188

Self, G., 14

Sharman, S. J., 5, 116, 185, 197

Shavitt, S., 25, 191

Shaw, J. S., 17, 195

Sheen, M., 2, 3, 195

Skagerberg, E. M., 14, 17, 117, 118, 125, 132, 147, 160, 195, 199

Skowronski, J. J., 6, 184

Slomovitz, M., 15, 183

Smith, P. B., 10, 25

Smith, V. L., 5, 6, 29, 49, 54, 78, 96, 113

Socall, D., 29, 189

Srull, T. K., 29, 189

Stasser, G., 170, 172, 196

Stephenson, G. M., 11, 173, 196

Stewart, D. D., 172, 196

Strack, F., 6, 198

Suengas, A. G., 27, 190

Sun, T., 25, 196

Sutherland, R., 2, 116, 185, 186

Swanson, C., 99, 187 
Takahashi, M., 6, 170, 189, 196

Takarangi, M. K. T., viii, 4, 5, 31, 34, 35, 36, 37, $4 \mathrm{O}, 42,43,44,48,53,66,196$

Tekcan, A. I., 2, 189

Tenbrunsel, A. E., 25, 197

Thomas, J., 14, 170, 184, 196, 197

Thomas, R. L., III., 14, 170, 184, 196, 197

Thompson, B., 90, 196, 197

Toglia, M. P., 6, 184

Tousignant, J. P., 151, 197

Tulving, E., 165, 185, 193

Tversky, B., 1, 3, 192, 195

Uesiliana, K., 1, 192

Ukita, J., 25, 188

Umeda, S., 25, 189

Underwood, G., 15

Underwood, J., 61

Vornik, L. A., 5, 6, 7, 30, 53, 55, 98, 113, 116, 132, 16o, 197

Wade-Benzoni, K. A., 25, 197
Wagner, D., 6

Wagner, W., 11

Walther, E., 6, 198

Warnick, D. H., 15, 198

Watkins, M. J., 17, 195

Wegner, D. M., 11, 12, 13, 96, 99, 100, 112, 114, 173,198

Weldon, M. S., 1, 2, 170, 198

Wells, G. L., 6, 180, 184, 192

Werth, L., 6, 198

Whittlesea, B. W. A., 166, 190

Williams, K., 170, 191

Wiseman, R., 25, 190

Wittenbaum, G. M., 172, 196, 198

Wood, J. M., 17, 195

Wright, D. B., 14, 17, 18, 117, 118, 125, 132, 147, 16o, 170, 187, 191, 193, 195, 198, 199

Wyer, R. S., 7, 199

Yarmey, A. D., 15, 199

Zanni, G., 4, 192

Zaragoza, M., 4, 5, 53, 193 


\section{Subject Index}

acuity glasses, 37, 38, 119, 134, 136, 139, 176, 214, 222, 223

alternate movie, vi, ix, $\mathrm{x}, 32,76,77,78,79,80$, $81,82,83,84,85,86,87,91,92,93,94,95$

Anna Lindh (murder of), 13, 187

asparagus, 80

audience, 3

belief, 169

capitulation, 116, 166, 168, 169, 175, 176

collaborative inhibition, 170, 171, 172, 196, 198

collectivist, 25

confederate, $17,18,175$

confidence, ix, $x, 7,8,30,46,47,48,58,59,69$, $70,71,89,90,91,92,105,106,107,125,126$, $127,152,153,154,180,183,184,192,209$

conformity, 9, 18, 22, 25, 28, 113, 117, 177, 184, $187,189,194,195,199$

consistency, 13, 57, 68, 72, 83, 100, 101, 105, 107, 109, 110, 111, 114, 123, 124, 131, 147, 148, 149, $156,159,163,166,173,178,180,181$

conversational rule, 18,59

corroboration, iii, 180

credibility, 7, 10, 27, 55, 96, 113, 115, 117, 118, 139, 141, 155, 16o, 161, 164, 175, 180, 181, 224

depth perception, 20

expectancy, viii, $\mathrm{x}, 116,120,123,125,126,127$, 130, 141, 144, 151, 153, 154

expertise, $5,11,12,49,113,114,115,171,172,173$, 181, 188, 195

exposure (to misinformation), viii, 3, 4, 5, 7, $16,17,18,26,31,35,53,54,56,59,60,61,62$, $63,64,65,68,69,70,72,73,74,75,102,103$, 104, 105, 107, 108, 110, 111, 112, 122, 124, 125, $128,129,130,131,132,142,148,149,150,151$, $155,156,157,158,159,164,170,171,172,173$, 178,179

eyewitness, 2, 13, 14, 23, 98, 115, 180, 184, 188, 190, 191, 192, 193, 194, 195, 197, 198, 199

familiarity, 27, 28, 98, 114

groups (remembering in), iii, iv, 6, 9, 11, 15, $16,25,32,81,82,83,84,115,119,125,130,141$, $144,145,146,147,148,149,150,152,154,156$, $158,161,168,170,172,183,184,198$

implicit memory, 32, 85, 86

individualistic, 25

inferences, 11

informational influence, 10, 6o, 72, 168, 169, 185

intervention, 98

Jack, 27, 28, 165, 166, 172, 178

judgments (social), 6, 7, 8, 9, 10, 12, 27, 29, 30, $55,57,69,81,83,96,98,112,113,114,115,116$,
$117,118,124,125,147,151,173,174,176,181$, $183,184,185,195,199$

justice system, 2, 179, 180, 182

knowledge, 1, 2, 5, 7, 12, 13, 27, 29, 113, 114, 166, 188,196

mechanism, 5, 17, 152, 154, 161, 179, 182

misinformation effect, $4,5,6,26,28,31,34,49$, $65,66,85,96,100,104,116,123,144,146,183$, $185,194,196,197,199,225$

misinformation messenger, vi, 5, 6, 7, 8, 33, $54,61,96,98,112,113,115,116,117,118,119$, $120,123,125,126,127,129,132,141,143,144$, $146,147,148,149,150,151,152,153,154,155$, $156,157,158,160,164,165,174,176,181$

Mollie, 27, 28, 86, 165, 166, 172, 178

MORI technique, ii, v, vii, ix, 19, 22, 23, 24, 25, 30, 31, 32, 34, 35, 37, 39, 44, 48, 49, 65, 79, 8o, $81,85,86,87,90,92,95,135,136,163,177,194$

narrative description, $8,26,28,31,34,38,40$, $45,48,49,50,53,117,162,194$

natural discussion, 17,26

normative influence, 10, 6o, 72, 109, 168, 169, $170,172,175,185$

Oklahoma (bombing), 14, 193, 197

personality characteristics, $9,56,80,83,151$, 177,190

placebo, 116, 162

polarisation (of light), ix, 19, 20, 21, 22, 23, 24, $35,38,39,40,82$

qualitative characteristics, 27

Red Herring technique, vii, 79, 80, 81, 83, 191, 214

relationships, $\mathrm{x}, 1,2,9,10,11,12,25,32,97,98$, 99, 100, 101, 102, 104, 105, 106, 107, 110, 112, $113,164,173,175,176,177,181,186,187,188$, 189, 191, 198, 225, 226

reminisce, 4,186

retrieval strategies, 170,184

second-choice test, 32, 86, 89

shared reality, 99, 186

social appraisal, 54, 80, 174, 175, 176

social dynamics, $9,12,18,30,31,49,162,163$, $164,168,176,194$

social influence, iii, $7,8,10,30,185$

social judgments, $6,7,8,9,10,12,27,29,30$, $55,57,69,81,83,96,98,112,113,114,115,116$, $117,118,124,125,147,151,173,174,176,181$, $183,184,185,195,199$

social pressure, 9, 10, 13, 52, 168, 169, 170, 173, 183

sociolinguistic (cues), 7, 18

Source Monitoring Framework, iii, v, vii, 26, $27,28,29,30,32,54,78,96,97,98,115,163$, 
$164,165,166,167,169,174,175,176,177,190$, 191, 193

source of information, 29, 55, 96, 164, 176 speaking order, 59, 61, 62, 63, 64, 65, 73, 74, 75, $107,110,111,112,127,130,131,132,156,159$,

$160,178,179$
Transactive Memory Systems, iii, v, vii, 8, 10, $11,12,13,96,99,113,114,173,174,188,198$ trust, 1, 13, 32, 96, 98, 100, 114, 214 